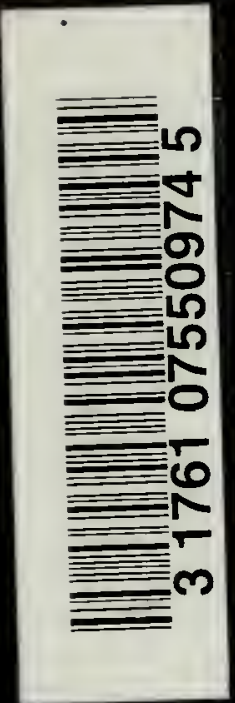

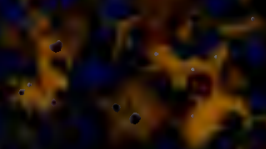

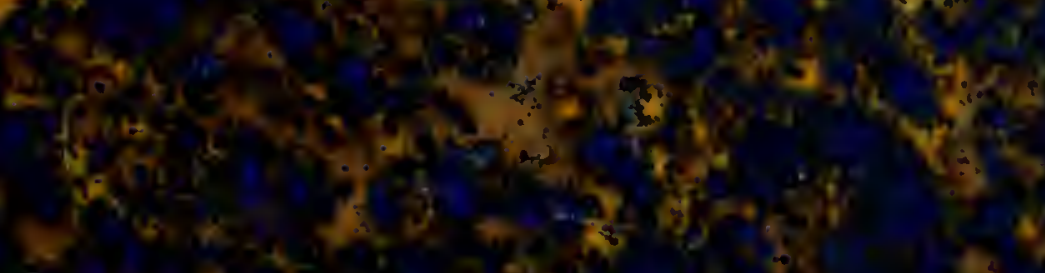

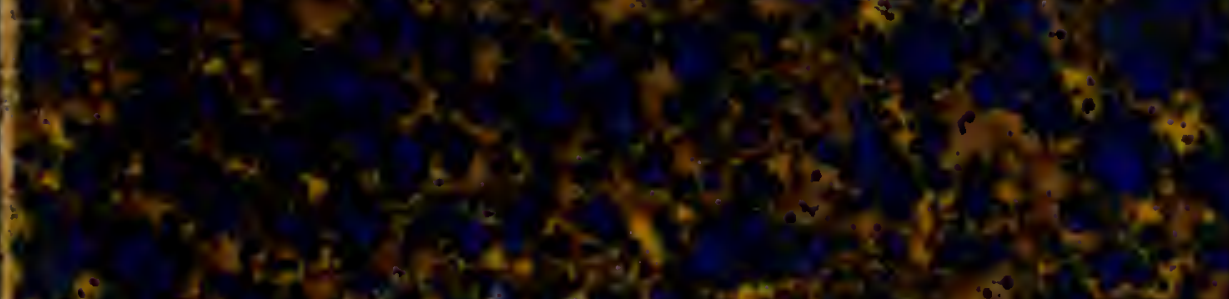

$\left(\begin{array}{c}0 \\ 6\end{array}\right.$

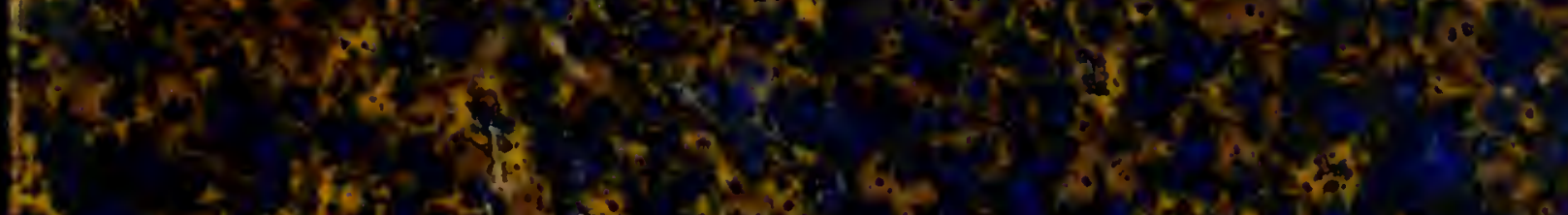

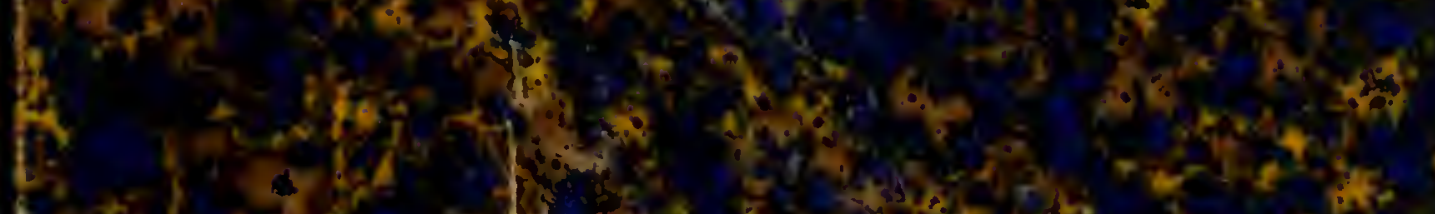

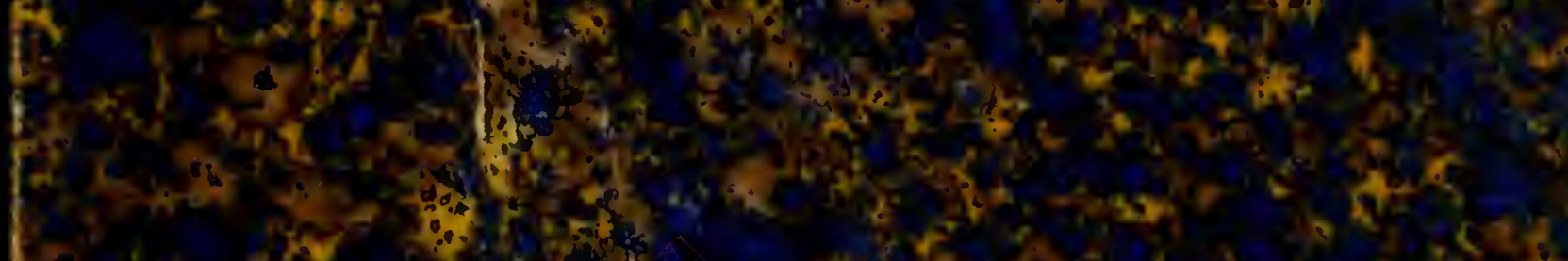

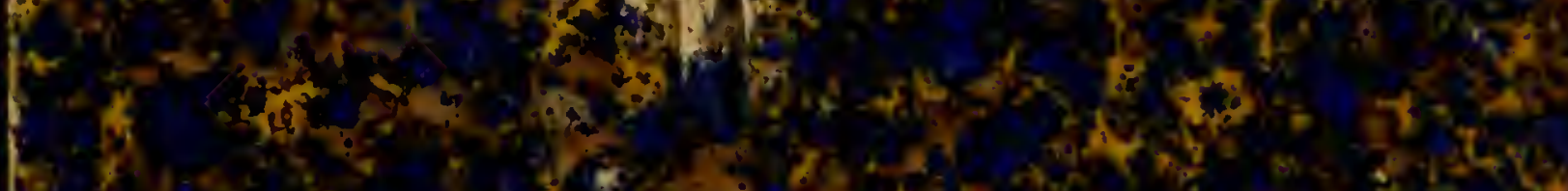

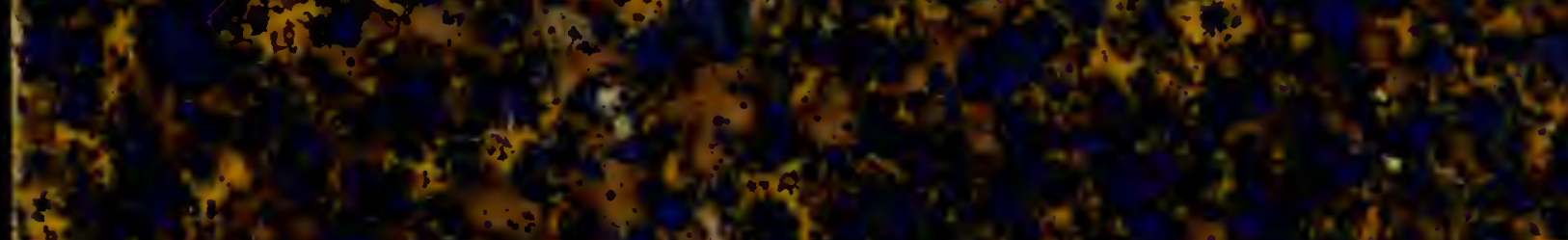

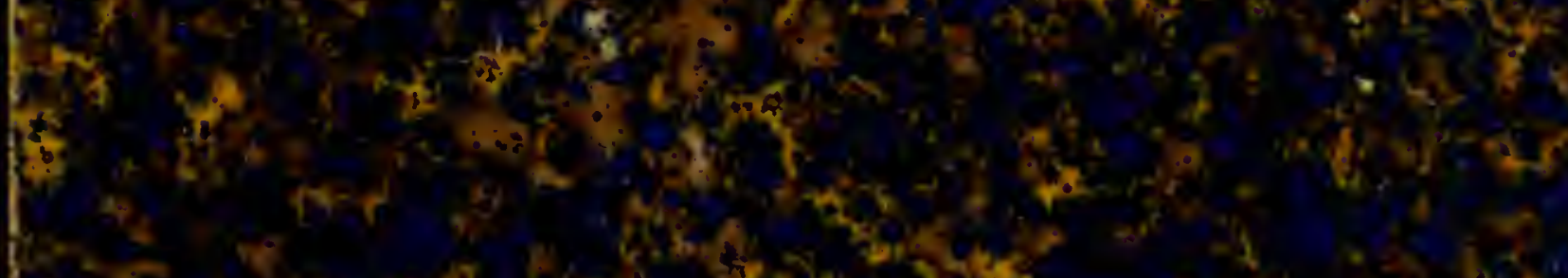

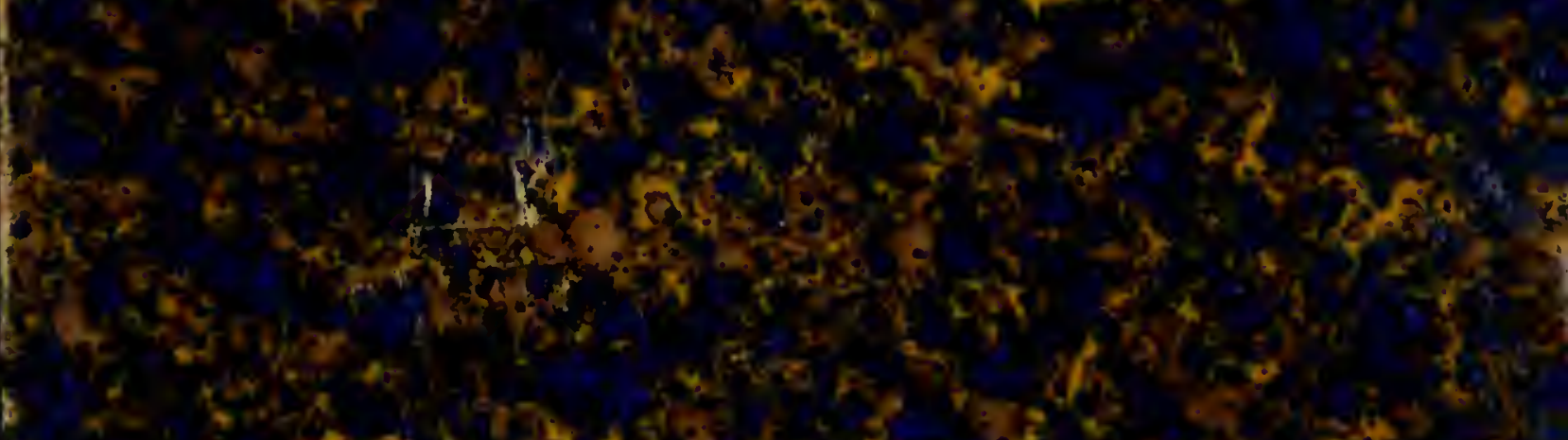

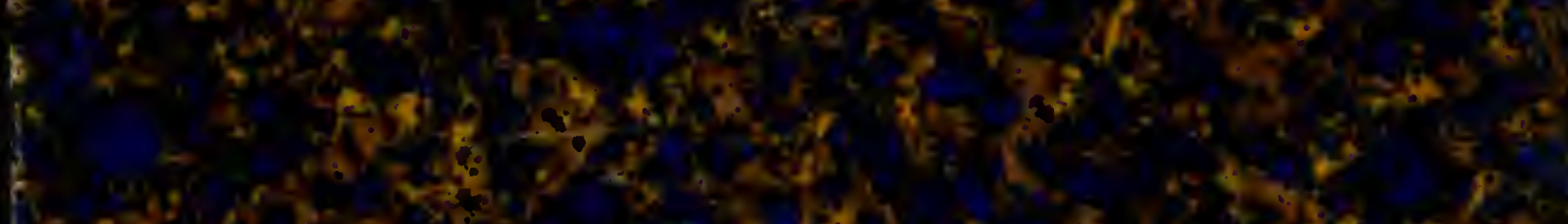
3.8. not

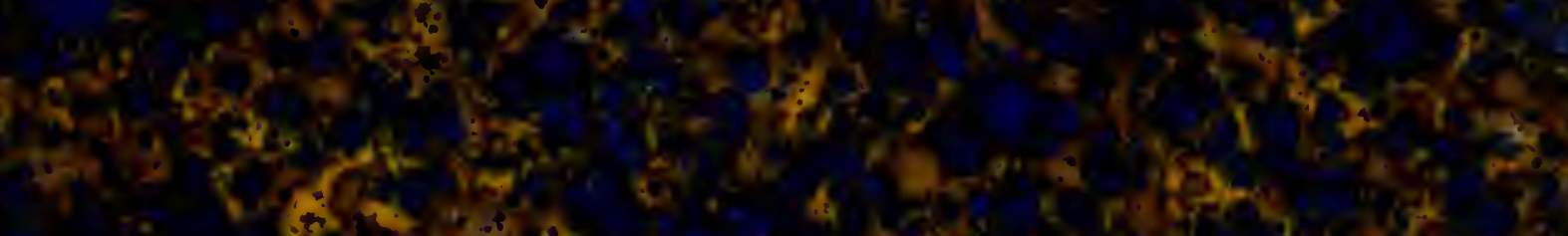

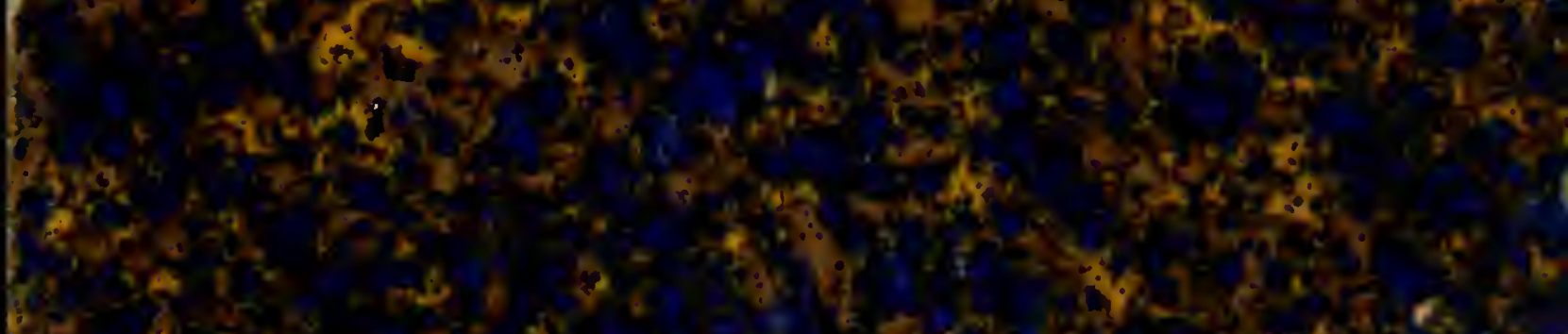




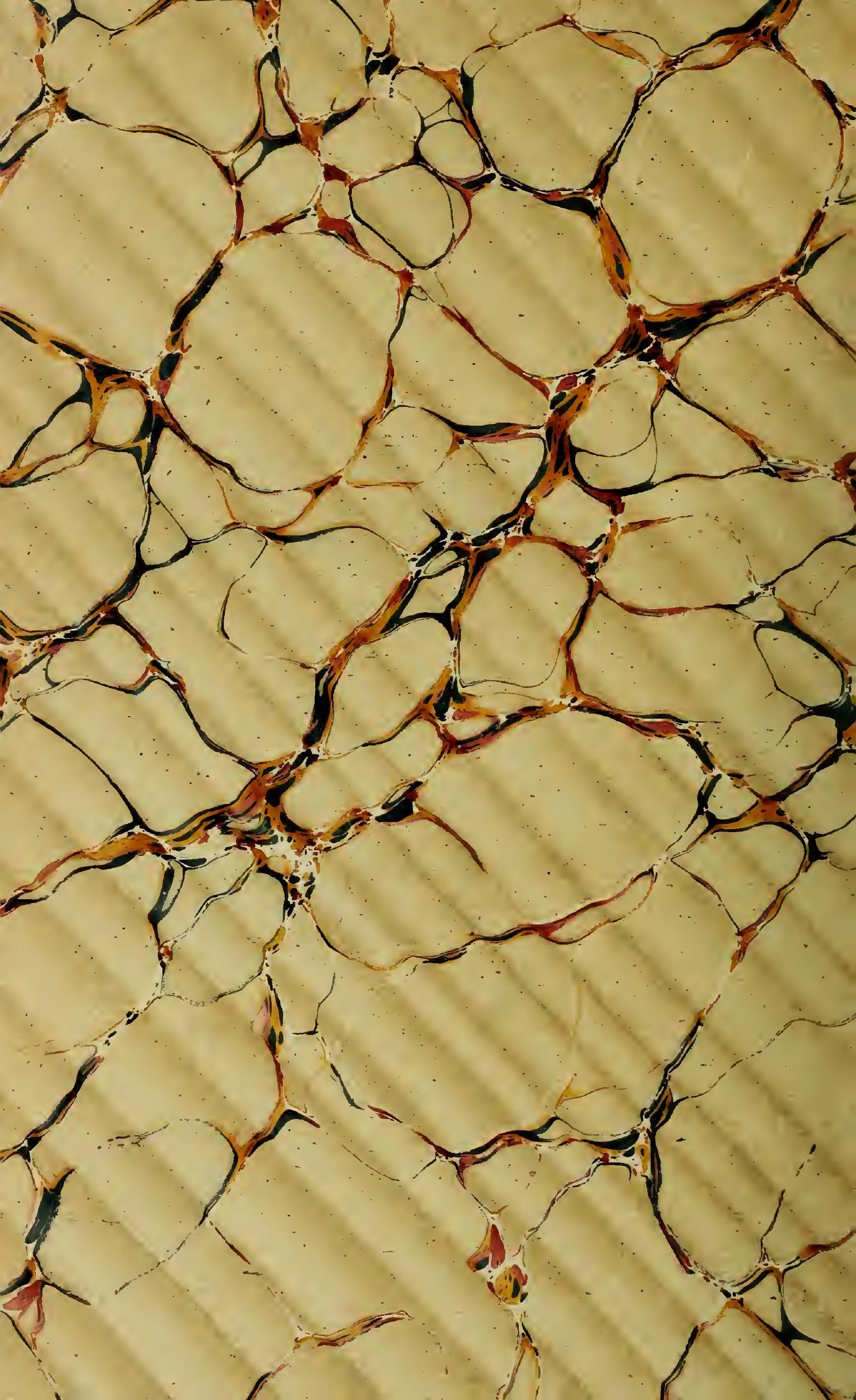




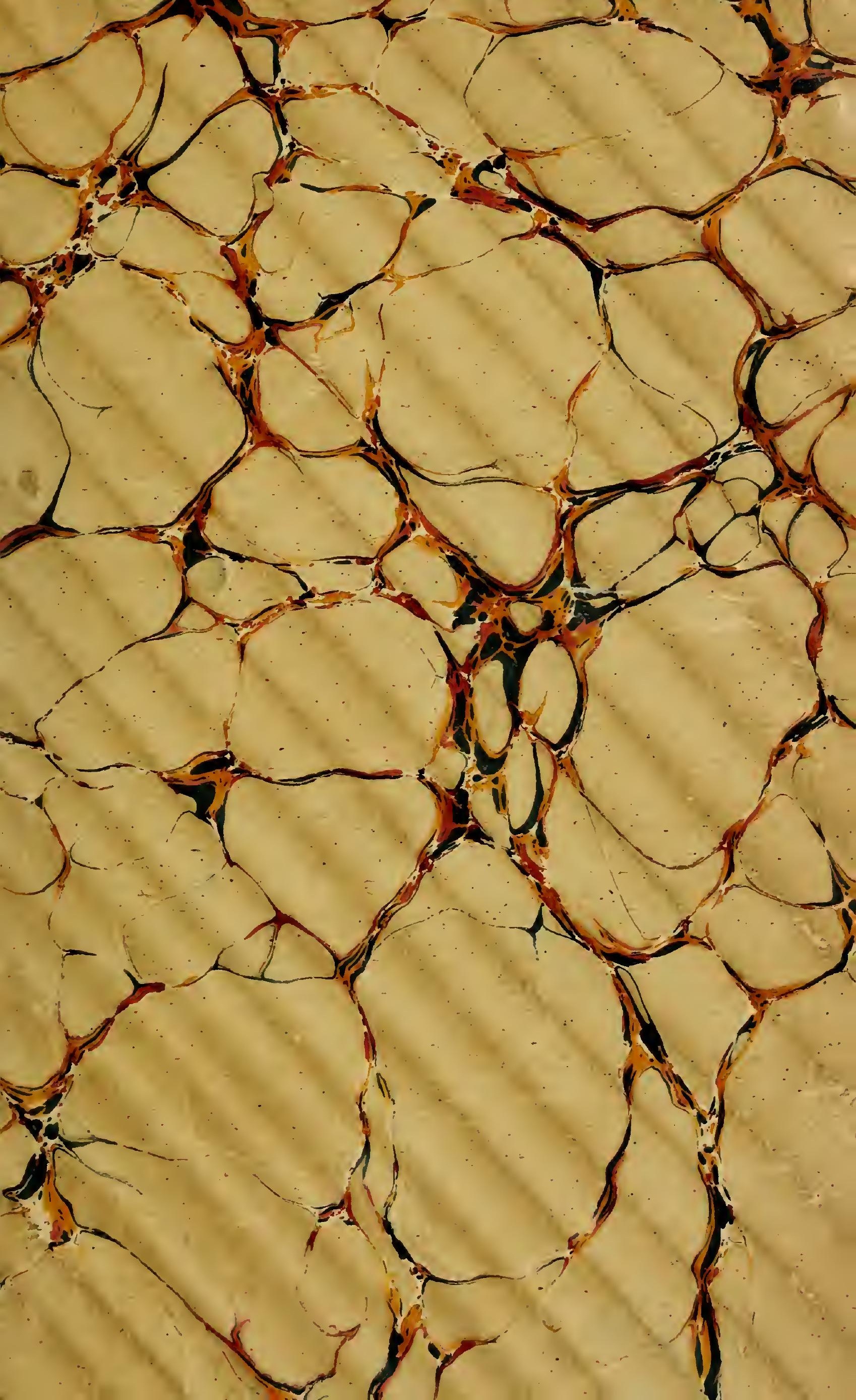







\section{TRAITÉ}

\section{D’ÉCONOMIE FORESTIËRE}

$$
\text { PAR }
$$

$$
\text { A. PUTON }
$$

\section{Membre du Conseil supérieur de lagriculture}

Dirceteur de l'Éeole nationale forestière

\section{LIBRARY \\ 2. \\ UNIVERSITY OF TORONTO}

\section{PARIS}

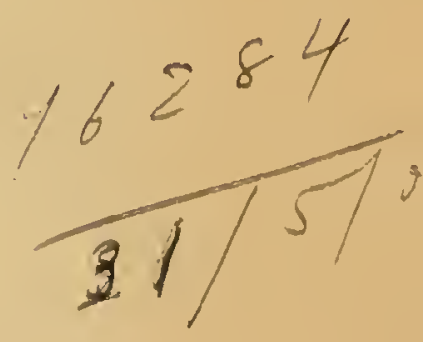

IIAOIIAI \& BIIIAED

Libraires de la Cour de Cassation

PLACE DAUPHINE, 27

1888 


\section{SD 393 P8}

Nancr. - Impr. Nancéienne, 1, rue de la Pépinière, - Dir. : Pierson. 


\section{INTRODUCTION}

La science forestière comporte plusieurs branches:

$1^{\circ}$ L'arboriculture forestière étudie les essences considérées individuellement, leurs exigences culturales et les soins à leur donner. L'étude de la taille, de l'élagage, de l'émondage, les questions relatives à la naturalisation et à l'acclimatation des espèces étrangères, les cultures en tétard et en émonde, sont de son ressort ainsi que celles des mûriers, des châtaigniers, des chênes lièg'es, des arbres d'ornements pour les parcs, les avenues, les routes, les squares et les boulevards des villes. La culture des pépinières de basses et de hautes tiges en forme l'annexe naturelle.

$2^{\circ}$ La sylviculture est l'étude du traitement des forêts, c'est l'application aux forêts des connaissances fournies sur la végzétation des essences sociables par les sciences naturelles, physiques et chimiques. Elle est une science de faits et d'observations plus que de raisonnement et de théorie. D'origine relativement récente, car elle était inconnue des anciens forestier's, elle a reçu un nom anjourd'hui consacré par l'usage en France et en Allemagne (Waldbau), mais assez mal choisi puisqu'on ne pratique dans les massifs forestiers ni labours, ni engrais, ni drainages, ni aucune des opérations du cultivateur. La forêt est un bien naturel; le propriétaire s'y applique à suivre 
les indications de la nature et à les développer sans les entraver jamais, règle de conduite fort simple, mais dont l'application est délicate et exige plus de science qu'on ne le croit généràlement. On y comprend, aussi, les règles de conduite destinées à défendre les forêts contre l'action des agents atmosphériques, les animaux nuisibles et les végétaux parasites, ce que les Allemands appellent protection des forêts.

Tel est le domaine de la sylviculture, restreint dans son champ d'action, mais varié et vaste par ses observations locales. Les reboisements artificiels par semis et par plantations, en forêts et en terrains dénulés, forment une partie importante de la sylviculture, notamment pour la restauration des montagnes dégradées.

$3^{\circ}$ La technologie forestière traite de l'application de la matière ligneuse aux différents usages de la vie: emploi du bois au chauffage, aux constructions civiles et navales, utilisations mécaniques et chimiques, qualités et défauts des bois, procédés d'abatag'e et de façonnagee, débits en marchandises de toutes sortes, cubage et estimation des bois abattus en vue de ces débits, usages commerciaux, etc.

Cet ensemble des apptications techniques du bois forme une branche importante de la science forestière, la plus ancienne et peut-être la seule connue des premiers forestiers. Elle s'est considérablement élargie par les emplois nouveaux de la matière ligneuse dans l'industrie moderne.

$4^{\circ}$ La dendrométrie cherche les moyens de déterminer les volumes absolus des arbres et des peuplements sur pied : instruments de mesurage pour les hauteurs et les diamètres, calculs des volumes et des rendements en vue d'évaluations rapides ou de recherches scientifiques. Cette partie tend à se développer et à gagner en précision par l'établissement des stations de recherches et d'expérimentation forestières. 
$5^{\circ}$ L'économie forestière s'occupe de la production de la richesse forestière : elle considère l'arbre comme un ouvrier fabricant de bois, le peuplement comme une association d'ouvriers unis pour une fabrication commune, la forêt comme une entreprise, une sorte d'usine destinée à produire la matière ligneuse. L’économie politique étudie la production, la circulation et la consommation de la richesse en général; l'économie forestière fait la même étude pour la richesse forestière : étude de l'arbre et du peuplement, de l'ouvrier isolé et des ouvriers associés, producteurs l'un et l'autre de volume et de valeur; lois de cette production et disposition de ces éléments dans la forêt. Celle-ci forme une entreprise, une véritable exploitation comme la ferme est une exploitation agricole: l'une produit le bois aux multiples usages, l'autre les denrées alimentaires, végétales et animales; l'une et l'autre ont leurs règles d'organisation et leurs buts de production. L'aménagement est un cas particulier de cette exploitation à bois, car c'est une combinaison, une disposition spéciale faite en vue d'une production annuelle.

$6^{\circ}$ L'estimation forestière comprend les règles relatives à l'évaluation des forêts en fonds et superficie, des torts et dommages qui peuvent leur être causés et des démembrements de leur propriété. C'est l'art délicat des expertises dans les expropriations, les rachats de servitudes et les cantonnements de droits d'usage. Cet art procède naturellement de l'économie forestière, c'est-à-dire de la connaissance exacte de la manière dont les exploitations forestières sont constituées.

Ces grandes divisions ne concernent que la science forestière proprement dite. Le propriétaire et l'administrateur de forêts doivent, en outre, faire appel aux applications des sciences juridiques, administratives, historiques et mathématiques : législation forestière, administration, histoire des forêts, arpentage, nivellement, con- 
struction de maisons, d'usines, de routes, correction des torrents, etc...

La science forestière ne possède pas encore de traité général ; moins heureuse que l'agriculture, elle n'a pas et n'aura pas de longtemps son Gasparin ; mais des ouvrages spéciaux sur ses diverses branches ont été publiés à différentes époques avec d'autant plus d'autorité que leurs auteurs avaient limité leur champ d'études. En 1886, nous avons voulu apporter notre contingent à ce vaste ensemble d'une science chère à tous les amis des forêts et nous avons publié les Estimations concernant la propriété forestière, contenant quelques apercus nouveaux.

Aujourd'hui, nous abordons l'étude beaucoup plus importante de l'Économie forestière; mais le livre que nous offrons au bienveillant examen du public forestier n'en forme qu'une faible par'ie.

Voici comment nous concevons le plan d'un traité d'économie forestière :

La première partie doit, à l'imitation de l'économie politique, étudier la production; considérant la forêt comme une fabrique de bois, elle examine l'organisation économique de cette exploitation, le matériel engagé et les produits, l'outillage et le but à atteindre. C'est la conception théorique de l'exploitation forestière qu'on examine dans les différents cas, c'est-à-dire dans chaque mode de traitement cultural.

La seconde partie fait l'application de cette conception théorique à la forêt réelle, c'est-à-dire aux immeubles boisés, tels qu'on les possède, c'est l'aménagement avec ses différents caractères. On concoit que l'idée théorique est le guide nécessaire pour installer une exploitation forestière ayant un but déterminé. Il en est ainsi de toute entreprise industrielle ou agricole.

La troisième partie correspond à celle que l'économie politique connait sous le titre de circulation et distribution 
de la richesse. On y examine la manière dont l'arbre et le peuplement créent la valeur forestière, les lois de cette valeur, sa distribution entre le producteur et le consommateur, les questions d'importation et d'exportation en présence de données fournies par la statistique forestière des différentes nations.

Ce livre ne comprend que des notions préliminaires et la première partie de notre programme; la suite viendra peut-être ultérieurement. Nous nous excusons d'émettre ainsi nos idées sur l'économie forestière à faibles doses et à petites étapes. Les idées nouvelles ne font leur chemin qu'à cette condition. Nous nous excusons également de certaines longueurs de style et d'un exposé parfois trop didactique: on a dit des professeurs qu'ils sont plus soucieux d'enseigner que désireux de plaire au lecteur.

Nancy, le 15 mai 1888.

A. Puton. 

PREMIÈRE PARTIE

NOTIONS PRÉLIMINAIRES 



\section{CHAPITRE I ${ }^{\text {er }}$}

\section{CAUSERIE FORESTIÈRE}

Nos1. - Avantages généranx des placements en forèts : sécurité.

2. - Facilité de leur gestion.

3. - Amélioration graduelle du revenu et de la valeur capitale.

4. - Taux du placement, motifs de sa modération.

5. - Avantages particuliers. - Combinaisons d'exploitation.

6. - La forêt, caisse de dépôt et d'éparơne.

7. - La forêt, caisse d'escompte.

8. - lmmeuble agréable et intéressant.

9. - Objections. - Faut-il habiter la campagne pour être propriétaire de bois?

10. - Faut-il être riche pour en acheter?

11. - La propriété forestière est-elle assez protégée?

12. - Il faut s'y connaître pour les administrer.

13. - Conseils pour acheter.

14. - pour administrer.

15. - pour conserver.

16. - Quelques mots pour les agents forestiers.

No 1. - Avantages généraux. - "On voudrait " trouver un placement de capitaux offrant toute garantie " de sécurité, étant d'une administration facile, et sus" ceptible d'une certaine amélioration dans l'avenir; à ces " conditions, on se contenterait d'un taux d'intérêt " modéré. "

Voilà, assurément, un langage que bien des capitalistes et bien des pères de famille ont tenu et que beaucoup de personnes ont entendu tenir autour d'elles, ou pu voir dans les annonces de journanx.

Nous n’hésitons pas à répondre:

"Achetez une forêt!" " 
La sécurité du placement est, en effet, hors de doute : c'est celle des immeubles fonciers. C'est même une sécurité plus grande que celle des fonds agricoles, car les risques des intempéries, telles que la grêle, la gelée, etc., si redoutables aux cultivateurs, $y$ sont très minimes et presque insignifiants. La sécurité est plus grande aussi que celle des immeubles bâtis, soit à la ville, soit à la campagne, car les danger's d'incendie sont, en général, si peu graves que l'assurance devient le plus souvent inutile. Naturellement, il faut savoir choisir la forêt qu'on veut acheter; il en est ainsi de tous les immeubles.

No 2. - Quelle gestion plus facile que celle des forêts! un immeuble qui travaille tout seul. Là, point de labours, de fumures, de drainages ni d'irrigations; rien de ce travail agricole qui oblige à chercher, soit un entrepreneur, soit un métayer, soit enfin un fermier, selon les différents modes d'exploitation usités. L'intermédiaire souvent malfaisant ou mal payant et toujours se plaignant est supprimé, car on ne loue guère de forêts. Le propriétaire gagne la part du produit qui, dans l'agriculture, est la rémunération légitime de l'intermédiaire exploitant. Il ne partage avec personne et la totalité du prix des produits lui est acquise, sauf, bien entendu, la partie de ce prix qui forme le bénéfice du commerce et qui va aux marchands de bois, comme aux marchands de denrées agricoles, quand l'exploitant ne vend pas directement au public consommateur. Or, le propriétaire de forêts peut, comme le propriétaire de champs, être à la fois exploitant et marchand, producteur et vendeur direct. Il a même, pour agir ainsi, bien plus de facilités que le propriétaire agricole, car il n'est pas, comme celui-ci, occupé aux soins incessants de la culture et aux travaux de chaque jour. Un traité pour exploiter la coupe à passer avec des bûcherons, braves gens que l'on retrouvera chaque année, 
parce qu'ils travaillent en morte saison, voilà l'unique travail de la forêt, c'est, en réalité, celui de la récolte seule.

La forêt est un bien naturel. Le meilleur et le plus sûr moyen de restaurer une forêt dégradée est de la laisser reposer et de l'oublier en quelque sorte $\left({ }^{1}\right)$, c'est assez dire la facilité de son administration.

$N^{\circ} 3$. - L'amélioration graduelle du revenu en argent et par suite celle de la valeur capitale d'une forêt ne saurait être mise en doute par personne. La ville de Remiremont (Vosges) possède une sapinière de 900 hectares, dont le plan d'exploitation est réglé sur an produit de 3,000 mètres cubes: en 1825, la ville était heureuse et estimait ses coupes avantageusement vendues, quand Je revenu atteignait 27,000 fr.; en 1875, la même forêt, ayant la même contenance, la même production et le même genre d'exploitation, rapportait $75,000 \mathrm{fl}$. Le prix du mètre cube a passé de 9 à 25 fr. Il n'y a dans cet exemple, ni un fait isolé, ni une anomalie, mais, bien au contraire, l'application d'une loi économique dont chacun a pu se rendre compte. Tous les prix des objets de consommation ont notablement augmenté; cela tient à des causes multiples, parmi lesquelles la plus caractéristique est l'énorme augmentation des métaux précieux dont les quantités extraites de la terre vont toujours en croissant. Le signe monétaire a baissé, non de prix officiel et marqué, mais de valeur réelle. Il est bien certain qu'on obtenait, il n'y a pas bien longtemps, beaucoup plus de services et de choses avec 25 grammes d'argent qu'on ne pent s'en procurer maintenant. Le prix nominal des

( $\left.{ }^{1}\right)$ Ce moyen, que les ordonnances de réformation appelaient mises en ban ou en défens, paraît même avoir été le seul mode de restauration connu des anciens forestiers. 
mêmes objets a donc beaucoup augmenté. On soutiendrait vainement que l'effet est produit, que les prix des objets de consommation tendent maintenant à se niveler par l'effet des chemins de fer et des moyens rapides de transport, et qu'ils n'augmenteront plus notablement. Que ce nivellement existe, qu'il soit même de nature à retarder, dans une certaine mesure, l'augmentation des prix, qu'il y ait des moments d'arrêt et des fléchissements temporaires dans ces prix, nous le reconnaissons, mais cette augmentation est fatale tant que ne seront pas épuisées les mines d'or et d'argent de l'Oural, de l'Australie, du Mexique et de la Californie.

Si donc les prix vont toujours en augmentant, avec l'abaissement de valeur du signe monétaire, le propriétaire de forêts verra son revenu s'améliorer progressivement, et avec lui la valeur capitale de son imrneuble. Sans aucun doute, cette amélioration ne sera pas sensible d'une année à l'autre, mais elle sera forcément acquise dans une période de temps plus ou moins longue, et ses enfants en profiteront sûrement.

En tout cas, le propriétaire de forêts a un avantage qu'on rencontre rarement dans la vie : celui d'être à la fois producteur et consommateur, avantage considérable que les ouvriers seuls possèdent et qui les rend justement insensibles à l'augmentation dı prix de la vie. Que de gens, que de rentiers ne sont que consommateurs et se trouvent un jour au dépourvu avec leurs rentes fixes et leurs fermages dépréciés! Sans doute, le propriétaire agricole, qui exploite directement sa ter're et la fait valoir par lui-même, au lieu de la louer, est aussi consommateur et producteur; mais pour lui cet avantage est tellement minime qu'il devient en quelque sorte insensible. L'augmentation des objets nécessaires à la vie a fait tant hausser le prix des salaires que si les produits de l'agriculture augmentent de prix, le bénéfice de cette augmentation va 
à la main-d'œurre et ne laisse que fort peu de chose à l'agriculteur. Dans la forêt, au contraire, le salaire n'influe que sur la récolte; le propriétaire, libre de son temps, peut consacrer son travail à d'autres occupations; son revenu suit, à son insu et sans crises pour sa famille, la valeur de toutes les choses de la vie.

Une autre considération contribue encore à assurer dans l'avenir l'augmentation de la valeur des produits et des immeubles forestiers. Ni l'emploi du fer dans les constructions, ni celui de la houille pour le chauffage, n'ont un seul instant ralenti la marche progressive du prix des bois. - Tous les consommateurs le savent trop pour qu'il soit nécessaire d'en faire la démonstration, cela tient à l'insuffisance des forêts dans notre pays.

Ici les chiffres relevés par la Statistique des forêts, publiée en 1878, ont leur éloquence :

Nous avons, en France, 9,185,310 hectares de forêts, soit 17 pour cent du territoire (17 ares boisés par hectare) et 25 ares par habitant sur une population moyenne de 68 têtes par cent hectares. A première vue, cela paraît très satisfaisant et nous sommes encore avec le $\mathrm{n}^{\circ} 8$ sur la liste des 15 États européens dont on connait les ressources forestières. Mais relativement aux ressources en bois de chauffage et de service que ces 25 ares peuvent fournir à chaque habitant, la quantité devient tout à fait insuffisante.

La statistique de 1878 évalue, en effet, le produit de l'année 1876 :

en matière à 25,342,440 mètres cubes en argent à 236,755,430 francs $\left({ }^{1}\right)$,

$\left({ }^{1}\right)$ Ce chiffre est le revenu des forêts, c'est-à-dire la portion dı prix des bois qui rerient au propriétaire. Le prix payé par le consommateur est naturellement grossi des frais de façon et de transport et doit être éralué à plus de 400 millions. 
soit par hectare et par an :

$$
\begin{aligned}
& \text { en volume } 2^{\text {mc }}, 75 \text { ) } \\
& \text { en argent } 25^{\text {f }} 75
\end{aligned}
$$

De sorte que chaque habitant n'a pour ses besoins annuels en bois que :

$$
\begin{array}{ll}
\text { un volume de } & 0^{\mathrm{mc}}, 787 \\
\text { une valeur de } & 6^{\mathrm{f}} 45
\end{array}
$$

Encore convient-il de remarquer que dans cette production de $2^{\mathrm{mc}} 3 / 4$ par hectare de forêt, il y a $2^{\mathrm{mc}} 1 / 4$ de bois de feu et $1 / 2$ mètre cube de bois de service, soit par tête d'habitant environ 6 dixièmes de mètre cube pour le chauffage et un peu plus d'un dixième de mètre cube pour les vastes besoins des constructions privées et publiques, civiles et navales, du travail sous ses mille formes et des innombrables traverses de nos voies ferrées.

Aussi sommes-nous largement tributaires des pays voisins : en 1850, nous achètions de l'étranger pour 50 millions de bois et nous lui en vendions pour 4 millions et demi.

En 1883, nous avons importé $271,600,000$ francs de bois et exporté 28,200,000 francs; nous avons eu besoin de près de 243 millions de bois que la consommation du pays n'a pu trouver dans la production des forêts francaises $\left({ }^{1}\right)$.

Le bois est une marchandise que dans nos sociétés modernes, actives à l'excès, on use plus vite qu'on ne la fabrique, et il n'y a aucune témérité à promettre aux propriétaires de forêts une augmentation certaine de revenus et de valeur capitale dans l'avenir.

Cette augmentation sera acquise, le plus sûrement à

( $\left.{ }^{1}\right)$ Valeur dite actuelle, c'est-à-dire prix de consommation. Anmuaire des eaux et forêts, 1883 . 
ceux.qui, sans renoncer, bien entendu, à produire du bois de feu (on ne saurait faire autrement) auront la sagesse de reculer le terme de leur exploitation et surtout de :

\section{Faire des Arbres!}

car, ces 243 millions de francs en bois importés sont principalement des bois de construction et de travail, dont notre production est la plus pauvre.

Une pareille importation ne peut toujours grandir. L'étranger n'a point de mines inépuisables de bois de service; le nombre de ceux-ci diminuera chez nos roisins comme chez nous : l'avenir est au plus sage, c'est-à-dire à celui qui saura conserver des arbres!

$N^{\circ}$ 4. - Certes, on peut se contenter d'un taux de placement modeste pour un immeuble dont les revenus et la valeur capitale sont ainsi assurés d'une amélioration future. A cet égard, et sans trop anticiper sur le terrain de l'économie forestière, nous pouvons dire que les fonds engagés dans l'achat d'une forêt sont susceptibles de taux de placement très variables de 4 pour cent à 2 et même 1 pour cent. Si on exploite à court terme, c'est-à-dire à des âges peu avancés, le taux du placement est le plus élevé; il s'abaisse à mesure que l'âg'e d'exploitation est reculé et qu'on laisse vieillir les arbres ou les peuplements.

Ainsi voilà un mode de placement très commode et qui fournit au capitaliste plus qu'on ne lui arait promis, puisqu'il le laisse libre de choisir le taux d'intérêt qui convient à sa fortune et aux exigences de sa vie.

Or ici, il convient de s'entendre et de ramener les choses à la réalité :

Lorsque pour acheter une maison, on calcule le prix en multipliant le loyer par 20, lorsque pour acquérir une ferme bâtie on en donne 25 fois la location, lorsque, enfin, 
le prix de terres arables ou de prés se règle sur 33 fois le fermage, on dit généralement que :

La maison rapporte 5 pour cent.

La ferme - 4

Les terres - 3

c'est une illusion dont l'acquéreur sera bientôt revenu, quand, au bout d'un certain nombre d'années, il aura défalqué du produit brut de la maison, les frais d'assurance contre l'incendie, la contribution supplémentaire de la propriété bâtie, les réparations demandées par les locataires de plus en plus exigeants et les pertes de loyers. Pour la ferme, les réparations sont moins chères, les contributions moins onéreuses, c'est pour cela que le taux des achats s'abaisse. Il diminue également pour les terres, parce qu'il n'y a plus ni assurance, ni impôts de bâtiments, ni réparations; mais il y a encore les défauts de payement des fermiers, qui mettent le taux du placement au-dessous de 3 pour cent. Tout compte fait et établi sur une certaine période de temps, on remarque que le taux des immeubles se nivèle, ou tend, tout au moins, à se niveler.

$\mathrm{Au}$ propriétaire de forêts, à celui qui veut en acheter nous disons donc: ne vous laissez pas séduire par ce mirage de taux plus ou moins élevés dans les immeubles ; ayez la sagesse de ne demander à votre forêt et à l'acquisition que vous voulez faire qu'un taux modéré, inférieur même à celui qui est le plus faible pour les terres non bâties. Vous êtes certain, en effet, de n'avoir ni faillite de fermier, ni réparations à subir. Si votre revenu paraît faible, par rapport au capital engagé, c'est-à-dire à la valeur de la forêt ou à son prix d'achat, rous pouvez calculer votre vie sur des données certaines, sans avoir à subir ces désagréables surprises que réservent les grosses réparations et les défaillances des fermiers, qui abaissent le taux du placement avec cette circonstance aggravante, 
que souvent elles emportent toute une année de revenu, sans rien laisser pour les besoins de votre ménage.

Ajoutez à cela que les forêts, ayant pou de voisins, ont peu de procès, que la charrue des anticipateurs les entame difficilement, que les gelées, grêles et intempéries ont peu d'action sur elles et vous aurez bien des motifs pour vous contenter d'un faible taux de placement, et pour vous expliquer que certains propriétaires trouvent encore leur compte dans un revenu en apparence très minime, relatirement au capital représenté par leur forêt.

Ainsi le taux des placements en forêts est généralement assez faible parce qu'il est net des pertes habituelles aux autres immeubles; mais il n'est pas pour cela fixe et constant; il comporte, au contraire, des variations et s'abaisse à mesure que la ricliesse de la forêt augmente et que l'âge d'exploitation s'accroît, de sorte qu'il y a des placements en forêts pour tous les goûts et pour toutes les positions de fortune.

$\mathrm{N}^{0}$ 5. - Avantages particuliers. - Sécurité du placement, - facilité de gestion,- amélioration progressive du revenu et de la valeur capitale, - taux d'intérêt modéré mais très convenable, - la forêt donne et assure tout cela! Ce remarquable immeuble n'est pas avare d'avantages; il en fournit bien d'autres dont il nous reste à parler.

En première ligne, nous mettons les combinaisons multiples que la forêt permet de créer en vue de tous les besoins de la vie. Laissez croître un ou plusieurs hectares de forêts, les pousses s'ajouteront aux pousses, la fabrication nouvelle s'unira au bois fabriqué, vous aurez une vé. ritable caisse de capitalisation, aussi sûre que celle de nos plus puissantes compagnies d'assurances, s'ouvrant sans limite aux petites et aux grosses mises de fonds, travaillant avec la puissance de l'intérêt composé, sans relûche 
et sans arrêt, s'adaptant à merveille à la formation d'une dot, ou d'un capital à créer en vue d'un établissement.

Groupez, au contraire, un certain nombre d'hectares, trente par exemple, dont vous exploiterez un chaque année: rous aurez un immeuble qui vous donnera des produits anmuels adaptés aux besoins de votre vie et de volre ménage, et qui vous les donnera, si vous savez bien l'administrer, avec une régularité aussi grande que celle des fermages de l'agriculture.

Dans ces deux conceptions se trouve une distinction fondamentale entre les exploitations de forêts, distinction que nous retrouverons constamment dans la suite, les exploitations périodiques et les exploitations aménagées. La forêt est un immeuble dontles produits sont périodiques de leur nature. On attend que les tiges aient de la valeur ou qu'elles aient atteint une valeur déterminée; on les coupe et on recommence à nouveau. C'est une différence caractéristique avec les terres agricoles: champs ou prés livrent leurs produits tous les ans; les forêts ne les livrent qu'à des intervalles souvent fort longs. On ne peut en obtenir des produits annuels que par des groupements, des combinaisons de contenances ou de matériel qui constituent ce que l'on appelle l'aménagement, mot très bien dérivé de son étymologie: adaptation aux besoins du ménage; combinaison qui a pour but de rendre annuels les produits d'un immeuble, qui sont de leur nature périodiques.

Mais à cela ne se borne pas les services que le propriétaire de forêts peut demander à son immeuble.

Veut-il, par exemple, imiter ces sages réseres que les compagnies financières font sur leurs revenus pour augmenter leur puissance ou pour parer à des besoins imprévus? Il le pourra sans sortir de sa forêt, sans s'adresser à des institutions de crédit, et de plusieurs manières. Il pourra, par exemple, n'exploiter que $9 / 10$ de sa coupe, si 
la forêt est aménageée; il pourra aussi joindre à sa forêt aménagée, quelques hectares à exploitation périodique; il pourra constituer sa réserve sur le volume ou sur la contenance, à assiette mobile ou fixe, dans la proportion qu'il lui plaira d'adopter et suivant des procédés que nous indiquerons dans la suite, car nous n'avons pour but ici que de laisser entrevoir les combinaisons fécondes offertes par la propriété boisée. Elles constituent un avantage spécial, dont ne jouissent point les exploitations agricoles. Le cultivateur peut bien disposer sa ferme dans un but déterminé, disposition qu'il appelle aussi aménagement, mais dans un sens tout autre, car ses produits sont toujours annuels. S'il veut se constituer des réserves dans la véritable acception de ce mot, c'est-à-dire des ressources toujours disponibles, il est obligé de s'adresser à des institutions de crédit ou de clépôt et de les placer en dehors de chez lui. Le propriétaire forestier peut ne jamais sortir de sa forêt.

No 6. - Nous avons parlé de la forêt envisagée comme caisse de capitalisation des économies que le propriétaire peut faire sur son revenu. Il y a, de ce côté, un avantage qu'on ne saurait trop mettre en lumière, et qui permet de trouver dans la forêt une véritable caisse d'épargne: le propriétaire de bois peut placer dans sa propre forêt toutes les épargnes qu’il pourra faire en dehors sur son travail ou ses autres revenus et cela d'une facon à peu près illimitée, pour les plus petites comme pour les plus grosses sommes, pour le temps le plus court comme pour le plus long. Cela tient à cette différence caractéristique qui existe entre les champs et les bois : non seulement les produits de l'agriculture sont annuels, mais ils doivent être impérieusement récoltés chaque année sous peine de perte. La coupe des arbres peut, au contraire, être longuement retardée. 
L'épargne que l'on veut faire vient-elle de la récolte, c'est-à-dire d'une coupe qui dépasse les besoins du propriétaire? Qu'il laisse sur pied les arbres excédant ses besoins: la forêt sera son grenier... grenier bien commode, car il ne coûtera rien à établir; la récolte, loin d'y dépérir, continuera à vivre et à s'accroître.

L'épargne vient-elle d'une source extérieure? qu'au lieu de la porter à une banque de dépôt, le propriétaire la place encore dans sa forêt; car là, comme à la banque, elle produira ses intérêts à un taux que le forestier apprendra à déterminer et que nous pouvons, dès à présent, dire très avantageux.

Une année de crise abaisse momentanément le prix du bois, comme cela peut arriver à toutes les récoltes. Le propriétaire agricole devra réaliser quand même; ses produits n'attendent pas. Le propriétaire de forêts pourra retarder sa coupe ou n'en exploiter que moitié pour attendre un meilleur moment.

Est-ce là un si minime avantage, qu'il soit inutile d'en parler?

Ainsi, caisse de dépôts momentanés et caisse d'épargne permanente, fonctionnant toutes deux à bons intérêts; voilà le rôle éminemment utile de la forêt dans le patrimoine d'une famille.

Qu'on ne nous accuse pas d'exag'ération ou d'enthousiasme professionnel.

Deux exemples, que chacun pourra rérifier, suffiront à éclairer sur l'effet des petites économies placées dans la forét.

$1^{\circ}$ Le propriétaire d'un taillis de trente ans laisse sur pied dans sa coupe 100 beaux brins de 30 ans; ils valent 25 centimes pièce, 25 francs en tout. L'économie n'est pas forte et le sacrifice qu'elle impose n'est pas lourd à supporter, mais à la coupe suivante, c'est-à-dire dans 30 ans, ces brins de 60 ans raudront chacun 3 francs, et les en- 
fants trouveront leur revenu amélioré de 300 francs. Estce à dédaigner ?

$2^{\circ}$ Dans une sapinière des Vosges, le propriétaire est en face d'un beau sapin de $0^{\mathrm{m}}, 50$; il l'estime 50 francs et le laisse en épargne sur sa coupe. Quinze ans après, le sapin a grossi de $0^{\mathrm{m}}, 10$; il a $0^{\mathrm{m}}, 60$ de diamètre et il vaut 100 francs! Ce propriétaire où sa famille se plaindrontils? S'ils doutent du fait, ils n'ont qu'à aller dans les sapinières de l'Est; ils constateront qu'un sapin de $0^{\mathrm{m}}, 50$ double de valeur en passant a $0^{\mathrm{m}}, 60$ de diamètre.

Dans le premier exemple, la caisse d'épargne a fonctionné à raison de $81 / 2$ pour cent à intérêts composés; dans le second, la capitalisation s'est faite à 4,7 pour cent d'intérêts également composés.

Où pourrait-on trouver meilleur et plus solide banquier?

$\mathrm{N}^{\circ}$ 7. - Le cultivateur ne peut pas plus devancer l'époque de sa récolte qu'il ne peut la retarder; le forestier peut toujours anticiper ses coupes. C'est encore là une différence notable. Que ces anticipations soient de bonne pratique, cela est douteux. Que ces emprunts faits au matériel de la forêt aient pour effet de la dégrader parfois et de la compromettre souvent, nous sommes des premiers à l'affirmer. Il en est ainsi chaque fois qu'on emprunte à sa fortune, mais on ne saurait se refuser à reconnaître qu'il y a daus la vie des circonstances urgentes où il faut trouver cles ressources; - qu'il est presque toujours moins onéreux d'emprunter à soi-même qu'à autrui; - qu'anticiper une coupe, c'est souvent jrofiter des hauts cours sur le marché des bois. On ne peut nier qu'il y a dans cette faculté, dans cette sorte de caisse d'escompte, à court et à long terme, un avantage qu'aucune autr'e propriété rurale ne saurait offrir à son propriétaire.

Le cultivateur qui a besoin d'argent ne peut que gager 
sa récolte en attendant sa maturité. Le propriétaire de forêts peut battre monnaie à toute époque. Le gage sans nantissement sur récoltes pendantes n'a pas encore trouvé sa forme légale pour le crédit du cultivateur. Le forestier peut à la rigueur s'en passer ; s'il a été réclamé pour lui, c'est autant au nom de l'intérêt public qu'au sien propre, car la société a intérêt à ce que des réalisations de bois sur pied ne soient pas faites avant l'époque de leur maturité, c'est-à-dire avant le moment où ils rendent le plus de services au travail du pays.

Le seul revers à cette médaille, c'est que la grande facilité de mobilisation du matériel des forêts diminue pour les propriétaires de bois l'étendue de leur crédit hypothécaire; on prête moins par hypothèque sur une forêt que sur des terres de valeur égale, car le débiteur peut réaliser les bois sur pied et diminuer le gage du créancier - les meubles n'ayant pas de suite, suivant le langage du droit.

La forèt ne saurait avoir tous les avantages et il faut se résigner aux petits inconvénients nés de ses qualités; c'est pour cela que le crédit agricole, constitué sur le gage sans nantissement de ces objets qui sont, comme les récoltes et les bois, mobitisables, c'est-à-dire intermédiaires entre les meubles et les immeubles de notre droit civil, pourra avoir une réritable utilité pour les propriétaires de forêts. Quand ce nouvel instrument de crédit aura été légalement organisé, les forêts n'auront, sous ce rapport, que des avantages pour leurs propriétaires $\left({ }^{1}\right)$.

No 8. - Nous ne saurions citer pour simple mémoire un autre côté des avantages d'un placement de capitaux en forêts. Il est possible que certains propriétaires comp-

(1) Voir notre étude : "Les forèts et le crédit agricole ". Revue des eaux et forêtls, 1883. 
tent pour peu leurs fraîches senteurs, leurs poétiques détours et leurs ombreuses retraites. Les beaux arbres ont cependant leur charme; mais d'autres ne compteront pas pour rien les plaisirs de la chasse que la loi du 22 janvier 1874 a permis de leur prolonger quand elle est fermée aux propriétaires agricoles.

D'autres plus positifs auront réel plaisir à faire des places d'essai dans leurs peuplements, à mesurer des arbres pour en contrôler l'accroissement, à étudier des moyens de transports, à suivre le travail des bûcherons pour saisir le secret du commerce des bois et se rendre compte des débits les plus avantageux. Rien n'est plus intéressant, ni plus attachant, alors surtout que l'intérêt du propriétaire en recoit satisfaction.

Que le propriétaire prenne soin de mesurer quelques arbres en les ceinturant avec de la peinture pour que les mesures ultérieures soient faites à la même hauteur, il ne tardera pas à les aimer en constatant la manière dont ils traviillent pour lui; qu'il s'occupe à installer des emplacements destinés à étudier les effets dus à l'écartement des tiges, à l'enlèvernent des feuilles mortes et de la couverture du sol, au débroussaillement des tiges et arbustes vivant en sous-étage, la forêt lui livrera d'intéressantes observations comme le champ d'expériences en fournit à l'agriculteur.

L'étude de la nature vivante est une des plus attrayantes occupations qu'on puisse rencontrer: elle explique l'amour passionné que les forestiers ont pour leur profession.

No 9. - Objections. - Nous avons énuméré tous les avantages des placements en forêts d'une manière sommaire et générale, sauf à préciser ultérieurement les faits et en donner la justification raisonnée. Il est cependant un fait expérimental certainement digne 
de remarque, c'est l'amour que les anciennes communautés religieuses avaient pour les forêts. Leur charme poétique a été d'un faible poids dans les considérations qui ont décidé ces sociétés à y placer leurs capitaux; elles y ont trouvé, avec des revenus assurés, le placement de leurs épargnes et l'augmentation de leur patrimoine, avec cette agréable condition que la forêt travaillait seule à les enrichir. Nous avouons ne pas comprendre comment les sociétés financières, telles que les compagnies d'assurances, en quête de placements de cette solidité et de cette nature, n'y emploient pas plus de capitaux; - comment nos établissements publics, hospices, bureaux de bienfaisance, n'imitent pas davantage la sagesse des anciennes communautés religieuses $\left({ }^{1}\right)$; - comment, enfin, tant de familles soucieuses de leur avenir ne cherchent pas dans ce mode de placement la garantie de leur rang dans la société.

C'est salis doute parce qu'on fait à ce mode d'emploi des capitaux des objections nombreuses. Il faut, dit-on, habiter la campagne, - il faut être riche pour avoir des forêts, - celles-ci n’ont pas dans la législation une protection suffisante, - et enfin, il faut s’y connaître pour les administrer convenablement.

Examinons successivement ces objections.

S’il est une propriété foncière dont la gestion n'exige pas l'habitation à la campagne, c'est certainement celle des forêts, puisque la main de l'homme est étrangère à la fabrication des produits et qu'elle n'intervient que pour leur réalisation : quelques visites par an pour marquer la coupe, la vérifier quand elle est exploitée, dénombrer les produits fabriqués par les bûcherons, visites

( ${ }^{1}$ Il n'y a en France que 32,059 hectares de forêts appartenant à 1,175 établissements publics. Ce sont de petites furêts dont l'étendue moyenne ne dépasse pas 52 hectares (Statistique forestière de 1878). 
hygiéniques, agréables et salubres pour l'habitant d'une ville; voilà en quoi se résume le déplacement d’un propriétaire, que les chemins de fer facilitent maintenant de toutes facons. Si, même, la coupe est vendue sur pied à un marchand qui se charge de l'exploitation, le déplacement pourra se réduire à deux opérations, la marque et le récolement des coupes. Est-il un immeuble moins exigeant? Faut-il pour cela habiter la campagne? Même en vendant ses produits au détail, le propriétaire exploitant n'aura pas besoin d'y consacrer beaucoup de temps, si sa coupe façonnée est ouverte aux acheteurs le même jour de chaque année. Le public en prendra vite l'habitude; il saura qu'au même jour le propriétaire viendra lui-même vendre ses bois; en quelques heures, les marchés sont faits avec des clients accoutumés; s'il reste quelques produits invendus, le garde aura mission de les céder au prix moyen $\left({ }^{1}\right)$.

Voilà le maximum des exigences que la gestion d'une forêt imposera à un propriétaire urbain. Nous avons la conviction que l'attrait de l'exploitation forestière l'amènera plus souvent visiter ses bois où il trouvera plaisir et santé.

$N^{\circ} 10$. - On répète continuellement que « Il faut être riche pour acheter des bois! "C'est une erreur complète et trop accréditée. La propriété forestière est plus divisible qu'on ne le croit généralement. Les g’ens de la campagne, fort avisés quand il s'agit de leurs intérêts, savent très bien acquérir des bois de petite étendue pour leur fournir chaque année le chauffage de la maison et, de

( ${ }^{1}$ Les lois du 22 pluviòse an VII et 5 juin $185 \check{1}$, qui réservent aux officiers ministériels les ventes aux enchères publiques de récoltes et de bois, ne sont pas applicables à ces ventes amiables, faites sans publicité annoncée, aux clients accoutumés d'une forêt. 
temps à autre, des pièces de construction pour la réparation des bâtiments.

La question dépend un peu du but que l'on se propose: si l'on veut acheter un bois pour le laisser croître en vue de la constitution future d'un capital, il faudra certainement posséder déjà une certaine aisance, mais il faut cette aisance pour toute épargne et le bois n'y est pour rien.

Si, au contraire, on désire posséder un bois pour en tirer un revenu annuel, il ne faut pas y mettre un capital plus considérable que celui qu'il est dans l'usage de consacrer à l'acquisition d'une ferme Il y a des fermes de toute valeur et de toute importance. Il y a, de même, des bois de toute nature: entre l'hectare de haute futaie de chêne, valant près de 30,000 francs dans le Blaisois, et l'hectare de modeste taillis coûtant à peine 500 francs, il y a toutes les gradations et tous les intermédiaires. Les combinaisons d'aménagement sont très différentes suivant la nature des forêts: avec 30 hectares de taillis sous futaie, exploités à $30 \mathrm{ans}$, on peut avoir un hectare de coupe par an et ne pas y affecter plus de 30,000 francs; on a une série de futaie aménagée en jardinage avec quelques hectares de sapinière. Il y a des bois pour toutes les bourses!

Nous pouvons même noter ici une autre supériorité des forêts sur les exploitations agricoles. Une ferme est habituellement un ensemble constitué d'une manière fixe. On ne peut y ajouter que des terres voisines ou dont l'éloignement ne rend pas le travail trop onéreux; toujours il faut augmenter les bâtiments. L'exploitation forestière n'a pas les mêmes exig'ences; son aménagement peut se constituer par des achats successifs et avec des cantons de forêts très éloignés les uns des autres sans que le propriétaire ait à se préoccuper de bâliments, d'assolements ni de perte sur la main-d'œuvre. 
$\mathrm{N}^{\circ}$ 11. - Le ministère public, dit-on encore, ne poursuit pas d'office les délits forestiers, et la propriété boisée laissée sans protection est exposée à toutes les rapines des populations, rapines journalières, incessantes et finissant à la longue par dépeupler des cantons entiers. De ce côté, il y a du vrai, mais en même temps une exagération certaine et même une faute de la part des propriétaires de bois. D'abord, nulle part, dans nos lois, le ministère public n'est tenu à poursuivre les délits d'aucune sorte; son action est toujours essentiellement libre ; s'il poursuit d'office les infractions graves, c'est que leur existence trouble l'ordre dans la société et légitime son action spontanée. Il attend et doit attendre que les parties lésées lui dénoncent les faits peu graves, tels que les délits de chasse, les infractions forestières ou champêtres. Que les propriétaires victimes d'un délit fassent constater les dégâts soit par le garde particulier qu'ils sont autorisés à instituer (C. for. art. 117), soit par le garde champêtre (') à défaut de garde particulier (C. for. 188); qu'ils adressent le procès-verbal au Procureur de la République (C. for . 191) et ils auront toute répression s'ils se joignent au ministère public à titre de partie civile.

Nous n'avons pas ici à faire un exposé de la législation forestière; elle demande une étude attentive des propriétaires de bois $\left({ }^{2}\right)$; mais nous devons constater que sur beaucoup de points elle est bien plus énergique ef bien plus protectrice que celle des immeubles ruraux.

Ainsi nombre de faits, tels que l'introduction des voi-

(1) Les eonstatations faites par les gardes champêtres sont gratuites et les procès-verbaux qu'ils rédigent à la requête des particuliers sont même enregistrés en débet, e'est-à-dire sans rien débourser (C. for. 188).

$\left({ }^{2}\right)$ Consulter : Sirey, Code forestier annoté, 1 vol. gr. in-8 ${ }^{\circ}, 1867 .-$ Dalloz, Code forestier annoté, 1 vol.in-40, 1884. - Dalloz, Répertoire, vo Forêts, 1 vol. in-4 ${ }^{\circ}, 1849$. - Puton, Manuel de législation forestière, 1 vol. in-12, 1876. - Puton, Code de la législation foresticre, 1 vol. in-12, 1883. 
tures (C. for. 147), des instruments à couper le bois (C. for. 146), l'enlèvement et même le seul déplacement du moindre produit du sol forestier (C. for. 144) qui, dans les champs ne sont protégés que par une action civile, lente et coûteuse à mettre en mouvement, sont érigés dans les bois à l'état de délits; disposition éminemment favorable, car elle donne aux propriétaires de bois, la juridiction rapide et facile des tribunaux répressifs, et leur permet même de réprimer les anticipations des riverains par l'action née du délit, sans avoir recours à l'action possessoire.

Ajoutons à cela que les gardes des particuliers ont le droit de saisie et de visite domiciliaire (C. for. 161-169), de constatation par des procès-verbaux faisant foi jusqu'à preuve contraire (C. for. 188), ce qui les dispense le plus souvent de venir en témoins à l'audience; - que les tribunaux n'ont pas la faculté de modérer les peines par l'admission des circonstances atténuantes (C. for. 203); - que, enfin, les propriétaires lésés par un délit n’ont pas à faire preuve de l'importance du commage éprouvé, car une loi prévoyante leur alloue des dommages-intérêts dont le chiffre ne peut descendre au-dessous de l'amende encourue (C. for. 202).

On voudra bien reconnaître que la législation forestière n'est pas si avare de protection qu'on se plaît trop à lę dire.

Il ne faut pas, du reste, s'exagérer l'importance des délits : dans les conditions habituelles, c'est-à-dire en dehors du voisinage des villes ou des établissements industriels, les rapines de la population agricole portent surtout sur des brins dépérissants, dominés, destinés à disparaître et sans valeur réelle. Avec un peu de fermeté, on en a vite raison. Si la propriété n'est pas assez grande pour comporter un garde particulier, on peut presque toujours obtenir de l'Administration forestière l'autorisa- 
tion d'en confier la surveillance à un des gardes communaux ou domaniaux du voisinage. Il suffit le plus souvent qu’on sache la propriété gardée pour qu'on la respecte.

$\mathrm{N}^{\circ}$ 12. - “ Il faut s’y connaître pour acheter et administrer une forêt. " Ah! nous le reconnaissons, il faut au propriétaire de forêts une certaine instruction technique et nous pourrions citer bien des personnes victimes de leur ignorance, bien des familles tristement réveillées de leur quiétude et d'une confiance aveugle en leur régisseur.

La nécessité d'une instruction professionnelle n'a rien de spécial aux forêts. N'est pas industriel sans danger le fils d'un fabricant qui, s'endormant dans la prospérité acquise par ses devanciers, se désintéresse des progrès réalisés dans l'industrie. Le commerçant a besoin d'un long apprentissage ; l'agriculture elle-même a quitté l'ancienne pratique qui l'avait fait vivre pour devenir une industrie d'autant plus compliquée qu'elle a besoin de faire appel à toutes les branches de nos connaissances.

L'instruction du forestier a ceci d'agréable qu'elle peut s'acquérir en peu de temps, par soi-même, sans apprentissage à la ferme ou à l'atelier. Les grandes lignes de la théorie forestière s'étudient, en effet, au cabinet, dans des traités spéciaux. La sylviculture, cette application aux forêts des données expérimentales des sciences naturelles, est commandée par une règle de bon sens qu'il ne faut jamais perdre de vue : "Observez la nature, ne la contrariez jamais ", c'est la règle de tous les biens naturels dont la formation est indépendante de l'homme. Quant à l'utilisation et au clébit des produits, un peu d'observation attentive du travail des bûcherons et des habitudes du commerce suffira pour s'en rendre compte.

N'est-ce pas une qualité précieuse de l'exploitation d'une forêt que de pouvoir l'étudier ainsi soi-même et la diriger 
sans prendre à ses habitudes de ville ou au travail de sa fonction d'autre temps que celui réclamé par une salutaire hygiène!

Nous savons que la théorie des forêts a été quelquefois voilée sous des nébulosités doctrinaires, que le coup l’œil du forestier a été souvent érig'é à l'état de prescience innée et fatalement nécessaire. Nous nous appliquerons dans ce Traité d'Économie forestière à être surtout clair et positif, à n'employer que le calcul accessible à tous, à nous affranchir, enfin, de ces idées abstraites dont toute science aime à s'entourer à ses débuts, pour revenir enfin à la règle simple et pratique. Que le coup d'œil expérimental ait utilement servi des gens sans instruction, cela est incontestable et cela se rencontre dans tous les métiers, dans les plus compliqués comme dans les plus humbles. On voit de simples toucheurs de bestiaux indiquer sans se tromper le poids d'un animal vivant; mais on voit aussi des agriculteurs instruits arriver au même résultat par l'étude raisonnée et des propriétaires de forêts estimer le volume et la valeur d'un arbre avec autant de sûreté qu'un vieux bûcheron ou un bon facteur de coupe.

Il ne faut pas croire qu'en proclamant ici, haut et fort, la nécessité de l'étude pour un propriétaire de bois, nous n’avons en vue qu'une étude rapide, élémentaire et provenant d'un simple manuel ou traité forestier. Il faut au propriétaire beaucoup plus que cela; il aura besoin de connaissances sérieuses en droit général et spécial, d’études g'éologiques et chimiques du sol, d’études botaniques et physiologiques des végétaux, d'arpentage, de constructions, de sylviculture et de technologie forestière. Nous sommes à l'époque où il faut beaucoup savoir dans tous les métiers et où l'instruction générale et professionnelle s'impose comme le meilleur aide de la vie. Tout ce que nous voulons dire, c'est que les connaissances utiles au propriétaire forestier sont intéressantes ct agréables à étu- 
dier, toujours profitables à ses intérêts et faciles à acquérir pour l'homme du monde qui, n'ayant pas suivi les cours d'une école forestière, voudra sérieusement s'en donner la peine.

$\mathrm{N}^{\circ}$ 13. - Conseils. - Nous avons répondu aux objections; nous nous permettons maintenant de donner quelques conseils à ceux qui achètent des forêts ou qui en possèdent, conseils que nous rendrons aussi brefs que possible, car il faudrait pour les expliquer complètement, entrer trop avant dans des matières techniques où nous ne voulons faire que des incursions incidentes.

$1^{\circ} N^{\prime}$ achetez qu'une forêt faite, c'est-à-dire bien pleine, fertile et vivace, sans vides à repeupler, sans dégradations à réparer. Les repeuplements artificiels sont trop coûteux par nos temps de cherté dans les salaires; ils n’ont été réellement avantageux que dans les r'égions particulières, telles que la Sologgne ou les Landes, où la terre se refuse à tonte autre production que celle du bois, et où la forêt plantée forme, par ses essences, une culture forestière en quelque sorte spéciale. La forêt à restaurer perd toutes ses qualités de bien naturel.

$2^{\circ} \mathrm{N}^{\prime}$ achetez pas de forêt grevée de droits d'usages en bois ou en pâturage, au profit d'une communauté d'habitants, ou au profit d'un domaine particulier; vous n'en auriez que des désagréments continuels se traduisant en concessions ou en procès, en pertes d'argent ou de temps. Si vous voulez dégrever votre immeuble de ces droits par le cantonnement ou par le rachat (C. for., art. 63 et 64), vous payerez toujours plus cher que vous n'avez compté et d'autant plus que vous retarderez.

$3^{\circ}$ Achetez toujours votre forêt dans des régions bien servies en chemins, routes et voies ferrées, dans lesquelles le commerce des bois est établi et la valeur créée. Vous payerez votre acquisition plus cher, mais vous vendrez 
ros coupes en conséquence; les prévisions de vos plans d'exploitation s'accompliront et vous n'aurez jamais ni déboires ni mécomptes. Acquérir une forêt dans une région déshéritée et où la valeur n'est pas créée, dans la pensée qu'un jour viendra où les produits se vendront à bon prix, c'est prendre un billet à la loterie et en attendre le numéro gagnant. Il est digne de remarque que le déboisement des régions du plateau central de la France est dû à l'absence de commerce et de moyens de transport. Le pâturage a détruit sans peine la forêt qui était sans valeur. Si l'Auvergne se reboise depuis 40 ans, c'est que les routes y ont introduit l'activité sociale. Les environs de Paris, les départements du Nord et du Nord-Est ont toujours été convenablement boisés parce que les besoins de la population et de l'industrie y ont toujours donné la valeur aux produits.

$\mathrm{N}^{\circ} 14$. - Voici également quelques conseils, quand la forêt sera achetée :

$1^{\circ}$ Administrez vous-même votre forêt, ne la confiez pas à un régisseur; un garde honnête et intelligent suffit à cette nature de propriété, dont vous avez tout intérêt à être vous-même l'administrateur. Surtout, marquez vousmême vos coupes et ne les laissez point marteler en dehors de votre présence. La coupe est l'opération capitale de la gestion d'une forêt; par elle on peut ruiner sa richesse en fort peu de temps et d'une manière presque irrémédiable. Dans le service de l'administration des forêts, deux Agents forestiers dont l'un a le grade élevé d'inspecteur, assistent à la marque des coupes, tant est grande l'importance de cette opération pour la conservation et l'avenir de la propriété. Avec un régisseur, on finit par se désintéresser de l'administration de sa forêt et à devenir étranger aux notions les plus élémentaires de l'exploitation. Des gérants payés à tant l'hectare de coupe et dési- 
reux d'augmenter leurs honoraires, ont pu, ainsi, persuader à leurs propriétaires d'abaisser l'âge d'exploitation, ce qui avait pour effet d'augmenter la surface de la coupe et d'accroître temporairement le revenu. Au bout de 15 ou 20 ans, la valeur capitale de l'immeuble avait diminué de moitié et le revenu avait baissé dans une plus forte pro. portion. D'autres, payés à tant pour cent sur le produit des coupes, ont fait abattre tous les beaux arbres qui constituaient la valeur et l'ornement de la forêt. Ailleurs, le propriétaire exigeait de son régisseur un gros revenu tout en lui prescrivant de ne point diminuer le nombre des arbres de réserve. Le gérant marquait de petites tiges comme anciens, c'est-à-dire comme on a l'habitude de marquer les gros arbres de réserve, et comme le propriétaire ne visitait point sa forêt, il ne put constater que trop tard que son immeuble avait tout à fait diminué de valeur. Voilà où on en arrive avec un régisseur!

$2^{\circ}$ Défiez-vous des fantaisies en matière de modes de traitement de votre forêt et ne vous laissez pas séduire par des raisonnements plus ou moins ingénieux sur la comparaison entre le taillis, le jardinage, la futaie pleine, le taillis sous futaie et autres modes de traitement. Du moment où votre forêt est bien remplie, bien garnie d'arbres sur un sol bien courert, l'essentiel est atteint.

Chaque localité a son mode de traitement accommodé à son climat, à ses essences et à son terrain. Changer le traitement local, c'est introduire dans le pays une nouveauté que l'expérience et même la nature n'ont point consacrée ; c'est tenter une entreprise des plus hasardées: c'est comme si l'agriculteur voulait introduire partout en France la culture du blé par la raison qu'elle enrichit la Beauce, ou la pratique des embonches parce qu'elle réussit en Charolais. Chaque localité a son mode cultural commandé par le climat, le sol et l'exposition ; améliorez ce mode de traitement local, mais ne cherchez pas à lui 
en substituer un autre, sous prétexte que celui-ci réussit en une autre contrée.

$3^{\circ} \mathrm{N}^{\prime}$ écontez pas les dires du public, ni les conseils des soi-disant praticiens, experts en matière de forêts. Étudiez vous même les questions et n'agissez qu'après étude faite ou conseils demandés à des hommes véritablement instruits. On ne saurait croire jusqu'où va l'ignorance du public en matière de forêts. Ici, on vous donnera le conseil de réaliser le matériel de la forêt que vous venez d'acheter et même de l'exploiter à blanc estoc. Qu'un marchand de bois agisse ainsi, on le conçoit; en achetant une forêt, il n'a pas fait autre chose qu'acquérir la matière de son commerce. Mais qu'un propriétaire consacre $100,000 \mathrm{fr}$. à l'acquisition d'une forêt pour les en retirer le lendemain, avec bien du mal, avec toutes les peines et les mauvaises chances d'une exploitation à laquelle il n'est pas préparé par des relations commerciales antérieures, qu'il agisse ainsi pour n'avoir plus, en résultat final, qu'un immeuble dénudé et improductif, cela est-il raisonnable? Là, les gens de la campagne, vos gardes même vous diront : votre taillis dépérit; il est temps de le couper ! Ces personnes, ne voyant que le petit côté des choses, sont frappées de ce que, à un certain âge, vers 12 ou 15 ans, les coudriers, morts bois et essences secondaires périssent par défaut de lumière et jonchent le sol de leurs débris. Ils ne voient pas que la place de ces brins sans valeur est occupée par des tiges d'essences dures, chênes, hêtres, charmes, que les débris des autres sont des éléments de restitution au sol appauvri et que le peuplement, loin de dépérir, commence seulement à prendre une valeur réelle. Ailleurs, on ne manquera pas de vous dire : tel gros arbre ne gagne plus, il est temps de le couper ! Un arbre gagne toujours, puisque l'enveloppe de bois annuellement fabriquée, quoique de plus en plus mince, recouvre chaque année une surface de plus en plus grande. 
N'abattez jamais un arbre qu'après avoir bien calculé ce qu'il gagne à rester sur pied et ce que vaudront les tiges nées en remplacement.

Certains propriétaires nettoient leurs forêts, détrappent leurs taillis, coupent systématiquement tout ce qui est brin traînant ou remplissage dans la forêt pour lui donner cle l'air et en tenir le sol net partout. Cette pratique est des plus mauraises; l'air ne manque jamais à la forêt ; on dessèche le sol, on diminue sa fertilité en lui enlevant les éléments les plus notables de sa restitution, et on dépense stérilement son argent à recueillir des produits sans valeur. On ne doit jamais nettoyer, c'est-à-dire faire le sol net, que sur la coupe principale, sur celle qui est destinée à la régénération d'une forêt de taillis ou de futaie pleine $\left({ }^{1}\right)$.

D'autres ont toujours la hache ou la serpe à la main, taillant les arbres pour arrondir la forme de leurs cimes, les élaguant pour élever leur fût, poursuivant les chicots et les branches mortes, allant jusqu'à panser au coaltar les plaies qu'ils font à leurs arbres. Toute la peine qu'ils se donnent n'aboutit qu'à une mauvaise opération et à des dépenses onéreuses. L'exploitation des forêts n'est pas de l'arboriculture; la taille des arbres ne fait que tourmenter les formes naturelles de cimes spéciales à chaque essence, et amoindrir la fabrication du bois en diminuant le développement foliacé. Les élagages n'aboutissent qu'à garnir les fûts de plaies ou de solutions de continuité qui en diminuent la valeur $\left({ }^{2}\right)$. Il ne faut jamais oublier que la

(1) Il ne faut pas confondre les nettoiements ainsi entendus, faisant le sol net dans toute l'étendue d'une forèt, avec les nettoiements qu'on doil consciller daus les jeunes recrus de taillis ou dans les jeunes semis de futaies; ceux-ci ont pour but de dégager uniquement les jeunes cimes des essences d'élite, telles que le chêne, pour leur donner de la lumière et les empêcher de périr étoutfẻes au milieu des essences de végétation plus rapide qui les entourent.

(2) Il faut distinguer dans ces opérations la taille, qui a pour but de façon- 
forêt est un bien naturel, fonctionnant seul sous la simple surveillance, voire même sous la direction de son propriétaire, et que la dépense faite dans le but prétendu d'activer ses forces de production n'est, trop souvent, que peine perdue et argent mal employé quand le propriétaire n'a pas fait une étude spéciale et approfondie de la sylviculture.

$\mathrm{N}^{\circ}$ 15. - On a souvent dit qu'il est plus difficile de conserver un patrimoine que de l'acquérir. Ce proverbe n'est pas sans vérité et sans application aux forêts. Quelques conseils à cet égard auront peut-être de l'utilité.

$1^{\circ}$ Soyez modéré dans la jouissance de votre forêt et ne lui demandez pas de trop gros revenus! Le conseil ne s'applique évidemment qu'aux forêts aménagées, c'està-dire disposées en vue d'un produit annuel et non à celles dont l'exploitation est périodique et constituée pour fournir un capital à des intervalles plus ou moins éloignés. Dans les forêts aménagées il y a toujours une certaine quantité de bois, c'est-à-dire d'arbres et de peuplements, qui reste fixe, invariable et constante et qui forme le matériel de l'exploitation ( $\left.{ }^{1}\right)$, comme les bâtiments de la ferme, les outils, le bétail forment le capital nécessaire à l'établissement d'un revenu agricole soutenu. Or dans les forêts, le matériel d'exploitation est formé par du bois, comme le revenu lui-même, il en résulte que la

ner les cimes, l'élagage, qui cnupe les branches pour augmenter le füt, et l'émondage, qui dégage le fùt de jeunes branches parasites et gourmandes nées au-dessous de la cime à la suite de l'exploitation de la coupe. L'émondage seul doit être conseillé, car sans lui les cimes dépérissent, se garnissent de bois mort et l'arbre peut fort souffrir du développement exagéré des branches gourmandes. L'émondage le plus tòt fait est le meilleur et le moins coùteux.

(1) Par exemple, dans un taillis aménagé à 30 ans, la valeur de la superficie boisée des coupes de 1 à 29 ans forme le matériel d'exploitation de la lorèt. 
confusion est toujours possible et qu'on est sans cesse exposé à prendre pour revenu quelque chose du capital, à diminuer ainsi la valeur vénale de l'immeuble. Avec de la modération dans la jouissance, on améliore au contraire d'une facon très notable cette valeur capitale, car on a vu ( $\left.n^{\circ} 6\right)$ que l'économie la plus faible, en apparence, a des effets très notables clans l'avenir. Il ne faut pas se plaindre de ce que, par suite de cette modération dans la jouissance, la forêt rapporte peu et de ce que le taux de placement s'abaisse. Le travail de l'arbre a cette propriété remarquable qu'au moment où le taux pour cent de son produit diminue, sa valeur capitale augmente. On ne saurait, vraiment, lui demander de fournir à la fois gros revenu et gros capital.

Un autre conseil est à dëduire de cette règle de modération dans la jouissance, c'est d'asseoir sur le terrain et de marquer par des laies l'aménagement qu'on aura adopté. Il y aura à cela double avantage : c'est que, d'abord, la propriété aura plus de valeur, comme cela arrive de tout ce qui est mis en bon ordre et en sage conduite. Ensuite, l'assiette de l'aménagement a pour but de déterminer le matériel d'exploitation, de telle sorte que les coupes le laissent intact et constant. On sera ainsi beaucoup moins exposé à des abus de jouissance et on pourra toujours mesurer exactement de quelle facon on se conduit.

$2^{\circ} \mathrm{N}^{\prime}$ introduisez jamais de bétail, petit ou grand, isolé ou en troupeau dans votre forêt. Celle-ci est un bien destiné à produire du bois; on ne saurait lui demander, à la fois, deux productions, l'herbe et le bois, pas plus qu'on ne saurait exiger deux moutures du même sac de blé. Le bétail a une action lente, mais sûrement et fatalement désastreuse pour la conservation de l'état boisé. Elle se manifeste par' le piétinement qui durcit le sol et lui enlève ses qualités physiques - par la destruction de la couverture végétale 
qui ôte au terrain les éléments chimiques de sa reconstitution, - par l'abroutissement des bourgeons, des semis et des pousses, anéantissement de tout ce qui est jeune et tendre dans la forêt, enlevant le bien présent et jusqu'aux espérances de l'avenir. Dès le début, le mal paraít peu, mais les places vides ne tardent pas à se montrer, les clairières s'agrandissent, la végétation languit, la ruine devient menacante.

$3^{\circ}$ Ne défrichez jamais votre forêt; tout défrichement est une destruction de capital créé, de richesse acquise, pour lui substituer un capital à créer, une richesse incertaine. A quoi bon transformer un bon et solide matériel-bois destiné à l'exploitation d'une forêt, en un autre matériel de bâtiments, de bétail et d'instruments agricoles destiné à l'exploitation d'une ferme? Est-ce pour profiter de la différence de valeur? Mais, outre qu'elle est en général peu sensible, mieux vaudrait, si vous avez un besoin pressant d'argent, faire un emprunt à votre forét, sauf à la reconstituer peu à peu dans l'avenir, que changer le mode d'exploitation de votre terre. Au lieu d'un bien naturel travaillant seul et sans aucun souci de votre part, vous allez vous trouver en face d'une exploitation compliquée, qui exigera tous vos soins et peut-être tout votre travail. Si c'est pour vendre la terre ainsi défrichée que vous faites cette opération, songez qu'en France, c'est bien plutôt le cultivateur qui manque à la terre que la terre à la culture et que de ce côté vous pouvez avoir de gros mécomptes.

L'expérience en a été faite: les sols forestiers ne sont pas constitués comme les terrains agricoles. Dans ceux-ci, la couche arable est ameublie par un labour incessant, enrichie par un travail fort ancien, par des engrais réitérés ; le sol doit toute sa valeur à la richesse de cette couche. Dans les terrains forestiers, la puissance productive est due à un ameublissement causé par un couvert prolongé, 
à la présence des racines, des graines, des feuilles mortes, d'humus et de terreau, à tout un ensemble encore assez indéterminé, mais bien certain et que les cultivateurs désignent très nettement en distinguant les sols forestiers sous le nom de terreà bois. Les conditions ne sont pas les mêmes et expliquent comment il est parfois si difficile et toujours si long de reboiser un champ cultivé et de le transformer en véritable forêt. L'inverse se produit lorsqu'on consacre à la culture agricole un sol forestier défriché. Pendant les premières années tout va bien et les récoltes sont fort belles, mais ce n'est qu'un feu de paille et bientôt il faut s'appliquer à constituer ce sol agricole qui n'a été acquis dans nos campagnes que par les longs efforts de nombreuses générations. Ajoutez à cela qu'une forêt défrichée se prête le plus souvent fort mal à la constitution d'une ferme, c'est-à-dire à un ensemble d'exploitation agricole. Le sol y est à peu près partout de même nature, il fournit peu ou presque rien à la création des prairies naturelles, encore moins à l'établissement des cultures industrielles qui seules font l'aisance de l'agriculteur. Il n'y a donc rien d'étonnant à ce que maintenant on trouve à acheter pour de misérables sommes des étendues considérables de terrains jadis défrichés, et à ce que l'on reboise actuellement des sols où des destructeurs de forêts se sont ruinés par ignorance ou cupidité.

No16. - Dans les conseils précédents, nous nous sommes adressés aux particuliers propriétaires de hois. Estce à dire que les Agents de l'administration des forêts doivent les dédaigner et n'en tenir aucun compte? Ne sontils pas tenus à une instruction scientifique et professionnelle plus grande encore, eux qui sont appelés à éclairer les particuliers et à renseigner les tribunaux comme experts des litiges forestiers? Ne sont-ils pas les mandataires obligatoires, les administrateurs légalement imposés des 
communes et des établissements publics propriétaires de bois? Combien doivent-ils à ce titre, se défendre contre les fantaisies dans les modes de traitement, conserver la forêt contre les jouissances du présent, être instruits, savants même, pour éclairer les communes sur leurs véritables intérêts, discuter âvec elles les questions techniques et les leur faire bien comprendre.

Cette mission d'extrême confiance que la loi donne aux Agents forestiers dans les forêts des communes leur impose le devoir d'une instruction étendue et justifierait à elle seule l'établissement de l'École nationale forestière, si leur fonction était à ce point réduite; mais les autres services qu'ils ont à rendre au public par la gestion du domaine forestier de l'État, par la grande et belle œuvre de la restauration des montagnes, par leur délicate action dans l'agriculture pastorale de France exig'ent d'eux de nouveaux efforts et une instruction supérieure.

Parmi ces utiles et hautes fonctions du corps forestier, nous en retiendrons une, parce qu'elle se rapporte à notre sujet d'études et qu'elle est, en quelque sorte, plus élevée encore. Les Agents forestiers sont les conservateurs des ressources en bois indispensables à notre travail national, gardiens de l'épargne passée, producteurs du travail futur, hommes de haute mission, car ils ont charge de défendre le pays contre les périls de l'avenir, et la disette la plus dangereuse, la disette du travail.

A eux surtout, à eux plus encore qu'aux particuliers propriétaires de bois, nous disons sans nous lasser :

Fattes des ARbres! 


\section{CHAPITRE II}

\section{THÉORIE DE L'INTÉRÊT}

ArTicle $1 \mathrm{er}$.

\section{Génẻralitẻs.}

Nos 17. - Loyer des capitaux ou intérèt.

18. - Trois lois de formation des capitaux.

19. - Deux questions fondamentales dans chaque loi de formation : 10 relation entre le capital initial et le capital futur; 20 relation entre le capital initial et son loyer : quatre problèmes et quatre tarifs.

20. - Notations adoptées.

$N^{\circ} 17 .-$ Le propriétaire qui affecte une partie de sa fortune à planter des bois ou à acheter une forêt est obligé, comme tous ceux qui se livrent à une entreprise agricole ou industrielle, de comparer à chaque instant la manière dont la richesse se forme dans sa forêt avec celle qui régit la constitution des capitaux dans leur emploi habituel. Cette comparaison ne peut s'effectuer que par l'exacte connaissance de la théorie de l'intérêt, puisque le temps est le facteur principal de la formation de la valeur dans les forêts.

Cette théorie de l'intérêt est trop souvent ignorée des propriétaires de forêts, parce qu'elle se présente dans leurs souvenirs classiques comme hérissée de difficultés. Elle est simple cependant, et nous allons essayer de l'exposer avec les procédés de calculs les plus élémentaires.

On n'est véritablement producteur et fabricant de bois 
qu'à la condition de se rendre compte des conditions dans lesquelles on produit la valeur pécuniaire et on constitue le capital de sa fortune.

Le capital est le produit de l'épargne: c'est du travail accumulé, mis en réserve pour les besoins de la vie. A ce titre, il a droit à une rémunération quand son propriétaire le préte ou le loue à ceux qui en font emploi, l'utilisent dans une entreprise ou en ont besoin pour vivre.

Cette rémunération, qui s'appelle en général toyer", prend le nom particulier d'intérêt quand il s'agit d'une forme spéciale du capital : l'or ou l'argent monnayé. Ce nom spécial d'intérêt pour le loyer du capital argent a son origine dans une législation ancienne, anti-économique, dont notre époque positive et libérale ne s'est pas encore totalement affranchie, car le loyer de l'argent n'est pas libre et les conventions sont limitées par un maximum, que les parties ne peuvent dépasser. (Lois du 3 septembre 1807 et du 12 janvier 1886.)

Il y a une mesure à l'intérêt, comme il en est à toutes les choses qu'on veut évaluer. Cette mesure qui est, naturellement, une fraction du capital prêté, est maintenant'il n'en a pas toujours été ainsi) une fraction centésimale du capital ; l'intérêt est un, deux, trois, etc., centièmes du capital, selon les conditions où celui-ci s'est loué. Le taux est le numérateur de cette fraction, il est la mesure du loyer; c'est donc l'intérêt de cent francs pendant un an.

Ce dénominateur constant permet de simplifier les calculs bien plus que si on prenait 1/20 pour mesure au lieu de 5 pour cent ou 3/50 au lieu de 6 pour cent.

Pour simplifier encore davantage et pour ne pas traîner dans les calculs le dénominateur cent, on raisonne sur le taux par rapport à 1 franc (dans nos notations $t$ sera l'intérêt de 1 franc, 0,05 centimes, à 5 pour cent par exemple).

$\mathrm{N}^{\circ}$ 18. - Pour former le capital, l'épargne peut s'accu- 
muler de plusieurs façons : $1^{\circ}$ d'une manière irrégulière ou sans règle déterminée; $2^{\circ}$ d'une manière régulière, c'est-à-dire suivant des lois constantes. Dans ce dernier cas, seulement, la formation du capital peut s'exprimer par des formules mathématiques donnant matière aux recherches de l'esprit.

Il y a trois manières régulières, trois lois mathématiques de la formation des capitaux:

Celle des intérêts composés;

Celle des annuités;

Calle des annuités périodiques.

Si on convient que le loyer d'un capital initial s'ajoutera à ce capital pour produire lui-même intérêt pendant un temps déterminé, on dit que le capital futur aura été formé par l'adjonction de l'intérêt des intérêts ou par l'intérêt composé.

Lorsque, au contraire, le capital est formé de sommes égales qui s'ajoutent chaque année avec la condition que les intérêts des intérêts s'y réuniront eux-mêmes, on a la loi de formation par annuités.

Si, enfin, la somme égale qui forme le capital ne se produit pas chaque année, mais à des intervalles égaux formés d'un certain nombre d'années, en y ajoutant toujour's l'intérêt composé des sommes accumulées, le capital est créé suivant la loi des annuités périodiques $\left({ }^{1}\right)$.

(1) Nous arons omis à dessein de parler d'une loi de formation, dite des intéréts simples, qui n'est qu'une conception mathématique purement spéeulative et sans application pratique. Peut-on conceroir, en effet, qu'un individu ajoutera tous les ans, au capital initial, l'intérêt qu'il a produit sans demander un loyer à celte somme dont il se prive ainsi sans rien en retirer? Cette singulière conception permet d'établir par analogie des formules correspondantes à celles de l'intérêt composé : capital futur, capital actuel, facteur générateur; mais alors les résultats qu'elles donnent se conçoirent encore bien moins que la loi de formation qui leur a donné naissance et n'ont plus, en réalité, aucune signification.

Un ancien auteur allemand, Cotta, a mème imaginé une loi intermédiaire de formation, dite de l'intérét moyen, et qui est la moyenne arithmétique 
Les épargnes accumulées par la forêt ne suivent aucune de ces trois lois mathématiques de formation; elles s'ajoutent suivant une loi qui leur est spéciale et qui est encore inconnue; mais il est juste de reconnaître qu'il y a des analogies frappantes entre la manière dont la valeur est formée dans les forêts et la façon dont elle se constitue par le placement des capitaux.

Ainsi dans un massif boisé, le bois produit par une année s'ajoute à la masse ligneuse pour produire luimême du bois, c'est une formation analogue à celle de l'intérêt composé.

Ainsi encore, la production annuelle d'un hectare de forêt peut être considérée comme une quantité constante (production) qui s'ajoute à elle-même chaque année, mais en prenant une valeur pécuniaire de plus en plus grande, de sorte qu'elle forme le capital d'une façon analogue à la loi des annuités.

Enfin certains produits des forêts (fainées, glandées, résines, émondages) fournissent des sommes sensiblement égales, qui se reproduisent à des intervalles périodiques. On peut considérer la valeur en provenant comme formée par la loi des annuités périodiques.

Mais ce ne sont que des analogies, car la végétation a sa loi propre qui n'est pas celle des intérêts composés, ni celle des annuités, ni celle des annuités périodiques. Certains auteurs forestiers ont été souvent entraînés à confondre l'analogie avec l'identité et à en déduire des méthodes d'estimation ou d'investigation délicates et grosses d'erreurs. Il est donc indispensable de connaître à fond la théorie de l'intérêt pour appréciel sainement ces méthodes et en reconnaître l'exactitude ou l'imperfection.

entre la loi de l'intérêt simple et celle de l'intérêt composé. Que dire d'une loi qui est ainsi le résultat de la combinaison d'une erreur arec une rérité ? Cotta n'a jamais prouvé d'ailleurs que la régétation forestière suivait dans sa marche cette singulière loi de l'intérêt moyen (voir Note A). 
D'un autre côté, il est certain que si la forêt ne se forme pas suivant ces lois mathématiques de l'intérêt, la valeur qu'elle produit et le résultat final peuvent très bien et dans de nombreuses circonstances de la vie être comparés avec la valeur qui serait produite par des placements d'argent; il y a donc utilité pour le forestier à comparer les résultats de ces placements et de leurs combinaisons financières avec les produits de la forêt. Il faut, dès lors, qu'il connaisse tous les problèmes de l'intérêt.

$\mathrm{N}^{\circ}$ 19. - Nous aurons donc, dans ce double but, à étudier pour chacune de ces trois lois de formation des capitaux, les différents problèmes qui en naissent et qui sont au nombre de deux principaux :

$1^{\circ}$ Calculer la valeur future $\mathrm{M}$ d'un capital initial $\mathrm{P}$ d'après la loi de sa formation, par exemple celle des intérêts composés, et pour un temps limité $n$. C'est la relation entre $\mathrm{M}$ et $\mathrm{P}$.

$2^{\circ}$ Calculer le loyer, l'intérêt $\mathrm{R}$, produit pendant un temps limité $n$, par un capital primitif $\mathrm{P}$, en supposant qu'on ait suivi la même loi de formation. C'est la relation entre $\mathrm{P}$ et $\mathrm{R}$.

Ces deux problèmes principaux fournissent les relations qui existent : $1^{\circ} l^{\prime}$ une entre le capital initial ou actuel et le capital futur ; $2^{\circ}$ l'autre entre le capital primitif et son loyer.

Il en résulte deux autres problèmes qui ont pour but tous deux, d'exprimer le capital initial.

Le premier donne le capital primitif $\mathrm{P}$ en fonction du capital futur $\mathrm{M}$, c'est la valeur actuelle d'un capital à échéance future.

Le second donne le capital primitif $\mathrm{P}$ en fonction $d u$ loyer ou de l'intérêt $\mathrm{R}$ sans avoir besoin de connaître par conséquent le capital futur formé par l'adjonction du capital primitif au loyer. Comme ce loyer est susceptible de 
se reproduire indéfniment à chaque même intervalle de temps, il en résulte que l'expression de ce capital initial a un autre caractère : elle n'est plus la valeur actuelle d'un capital futur qui sera dans l'avenir constituépar un temps limité; elle est la valeur même d'un loyer qui se reproduit à des intervalles égaux, plus ou moins éloignés, mais d'une manière perpétuelle. Ce capital primitif doit être considéré, alors, comme le fonds générateur d'une rente $\mathrm{R}$ périodique et perpétuelle. Pour mieux marquer une distinction sur laquelle nous aurons l'occasion de revenir, nous le désignerons par la lettre $\mathrm{F}$.

Nous aurons ainsi quatre problèmes qui consisteront à chercher :

$1^{\circ}$ Le capital futur à l'aide du capital initial ;

$2^{\circ}$ Le loyer de ce capital primitif ;

$3^{\circ}$ Le capital initial d'après le capital futur;

$4^{\circ}$ Le capital générateur d'après son loyer.

On est fondé à en déduire qu'il faudra quatre tarifs ou comptes-faits, établis à différents taux, pour faciliter les calculs. Nous verrons qu'ils se réduisent, en réalité, à trois par une légère modification à apporter au premier.

$\mathrm{N}^{\circ}$ 20. - Pour la solution de ces quatre problèmes qui consistent à trouver :

M valeur future d'un capital,

$\mathrm{R}$ intérêt de ce capital,

$\mathrm{P}$ valeur du capital primitif en fonction du capital futur,

$F$ valeur du capital initial en fonction de son intérêt,

nous nous serrirons des notations suivantes pour exprimer les termes en usage dans les études de cette nature :

$\mathrm{R}$ revenu, intérêt, loyer du capital;

$t$ taux de l'intérêt pour 1 franc, intérêt de 1 franc pendant un an; 
M valeur future du capital; montant du capital grossi de son intérêt ou d'un certain nombre d'économies annuelles égales; montant de ces annuités;

P la valeur actuelle du capital; valeur des le principe; principal d'un certain nombre d'annuités ;

$\mathrm{F}$ fonds générateur du lojer; valeur productrice de l'intérêt ou d'une rente périodique perpétuelle ;

a annuité, économie annuelle ou périodique;

$n$ temps, nombre d'années penclant lequel le capital fonctionne et se forme (ce n'est pas l'année de la jouissance, c'est le nombre d'années du fonctionnement);

$p$ périodicité des annuités, intervalle de temps d'une annuité à l'autre, nombre des années qui les séparent:

$s$ suspension dans le fonctionnement du capital, nombre d'années pendant lequel le capital est grevé d'usufruit et pendant lesquelles on n'en a que la nue propriété :

$u$ usufruit, jouissance limitée dans sa durée, d'une rente annuelle ou d'une rente périodique.

Il ne sera jamais utile dans les questions forestières de considérer des fractions d'années et $n$ sera toujours un nombre entier.

Faisons remarquer, en passant, qu'une singulière disposition de notre loi civile interdit la composition de l'intérêt par fractions d'années (art. 1154) et introduit la défense légale dans une matière où l'ordre public n'a rien à voir et où la liberté des conventions devrait être souveraine. Le législateur de 1804 aurait été bien étonné si on lui arait prédit qu'un jour viendrait où la composition des intérêts par semestres serait un des plus féconds instruments de crédit pour les compagnies de chemins de fer par l'ingéniellx mécanisme de leurs obligations et un puissant moyen de libération des héritages entre les mains des institutions financières de prêts fonciers. 
Article 2.

\section{Loi de formation par l'intérêt composé.}

Nos 21. - Premier probleme. - Capital futur M en fonction du capital initial P.

22. - Deuxième problème. - Revenu on intérèt $R$ en fonction du capital initial $\mathrm{P}$.

23. - Tarif I, facteur de sommation et facteur d'intérêt.

24. - Troisieme problème. - Valeur actuelle P d'un capital futur M en fonction de ce capital.

25. - Tarif II, facteur de réduction à l'actualité. - Escompte.

26. - Quatrième problème. - Valeur du capital générateur $\mathbf{F}$ en fonction du revenu $\mathbf{R}$.

27. - Tarif III, lacteurs on deniers de génération d'un revenu périodique.

28. - Observations sur l'emploi de cette formule.

29. - Problèmes dérivés. - Trois problèmes déduits de la relation entre $\mathbf{P}$ (capital initial) et $\mathrm{M}$ (capital futur).

30. - Trois problèmes déduits de la relation entre $\mathbf{F}$ (fonds générateur) et $\mathrm{R}$ (intérêt produit).

3I. - Observations sur la valeur du capital initial et du fonds générateur.

32. - Résumé des intérêts composés.

$\mathrm{N}^{\circ}$ 21. - Premier problème. - La valeur future $\mathrm{M}$ d'un capital originaire $\mathrm{P}$ grossi de ses intérêts composés pendant $n$ ans au taux $t$ est :

$$
\mathrm{M}=\mathrm{P}(1+t)^{n}
$$

En effet, au bout de 1 an, un capital de 1 fr. devient $1+t$, celui de $\mathrm{P}$ fr. devient $\mathrm{P}(1+t)$. Au bout de 2 ans, ce capital devient $\mathrm{P}(1+t)(1+t)=\mathrm{P}(1+t)^{2} \ldots$. et au bout de $n$ ans, il devient $\mathrm{P}(1+t)^{n}$.

Cette formule se résout par les logarithmes de la manière suivante :

$$
\text { Log. } \mathrm{M}=\log \cdot \mathrm{P}+n \log \cdot(1+t)
$$

Il est souvent plus expéditif de se servir d'une table (tarif I) qui donne les puissances successives de $(1+t)$ 
pour les différents taux usuels de $1 / 2$ pour cent à 5 pour cent $\left({ }^{1}\right)$.

Exemple: On dépose 400 francs à la caisse d'épargne postale à $30 / 0$, on l'y oublie pendant 35 ans : quelle somme aurat-on à cette époque?

$$
400 \times 2,813=1,125 \mathrm{fr} .
$$

Celui qui achèterait 400 francs un hectare de taillis rasé avec la certitude de le vendre $1,125 \mathrm{fr}$. dans 35 ans aurait aussi une caisse d'épargne dont le résultat final serait, comme celui de la caisse d'épargne postale, dı $30 / 0$ à intérêts composés. Mais cela ne veut pas dire que, pendant la durée des 35 ans, la valeur de l'hectare aura progressé identiquement et à chaque année comme les 400 francs déposés à la caisse d'épargne, c'est-à-dire suivant la loi de l'intérêt composé à $30 / 0$; les valeurs progressires de la forêt ont une marche toute différente.

No 22. - Deuxième problème. - On déduit de la formule précédente (1) que l'intérêt $\mathrm{R}$ produit par le capital P placé pendant $n$ ans à intérêts composés au taux $t$ s'obtient naturellement en retranchant $P$.

$$
\mathrm{R}=\mathrm{P}\left[(1+t)^{n}-1\right]
$$

Cette expression n'est pas logarithmique, mais on la calcule très facilement à l'aide des facteurs du tarif I dont on retranche l'unité.

Dans l'exemple précédent, l'hectare nu est un capital initial qui vaut 400 francs, l'intérêt de cette somme déposée à la caisse d'épargue pendant 35 ans sera bien à $30 / 0$ :

$$
400 \times 1,813=725 \mathrm{fr} \text {. }
$$

Mais le taillis de 35 ans vaudra-t-il exactement 725 francs? Il n'aura cette valeur vénale, absolue, que si le sol vaut bien 400 francs et si la loi de croissance est identique à celle des intérèts composés à $30 / 0$, car ce sont les deux facteur's de 725 francs.

( ${ }^{1}$ ) Nous arons publié ce tarif 1 pour 10 taux de $1 / 2$ à 5 pour cent dans nos Estimations concernant la propriété forestière, 1 vol. gr. in-8o, Paris, Marchal et Billard, 1886. 
$\mathrm{N}^{\circ}$ 23. - Le tarif I est construit pour un capital primitif de 1 franc; il contient, en réalité, deux tarifs, puisqu'il fournit: $1^{\circ}$ le capital augmenté de ses intérêts; $2^{\circ}$ les intérêts de ce capital; ce dernier résultat est obtenu en retranchant l'unité du facteur, c'est-à-dire le capital initial, 1 franc. La formation du tarif pour 1 franc de capital fait que, dans ces deux problèmes ainsi ramenés à l'unité, les résultats perdent leur signification relative pour en prendre une absolue qui est celle d'un facteur abstrait de capitalisation ou plutôt de sommation des intérêts, ou celle d'un facteur d'intérêt quand on en retranche l'unité.

Nous avons emprunté, en les complétant, les tarifs I, II et III aux tables publiées, en 1876, à Leipzig,par M.Gust. Heyer, directeur de l'École forestière de Munden.Des tables de cette nature, mais réduites aux taux de 3 à 5 pour cent, ont été originairement publiées, en 1827, par H. Cotta, directeur de l'École forestière de Tharand (Saxe). Elles ont été introduites en France par M. de Salomon, en 1837, el reproduites par M. Nanquette en 1859. (Voir nos Estimations concernant la propriété forestière).

$\mathrm{N}^{\circ}$ 24. - Troisième problème. - Voici une conséquence du premier problème (relation entre la valeur initiale $\mathrm{P}$ et la valeur future $\mathrm{M}$ ). On veut connaître la valeur actuelle d'un capital qui ne sera constitué que dans $n$ ans. La formule (1) donne

$$
\mathrm{P}=\frac{\mathrm{M}}{(1+t)^{n}}
$$

C'est la valeur de M ramenée à l'actuatité; cette valeur actuelle de $\mathrm{M}$ peut être très petite quand $n$ augmente, c'est-à-dire à mesure que l'échéance s'éloigne, mais elle est toujours positive.

Cette formule se résout par les logarithmes de la manière suivante :

$$
\text { Log. } \mathrm{P}=\log . \mathrm{MI}-n \log \cdot(1+t)
$$


Elle se résout plus facilement par letarif II qui contient les inverses des nombres du tarif I.

Exemple : un billet de 1,125 fr. est à échẻance de 35 ans; quelle est sa valeur actuelle à $30 / 0$ ?

$$
1125 \times 0,355=400 \mathrm{fr} \text {. (tarif II) }
$$

Un taillis exploité actuellement se vendra très probablement 1,125 fr. (terrain 400 et bois 725) dans 35 ans. On voit par le calcul du billet que ce bois, s'il pousse suivant sa loi propre, fournit une valeur finale comme le ferait un capital placé à $30 / 0$. Quelle est la valeur actuelle, vénale de la propriété nue? Est-ce $400 \mathrm{fr}$. ? non! car la valeur vénale de la propriẻté peut être tout autre et dépend des ventes faites dans la localité. Mais il est certain que cette propriété vaudra exactement $400 \mathrm{fr}$. pour celui qui voudrait faire emploi de ses fonds à $30 / 0$ peudant 35 ans seulement.

Examinons maintenant le taillis : s̈il vaut $725 \mathrm{fr}$. à 35 ans, sa valeur à 1 an est celle de 257 fr. !

$$
725 \times 0,355=257 \mathrm{fr} \text {. (tarif II) }
$$

Est-ce la valeul' vénale de ce taillis de 1 an ?

Non encore : car personne ne songera à payer ou à estimer $257 \mathrm{fr}$. une pousse de 1 an. Mais il n'en est pas moins vrai de dire que si un acheteur de récoltes rencontrait un propriétaire vendeur à $257 \mathrm{fr}$. de cette seule pousse de 1 an (le vendeur conservant la proprièté du terrain), l'acheteur aurait placé son argent pendant 35 ans à $30 / 0$.

On voit de quelle manière il taut entendre les choses.

No25. - Le tarif II donne les valeurs actuelles de 1 franc payable au bout d'un certain nombre d'années. Ainsi réduits à l'unité, les résultats deviennent des facteurs de valeurs actuelles ou de réduction d'un capital à l'actualité. Ce sont les inverses des facteur's du tarif' I. Le tarif II suppose l'achat d'une valeur limitée quant à su durée, et dont on devient propriétaire pour un temps donné. C'est le cas de l'escompteur d'un billet à échéance fixe ou de l'acheteur d'une coupe à réaliser dans un certain temps, le vendeur restant propriétaire de la forêt. (Voir nos Estimations concernant la propriété forestière.) La formule (3) qui sert de base au tarif II, donnant la 
valeur actuelle d'un billet à échéance ultérieure, on en déduit que l'escompte $\mathrm{E}$, c'est-à-dire la somme qu'on retient sur le billet pour l'acheter ou le payer immédiatement, est le montant diminué de sa valeur actuelle.

$$
\mathrm{E}=\mathrm{M}\left(1-\frac{1}{(1+t)^{n}}\right)=\mathrm{M} \frac{(1+t)^{n}-1}{(1+t)^{n}}
$$

(employer le facteur du tarif I, le diminuer d'une unité et multiplier par le facteur du tarif II)

C'est l'escompte naturel, ou l'escompte allemand, ou en dedans. L'escompte français ou en dehors est le montant du billet diminué de son intérêt pendant $n$ ans.

$$
\mathrm{E}=\mathrm{M}\left[1-(1+t)^{n}+1\right]=\mathrm{M}\left[2-(1+t)^{n}\right]
$$

quantité qui devient vite négative. Dans ce système d'escompte, un billet de 1,000 francs à échéance de 15 ans n'aurait point de valeur à 5 pour 100 puisque $\overline{1.05}^{15}=2.079$.

Cela montre que l'escompte en dedans est seul logique et naturel, car ce billet ne saurait être dépourvu d'une valeur qui est, à 5 p. 100 , de 481 francs.

Escompte : $1000 \times 1,079 \times 0,481=519 \mathrm{fr}$.

Valeur : $1000-519=481 \mathrm{fr}$.

ou : $1000 \times 0,481=481 \mathrm{fr}$.

(tarif' $I I)$

Au surplus, dans un cas pareil, le banquier escompteur abaisserait le taux qui sert de mesure à son achat, c'est affaire de convention et de langage commercial.

$\mathrm{N}^{\circ}$ 26. - Quatrième problème. - Connaissant le loyer, l'intérêt produit $\mathrm{R}$ par un certain capital initial $\mathrm{P}$ placé à intérêts composés au taux $t$ pendant $n$ ans, calculer ce capital primitif en fonction de R.

De la formule (2)

$$
\mathrm{R}=\mathrm{P}\left[(1+t)^{n}-1\right]
$$

qui établit la relation entre $\mathrm{R}$ et $\mathrm{P}$, on déduit

$$
\mathrm{P}=\frac{\mathrm{R}}{(1+t)^{n}-1}
$$


Comme l'intérêt $\mathrm{R}$ est susceptible de se reproduire indéfiniment tous les $n$ ans, on doit considérer le capital primitif $\mathrm{P}$ comme le fonds générateur d'une rente périodique ou d'un revenu $\mathrm{R}$ se produisant indéfiniment tous les $n$ ans.

L'acquéreur au prix déterminé par cette formule devient propriétaire à tout jamais d'un fonds indéfiniment producteur de R. Pour indiquer cette différence nous écrivons cette expression $\left({ }^{1}\right)$ :

$$
\mathrm{F}=\frac{\mathrm{R}}{(1+t)^{n}-1}
$$

Cette formule ne se résout pas par les logarithmes; on l'obtient très facilement par le tarif III qui donne le capital générateur d'une rente périodique de 1 franc.

Exemple: On veut acheter à $30 / 0$ un revenu de $725 \mathrm{fr}$. qui se reproduira tous les 35 ans, quel prix faut-il en donner?

$$
725 \times 0,551=400
$$

Faut-il en déduire que le sol (garni de son ensouchement) d'un taillis qui rapporte 725 fr. tous les 35 ans vaut $400 \mathrm{fr}$.? Oui, s'il est constaté qu'on cherche une valeur non courante et marchande, mais simplement conventionnelle et relative à un acheteur qui veut faire emploi de son argent à $30 / 0$, mais non, s'il en est autrement.

Voici, pour mieux le faire comprendre, un hectare, tout voisin du taillis; il est planté en pins de 100 ans dont la coupe vaut $4,000 \mathrm{fr}$. Assurément, le sol se vendrait $400 \mathrm{fr}$. comme celui du taillis; or, le capital générateur à $30 / 0$ tous les 100 ans est de $220 \mathrm{fr}$.

$$
4000 \times 0,055=220
$$

Pourquoi ce résultat si différent? C'est que pour la coupe des pins à 100 ans valant $4,000 \mathrm{fr}$., la végétation n'a pas mar'ché de 1 à 100 ans comme un capital placé à intérèts composés à $30 / 0$; c'est avec une vitesse beaucoup moindre (2 1/2 0/0 environ) qu'elle a progressé.

$$
4000 \times 0,0924=370 \mathrm{fr} . \quad(\text { tarif II) }
$$

Si l'acquéreur veut lutter avec les marcliands de bois et

( ${ }^{1}$ En d'autres termes, F n'est égal à $\mathbf{P}$ que s'il est déduit dı mème taux $\ell$ qui a servi à former $I l$ en fonction de $P$. 
avec les spéculateurs en terres, il sera obligé de faire son calcul sur un taux moindre et de se contenter d'un placement à environ $21 / 20 / 0$; autrement, s'il continuait à ne raisonner que sur un taux uniforme et moyen de $30 / 0$, il donnerait à la terre une valeur trop faible ( $220 \mathrm{fr}$.), on serait obligé, pour arriver au cours de $400 \mathrm{fr}$. l'hectare, d'assigner au revenu une valeur $(\mathbf{7}, 272$ fr. $)$ exagérée et bien supérieure à la valeur r'éelle.

$7272 \mathrm{fr} . \times 0,055=400 \mathrm{fr}$.

(tarif III)

On voit donc que si on veut estimer des sols de forêts avec le procédé des intérêts composés, il faudra les évaluer non sur un taux moyen des placements en immeubles dans la localité, mais sur un taux particulier aux forêts et spécial à chaque âge d'exploitation dans la région; autrement, le calcul par cette formule ne donne qu'une valeur conventionnelle et relative au propriétaire qui veut faire emploi de ses fonds à un taux déterminé.

$\mathrm{N}^{\circ}$ 27. - Le tarif III donne les valeurs du capital générateur d'un revenu de 1 fr. Réduits ainsi à l'unité, les résultats perdent leur valeur relative pour se transformer en facteurs absolus de génération d'un revenu périodique. Quand la périodicité est réduite à un an, le facteur générateur du capital prend le nom particulier de denier de capitalisation. Dans le placement à 5 pour cent, l'intérèt est $\frac{5}{100}$ ou $\frac{1}{20}$ du capital ; 20 est le nombre abstrait par lequel il faut multiplier le revenu pour avoir le capital. Les facteurs du tarif III sont donc des deniers de capitalisation étendus à tous les intervalles de temps où se produit le revenu.

Le tarif III est une table de facteurs donnant le prix au moyen duquel on acquiert la propriété perpétuelle d'un revenu périodique qui se reproduit sans limitation de durée quand on veut faire emploi de son argent à un taux déterminé. (Voir ce tarif III calculé pour 10 taux de $1 / 2$ ¿̀ 50/0 dans nos Estimations concemantla propriété forestière.) 
$\mathrm{N}^{\circ}$ 28. - Dans l'emploi de ce tarif $I I I$, il faut faire attention que le premier revenu $R$ ne se touche pas au début de la période, mais bien à la fin de cette période. Le capital fonctionne pendant $n$ années sans produire, c'est pour cela que cette formule a été employée par les estimations du sol forestier supposé exploité.

Si on devait toucher la première rente au début de la période, il faudrait simplement l'ajouter au résultat.

On dira, par exemple, pour un revenu de $725 \mathrm{fr}$. tous les 35 ans à $30 / 0$, que la valeur capitale est 1,125 fr.

Fonds générateur : $725 \times 0,551=\ldots \quad 400^{r}$

Premier revenu............. 725

Total........ 1125

La formule revêt, dans ce cas particulier, une autre forme qui est :

$$
\begin{aligned}
& \mathrm{F}=\mathrm{R} \frac{(1+t)^{n}}{(1+t)^{n}-1} \\
& \left(\text { tarif I } \times \text { tanif III) }^{-1}\right.
\end{aligned}
$$

Cette formule conduit au même résultat que le calcul ci-dessus, car le facteur de $R$ n'est autre que

$$
1+\frac{1}{(1+t)^{n}-1}
$$

On peut le vérifier par les tarifs $I$ et $I I I$.

$$
725 \times 2,813 \times 0,551=1,125 \mathrm{fr} \text {. }
$$

L'observation est faite pour éviter les complications, quand le revenu se perçoit, à la fois, au début et à la fin de la période (taillis non exploité mais garni de la coupe au moment de l'estimation). Elle conduira en outre, à uno formule générale qui sera la règle fondamentale des estimations relatives $\left(\mathbf{n}^{\circ} 51\right)$.

No 29. - Problèmes dérivés. -.- Les quatre problèmes fondamentaux que nous venons d'expliquer et qui correspondent aux trois tarifs précédents se rérluisent en 
réalité, à deux relations : l'une entre le capital futur et le capital primitif, l'autre entre le loyer et le capital initial.

Examinons la première de ces relations $\left(n^{\circ} 21\right)$ :

$$
\mathrm{M}=\mathrm{P}(1+t)^{n} \quad \text { (1) (tarif I) }
$$

elle donne naissance à quatre problèmes dans lesquels les quatre quantités sont successivement prises en inconnues.

Nous a vons déjà vu (n²4)

$$
\left.\mathrm{P}=\frac{\mathrm{M}}{(1+t)^{n}} \quad \text { (3) (tarif } I I\right)
$$

Il ne nous reste plus qu'à déduire le taux du placement pendant ces $n$ ans :

$$
\log \cdot(1+t)=\frac{\log \cdot \mathrm{M}-\log \cdot \mathrm{P}}{n}
$$

Ainsi que le nombre d'années pendant lesquelles le capital a été placé temporairement:

$$
n=\frac{\log \cdot M-\log \cdot P}{\log \cdot(1+t)}
$$

Voici un exemple de leur application:

On trouve en Champagne à acheter de mauvais terrains mouilleux à $100 \mathrm{fr}$. l'hectare; cultivés en aunes, dont la plantation coùte 100. ils reviennent à $200 \mathrm{fr}$. l'hectare et produisent à 20 ans $700 \mathrm{fr}$.; ils valent donc, à cet âge, au moins $900 \mathrm{fr}$.

Formule (1). - Un propriétaire possède une somme de $200 \mathrm{fr}$. et la dépose à la caisse d'épargne à $30 / 0$, quelle somme aura-t-il au bout de 20 ans ?

$$
200 \times 1,806=360 \mathrm{fr} . \quad(\text { tarif } I)
$$

ne vaut-il pas mieux ètre propriétaire d'aunaie, puisque avec une somme de $200 \mathrm{fr}$. on aura $900 \mathrm{fr}$. en 20 ans?

Formule (3). - Or, ces terrains sont recherchés; notre propriétaire reut bien les payer plus cher, mais il se demande quelle somme il ne doit pas dépasser pour qu'il ait au moins $30 / 0$ de son argent, en d'autres termes quel est le capital initial qui deviendra $900 \mathrm{fr}$. au bout de 20 ans à $30 / 0$.

$$
900 \times 0,5537=500 \mathrm{fr} . \quad(\text { tarif } I I)
$$


Comme la plantation coûte $100 \mathrm{fr}$., notre acheteur peut donc aller jusque $400 \mathrm{fr}$. l'hectare: á ce prix, il aura encore son argent capitalisé à $30 / 0$, c'est-à-dire se grossissant à intérêts composés de $30 / 0$, pendant 20 ans, seul laps de temps qu'il considère (voir $\mathrm{n}^{\circ} 31$ ) :

$$
500 \times 1,806=900 \quad(\text { tarif } I)
$$

Formule (5). - Cette plantation est très imitée dans le pays, car ce déboursé de $200 \mathrm{fr}$. devenant $900 \mathrm{fr}$. en 20 ans, correspond à un placement à intérêts composés de $80 / 0$ environ : est-ce une jolie caisse d'épargne?

$$
\begin{aligned}
& \log .900=\ldots \quad 2.95424 \\
& \log .200=\ldots \frac{2.30103}{0.65321} \\
& \log .(1+t)=\frac{0,65321}{.20}=0,03266 \\
& (1+t)=1,078 \\
& t=0,078=7,80 / 0
\end{aligned}
$$

Formule (6). - Si notre propriétaire avait laissé ses $200 \mathrm{fr}$. à la caisse d'épargne à $30 / 0$, il lui aurait fallu 50 ans pour en obtenir $900 \mathrm{fr}$.

$$
\begin{gathered}
\text { Log. } 1,03=0,01283 \\
\frac{0,65321}{0,01283}=50 \text { ans environ }
\end{gathered}
$$

Les tarifs I et II ne peuvent servir qu'aux problèmes (1) et (3), mais cependant par des tâtonnements, ils conduisent les personnes qui ignorent le calcul des logarithmes à une solution approximative des questions (5) et (6), car le taux des placements forestiers dépasse rarement 5 pour cent.

Exemple: Une plantation de pins qui vient d'être effectuée a une valeur de $400 \mathrm{fr}$. l'hectare; on prévoit qu'à 30 ans la coupe vaudra $900 \mathrm{fr}$. l'hectare (5 stères par an $=150$ et à $6 \mathrm{fr}$. l'un) et que l'immeuble vaudra ainsi 1,300 fr. l'hectare, à quel taux l'argent a-t-il été placé pendant ces 30 ans ?

$$
\begin{gathered}
400 \times(1+x)^{30}=1300 \\
(1+x)^{30}=\frac{1300}{400}=3,250
\end{gathered}
$$

En consultant le tarif I sur la ligne de 30 ans, on voit que le taux s'approche de $40 / 0$ qui a le facteur 3,243 . 
Combien d'années faudrait-il pour que la mise de fonds, $400 \mathrm{fr}$., devienne $1,300 \mathrm{fr}$. à $30 / 0$ ?

En parcourant la colonne du $30 / 0$, on voit que le facteur 3,250 tombe entre 39 et 40 ans, tout près de 40 ans.

$\mathrm{N}^{\circ}$ 30. - La relation entre le capital générateur $\mathrm{F}$ et le revenu $R$

$$
\mathrm{R}=\mathrm{F}\left[(1+t)^{n}-1\right]
$$

fournit de la même manière trois autres problèmes qui sont :

$1^{\circ}$ Le capital générateur du revenu

$$
\mathrm{F}=\frac{\mathrm{R}}{(1+t)^{n}-1}
$$

$2^{\circ}$ Le taux du placement

$$
\log \cdot(1+t)=\frac{\log \cdot(R+F)-\log \cdot F}{n}
$$

$3^{\circ}$ Le nombre d'années de la périodicité pour un taux connu

$$
n=\frac{\log \cdot(\mathrm{R}+\mathrm{F})-\log \cdot \mathrm{F}}{\log \cdot(1+t)}
$$

Nous avons considéré la formule (4) comme donnant le prix du fonds générateur (sol et matériel d'exploitation); c'est fort exact quand on donne à $t$ la valeur du taux auquel $\mathrm{F}$ a fonctionné pour fournir $\mathrm{R}$, ainsi

$$
\frac{700}{(1,078)^{20}-1}=200 \mathrm{fr} \text {. }
$$

C'est bien 200 francs qui est la valeur du sol et de sa plantation, ou du sol ensouché. Mais si on veut calculer le prix du sol avec cette formule, en se servant du taux habituel des placements en biens fonds, trois pour cent, par exemple, on aurait

$$
700 \times 1,240=868 \mathrm{fr} . \quad(\text { tarif } I I I)
$$

qui est de la pure fantaisie, car les prix des terrains sont donnés par. les ventes faites dans la localité, c'est dire 
qu'ils tiennent à la loi de l'offre et de la demande qui est différente pour chaque village et pour chaque nature de terrain; un taux de placement moyen mesure cette loi, mais avec des différences énormes d'un cas à l'autre. Il mesure les prix, comme les moyennes dans les statistiques expriment la valeur d'une chose dans une localité ( $\left.\mathrm{n}^{\circ} 29\right)$.

$N^{\circ} 31$. - On a vu que nous avons deux formules pour exprimer le capital initial

$$
\begin{gathered}
\mathrm{P}=\frac{\mathrm{M}}{(1+t)^{n}} \\
\mathrm{~F}=\frac{\mathrm{R}}{(1+t)^{n}-1}
\end{gathered}
$$

A vrai dire, elles ne signifient pas la même chose et ne correspondent pas au même ordre d'idées. La première donne la valeur actuelle du capital futur et transfère une propriété limitée au temps pendant lequel il est formé. La seconde fournit le capital générateur d'un revenu déterminé qui se reproduit tous les $n$ ans : elle donne le prix d'une propriété perpétuelle.

Les deux expressions ne seront identiques que si on y introduit le taux même qui a servi à former le capital futuri; elles donneront des résultats dissemblables si on les calcule avec un taux identique mais choisi arbitrairement.

Reprenons l'exemple du no 24 : Un taillis coùtant $400 \mathrm{fr}$. l'hectare exploité, valant $1,125 \mathrm{fr}$. à 35 ans et ayant produit par conséquent $725 \mathrm{fr}$. en 35 ans.

Le capital initial sera :

$$
\begin{gathered}
\mathrm{P}=\frac{1125}{(1,03)^{3 \mathrm{~s}}}=1125 \times 0,355(\text { tarif II })=400 \mathrm{fr} . \\
\mathrm{F}=\frac{725}{(1,03)^{35}-1}=725 \times 0,551(\text { tarif III })=400 \mathrm{fr} .
\end{gathered}
$$

Cette identité de valeur tient à ce que le taux de formation du capital est précisément $30 / 0$, car

$400 \times(1,03)^{35}=400 \times 2,813(\operatorname{tarif} \mathrm{I})=1125 \mathrm{fr}$. $400 \times(1,03)^{35}-1=400 \times 1,813($ tarif $I-1)=725 \mathrm{fr}$. 
Mais prenons un taux quelconque, par exemple $20 / 0$, l'expression

$$
\mathrm{P}=\frac{1125}{(1,02)^{35}}=562 \mathrm{fr} \text {. }
$$

ne représentera pas plus le capital initial que la formule

$$
\mathrm{F}=\frac{725}{(1,02)^{35}-1}=726 \mathrm{fr} \text {. }
$$

ne fournira la valeur du sol.

La première donnera bien la valeur actuelle du capital futur $1,125 \mathrm{fr}$. escompté à $20 / 0$; la seconde la valeur d'achat d'un revenu périodique de $725 \mathrm{fr}$. pour un acheteur qui voudrait faire emploi de ses capitaux à $20 / 0$. On concoit que les deux choses ne sont pas identiques; elles ne le seraient que si 1,125 et $725 \mathrm{fr}$. avaient été produits par le même taux et si l'escompte et l'achat étaient faits à ce taux.

Reprenons également l'exemple du $\mathrm{n}^{0} 29$ : Un taillis d'aunes coûtant 200 francs l'hectare à sa plantation, valant 900 fr. à 20 ans et ayant ainsi produit $700 \mathrm{fr}$.

Le taux de formation des 900 francs est 7,8 0/0, la valeur actuelle sera bien à ce taux

$$
\mathrm{P}=\frac{900}{(1,078)^{20}}=200 \mathrm{fr} .
$$

la valeur d'achat sera également

$$
\mathrm{F}=\frac{700}{(1,078)^{20}-1}=200 \mathrm{fi} \text {. }
$$

mais si quelqu'un veut escompter à $30 / 0$ ce bien qui vaudra $900 \mathrm{fr}$. dans 20 ans, il en donnera

$$
\mathrm{P}=\frac{900}{(1,03)^{20}}=500 \mathrm{fr}
$$

Si quelqu'un veut acheter ce revenu périodique de $700 \mathrm{fr}$. en se contentant de $30 / 0$ pour la rémunération de son argent, il en donnera

$$
\mathrm{P}=\frac{700}{(1,03)^{20}-1}=868 \mathrm{fr}^{2} .
$$

Comment se fait-il que ces deux acheteurs, se contentant tous deux du même taux $(30 / 0)$ pour leur argent, en vue de la même échéance (20 ans), offrent, l'un la somme la plus faible (500 fr.) pour la plus grosse échéance (900 fr.), et l'autre une somme beaucoup plus forte $(868 \mathrm{fr}$.) pour une échéance beaucoup plus faible (700 fr.).

C'est que le premier n'est qu'un escompteur qui n'achète qu'un seul et unique produit, tandis que le second va devenir 
propriétaire d'une valeur qui se reproduira indéfiniment; le premier achète une propriété limitée, le second une propriété perpétuelle. Il n'y a donc rien d'étonnant à ce que celui-ci paye relativement plus cher un produit moindre ; l'avenir fait la différence.

Voilà un résultat qui est dû à l'introduction du loyer perpétuel du capital dans la formule (4), tandis que la formule (2) ne contient que la formation limitée du capital futur.

Cette différence explique avec quelle circonspection il faut faire usage des formules d'intérêt et combien la connaissance exacte de leur théorie est nécessaire à l'économiste. Bien des forestiers ont erré et ont pris, l'une pour l'autre, ces formules si différentes, dans les conceptions qu'ils ont fournies sur l'estimation du sol et du taux des placements en forêts.

Notre paysan champenois, de l'exemple cité no 29 , s'y est mépris quand, calculant à quelle limite il devrait s'arrêter pour avoir un placement à $30 / 0$, dans son achat d'aunaie, il n'a pas voulu dépasser $400 \mathrm{fr}$. l'hectare. Il a employé le calcul de l'escompteur au lieu de celui du proprietaire. Il peut aller jusqu'à $768 \mathrm{fr}$. l'hectare et $y$ ajouter $100 \mathrm{fr}$. pour la plantation; il aura un placement à $30 / 0$ assis en propriété perpétuelle tant qu'il ne dépassera pas 868 fr. l'hectare.

Nous ne voulons pas revenir sur ce que nous avons dit des estimations de forêts, mais on conviendra que cette difficulté dans l'emploi des formules mathématiques doit faire préférer les méthodes simples et dégagées de toute complication.

L'observation que nous venons de faire sur la différence de résultats fournis par les deux formules du capital initial, s'applique également à celles du taux ou de la durée.

La formule (7) donne le taux au placement perpétuel, lorsque $\mathrm{F}$ représente le prix d'achat du terrain, la valeur réelle et vénale du fonds générateur; la formule simi- 
laire (5) donne le taux du ptacement temporaire pendant $n$ ans, lorsque $\mathrm{P}$ ne représente que le capital initial conduisant à M. Ce qui arrivera, par exemple, pour des arbres isolés dont le terrain occupé est variable et lorsqu'on cherche le taux du placement, abstraction faite du terrain. Toutes deux donneront le même résultat quand $\mathrm{P}$ et $\mathrm{F}$ ont la même valeur, ce qui arrive dans les peuplements forestiers inséparables du terrain fixe qu'ils occupent.

La formule (8) donnera pour un taux connu, un revenu et un fonds déterminés, la durée de la périodicité de ce revenu. La formule similaire (6) fournira le nombre d'années pendant lequel un capital initial connu a acquis une valeur déterminée, quand il est placé temporairement à un taux connu. Elles donneront toutes deux le même résultat, si $\mathrm{P}=\mathrm{F}$, c'est-à-dire si le capital initial placé temporairement est le même que le fonds générateur.

No 32. - Résumé des intérêts composés :

Relations entre le capital actuel et le capital futur.

Nontant. $\mathrm{M}=\mathrm{P}(1+t)^{n} \quad(\operatorname{tarif} I)(1)$

Valeur actuelle.

$$
\mathrm{P}=\frac{\mathrm{M}}{(1+t)^{n}} \quad(\text { tarif } I I)
$$

Taux du placement temporaire.

$\log .(1+t)=\frac{\log \cdot \mathrm{M}-\log . \mathrm{P}}{n}$

Durée du placement temporaire.

$$
n=\frac{\log \cdot M-\log \cdot P}{\log \cdot(1+t)}
$$

Relations entre le fonds générateur et le revenu.

\section{Revenu.}

$\mathrm{R}=\mathrm{F}\left[(1+t)^{n}-1\right]($ tarif $I-1)(2)$

Fonds générateur.

$$
\mathrm{F}=\frac{\mathrm{R}}{(1+t)^{n}-1} \quad(\text { tarif } 1 I I)
$$

Taux du placement perpétuel.

$\log \cdot(1+t)=\frac{\log \cdot(\mathrm{R}+\mathrm{F})-\log \cdot \mathrm{F}}{n}(7)$

Durée de la périodicité du revenu.

$$
n=\frac{\log \cdot(\mathrm{R}+\mathrm{F})-\log \cdot \mathrm{F}}{\log \cdot(1+t)}
$$


ARTicle 3.

\section{Loi de formation par les annuités.}

Nos 33. - Premier problème. - Montant ou valeur future de $n$ annuités.

34. - Deuxiène problème. - Inutilité de calculer le loyer produit.

35. - Troisième problème. - Principal ou valeur actuelle génératrice de $u$ annuités.

36. - Quatrième problème. - Son inutilité.

37. - Questions dérivées du problème du montant : économie annuelle, feuille, rente annuelle équivalente, valeur des peuplements en croissance.

38. - Nombre d'années nécessaires pour former un capital par économies annulles.

39. - Taux auquel les économies ont fonctionné.

40. - Questions dérivées dı problème de la dette : amortissement.

41. - Temps nécessaire à l'amortissement d'une dette.

42. - Taux auquel la dette a été consentie.

43. - Résumé des formules.

$\mathrm{N}^{\circ}$ 33. - Premier problème (montant de $n$ annuités). - Dans ce mode de formation des capitaux, une somme annuelle constante $a$ s'ajoute chaque année en produisant des intérêts qui se composent, c'est-à-dire qui s'ajoutent aux intérêts des intérêts et à ceux des annuités.

Le montant de $n$ annuités, c'est-à-dire le capital formé dans l'avenir par $n$ sommes égales grossies de leurs intérêts composés, est

$$
\mathrm{M}=a \frac{(1+t)^{n}-1}{t}
$$

En effet, la $1^{\text {ro }}$ annuité $a$ devient $a(1+t)$, la $2^{\circ} a(1+t)^{2}$, la dernière $a(1+t)^{n}$; la somme de ces annuités ainsi grossies n'est autre que la somme des termes d'une progression géométrique dont le ler terme est $a$ et la raison $(1+l)$. Cette somme s'exprime par la formule eonnue

$$
\mathrm{S}=\frac{\alpha\left(q^{n}-1\right)}{q-1}
$$

dans laquelle il suffit de remplacer $\alpha$ par $a$ et $q$ par $(1+t)$ pour avoir l'équation (9). 
Il n'est pas inutile de remarquer que le capital formé s'obtient en multipliant l'annuité par l'intérêt composé qu'elle produit pendant $\mathrm{n}$ ans et en multipliant le résultat par le denier de capitatisation $\frac{1}{t}$ correspondant au taux du placement.

On pourrait construire un tarif donnant le montant de $n$ annuités de 1 franc, mais un tel tarif devient inutile avec les trois qui précèdent et qui sont suffisants pour résoudre toutes les questions de la théorie des intérêts. On voit, en effet, que ce montant est donné par

(Tarif $I-1) \times($ for terme du tarif III)

Exemple. - Un père de famille fait tous les ans 1,000 fr. d'économies et les remet à un banquier qui lui bonifie un intérêt de $21 / 20 / 0$. Quelle somme aura-t-il au bout de 30 ans?

$$
1000 \times 1,098 \times 40=43920
$$

Ce père de famille n'a pas confiance dans le banquier et préfère acheter, chaque année, deux hectares de terrain qui lui coùteront $400 \mathrm{fr}$. l'un et qu'il sèmera en pins au moyen d'une dépense de $100 \mathrm{fr}$. par hectare, ce qui emploiera son économie annuelle de 1,000 fr. Il espère qu'au bout de 30 ans chaque hectare de pins lui procurera 1,000 fr. Au bout de 30 ans, il aura constitué un immeuble de 60 hectares qui vaudra

Sol : 60 hectares à $400 \mathrm{fr}$............. $24000 \mathrm{fr}$.

60 peuplements de 1 à 60 ans, valant en moyenne $450 \mathrm{fr}$. l'hectare .............. 27000

Valeur de la propriété......... $\overline{51000 \mathrm{fr}}$.

Il aura un revenu de 2,000 fr. par an, dont il retranchera $200 \mathrm{fr}$. pour la plantation, soit 1,800 fr. de revenu net, de sorte que son opération lui aura créé un immeuble formant placement à $31 / 20 / 0$.

$$
\frac{1800}{51000}=0,035
$$

La forêt est donc un aussi bon capitalisateur d'épargnes que le banquier.

Autre exemple. - - Un propriétaire possède 30 hectares de terrains ordinaires estimés $500 \mathrm{fr}$. et qui lui produisent $30 \mathrm{fr}$. 
de fermage annuel par hectare. Il imagine d'en planter un chaque année en pins, ce rui le privera d'un revenu de $30 \mathrm{fr}$. et lui imposera une dépense de $100 \mathrm{fr}$. chaque année.

Placée à la caisse d'épargne, cette économie do $100 \mathrm{fr}$. aurait produit en 30 ans, à $30 / 0$ :

$$
100 \times 1,427 \times 33,33=4756
$$

Le sacrifice de 30 revenus de 30 fr. lui coûte, au même taux :

$$
30 \times 1,427 \times 33,33=1426
$$

Il se sera ainsi imposé un sacrifice de $6,182 \mathrm{fr}$., mais il aura créé une propriété garnie de 30 peuplements de 1 à 30 ans, valant, à $450 \mathrm{fr}$. l'un en moyenne, la somme de $13,500 \mathrm{fr}$.

$\mathrm{Au}$ bout des 30 ans, sa situation sera donc celle-ci :

En revenu, il avait un fermage de 30 hectares à $30 \mathrm{fr}$., valant $900 \mathrm{fr}$. La forêt lui rapporte chaque annẻe 1 hectare de coupe valant 1,000 fr., sur lequel il prélève $100 \mathrm{fr}$. pour le semis. C'est le mème levenu sans les mauvaises chances d'insolvabilitẻ des fermier's agricoles.

En capital : il possédait 30 hectares agricoles valant $500 \mathrm{fl}$., soit 15,000 fr. La forèt sera toujours estimée au moins :

Sol : 30 hect. $\times 500 \ldots \ldots \ldots \ldots \ldots \ldots \ldots, 15000$ fr.

Superficie : 30 hect. $\times 450 \ldots \ldots \ldots \ldots \ldots 13500$

Son patrimoine est devenu....... $\overline{28500 \mathrm{fr}}$.

La création d'une forêt n'est donc pas toujours une mauvaise opération, même quand elle est faite sur des terres agricoles ayant en apparence un bon rapport; car, dans cet exemple, on s'est imposé un sacrifice de 6,181 francs pour créer un immeuble qui a une plusvalue de 13,500 francs.

$\mathrm{N}^{\circ} 34$. - Le deuxième problème que nous avons examiné dans la formation des capitaux par l'intérêt composé et qui consiste à déterminer l'intérêt produit par le capital pourrait de même se poser.

Dans le premier exemple du numéro précédent on a déboursé, en réalitè, trente annuités de 1,000 francs et l'intérêt produit serait :

$$
43920-30000=13920 \mathrm{fr} .
$$


Mais si ces 30,000 francs forment bien le déboursé total, c'est un déboursé successif échelonné d'années en années.

Ce n'est pas le capital initial qui a produit 43,920 francs et il faudrait une r'éduction à l'actualité pour donner à ces trentes annuités leur valeur réelle. La complication qui en résulterait pour exprimer l'intérêt produit serait tout à fait stérile. Le problème est utile, au contraire, dans la formation des capitaux par la loi des intérêts composés ; il a d'ailleurs conduit aux quatre tarifs (réduits à trois) qui sont l'auxiliaire indispensable de tous les calculs relatits à la théorie de l'intérêt.

Nous ne nous occuperons donc pas de cette recherche qui tend à dégager du montant futur l'intérêt produit par la valeur réelle du capital initial et qui serait $\mathrm{M}-\mathrm{P}$ ou l'expression résultant de la soustraction des seconds termes des équations $(9)-(10)$. (Voir no 35 .)

$\mathrm{N}^{\circ} 35$. - Troisième problème (principal de $n$ annuités). - Quelle est la valeur actuelle de $n$ annuités? Par ce problème, on a pour but de chercher à quel prix on achèterait au comptant un certain nombre d'annuités quand on veut faire emploi de ses fonds à un taux $t$.

On voit que pour ce calcul, chaque annuité doit être ramenée à l'actualité pour son temps d'échéance. Le montant $\mathrm{M}$ exprimant toutes ces annuités condensées à l'expiration des $n$ ans; la valeur actuelle de cet ensemble sera donc le montant ramené à l'actualité pour $n$ ans.

$$
\mathrm{P}=\frac{\mathrm{M}}{(1+t)^{n}}
$$

Si on remplace $M$ par sa valeur en fonction de $a$ on aura

$$
\mathrm{P}=a\left(\frac{(1+t)^{n}-1}{t} \times \frac{1}{(1+t)^{n}}\right)
$$


ou

$$
\mathrm{P}=a \frac{(1+t)^{n}-1}{t(1+t)^{n}}
$$

C'est donc l'intérêt d'une annuité pendant $\mathrm{n}$ ans multiplié par le denier, le tout ramenéà l'actualité pour $\mathrm{n}$ ans; c'est, en d'autres termes, le montant de n annuités ramené à l'actualité pour n ans (').

Quelques auteurs ont calculé un tarif à différents taux, pour obtenir le principal ou la valeur actuelle de $n$ annuités $\left({ }^{2}\right)$, un pareil tarif est bien inutile puisqu'on obtient le facteur cherché par les tarifs I, II et III.

$($ Tarif $I-1) \times\left(f^{\mathrm{er}}\right.$ termie du tarif $\left.I I I\right) \times($ tarif $I I)$

Exemples. - $1^{\circ}$ Un pont à péage, ayant encore 30 ans de privilège, produit 1,000 fr. par an. Le propriétaire ne consent à racheter qu'à $21 / 20 / 0$ : quelle somme faut-il lui payer?

ou le montant

$$
1000 \times 1,098 \times 40 \times 0,477
$$

A $50 / 0$, il faudrait lui payer

$$
43920 \times 0,477=20950 \mathrm{fr} .
$$$$
1000 \times 3,322 \times 20 \times 0,231
$$

ou le montant

$$
66440 \times 0,231=15347 \mathrm{fr} .
$$

$2^{\circ}$ Une action de jouissance d'une compagnie de chemin de fer doit encore rapporter 15 francs par an pendant 40 ans; combien doit-elle ètre cotée au taux de $40 / 0$ ?

$$
15 \times 4,801 \times 25 \times 0,208=374 \text { fr. } 40
$$

$3^{\circ}$ Un droit d'affectation au profit d'une usine est de 200 stères à 5 fr. ou de 1,000 fr. par an; elle doit encore durer 20 ans; on veut en affranchir la forêt par un cantonnement

(1) On arriverait à la démonstration de la formule par la roie plus parlante aux yeux qui consiste a remarquer que l'expression $\frac{a}{1+t}+\frac{a}{(1+t)^{2}}$ $+\ldots \frac{a}{(1+t)^{n}}$ est la somme des termes d'une progression géométrique dont le premier est a et la raison $\frac{1}{1+t}$.

$i^{2}$ ) Tarif $V$ des ouvrages publiés par MM. Cotla, de Salomon el Nanquette. 
réglé au taux légal de $50 / 0$ (Cod. f., art. 58), quelle sera la valeur du canton de forêt à céder à l'usinier ?

$$
1,000 \times 1,653 \times 20 \times 0,37 \tau=12,463 \mathrm{fr} .
$$

Il faudra céder à l'affectataire un canton de forêt valant actuellement en fonds et superficie 12,463 francs, c'est-à-dire d'une valeur telle que s'il la vendait, il en retirerait cette somme.

On voit que le calcul du principal de $n$ annuités donne à la fois l'escompte et le fonds générateur et qu'il n'y a pas à faire la distinction que nous avons remarquée dans l'intérêt composé, puisque l'annuité est toujours à temps limité.

$N^{\circ} 36$. - Quatrième problème. - Quant à la recherche du capital générateur de l'intérêt produit par des annuités s'accumulant pendant $n$ années, elle serait par les raisons indiquées $\left(n^{0} 34\right)$ tout à fait inutile.

Il ne faut pas confondre ce problème de pure spéculalion mathématique avec celui qui a pour but de chercher le capital générateur au taux $t$ des annuités pendant un temps limité. Il est résolu par la formule du principal de $n$ annuités ( $\left.n^{\circ} 35\right)$ - ni avec le problème qui consiste à trouver le capital générateur d'une annuité a constante et perpétuelle. Ce problème a été résolu au tarif III quand la période se réduit à un an (ou $n=1$ ).

$$
\mathrm{F}=a \frac{1}{t}
$$

Le facteur $\frac{1}{t}$ n'est autre chose que le denier de capitalisation correspondant au taux $t$, c'est le premier terme du tarif III. Ainsi le capital générateur de 100 francs à $40 / 0$ serait

$$
100 \times 25=2500 \text { francs. }
$$

Son emploi suppose naturellement que le premier revenu sera touché au bout de la première année et non au 
début. Si, par exception, on le touchait au début, la valeur du capital serait

$$
a\left(\frac{1}{t}+1\right) \text { ou } a \frac{1+t}{t}
$$

Dans l'exemple ci-dessus : 2600 francs.

$\mathrm{N}^{\circ}$ 37. - La théorie des annuités se réduit, en réalité, à deux problèmes: $1^{\circ}$ le montant ou valeur future; $2^{\circ}$ le principal ou valeur actuelle.

Mais à la différence de la formation par intérêts composés, la relation n'existe plus entre la valeur future et la valeur actuelle, mais entre chacune de ces valeurs et l'annuité qui est l'élément constitutif de la loi de formation.

Une autre différence, c'est que le problème de la valeur actuelle fournit à la fois la valeur de l'escompte et celle du fonds générateur de $n$ annuités ; que si, par conséquent, on voulait établir des tarifs pour cette loi de formation ils seraient réduits à deux, analogues aux tarifs I et II de l'intérêt composé. Le tarif III n'aurait pas de similaire.

Nous avons quelques détails à donner sur le problème du montant qu'on a souvent appelé dans les écoles : problème de la Dot, parce qu'il s'agit d'un capital à former par opposition avec le problème du principal, dit problème de la Dette.

$1^{\circ}$ De la formule du montant

$$
\mathrm{M}=a \frac{(1+t)^{n}-1}{t}
$$

on déduit que l'économie annuelle nécessaire pour former une dot ou un capital $M$ en $n$ ans, en la versant tous les ans à un banquier qui fournit un intérêt composé au taux $t$, est :

$$
a=\mathrm{M} \frac{t}{(1+t)^{n}-1}
$$

Formule qui se résout par

$$
\text { tarif } I I I \times t
$$


On prend par exemple 3 pour cent du chiffre fourni par le tarif III, si l'emploi doit être fait à ce taux.

Application. - $1^{\circ}$ Un père de famille veut économiser chaque année une certaine somme, et la placer à $50 / 0$, pour établir un enfant qui vient de naitre; il se demande quelle économie lui est nécessaire pour former au bout de 20 ans la somme de 20,000 franes.

$$
20000 \times 0,605 \times 0,05=605 \mathrm{fr} .
$$

à $30 / 0$, l'économie nécessaire aurait été de

$$
20000 \times 1,240 \times 0,03=744 \mathrm{fr} .
$$

Rien n'est plus simple que ce calcul puisqu'il suffit de prendre 5 ou 3 pour cent du capital générateur d'une rente périodique de 20,000 francs tous les 20 ans.

Le facteur ainsi formé :

$$
\begin{aligned}
50 / 0 \text { de } 0,605 & =0,0302 \\
\text { ou } 30 / 0 \text { de } 1,204 & =0,0361
\end{aligned}
$$

n'est autre que l'annuité formant un capital de 1 franc en un certain temps: en rapportant ce facteur à cent francs on a 3,02 et 3,61 pour cent. On l'appelle le pour cent du capital à former selon que le taux du placement est de 5 ou de 3 pour cent.

$2^{\circ}$ La comparaison de ce qui se passe dans les forêts où la coupe se reproduit avec périodicité a fait penser qu'on pourrait par ce moyen représenter la pousse annuelle d'un hectare de bois et calculer ce qu'on a appelé la feuizle, c'est-à-dire, cette quantité constante qui s'ajoute à ellemême en se grossissant de ses intérêts pour constituer la coupe.

Voici, par exemple, un taillis dont la coupe vaut 725 fr. à 35 ans ; quelle est la valeur de la feuille?

On a d'abord pris un taux arbitraire d'accroissement, $50 / 0\left(^{1}\right)$ ce qui a donné

$$
725 \times 0,2214 \times 0,05=8,02
$$

( ${ }^{1}$ Tarif ou compte fait de la valeur progressive d'un hectare de taillis, par M. Chabanne, inspecteur des forêts, br. in-40, Paris, 1831. 
Les valeurs successives ont reçu alors les prix suivants :

A 1 an................. 802

A 2 ans................. 1644

A 3 ans................. 2528

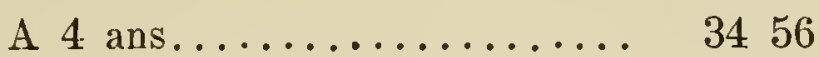

A 5 ans................. 4430

A 6 ans................... 5453

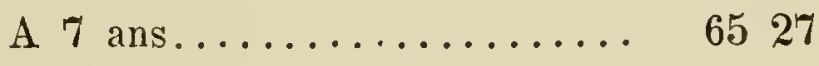

A 35 ans............... 725 »

Les feuilles annuelles avaient les valeurs ci-après :

A 1 an.................. 802

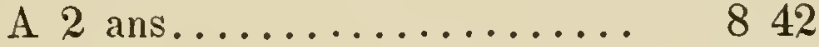

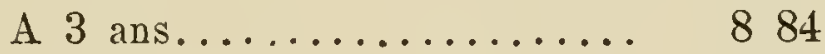

A 4 ans........................... 928

A 5 ans.................. 974

A 6 ans.................. 1023

A 7 ans................. 1074

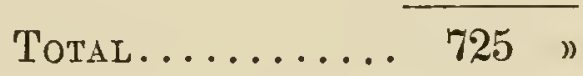

Cette échelle de valeurs est en harmonie avec ce qui se passe dans la nature, mais si la végétation ajoute chaque année au bois des valeurs de plus en plus grandes, sa loi d'accroissement n'est certainement pas celle des annuités. Quant à choisir le taux arbitraire de 5 pour cent pour mesurer cette marche déjà fictive, c'est de la pure fantaisie.

On a cru se rapprocher davantage de la réalité en calculant le taux d'accroissement du bois d'après la valeur du terrain prise comme capital initial, en d'autres termes, d'après le taux réel du placement des fonds engagés dans l'exploitation forestière.

D'après les ventes de terres faites dans la localité, le terrain vaut par exemple $400 \mathrm{fr}$. l'hectare, la valeur produite en 35 ans est de 1,125 (sol $400+$ taillis 725 ) c'est un accroissement à $30 / 0$ à intérêts composés; ce cálcul préliminaire effectué $\left(\mathrm{n}^{\circ} 30\right.$, formule 7$)$ on a déterminé la première feuille à $12 \mathrm{fr}$.

$725 \times 0,551=400 \mathrm{fr} . \times 0,03=12 \mathrm{fr}$. 
Cette manière d'opérer est assurément plus légitime que celle qui se sert d'un taux arbitrairement choisi, mais elle repose toujours sur une inexactitude, c'est que la loi de la végétation n'est pas celle des annuités. Toutefois, il faut reconnaître que cette méthode, appliquée dans ces conditions, c'est-à-dire avec le taux même de formation du capital, est moins mauvaise pour représenter la feuille; on ne peut lui reprocher que de se fonder sur une hypothèse inexacte : celle qui consiste à dire que la valeur des peuplements croît suivant la loi des annuités ou suivant la loi des intérêts composés.

Il est digne de remarquer, en effet, que ces deux lois sont identiques du moment où on a pris pour mesure le taux qui a servi à passer, à intérêts composés, de la valeur du fonds (sol et matériel) à la valeur de la coupe.

Reportons-nous à l'exemple précédent: un terrain vendu $400 \mathrm{fr}$. produisant une coupe de $725 \mathrm{fr}$. à 35 ans, ce qui correspond à $30 / 0$ de placement et à $30 / 0$ de formation pendant la période de 35 ans $\left(n^{\circ} 30\right)$. Les valeurs progressives de la proprièté boisée du taillis et de la feuille seront :

\begin{tabular}{|c|c|c|c|}
\hline & Propriété. & Taillis. & Feuille. \\
\hline A 1 an.. & 412 & $12 》$ & $12 》$ \\
\hline A 2 ans.. & 42436 & 2436 & 1236 \\
\hline A 3 ans. & 43708 & 3708 & 1272 \\
\hline A 4 ans, etc. & 45020 & 5020 & 1312 \\
\hline
\end{tabular}

C'est identiquement ce que donnerait le calcul par l'annuité, car dans ce calcul effectué pour un taux ainsi déterminé, l'annuité sera de $12 \mathrm{fr}$. et le fonds sera

$$
12 \times 33,33=400 \mathrm{fr} \text {. }
$$

Cette observation est faite pour montrer :

a) Que les méthodes d'estimation fondées sur la loi des intérêts composés et sur celle des annuités sont identiques;

b) Que si on emploie le taux du placement de la forêt, les valeurs initiale et finale sont seules exactes;

c) Que même dans ce cas, les valeurs intermédiaires 
sont inexactes, car la végétation suit une toute autre loi de formation;

d) Que les valeurs intermédiaires ainsi calculées représentent exactement le prix que les choses ont pour leur propriétaire;

e) Que toutes les valeurs sont inexactes quand on se sert du taux moyen des immeubles ruraux ou même forestiers dans la localité;

f) Que, enfin, ce calcul par annuités entraîne des complications qu'on doit chercher à éviter pour se garantir contre les erreurs, car les meilleurs esprits n'en ont pas toujours été affranchis $\left({ }^{1}\right)$.

$3^{\circ} \mathrm{La}$ formule qui nous occupe et qui donne l'économie, l'annuité constitutive d'un capital futur a été considérée comme la transformation en revenu annuel d'un revenu périodique. En d'autres termes, cette annuité à servir pendant $n$ années serait le revenu annuel équivalent à un revenu intermittent et perpétuel qui se reproduit tous les $n$ ans. Or, ce n'est que la rente du fonds générateur, déduction taite des intérêts du capital engagé ( $\left.n^{\circ} 150\right)$.

On en a déduit $\left({ }^{2}\right)$ un moyen de comparer le revenu agricole qui est annuel au revenu forestier qui est périodique (voir Estimations forestières, p. 92, n 58).

Dans notre taillis acheté $400 \mathrm{fr}$. après la coupe et produisant $725 \mathrm{fr}$. en 35 ans, l'annuité constitutive de $725 \mathrm{fr}$. à $30 / 0$ a été appelée rente du sol ou rente foncière; elle est :

$$
725 \times \frac{0,03}{(1,03)^{35}-1}=12 \mathrm{fr}
$$

Si l'hectare de même valeur vénale, aux environs, rapporte $20 \mathrm{fr}$. cultivé ou $10 \mathrm{fr}$. en pâture, on dit que la propriété forestière est inférieure à la propriété agricole et supérieure à l'immeuble pastoral.

(1) Une circulaire de l'Administration des forèts du 20 mai 1831, no 275 , a transmis aux agents les comptes faits à $\ddot{0} 0 / 0$ par $\mathbf{~}$. Chabanne, pous l'estimation en fouds et superficic des forêts.

$\left.{ }^{2}\right)$ Gustave IJeyer, Anleitung zur Waldwerthechnng, Leipzig, 1876. 
Ce raisonnement n'est exact qu'à la double condition de calculer la feuille sur le taux de placement de la forêt et de considérer uniquement certaines forêts à exploitation périodique. Il est inexact quand on l'étend aux forêts aménagées et il demande, en outre, un soin tout particulier dans son application ( $\mathrm{n}^{\circ} 150$ ).

Alors il faut bien reconnaître qu'il est parfaitement inutile de recourir à cette complication : le taux du placement de l'exploitation agricole et celui de l'exploitation forestière suffisent à toutes les comparaisons.

$\mathrm{Si}$, par exemple, partant de ce que les immeubles se vendent en moyenne à $40 / 0$, on calculait l'annuité équivalente à 725 fr. sur cette base

$$
725 \times \frac{0,04}{(1,04)^{3 \ddot{s}}-1}
$$

$725 \times 0,3394 \times 0,04=9$ fr. $85($ tarif III $\times t)$ et si, en comparant aux champs qui se louent $20 \mathrm{fr}$. et aux pâturages qui produisent $10 \mathrm{fr}$., on concluait à une inférioritè de la forêt, à la fois sur les champs et sur les pâturages, on commettrait une erreur : celle-ci provient de ce qu'en agissant ainsi on a assigné au fonds une valeur de $246 \mathrm{fr}$.

$$
\frac{725}{(1,04)^{35}-1}
$$

$725 \times 0,3394=246 \mathrm{fr}$. (tarif III)

tout à fait arbitraire et beaucoup trop faible puisqu'il a été acheté $400 \mathrm{fr}$.

Il faut donc toujours partir du prix du terrain tel qu'il a été acheté, ou de sa valeur vénale résultant des transactions locales, et en déduire le taux de placement.

Partir d'un taux arbitraire, même le mieux raisonné, c'est donner à la terre une valeur fictive qui échappe à toute argumentation. Mais alors, si, partant de la valeur vénale du terrain et lui comparant la valeur de la coupe, on en déduit le taux de formation du capital, qui est aussi le taux du placement ( $\mathrm{n}^{\circ} 30$ ), il est bien inutile de faire intervenir ici une formule d'annuités, grosse de complications et donnant prise à l'erreur: la forêt donnée rapporte 
$30 / 0$; le terrain se vendrait $400 \mathrm{fr}$.; son produit annuel est donc de 12 fr., c'est-à-dire inférieur ou supérieur aux loyers des cultures voisines qui sont de 20 ou de $10 \mathrm{fr}$. C'est aussi simple que cela, et la formule des intérêts composés suffit à toutes les exigences.

$4^{\circ} \mathrm{La}$ formule de la feuille

$$
a=\mathrm{M} \frac{t}{(1+t)^{n}-1}
$$

a été généralisée, c'est-à-dire qu'on en a déduit une expression $x$ donnant la valeur d'un peuplement en croissance, ayant l'âge $m$ en fonction du produit $\mathrm{M} q \mathrm{u}^{\prime}$ on obtiendrait à l'âge d'exploitation $n$ au taux du placement de la forêt.

$$
x=\mathrm{M} \frac{(1+t)^{m}-1}{(1+t)^{n}-1}
$$

Cette formule s'explique d'elle-même, car le peuplement âgé de $m$ ans n'est pas autre chose que l'intérêt dans $m$ ans $\left[(1+t)^{m}-1\right]$ du fonds générateur $\frac{\mathrm{M}}{(1+t)^{m}-1}$. C'est la généralisation de la formule (11).

On l'a appelée formule de la superficie, expression du peuplement en croissance. Cela repose toujours sur l'hypothèse que la valeur des peuplements s'accroît suivant la loi des intérêts composés au taux du placement de la forêt, ce qui n'est pas vrai d'une manière absolue (erga omnes) et n'est exact que pour l'acheteur de la forêt (erga dominum). Cela suppose aussi que le peuplement est exploité en entier à chaque coupe, comme dans les taillis ordinaires: lorsque, au contraire, la superficie n'est exploitée qu'en partie, comme dans les taillis sous futaie, la formule ne donne que la valeur de la partie de la superficie qui doit entrer dans le revenu (récolte pendante) et laisse en dehors la partie réservée pour former le matériel d'exploitation (souches et balivage). 
On a été ainsi conduit à estimer la valeur des forêts, en fonds et superficie, par les deux formules suivantes:

$$
\begin{aligned}
& \mathrm{S}=\mathrm{R} \frac{(1+t)^{m}-1}{(1+t)^{n}-1} \\
& \mathrm{~F}=\mathrm{R} \frac{1}{(1+t)^{n}-1}
\end{aligned}
$$

A l'égard desquelles il convient de remarquer :

a) Que $\mathrm{S}$ ne représente pas toute la superficie, mais seulement la partie de la superficie qui constitue la récolte en croissance;

b) Que $\mathrm{F}$ ne représente pas le sol nu, mais ce sol garni de son matériel reproducteur (souches, semis, arbres de réserve);

c) Que la valeur ainsi fournie est relative à celui qui veut faire emploi de son argent à un taux déterminé.

$5^{\circ}$ On voit avec quelle circonspection ces formules d'intérêts doivent être employées. Aussi, on a souvent commis, pour évaluer les peuplements en croissance, une autre erreur que nous devons signaler: elle consiste à ramener à l'actualité, par la formule du tarif II, la valeur du peuplement à la coupe.

Si le taillis vaut $725 \mathrm{fr}$. à 35 ans, le peuplement devra être escompté à $30 / 0$; il sera :

à 34 ans, $725^{\mathrm{f}}$ escompté pour 1 an, soit $703^{\mathrm{f}}$ »

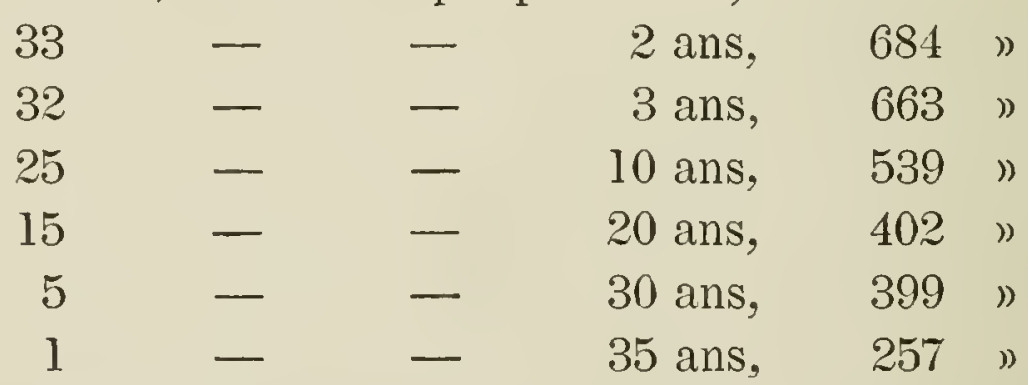

Ce raisonnement est tout à fait erroné ; vainement dirait-on que le taillis de 1 an est un véritable billet de 725 francs à échéance de 35 ans, qu’il vaut réellement 257 francs pour celui qui veut employer son argent à 
$30 / 0\left(n^{\circ} 24\right)$ et que si ce taux de $30 / 0$ est celui du placement de la forèt, on a, par ce moyen, la valeur des peuplements en croissance.

Tout cela repose sur une confusion: il ne faut pas confondre la manière dont le capital complet s'accroît avec la facon dont le peuplement seul s'augmente. Le capital passe de 400 francs à 1,125 en 35 ans; en réunissant ces deux sommes pour la relation des intérèts composés, on a d'abord le taux de formation qui est aussi le taux de placement, et on a, ensuite, les valeurs capitales successives de l'inmeuble à ses différents âges, de 412 à 1,125 francs.

Il en résulte qu'en retranchant le capital (ce qui donne les valeurs $12 ; 24.36 ; 37.08 ; 50.20 \ldots$ à 725 ) on obtient une expression assez exacte de la valeur des peuplements. Ceux-ci sont, en effet, le produit d'un sol valant $400 \mathrm{fr}$. Mais escompter 725 francs pour $1,2,3 \ldots 35$ ans, c'est supposer que l'accroissement des peuplements se fait comme un capital de 257 trancs placé à intérêts composés à $30 / 0$, pour devenir 725 francs, tandis que ces peuplements proviennent d'un capital de 400 francs devenu 1,125 trancs au bout de 35 ans. C'est armettre que l'accroissement du produit se fait suivant la même loi et au même taux que l'augmentation du capital et que la végétation progresse de la même manière que l'immeuble, ce qui est une conception de pure fantaisie $\left(n^{\circ} 30\right)$. Tout ce que l'on peut dire, c'est que les valeurs actuelles de 257 .. à 725 francs, représentent la récolte pendante au prix que celle-ci a pour un acheteur non propriétaire. Celui-ci a ses fonds engagés dans nne exploitation qui lui rapporte $30 / 0$ : le priver de la première coupe, par une expropriation, par exemple, c'est lui enlever un billet à échéance de 35 ans et qui vaut 257 francs pour l'acheteur de cette récolte.

On voit qu'il y a loin de cette valeur toute relative et 
personnelle à la valeur vénale et courante des peuplements en croissance.

$\mathrm{N}^{\circ} 38$. - La formule du montant de $n$ annuités fournit encore la solution de la question suivante:

Pendant combien d'années faut-il payer une annuité $a$ pour former un capital futur M à un taux $t$.

$$
n=\frac{\log \cdot(\mathrm{M} t+a)-\log \cdot a}{\log \cdot(1+t)}
$$

Exemple. - Une commune a le projet de construire un jour une maison d'école de $50,000 \mathrm{fr}$. et ne veut pas faire d'emprunt; la coupe de la forêt a été jusqu'alors délivrée aux habitants; le conseil municipal décide une taxe affouagère [de $4,000 \mathrm{fr}$. et ordonne que cette taxe sera chaque année versée au Trésor public qui sert un intérêt de $30 / 0$. Le conseil demande en combien d'années la somme de 50,000 fr. sera acquise et pendant combien d'années, par conséquent, la taxe affouagère devra exister.

$$
\begin{aligned}
& \text { N } t 50000 \times 0,03=1500 \\
& \text { a........... }=4000 \\
& \log . \overline{5500}=3,7403627 \\
& \log .4000=\frac{3,6020600}{0,1383027} \\
& \log .(1,03)=\frac{0,1383027}{0,0128372}=10^{\text {ans }} 3 / 4
\end{aligned}
$$

$\mathrm{N}^{\circ}$ 39. - Enfin, cette même formule du montant permet d'arriver au taux suivant lequel l'argent a fonctionné quand on connaît l'annuité servie, la somme produite et le temps pendant lequel on a payé l'annuité.

De la formule fondamentale

$$
\mathrm{M}=a \frac{(1+t)^{n}-1}{t}
$$

on ne peut pas tirer $(1+t)$ ni directement, ni par logarithmes parce que $t$ est dans les deux membres, mais on déduit

$$
\frac{a}{M}=\frac{t}{(1+t)^{n}-1}
$$


Cette formule donne $t$ par tâtonnement de la facon la plus facile avec le tarif III, si le taux ne dépasse pas 5 pour cent; autrement il faudrait faire des essais directs.

Exemple. - Un père de famille a versé 1,000 fr. par an, pendant 20 ans, à une compagnie d'assurances, dans le but de constituer une dot à sa fille : à la majorité de celle-ci, la compagnie lui remet $30,000 \mathrm{fr}$. ; le père veut savoir à quel taux la compagnie l'a traité.

Il effectue d'abord le calcul très simple

$$
\frac{1000}{30000}=0,0333
$$

premant ensuite sur la ligne 20 les facteurs du tarif Ill

$$
1,2405 \quad 1,0103 \cdot 0,8395 \quad 0,708
$$

il les multiplie par les taux correspondants

ce qui donne

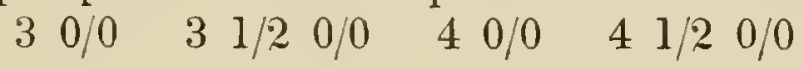

$$
0,0361 \quad 0,0353 \quad 0,0335 \quad 0,0318
$$

Il roit ainsi que le taux qu'on lui a servi tombe entre 4 et $41 / 2$, beaucoup plus près de 4 que de $41 / 2$. Il n'a pas lieu d'ètre très satisfait de la gestion de la compagnie qui ne l'a pas fait grandement profiter des clances de mortalité qui ont dủ se produire chez les co-assurés de sa fille.

$\mathrm{N}^{\circ}$ 40. - Le problème fondamental de la dette ou du principal de $n$ annuités fournit à son tour trois problèmes dérivés qui répondent aux questions d'amortissement comme ceux qui dérivent du problème du montant ont donné les solutions relatives aux économies nécessaires pour constituer un capital.

De la formule

$$
\mathrm{P}=a \frac{(1+t)^{n}-1}{t(1+t)^{n}}
$$

on déduit, en effet,

$$
a=\mathrm{P} \frac{t(1+t)^{n}}{(1+t)^{n-1}}
$$

formule qui répond à la question suivante: Quelle somme annuelle faut-il payer au créancier pour se libérer en $n$ ans d'une dette $\mathrm{P}$ contractée au taux $t$ ? 
Elle se résout par les tarifs I et III.

$$
\text { (tarif } I) \times(\text { tarif } I I I) \times(t)
$$

Exemple. - Une commune, propriétaire de bois, a promis une subvention de $150,000 \mathrm{fr}$. à une compagnie de chemin de fer. Pour la payer, elle demande une coupe extraordinaire de pareille somme dont l'effet sera désastreux pour la forêt. L'agent forestier lui conseille d'emprunter $150,000 \mathrm{fr}$. au Crédit foncier à $41 / 20 / 0$, amortissable en 30 ans. On lui demande quelle annuité il faudra payer pendant 30 ans pour être libéré.

$$
\begin{gathered}
3,7453 \times 0,3643 \times 0,045=0,0614 \\
0,0614 \times 150000=9210 \mathrm{fr} .
\end{gathered}
$$

Au moyen de cette sommo de 9,210 fr. que la forêt peut fournir pendant 30 ans, la commune sera libérée de sa dette et la forêt sera conservèe, car les arbres sur pied continueront à s'accroitre en attendant chaque coupe extraordinaire.

On voit que le facteur calculé par les tarifs I et III est, en réalité, le pour cent d'anortissement de 1 franc en 30 ans. On dit alors que l'amortissement se fait à 6,14 0/0 pour un emprunt à 4 1/2 en 30 ans $\left({ }^{1}\right)$.

L'annuité pour amortir 100 francs en 30 ans est toute différente de l'annuité pour former 100 francs; la première est plus considérable que la seconde et le public dit avec raison qu'il est plus facile d'économiser que de payer sa dette. La raison vient de ce que celle-ci n'est pas une simple somme à payer, mais cette somme grossie de ses intérêts, c'est le total, capital et intérêts, qu'il faut solder. Dans l'économie, au contraire, il ne faut que constituer la somme.

Ainsi, pour former 150,000 francs en 30 ans, à 4 1/2 0/0, il faudra économiser 2,460 francs ou....... $1,640 / 0$

Pour se libérer de 150,000 francs en 30 ans, au même taux, il faudra payer tous les ans 9,210 francs ou ................. 6,140/0

( ${ }^{1}$ Le Crédit foncier demande souvent une commission de 0,30 , ce qui porterait le taux d'amortissement à 6,44 ; ce taux est toutefois un peu moindre, parce que le Crédic foncier compose les intérèts par semestre et non par année. 
Il suffit de considérer la formule de l'économie

$$
a=\mathrm{M} \frac{t}{(1+t)^{n}-1}
$$

et celle de l'amortissement

$$
a=\mathrm{P} \frac{t}{(1+t)^{n}-1} \times(1+t)^{n}
$$

On voit que la première est en fonction du montant et l'autre du principal, qui ne sont jamais identiques, car la valeur actuelle d'une dette est toujours beaucoup plus faible que la dette elle-même. Si, comme nous l'avons fait dans l'exemple ci-dessus, on suppose la dette à amortir et le capital à former égaux entre eux, l'annuité d'amortissement sera naturellement plus forte que l'économie de création. Il faudra multiplier celle-ci par $(1+t)^{n}$, en effet:

$$
1,64(1,045)^{30} \text { ou } 1,64 \times 3,745=6,14
$$

Le créancier qui recevra $9,210 \mathrm{fr}$. pendant 30 ans et qui les placera à intérêts composés à $41 / 20 / 0$, réaliserait en 30 ans la somme énorme de $561,750 \mathrm{fr}$.

$$
\mathrm{M}=2,745 \times 22,22 \times 9210=561750 \mathrm{fr} .
$$

Effectivement la valeur de cette grosse somme, ramenée à l'actualité, serait $150,000 \mathrm{fr}$.

$$
\mathrm{P}=2,745 \times 22,22 \times 0,266 \times 9210=150000 \mathrm{fr} .
$$

ll y a entre cette valeur actuelle de 30 annuités de 9,210 francs et le montant futur de 30 annuités de la même somme, la même différence qu'entre l'économie pour créer ou pour amortir des sommes égales.

Le principal doit être multiplié par $(1+t)^{n}$ pour reproduire le montant:

$$
\mathrm{M}=\mathrm{P}(1+t)^{n}
$$

Pour s'en convaincre il suffit de transporter clans la formule du montant (9) la valeur de $a$ en fonction du principal (13).

Ainsi, quelle que soit la manière dont un capital a été formé, par annuités ou par intérêts composés, la relation entre le capital futur et sa valeur actuelle est la même. 
Cela démontre une fois de plus ( $\left.n^{\circ} 37\right)$ que ceux qui ont cru trouver dans la loi des annuités une méthode d'estimation des forêts supérieure à celle des intérêts composés, n’ont fait que compliquer la question et les calculs pour arriver au même résultat.

Cela explique aussi que lorsque nous avons apprécié ces méthodes d'estimation ( $\left.{ }^{1}\right)$, nous n’avons pas parlé du procédé des annuités et n'avons examiné que la méthode des intérêts composés.

Une dernière observation : on est toujours conduit dans la théorie des annuités à distinguer l'économie pour créer la valeur future, de l'annuité pour se libérer. Lorsque l'économie et l'annuité sont exprimées, l'une en fonction de la valeur future $\mathrm{M}$, comme dans (9), l'autre en fonction de la valeur actuelle $P$, comme dans (13), les deux formules donnent bien le même résultat et la lettre $a$ indique bien des sommes identiques.

$$
a=\mathrm{M} \frac{t}{(1+t)^{n}-1}=\mathrm{P} \frac{t(1+t)^{n}}{(1+t)^{n}-1}
$$

Mais si on considère une somme quelconque $\mathrm{C}$, soit à créer ou à amortir, l'économie et l'amortissement sont différents et il faut les distinguer par une notation spéciale.

$$
\begin{aligned}
& \varepsilon=\mathrm{C} \frac{t}{(1+t)^{n}-1} \text { (Economie, feuille des forêts) } \\
& \alpha=\mathrm{C} \frac{t(1+t)^{n}}{(1+t)^{n}-1} \text { (Amortissement) }
\end{aligned}
$$

C'est pour n'avoir pas fait cette distinction qu'on a souvent erré dans une théorie qui est, en réalité, assez simple.

$\mathrm{N}^{\circ}$ 41. - La formule de la dette (10) fournit le moyen

(1) Estimations concernant la propriété forestière, chap. I. 
de connaître en combien d'années une dette contractée P au taux $t$, sera amortie par le paiement d'une annuité déterminée $a$.

On en déduit, en effet:

$$
n=\frac{\log \cdot a-\log \cdot(a-\mathrm{P} t)}{\log \cdot(1+t)}
$$

Exemple: La commune qui désire construire une maison d'école de 50,000 fir. au moyen d'une taxe affouagère de $4,000 \mathrm{fr}$. (n1 38$)$, a reconnu qu'en versant cette taxe à la caisse du Trésor public à $30 / 0$ elle aura ses $50,000 \mathrm{fr}$. en 10 ans $3 / 4$. Elle se demande si elle ne veut pas emprunter cette somme pour jouir tont de suite de l'école. Elle trouve un prêteur qui lui offre $50,000 \mathrm{fr}$. à $50 / 0$ et qui demande la garantie de la taxe affouagère pendant tout le temps qui sera nécessaire pour amortir la dette; quel est ce temps ?

$$
\begin{aligned}
\log .4000 & =3,60206 \\
50000 \times 0,05=2500 & =\frac{3,17609}{0,42597} \\
\log .1500 & =20 \text { ans } 1 / 10 \\
\log .1,05 & =\frac{0,02119}{1,00}
\end{aligned}
$$

La commune trouve que cette aliénation de l'affouage pendant 20 ans est bien longue. Si elle rencontrait un prèteur à $40 / 0$, l'annuité de $4,000 \mathrm{fr}$. mettrait 17 ans $1 / 2$ à la convrir. Enfin, un prêt à $30 / 0$ serait remboursé par le service de cette anunité pendant 15 ans environ.

Le problème est naturellement impossible quand $a<\mathrm{P} t$, car les nombres négatifs n’ont pas de logarithmes. Il est évident qu'on n'amortirait jamais en payant une annuité plus faible que l'intérêt de la dette et que loin de se libérer, on ne ferait que grossir son obligation.

$N^{\circ}$ 42. - Enfin le même problème fondamental de la dette permet de connaître à quel taux a été consenti un prêt déterminé $\mathrm{P}$ quand on connaît l'annuité $a$ à servir et le nombre des années $n$ pendant lesquelles on doit la payer. 
Cette question de taux ne pent se résoudre en arithmétique que par tâtonnements.

$$
\frac{a}{\mathrm{P}}=\frac{t(1+t)^{n}}{(1+t)^{n}-1}
$$

On essaiera différentes valeurs pour $t$ et celle qui fournira un résultat égal à $\frac{a}{\mathrm{P}}$, correspondra au taux cherché. Les tarifs I et III permettent de laire rapidement ce tâtonnement.

Exemple: La commune citée au numéro précédent songe à la caisse des écoles qui lui demandera 2,000 fr. (4 0/0 de $50,000 \mathrm{fr}$.) pendant 30 ans et qui lui donne l'assurance que moyennant ce service de 2,000 fr. pendant 30 ans elle sera libérée. La commune se demande à quel taux est fait cet emprunt :

$$
\frac{2000}{50000}=0,040
$$

Cherchant ensuite sur la ligne 30 du tarif I pour les taux :

\begin{tabular}{lllll} 
& \multicolumn{1}{c}{1} & $11 / 2$ & \multicolumn{1}{c}{2} & $21 / 2$ \\
les facteurs.......... & 1,347 & 1,563 & 1,811 & 2,097 \\
les multipliant par..... & 0,01 & 0,015 & 0,02 & 0,025 \\
ce qui donne.......... & 0,0134 & 0,0234 & 0,0362 & 0,0524 \\
ensuite par tarif III..... & 2,874 & 1,775 & 1,232 & 0,911 \\
ce qui produit......... & 0,0385 & 0,0415 & 0,0446 & 0,0447
\end{tabular}

On voit que le facteur $\frac{a}{\mathrm{P}}$ tombe entre 0,0385 et 0,0415 correspondant au taux de 1 et $11 / 20 / 0$, ce qui établit un taux de $11 / 40 / 0$.

C'est ce que décide, au surplus, l'article 13 de la loi du 3 juillet 1880 disposant que les prêts seront faits aux communes à $11 / 4$ pour cent par an, en vue de la construction des écoles publiques. Devant un sacrifice aussi considérable fait par l'État et avec des avantages pareils, les communes auraient bien tort de compromettre leurs forêts par des coupes extraordinaires. 
No 43. - Résumé des annuités :

Relation entre l'annuité $a$ et le capital futur $\mathrm{M}$.

Montant (dot).

$\mathrm{M}=a \frac{(1+t)^{n}-1}{t}(\operatorname{tarif} I-1) \times \frac{1}{t}(9)$

Economie (feuille).

$a=\mathbb{M} \frac{t}{(1+t)^{n}-1}($ tarif $I I I) \times t$

Durée de formation.

$$
n=\frac{\log \cdot(\mathrm{M} t+a)-\log \cdot a}{\log \cdot(1+t)}
$$

Taux du placement.

$$
\frac{a}{\mathrm{M}}=\frac{t}{(1+t)^{n}-1}
$$

Relation entre l'annuité $a$ et le capital actuel $\mathrm{P}$.

Principal (dette).

$\mathrm{P}=a \frac{(1+t)^{n}-1}{t(1+t)^{n}}(T . I-1)(T . I I) \frac{1}{t}(10)$

Amortissement.

$$
a=\mathrm{P} \frac{t(1+t)^{n}}{(1+t)^{n}-1}(T . I)(T . I I I) \times t
$$

Durée de l'amortissement.

$$
n=\frac{\log \cdot a-\log \cdot(a-\mathrm{P} t)}{\log \cdot(1+t)}
$$

Taux de la dette.

$$
\frac{a}{\mathrm{P}}=\frac{t(1+t)^{n}}{(1+t)^{n}-1}
$$

Fonds générateur.

$$
\mathrm{F}=\alpha \frac{1}{t}
$$

Formule des estimations relatives.

$$
\begin{aligned}
& \text { Récolte pendante }=\mathrm{R} \frac{(1+t)^{m}-1}{(1+t)^{n}-1} \\
& \text { Fonds générateur }=\mathrm{R} \frac{1}{(1+t)^{n}-1} \\
& n=\text { âge d'exploitation. } \\
& m=\text { âge du moment. } \\
& \mathrm{R}=\text { revenu. }
\end{aligned}
$$


Article 4.

\section{Loi de formation par les annuités périodiques.}

Nos 4. - Problème du montant d'un certain nombre $n$ d'annuités pério. diques.

43̈. - Problème du principal de $n$ annuités.

46. - Capital générateur d'annuités périodiques.

47. - Résumé des formules.

$\mathrm{N}^{\circ}$ 44. - Il y a des produits qui ne durent qu'un temps limité pendant lequel ils se reproduisent d'une facon sensiblement égale, mais périodique. La valeur produite dans l'avenir est le résultat d'une annuité $a$ à intermittence $p$ pendant np années au taux $t$.

Cette valeur se calcule par la formule

$$
\mathrm{M}=a \frac{(1+t)^{n p}-1}{(1+t)^{p}-1}
$$

qui s'explique par analogie de celle du montant des annuités annuelles, car en faisant, dans celle ci-dessus, $p=1$, on retombe sur (9).

Ces produits se rencontrent rlans les forêts, mais ce n'est pas uniquement en vue des coupes principales ou des éclaircies naturellement périodiques que ce calcul a été introduit dans les estimations des produits forestiers. La loi des intérêts composés suffit à résoudre les questions qui y sont relatives. Ceux qui ont introduit dans les calculs forestiers les annuités périodiques, ont eu en vue certains produits comme la glandée, la fainée, la résine, l’émondage des arbres.

Exemple. - Dans une futaie de hêtre et chêne exploitée à 150 ans, la fainée qui se reproduit assez généralement tous les 10 ans commence dans les hauts perchis à 70 ans; il y a ainsi 
8 récoltes qui se vendent 6 fr. par hectare: quelle est la valeur produite par ces 8 récoltes à $20 / 0$ ?

$$
\begin{aligned}
& \mathrm{M}=6 \frac{(1,02)^{80}-1}{(1,02)^{10}-1} \quad(\text { tarif } I-1) \times(\text { tarif III }) \\
& \mathrm{M}=6 \times 3,875 \times 4,566=106 \mathrm{fr} .20
\end{aligned}
$$

La valeur de l'annuité intermittente serait, connaissant la valeur future produite $\mathrm{M}$

$$
\begin{gathered}
a=\mathrm{M} \frac{(1+t)^{p}-1}{(1+t)^{n p}-1} \\
106,20 \times 0,220 \times 0,258=6 \mathrm{fr} .
\end{gathered}
$$

Mais ce calcul n'a qu'un intérêt de pure curiosité arithmétique, ainsi que les autres déductions qu'on pourrait tirer de la formule (17).

No 45. - On peut chercher de même quelle est la valeur actuelle, le principal de $n$ annuités à intermittence $p$, durant np années : elle sera par analogie

$$
\mathrm{P}=a \frac{(1+t)^{n p}-1}{(1+t)^{p}-1} \times \frac{1}{(1+t)^{n p}}
$$

Dans l'exemple précédent, les 80 annuités à 10 ans d'intervalle, valant dans l'avenir $106 \mathrm{fr}$., auraient une valeur actuelle de

$$
106 \times 0,205=21 \text { fr. } 70 \quad \text { (tarif } 11 I)
$$

Les autres formules qu'on serait tenté d'en déduire par analogie, n'ont également point d'intérêt.

No 46. - Quant aux annuités périodiques d'une durée illimitée, telles que les coupes qui se produisent tous les $2,3,4, \ldots$ années, elles ne sont que des loyers de capitaux placés à intérêts composés pendant la durée de la période. C'est ainsi que leur capilal générateur est

$$
\mathrm{F}=a \frac{1}{(1+t)^{p}-1}
$$

formule qui est identique avec celle du tarif III. 
Ce n'était donc pas la peine d'arriver à la conception si compliquée des annuités intermittentes, pour retomber en définitive sur la loi des intérêts composés; celle-ci suffit à toutes les exigences des estimations forestières, à la seule condition qu'on sache en faire un emploi raisonné.

Voici un exemple du calcul des annuités périodiques:

Dans la futaie de hêtre et chène à 15() ans, valant à cet âge $7,400 \mathrm{fr}$., sur un sol de $400 \mathrm{fr}$. l'hectare, l'accroissement s'est fait à $20 / 0$; l'annuité représentative de la feuille est ainsi de 8 fr. $\left(n^{\circ} 44\right)$

$$
a=740 \frac{0,02}{(1,02)^{150}-1}=8 \mathrm{fr} .
$$

mais cette somme ne représente pas la véritable et seule rente annuelle du sol, car il faut lui ajouter ses produits à intermittence limitée.

Le produit des 80 annuités de fainée à 6 fr. l'une, ramené à l'actualité, vaut $21 \mathrm{fr} .70$; il se l'eproduira ainsi avec sa valeur' initiale tous les 150 ans et équivaut donc à un capital générateur de

et par an

$$
21,70 \times 0,054(\text { tarif III) }=1 \mathrm{fr} .17
$$

$$
1,17 \times 0,02=0,023
$$

ce capital devra s'ajouter à la valeur du sol et la porter à $401 \mathrm{fr}$. 17, et la rente Ảu sol deviendra elle-même 8 fr. 023 .

Véritablement, les auteurs qui, par amour de la scrupuleuse exactitude, ont introduit dans les estimations de forêts ces annuités périodiques, n’ont pas craint les complications.

Ce n'est pas tout: cette valeur principale des 80 annuités de fainée est ramenée à l'actualité de 70 ans, âge où elle commence, tandis que celle du produit principal a été ramenée au début de l'âge du bois, à 1 an; c'est comme si cette valeur actuelle $21 \mathrm{fr}$. 70 était grevée d'usufruit pendant 70 ans et ne commençait à fonctionner au profit du propriétaire de la forêt que dans 70 ans. Il y a donc un correctif et une réduction à l'actualité à introduire pour 70 ans : c'est dire que cette fainée a une valeur tout à fait négligeable. 
Toutefois, les suspensions de jouissance qui se présentent notamment dans les forêts grevées d'usufruit doivent être exprimées par le calcul au moyen de formules déduites de la théorie des intérêts composés et que nous allons expliquer.

No 47. - Résumé des annuités périodiques.

Montant ou capital futur.

$\mathrm{N}=a \frac{(1+t)^{n p}-1}{(1+t)^{p}-1}$

Principal ou capital actuel.

Fonds générateur.

$$
\mathrm{F}=a \frac{1}{(1+t)^{p}-1}
$$

Article כ.

\section{Usufruit.}

Nos 48. - Calcul de la valeur de l'usufruit : 10 d'une rente annuelle; 20 d'une rente périodique.

49. - Valeur de la nue propriété dans ces deux cas.

50 . - Usufruits concédés sous condition suspensive.

31. - Formule générale des estimations de forèts.

52. - Procédés équivalents mais plus compliqués assignant des valeurs distinctes alt fonds et à la superficie; méthode de l'escompte.

ว3. - Résumé des lormules.

54. - Observation.

$\mathrm{N}^{\circ}$ 48. - Deux valeurs distinctes sont à considérer dans les biens grevés d'usufruit : la nue propriété et l'usufruit. Comme ces attributs sont tous deux cessibles, il faut en rechercher le prix d'estimation pour celui qui veut faire emploi de son argent à un taux déterminé.

Nous estimerons d'abord l'usufinit.

Deux cas sont à considérer : le bien est à revenu annuel 
ou à revenu périodique, mais tous deux sont limités dans leur durée puisque la jouissance usufructuaire finit, soit avec la vie du bénéficiaire (ce qui est alors une question de mortalité), soit avec le terme assigné par la convention ou par la loi.

$1^{\circ}$ On veut vendre à un acquéreur qui cherche emploi de son argentau taux $t$ un usufruit à produit annuel $a$ devant durer $n$ ans. On demande la valeur de cet usufruit.

Le prix est évidemment la valeur de $u$ annuités:

$$
\begin{gathered}
\mathrm{U}=a \frac{(1+t)^{n}-1}{t(1+t)^{n}} \\
\text { (tarif } I-1) \times(\text { tarif } I I) \times \frac{1}{t}
\end{gathered}
$$

$2^{\circ}$ On veut vendre au taux $t$ l'usufruit d'une rente $a$ intermittente, c'est-à-dire se reproduisant tous les $p$ ans et devant durer $n p$ années; quel est le prix de cet usufruit?

C'est ici encore la valeur actuelle de $n$ annuités périodiques

$$
\begin{gathered}
\mathrm{U}=a \frac{(1+t)^{n p}-1}{(1+t)^{p}-1} \times \frac{1}{(1+t)^{n p}} \\
(\text { tarif } I-1) \times(\text { tarif } I) \times(\text { tarif } I I I)
\end{gathered}
$$

$\mathrm{N}^{\circ}$ 49. - La valeur de la nue propriété, dans ces deux cas, suppose une rente annuelle ou périodique perpétuelle qui serait ramenée à l'actualité pour tout le temps $s$ que dure la suspension des produits.

$1^{\circ}$ Un bien à revenu annuel $a$ qui ne doit commencer à courir que dans $s$ ans est en vente. Quelle est la valeur pour celui qui veut faire cmploi de son argent au taux $t$ ?

Cette valeur sera $\left({ }^{1}\right)$

$$
\begin{gathered}
\mathrm{P}=a\left(\frac{1}{t} \times \frac{1}{(1+t)^{s}}\right) \\
(\text { tarif } I I) \times \frac{1}{t}
\end{gathered}
$$

(1) Cette formule est l'objet d'un tarif spécial, dit tarif IV, dans les ou- 
$2^{\circ}$ Un bien à revenu périodique $a$ se reproduisant tous les $p$ ans d'une façon perpétuelle est grevé d'usufruit pendant un temps $s$; la rente $a$ ne conmencera à courir que dans $s$ ans: quelle est la valeur de ce bien au taux $t$ ?

$$
\begin{aligned}
\mathrm{P}= & a\left(\frac{1}{(1+t)^{p}-1} \times \frac{1}{(1+t)^{s}}\right) \\
& (\text { tarif } I I) \times(\text { tarif } I I)
\end{aligned}
$$

On vérifiera les formules de l'usufruit et de la nue propriété en les ajoutant ensemble et en faisant $s=n$, c'est-àdire en faisant la durée de l'usufruit égale à la durée de la suspension dans les produits; on trouvera que la propriété entière non grevée est :

$$
\begin{gathered}
\frac{1}{t} \text { dans le premier cas } \\
\frac{1}{(1+t)^{p}-1} \text { dans le second cas : }
\end{gathered}
$$

Ce sont les facteurs du tarif III.

$N^{0}$ 50. - Enfin, il y a des usufruits concédẻs sous une condition suspensive, c'est-à-dire qu'ils ne doivent commencer à courir qu'après un temps $s$. La valeur de ces usufruits $U^{\prime}$ ainsi reculés sera pour l'usufruit d'une rente annuelle $a$ devant durer $n$ ans et ne commencant qu'après $s$ ans.

$$
\mathrm{U}^{\prime}=a \frac{(1+t)^{n}-1}{t(1+t)^{n}} \times \frac{1}{(1+t)^{s}}
$$

vrages de MM. Cotta, de Salomon et Nanquette. Ce tarif IV a été introduit dans les livres d'estimation des forèts à l'époque où les défrichements étaient favorisés par la haute valeur de la terre agricole et oì il fallait tenir compte dans l'estimation de la forêt de lia possibilité d'une transformation en culture. Il suftit, dans ce cas, derenu beaucoup plus rare, d'évaluer le temps $s$ pendant lequel se ferait la réalisation du matériel boisé et d'estimer le capital dû au revenu agricole annuel sous déduction d'un escompte pendant $s$ ans. Le tarif' II et le premier facteur du tarif III y sultisent sans qu'il soit besoin d'un tarif spécial. 
et pour l'usufruit d'une rente à période $p$ derant durer nj) ans et ne commençant qu'après $s$ années.

$$
\mathrm{U}^{\prime}=a \frac{(1+t)^{n p}-1}{\left[(1+t)^{p}-1\right](1+t)^{n p}} \times \frac{1}{(1+t)^{s}}
$$

C"est aussi le cas de deux usufruits successifs grevant un bien l'un après l'autre. La valeur de ces usufruits et celle de la nue propriété qui leur correspond se calculeront toujours facilement arec les trois tarifs qui sont le fondement de la théorie des intérêts. Il suffira d'un peu de réflexion pour décomposer les valeurs.

No 51. - Ces problèmes de l'usufruit ont été introduits dans l'estimation des forèts quand le revenu périodique diun bois ne se produit pas immédiatement, mais subit un certain temps de suspension.

Reprenons l'exemple cité $11^{\circ} 21$ d'un taillis produisant 725 fr. à 35 ans, sur un sol qui raut, la coupe faite et le terrain ensouché, $400 \mathrm{fr}$. l'hectare, ce qui indique une formation de la raleur à raison de 30,0 à intérèts composés $\left(n^{\circ} 30\right)$.

Cette somme de $400 \mathrm{fr}$. est en effet fournie par :

$$
\begin{gathered}
\mathrm{F}=\mathrm{R} \frac{\mathrm{l}}{(1,03)^{35}-1} \\
725 \times 0,551=400 \mathrm{fr} .
\end{gathered}
$$

Toilà la valeur de l'immeuble immédiatement après la coupe, pour celui qui rent faire emploi de son argent à 30,0 : il n’y a pas de superficie à éraluer.

Supposons maintenant que cet hectare de terrain soit garni de sa coupe. c'est-ì-dire soit âgé actuellement de 35 ans.

L'immeuble raudra

$$
\begin{gathered}
400+725=1125 \mathrm{fr} \\
\text { ou } 725\left(\frac{1}{(1+t)^{35}-1}+1\right)
\end{gathered}
$$

ou comme nous l'arons ru au no 28

$$
725 \frac{(1+t)^{35}}{(1+1)^{35}-1}=1125 \mathrm{fr}
$$


Supposons enfin, que le bois soit actuellement âgé de 4 ans; on aura la valeur de l'immeuble en remarquant que le capital générateur provient d'une rente périodique de 725 fr. qui ne doit commencer à fonctionner que dans 31 ans, mais qui, au bout de cette suspension de fonctionnement, livrera tout d'abord sa rente; ce sera commo si l'hectar'e de 35 ans, bon à couper, était reporté, dans sa jouissance, à 31 ans, ou était grevé d'usufruit pendant 31 ans.

On aura la valeur de l'immeuble en ramenant à l'actualité pour 31 ans l'expression précédente

$$
725 \frac{(1+\imath)^{35}}{(1+\imath)^{35}-1} \times \frac{1}{(1+l)^{31}}=450 \mathrm{fl}^{\circ} .
$$

ou, plus généralement, et en désignant par $n$ l'âge de la coupe ( $35 \mathrm{ans}$ ), par $s$ la durée de la suspension (31 ans), par $\mathrm{R}$ le revenu tous les $n$ ans et par $\mathrm{V}$ la valeur capitale cherchée

$$
\mathrm{V}=\mathrm{R} \frac{(1+t)^{n-s}}{(1+t)^{n}-1}
$$

Or, $n-s$ n'étant autre chose que l'âge du bois $m$ au moment actuel, on a la formule suivante, très employée dans les estimations de forêts :

$$
\mathrm{V}=\mathrm{R} \frac{(1+t)^{m}}{(1+t)^{n}-1}
$$

Elle donne le résultat cherché par le seul usage du tarif I et du tarif III

$$
725 \times 0,551 \times 1,125=450 \mathrm{fr} .
$$

et se traduit par la règle suivante :

Pour avoir la valeur relalive d'un canton de forêt qui n'a pas encore l'âge de la coupe, il faut multiptier le revenu à l'exploitation par le facteur du tarif I comespondant ì l’âge actuel et le résultat obtenu par le facteur du tarif III correspondant à l’age de la coupe.

Naturellement, pour avoir une expression complete de la valeur d'un bois, il faulra supputer, en outre, la valeur $V^{\prime}$ qui est due aux coupes intermédiaires. 
Supposons que ce taillis soit soumis, à l'âge de 25 ans, à une coupe d'éclaircie destinée à dégager les cimes de certains jeunes chènes et à les préparer à fournir de bons baliveaux; admettons que cette coupe inter'médiaire fournisse un produit de $150 \mathrm{fr}$. et appelons

$n$ l'âge d'exploitation principale (35 ans),

$e$ l'âge d'exploitation intermédiaire ( 25 ans),

$m$ l'âge actuel ( 4 ans).

Naturellement cette coupe de $150 \mathrm{fr}$. ne se produira que tous les 35 ans, et si on se place à 25 ans, on touchera immédiatement à cette époque la coupe d'éclaircissement.

$\mathrm{La}$ valeur capitale serait alor's

$$
\mathrm{V}^{\prime}=\mathrm{R}^{\prime} \frac{(1+t)^{n}}{(1+t)^{n}-1}
$$

$\mathrm{Si}$, au lieu de nous placer à l'âge de 25 ans, nous nous reportons au début de l'exploitation, il faudra ramener cette valeur à l'actualité, l'escompter pour $e$ années ( 25 ans). Mais ce n'est pas dans 25 ans qu'on réalisera cette coupe, c'est en réalité dans $25-21$ ans, puisque le bois a maintenant 4 ans, de sorte qu'il faut escompter la valeur précédente, non pour $e$ ans, mais pour $e-m$ ans.

$$
\mathrm{V}^{\prime}=\mathrm{R}^{\prime} \frac{(1+t)^{n}}{(1+t)^{n-1}} \times \frac{1}{(1+t)^{e-m}}
$$

Cette formule qui peut se mettre sous la forme plus simple

$$
\mathrm{V}^{\prime}=\mathrm{R}^{\prime} \frac{(1+t)^{n-e+m}}{(1+t)^{n}-1}
$$

donne la valeur du capital générateur d'une éclaircie et fournit la formule générale des estimations ( $\left.{ }^{1}\right)$; elle se résout par le tarif I et le tarif III. Il faut seulement faire attention de calculer exactement l'exposant du facteur du tarif II : du terme principal d'exploitation, on retranche l'âge de l'éclaircie et on ajoute uu résultat l'âge actuel du bois.

(1) Nous arons montré qu'elle contient tous les cas qui peurent se présenter : Estimations concernant la propriété forestière, 1886, I vol., gr. in-\$o, p. 22. 
Dans l'exemple précédent, on voit que

$$
35-25+4=14
$$

$$
150 \times 0,551 \times 1,512=125 \mathrm{fr} .
$$

De sorte que le bois âgé de 4 ans qui nous sert d'exemple vaut à $30 / 0$ :

$$
\begin{aligned}
& \text { Du chef de la coupe à } 35 \text { ans .... } 450 \mathrm{fr} \text {. } \\
& \text { Du chef de l'éclaircie à } 25 \text { ans.... } \quad 125 \\
& \text { Soit........... }
\end{aligned}
$$

Il ne faut jamais oublier que ce prix est celui que pourrait donner un acheteur voulant faire emploi de ses capitaux à un taux déterminé; c'est une valeur relative et non une valeur vénale ou absolue. D'autres pourront en donner davantage s'ils se contentent de faire rapporter $20 / 0$ à leur argent; des marchands de bois pourront ainsi $\mathrm{y}$ trouver des ressources qui leur permettront d'élever le prix.

$\mathrm{N}^{\circ}$ 52. - La formule qui donne par un seul calcul la valeur d'une forêt en fonction de sa coupe principale

$$
\mathrm{V}=\mathrm{R} \frac{(1+t)^{m}}{(1+t)^{n}-1}
$$

n'est pas autre chose que la condensation du procédé d'estimation séparée pour le fonds et la superficie que nous avons indiqué au $\mathrm{n}^{\circ} 37$

$$
\begin{aligned}
& \mathrm{S}=\mathrm{R} \frac{(1+t)^{m}-1}{(1+t)^{n}-1} \\
& \mathrm{~F}=\mathrm{R} \frac{1}{(1+t)^{n}-1}
\end{aligned}
$$

L'aspect seul de ces expressions à dénominateur commun le montre suffisamment. Le calcul par les tables le prouve également.

Forêt de taillis rapportant 725 fr. à 35 ans, à $30 / 0$, et actuellement âgée de 4 ans.

Valeur de la forèt : $725 \times 0,551 \times 1,125=450 \mathrm{fr}$. $I d e m\left\{\begin{array}{l}\text { Superficie: } 725 \times 0,551 \times 0,125=50 \\ \text { Fonds: } 725 \times 0,551\end{array}\right\} 450 \mathrm{fr}$. 
A vrai dire, cette dernière méthode n'est qu'une manière de disposer les calculs et un procédé d'exécution auquel on a été conduit par le désir que l'on avait d'exprimer séparément les deux éléments de l'immeuble : le sol et la superficie.

On n'est arrivé de cette facon qu'à une analogie, car il faut remarquer, comme nous l'avons déjà fait au $n^{\circ} 37$, que la première expression ne donne pas toute la superficie qui constitue le revenu en croissance; la seconde ne donne pas le sol nu, mais le sol garni de son matériel d'exploitation, souches et arbres de réserve. On a cru éviter cette complication en employant un autre mot et en reconnaissant que la seconde expression fournit non la valeur du sol, mais celle du fonds génératerr. Il y a toutefois un inconvénient notable et même une inexactitude de langage quand on emploie ce procédé de calcul divisé dans les forêts dont le matériel d'exploitation est formé par des arbres ou des parties de peuplements (taillis sous futaie); c'est que ce matériel croît avec l'âge d'une quantité qui fait partie de la récolte; la seconde expression fournissant une valeur toujours constante (celle du sol et du matériel initial de la coupe qui vient d'être exploitée), il en résulte que la division des calculs fournit la récolte pendante et le capital initial : cette division n'exprime donc ni la superficie ni le fonds nu et on arrive à une complication dangereuse pour des estimateurs inexpérimentés.

Certains auteurs ont cru remédier à cet inconvénient par un autre procédé du même calcul qu'ils ont appelé procédé de l'escompte et dans lequel

$$
\begin{gathered}
\text { Superficie }=\mathrm{R} \frac{1}{(1+t)^{n-m}} \\
\text { Fonds }=\mathrm{R} \frac{1}{(1+t)^{n}-1} \times \frac{1}{(1+t)^{n-m}}
\end{gathered}
$$


Dans l'exemple précédent, on a raisonné ainsi:

Pour la coupe principale à 35 ans :

Superficie : Coupe de 725 fr. escomptée pour 31 ans: $725 \times 0,400$ (tarif $I I) \ldots \ldots \ldots \ldots \ldots \ldots \ldots .290 \mathrm{fr}$.

Fonds: Capital d'une rente périodique de $725 \mathrm{fr}$. se reproduisant tous les 35 ans, mais ne commencant à fonctionner que dans 31 ans et devant être ainsi escomptée pour 31 ans : $725>0,551 \times 0,400(l a-$ rif $I I I) \times($ tarif $I I) \ldots \ldots \ldots \ldots \ldots \ldots \ldots \ldots, 160$

Total..................... $450 \mathrm{fr}$.

Et pour l'éclair'cissement à 25 ans :

Superficie: Coupe de $150 \mathrm{fr}$. escomptée pour 21 ans : $150 \times 0,537$ (tarif II) ............

Fonds: Capital générateur de $150 \mathrm{fr}$. tous les 35 ans, escompté pour 21 ans: $150 \times 0,551 \times$ $0,537($ tarif II $)$
Valeur de l'immeuble..............

Par ce procédé, le fonds générateur varie à chaque âge, ce qui paraît en har'monie avec la croissance des réserves; mais alors on a rencontré une autre difficulté, c'est que dans les forêts dont on exploite tout le peuplement (taillis, pineraie, etc.) le fonds qui n'est autre que le sol, prend des valeurs très diverses et que des terrains de même qualité sont estimés à des prix très différents, selon l'âge des coupes, ce que ne peuvent comprendre les personnes qui examinent les estimations faites suivant ce procédé. Il faut, en effet, un gros effort de réflexion et de conception mathématique pour se convaincre que sur la coupe de 4 ans, la valeur du sol est réduite à 160 francs parce qu'elle reste pendant 31 ans sans fonctionner à nouveau.

Les partisans fanatiques de la division des calculs en fonds et superficie ont alors proposé d'employer le premier procédé pour les forêts dont la coupe se fait à blanc estoc, et celui de l'escompte pour les forêts à arbres de réserve. Ce n'est qu'un mirage et une fausse conception: la vérité est que le chiffre total ( $150 \mathrm{fr}$. et $575 \mathrm{fr}$. dans l'exemple ci-dessus) est seul exact et que les éléments dont on l'a 
formé en leur donnant des noms de pure analogie avec le fonds et la superficie, ne sont que des facteur's du calcul du résultat final qui seul doit ètre présenté.

La méthode par la formule unique, que nous avons appelée formule générale d'estimation des forêts, est donc bien plus avantageuse, en ce sens qu'elle oblige à moins de calculs et qu'elle évite surtout les confusions, les erreurs et les mal entendus dont les estimations forestières ont trop souvent donné l'exemple. L'étude des limites et des conditions dans lesquelles cette méthode doit être employée forme l'objet de notre livre consacré aux Estimations concernant la propriété forestière.

No 53. - Résumé de l'usufruit.

Valeur de l'usufruit.

en $n$ annuités.

$$
\mathrm{U}=a \frac{(1+t)^{n}-1}{t(1+t)^{n}}
$$

en $n$ annuités à période $p$.

$$
\mathrm{U}=a \frac{(1+t)^{n p}-1}{(1+t)^{p}-1} \times \frac{1}{(1+t)^{n p}}
$$

Valeur de la nue propriété.

$$
\text { en annuités. }
$$

$$
\mathrm{P}=a \frac{1}{t(1+t)^{s}}
$$

en annuités périodiques.

$$
\mathrm{P}=a \frac{1}{(1+t)^{p}-1} \times \frac{1}{(1+t)^{s}}
$$

Formule générale des estimations relatives en fonds et superficie.

$$
\begin{aligned}
& \text { Pour la coupe principale : } \mathrm{V}=\mathrm{R} \frac{(1+t)^{m}}{(1+t)^{n}-1} \\
& \text { Pour les coupes intermédiaires: } \mathrm{V}^{\prime}=\mathrm{R}^{\prime} \frac{(1+t)^{n}-e+m}{(1+t)^{n}-1} \\
& n=\text { âge d'exploitation principale. } \\
& e=\text { âge de l'exploitation intermédiaire. } \\
& m=\text { âge du moment. }
\end{aligned}
$$

No 54. - Nous nous excusons d'avoir donné un trop long développement à la théorie de l'intérêt. Celle-ci aurait gagné à être exposée plus brièvement et sans incursion dans le domaine purement forestier. Mais il a été commis 
tellement d'erreurs dans l'application des intérêts aux questions qui se rencontrent à chaque pas dans l'économie forestière, qu'il était indispensable, sinon de traiter à fond toutes ces questions, du moins de prémunir le lecteur contre des analogies présentées trop souvent comme des vérités dans certains livres forestiers. Il était indispensable aussi de rendre plus claires et plus simples les notions d'économie forestière qui vont suivre en les dégageant de toute discussion sur l'application des intérêts à la constitution des exploitations forestières. Il faut en retenir ceci : c'est que les valeurs formées par la végétation suivent une loi ascendante qui leur est propre et qui n'est pas encore connue; elles ne suivent jamais la loi de formation par l'intérêt composé à quelque taux qu'on le compose. Il en résulte qque si on représente les valeurs en croissance par la loi des intérêts composés, soit pour assigner un prix aux peuplements dans les expertises, soit pour calculer le taux du placement des fonds engagés dans une exploitation forestière, on n'arrive jamais qu'a une solution approchée, relative ou conventionnelle. On n’atteindra la solution vraie que le jour où on aura trouvé la loi mathématique de la formation de la valeur dans les arbres et les peuplements en croissance. La science du forestier consiste à s'affranchir autant que possible de cette inconnue et à donner aux choses leur veritable signification, quand la question économique à résoudre le met en face de cette inconnue et qu'il ne peut s'y soustraire. 

DEUXIÈME PARTIE

\section{CONCEPTION THÉORIQUE}

\section{L'EXPLOITATION FORESTIÈRE}





\title{
GONSTITUTION DE L'EXPLOITATION FORESTIĖRE
}

\author{
Article 1 er.
}

\section{Éléments constitutifs de l'exploitation.}

Nos 5ว. - Définition de la forêt.

56. - Éléments constitutifs de l'exploitation : l'arbre isolé et le peuplement d'un seul âge.

57. - Disposition de ces éléments dans l'exploitation forestière : trois types.

58. - Exploitations d'arbres isolés.

59. - Exploitations de peuplements d'un seul âge.

60. - Exploitations mixtes d'arbres isolés et de peuplements d'un seul âge.

61. - Sens du mot peuplement en sylviculture.

62. - Modes de perception de la récolte : trois systèmes de coupes.

63. - Trois modes d'assiette des coupes, leur importance.

$\mathrm{N}^{\circ}$ 55. - La connaissance exacte de la conception théorique de l'exploitation est indispensable à celui qui veut être producteur de bois, comme elle l'est à celui qui veut installer une entreprise quelconque, agricole ou industrielle. Il faut que l'un et l'autre connaissent à fond les organes et la constitution de leur entreprise, non pour toujours en suivre servilement les règles, mais, au moins, pour en raisonner en connaissance de cause et s'en approcher dans les limites convenables. Comment pourra-ton régler l'aménagement d'une forêt donnée à laquelle on veut faire produire du bois de 120 ans, si on ignore l'organisation de l'exploitation aménagée?

A un autre point de vue, la théorie de l'exploitation fo- 
restière permet seule d'asseoir des règles certaines pour la solution des questions les plus délicates, telles que les estimations de la propriété et de ses démembrements. Comment, par exemple, pourrait-on formuler et appliquer la règle que l'usager, absorbant tous les fruits, a droit, en cas de rachat, à la valeur des récoltes pendantes, si on ne connaît pas la constitution de l'exploitation forestière et si on ne sait pas en faire l'analyse?

La théorie seule éclaire la pratique et il est nécessaire de s'en pénétrer d'une manière didactique.

Chaque science a ses définitions par lesquelles son langage est précisé et qui servent de base aux explications ultérieures. L'économie forestière a, aussi, sa terminologie spéciale et ses généralisations théoriques; les omettre serait s'exposer à ne pas être compris, car il n'y a de science réelle qu'avec des définitions nettes et précises.

Il est indispensable d'observer que dans les sciences économiques et sociales qui empruntent leurs termes techniques au langage usuel, le même mot a souvent des sens différents, suivant la science qui s'en est emparé. Une définition n'est jamais absolue; elle ne conserve son sens et sa portée que dans l'ordre d'idées de chaque branche scientifique.

Nous rencontrons, dès le début, une application de cette remarque et nous aurons l'occasion d'en citer d'autres ultérieurement. (Voir nº 92 , futaie.)

Dans le langage juridique, la forêt est un immeuble implanté d'arbres et dont la production principale est le bois $\left.{ }^{1}\right)$.

Des arbres isolés dans les champs et sur les routes, ceux des avenues dans les domaines ruraux, ceux des

( ${ }^{2}$ On peut ajouter anssi les écorces à tan et à liège et les résines; ces produits, s'ils sont la production principale de l'immeuble boisé, en font également une forèt. 
pâturages dits prés-bois, ne constituent pas des forêts dans la science du droit.

La distinction est importante, car aux forêts seules s'applique la législation spéciale du Code forestier; les arbres des autres immeubles sont régis par le droit commun, civil ou pénal.

Pour la sylviculture, la forêt est une association d'arbres vivant ensemble sur le même terrain, un groupement d'essences sociables, ayant un seul ou plusieurs âges et dont il faut étudier les conditions de la végétation de manière à les faire vivre dans le meilleur état possible et à leur assurer le plus haut rendement.

En économie forestière, le sens du mot forêt est tout autre: c'est une exploitation rurale destinée à la production du bois.

Le mot exploitation a ici le sens de fabrication, entreprise; exploiter signifie faire valoir, conduire et diriger une production déterminée. La forêt est une usine à fabriquer du bois, s'il est permis de s'exprimer ainsi, par assimilation économique.

La technologie forestière emploie le même mot exploitation pour désigner l'abatage et la facon d'une coupe; nous ne l'emploierons point dans cette acception spéciale, car cette exploitation est pour l'économiste la réalisation de la l'écolte.

Des arbres isolés dans les champs ou dans les pâturages forment aussi une exploitation à bois; mais celle-ci est généralement accessoire à l'exploitation du domaine, c'est ce qui la différencie de la forêt, dans laquelle l'entreprise de la production du bois est le but principal de l'exploitant.

A cette production du bois, il convient de joindre celle des écorces à tan et à liège et celle de la résine. Les immeubles qui y sont consacrés (taillis à écorces du NordEst, chênes-liège de Provence et d'Algérie, pignadas des 
Landes), sont aussi des forêts dans le sens économique, mais des forêts spéciales, dont nous n'aurons pas l'occasion de nous occuper, car il faut savoir se restreindre et l'étude de l'exploitation à bois est encore assez vaste.

$\mathrm{N}^{\circ}$ 56. - Toute exploitation a ses éléments constitutifs, comme toute machine a ses organes dont il faut avant tout étudier la fonction pour se rendre compte des résultats. Les éléments de l'exploitation forestière sont:

L’arbre isolé;

Le peuplement d'un seul âge ( $\left.{ }^{1}\right)$.

L'arbre isolé est élevé pour lui-même et considéré pour son travail individuel, soit qu'il existe sur des terrains improductifs (routes, canaux), sur des cultures agricoles (champs, pâturages), ou sur des cultures forestières (taillis, etc.). Dans les peuplements provenant de semis naturels ou artificiels ou de plantations datant de la même époque, l'arbre perd en quelque sorte son individualité ; il travaille en société d'autres tiges de même âge et de même force. C'est la fabrication collective qui est, en quelque sorte, le but de l'exploitant. Si, par exemple, celui-ci abat un hectare de taillis de 30 ans et s'il en réalise 150 stères, il considère ces 150 stères comme le produit du peuplement sans s'inquiéter des tiges qui les ont fournis.

Quand nous disons que l'arbre travaille et qu'il est l'ouvrier producteur du bois, nous faisons abstraction de la réalité des choses, c'est-à-dire du sol, de l'air et de la lumière qui ont été les véritables agents producteurs, ou plutôt les causes initiales de la production.

L'agronome agit de même en disant que les betteraves produisent des racines à sucre; l'économiste n'examine que les résultats.

( $\left.{ }^{1}\right)$ Il nous arrivera souvent d'abréger et de désigner par le seul mot peuplement les massifs forestiers formés de tiges uées à la même époque. 
Il est bien certain que dans l'exploitation forestière, comme dans l'exploitation agricole, l'exploitant aura toujours égard aux règles culturales dictées par l'observation et la science; il n'y a d'utiles et fructueuses exploitations qu'à cette condition. L'économie forestière, loin de dédaigner la sylviculture, doit toujours s'inspirer de ses conseils et les suivre avant tout.

Cette observation faite, nous n'y reviendrons plus; toutefois elle était nécessaire, car l'économie forestière n'est une branche spéciale et distincte de la science forestière, qu'à la condition d'être une application de la sylviculture. Ses conceptions et ses théories seraient inefficaces si elles négligeaient ou heurtaient les indications fournies par la culture, comme dans une usine les combinaisons les plus ingénieuses ne sont pratiques que si elles s'adaptent aux données des sciences physiques ou chimiques que l'industriel veut mettre en ouvre. Il est arrivé trop souvent que des plans d'exploitation ont prescrit dans les forêts des coupes bâtardes et sans nom, ou des traitements contraires aux indications culturales; le plan d'exploitation n’a pu marcher convenablement et la forêt elle-même a été compromise.

L'étude économique de l'arbre et du peuplement d'un seul âge est la base de l'organisation et de la constitution de toute exploitation forestière. Les lois suivant lesquelles l'un et l'autre forment le volume et la valeur sont des plus intéressantes; elles sont aussi des plus délicates et des plus difficiles à exprimer par le calcul élémentaire. De nombreux écrits ont été publiés et bien des efforts ont été tentés en France et en Allemagne. Si le résultat n'a pas encore été complètement satisfaisant, c'est que la donnée expérimentale manque le plus souvent; celle-ci sera l'œuvre des stations de recherches dont la création s'est effectuée en Allemagne dès 1875, et en France en 1882. Il y a lieu d'espérer que l'étude économique de l'arbre et du 
peuplement, considérés comme organes de l'exploitation forestière, ne tardera pas à se produire ; il y a de ce côté un intéressant travail de nature à tenter la sagacité et la science des forestiers. Toutefois, l'exacte et entière connaissance des lois qui président à la formation du volume et de la valeur n'est pas indispensable pour comprendre la constitution des exploitations forestières; celles-ci ont été formées, à l'époque la plus reculée, sans la connaissance de ces lois et au moyen de combinaisons qui permettent de s'affranchir des parties encore ignorées. Ces combinaisons ne sont pas nombreuses et les types d'exploitations forestières sont assez restreints. Les tentatives qui ont été faites pour en accroître le nombre n'aboutiront à un résultat pratique que par la découverte des lois de la végétation. Si le progrès peut venir un jour de ce côté, Jes anciens modes d'exploitation, sanctionnés par l'expérience d'un long passé, suffiront, longtemps encore, à tous les besoins. Ils suffisent, en tout cas, à l'étude élémentaire que nous entreprenons.

$N^{0} 57$. - Ces organes de fabrication, ces éléments constitutifs de l'exploitation forestière, l'arbre et le peuplement d'un seul âge, se disposent et se groupent de manière à former l'exploitation suivant trois types:

Les exploitations d'arbres isolés;

Les exploitations de peuplements d'un seut âge;

Les exploitations mixtes d'arbres isolés et de peuplements d'un seul âge.

Il est impossible de concevoir d'autres combinaisons avec deux éléments.

Par là, se trouve expliqué le petit nombre de modes culturaux ou de traitements forestiers auxquels on peut soumettre un immeuble boisé.

Il est juste de faire remarquer que ces modes de traitements sont le plus souvent locaux, c'est-à-dire com- 
mandés par les exigences des essences dans le pays, suivant la station, la nature du sol ou le climat. Le traitement d'une forêt date toujours de la plus haute antiquité ; l'observation prolongée et l'expérience aequise l'ont consacré; il n'est pas possible de le changer sans une témérité que les faits viennent souvent condamner et que le succès est loin de toujours couronner.

La sagesse de l'exploitant consiste à se défendre contre ces témérités et contre des essais de changements dans le traitement local de l'immeuble boisé. Que le propriétaire se rende compte de la constitution de son exploitation, qu'il l'améliore et la fasse progresser vers une production meilleure et plus utile; le champ est assez vaste et le profit assez séduisant pour qu'il borne ses efforts à cette amélioration graduelle, sans aller jusqu'à vouloir tenter la nature, en modifiant un traitement cultural consacré par des siècles d'expérience.

No 58. - Les exploitations d'arbres isolés se rencontrent dans différentes circonstances:

A). Dans la campagne:

$1^{\circ}$ Arbres plantés sur des terrains auxquels on ne demande aucune autre production: arbres des arenues, des routes, des cananx, des grandes haies de Normandie, etc.;

$2^{\circ}$ Arbres plantés sur des terrains auxquels on demande d'autres produits, tels sont les arbres des champs et des domaines agricoles, les peuptiers des prairies, les épicéas des paturages dits prés-bois, etc.

$B)$. Dans les forêts:

$1^{\circ}$ Le jardinage;

$2^{\circ}$ Le furetage;

$3^{\circ}$ La futaie étagée.

Le jardinage est un mode de traitement ayant pour but 
de fournir des arbres isolés par la seule action de la nature et percrus au milieu d'un mélange confus de tiges de tous âges et de toutes dimensions, qui est destiné à la fois à garnir le sol et à fournir des remplaçants aux arbres de coupe. Une partie de ces tiges secondaires forme ainsi un remplissage qui protège le sol et qu'on ne coupe pas. Mais parfois, aussi, ces tiges fournissent une exploitation intermédiaire d'arbres de plus faibles dimensions, isolés naturellement de la même manière, quand le prix des produits permet de réaliser ces petits arbres en nombre déterminé par les règles de l'exploitation.

Le jardinage s'applique surtout aux essences résineuses à l'état pur ou mélangé dans les régions montagneuses ; il est fondé sur la régénération par semis naturels, et ne peut se pratiquer au plus tôt qu'à partir du moment où les arbres ont atteint l'âge de fertilité; il est susceptible de procurer des arbres de l'âge le plus avancé.

Le furetage est une variété du jardinage fondée sur la faculté que possèdent certaines essences de rejeter de souche à l'ombre. On coupe toutes les tig'es qui ont atteint une dimension déterminée $(0,25$ de tour en Chablais, 0,30 à 0,35 en Morvan) en laissant tout ce qui n'a pas cette dimension pour fournir à la fois des tiges de remplacement et un remplissage destiné à abriter le sol ( $\left.{ }^{1}\right)$.

Le furetage s'applique presque exclusivement au hêtre, essence d'ombre; on le trouve sur les flancs abrupts de certaines montagnes où il forme d'excellentes forêts de protection. On le rencontre aussi dans des régions de coteaux en des conditions de climat encore assez mal connues et qui assurent la reproduction du hêtre par souches. Le traitement étant fondé sur la faculté de rejeter, la coupe ne peut dépasser l'âge où les souches auraient

(1) Voir "Les Forèts du Morran ", par M. Cathelot, sous-inspecteur des forêts. Revue des eaux el foréts, 1876, p. 409. 
perdu le pouvoir d'émettre des rejets, c'est-à-dire, 25 à 35 ans, selon les localités.

La futaie étagée est un mode de traitement qui a été peu décrit, bien qu'il existe en plusieurs régions montagneuses, pratiqué par de petits propriétaires très soigneux de leur's forêts et désireux d'en obtenir des arbres de toutes dimensions. Il consiste à avoir sur la coupe exploitable des arbres espacés entre eux du temps qu'on met à revenir sur la même coupe, par exemple: des arbres de 30, 60, 90, 120 ans, etc., si la coupe revient tous les 30 ans. Cette coupe se fait par pieds d'arbres, en laissant tous ceux de ces divers âgres qui sont en bon état de végétation et en abattant ceux qui ne sont pas capables de prospérer jusqu'à la coupe suivante ou qui sont trop serrés, de manière à mettre les cîmes des arbres réservés en état isolé, de telle sorte que le massif se reforme dans l'intervalle d'une coupe à l'autre.

On alimente la réserve par un certain nombre de brins venus de semences à la faveur des intervalles existant entre les arbres. La coupe est, ainsi, une sorte de coupe d'ensemencement opérée avec des arbres de divers âges et de différentes dimensions.

Ce traitement est le résultat de la stricte application, en pays de montagnes et aux essences qui repoussent mal de souches, du balivage prescrit par les anciennes ordonnances $\left({ }^{1}\right)$ pour les taillis sous futaie.

On a critiqué cet ancien plan de balivage pour les taillis avec juste raison,parce qu'il détruit le taillis et transforme la forêt de taillis sous futaie en une futaie d'âges étagés où le taillis n'existe pour ainsi dire plus et où la régéné-

(1) Ord. de 1669 : Il sera laissé 16 baliveaux (32 par liectare) outre et par dessus tous les anciens et modernes par arpent ( 00 ares).

Ord. de Léopold de Lorraine, d'août 1701 : Il sera laissé sur claque arfent (20 ares) 12 baliveaux de l'àge (60 par liectare) des plus beaux brins naissants outre et par dessus les arbres de vieilles écorces, anciens et modernes des coupes précédentes. 
ration se fait par la semence; mais on n'a pas assez vu que cette prescription avait précisément pour but de sacrifier un taillis sans valeur pour lui substituer des arbres d'âges variés dont les produits sont plus utiles et plus précieux. En tous cas, dans les terrains de montagnes et dans les forêts peuplées de hêtres repoussant mal de souches, ce traitement est très utile et très productif; il est pratiqué avec avantage sur le grès vosgien et le grès bigarré des petites montagnes des Vosges où les forêts de hêtres sont exposées à être détruites par l'envahissement des plantes silicicoles (myrtilles, bruyères, genêts) causé par les coupes claires des taillis sous futaie; il rend aussi de très utiles services dans les forêts de hètres mélangées de sapins des mêmes régions ( $\left.{ }^{1}\right)$; il permet enfin de produire des grands arbres d'âge avancé ainsi que d'autres de moindre taille avec de petites contenances, puisque 30 hectares permettent d'obtenir chaque année un hectare de coupe livrant au revenu des artres de 30,60, 90 ei 120 ans, par exemple.

Ce traitement méritait d'être distingué par un nom spécial $\left({ }^{2}\right)$, car il diffère du jardinage en ce que l'on nettoie le sol, à chaque coupe, du remplissage formé par les basses tiges et parce qu'il n'offre pas le mélang'e cle tous âges qui le caractérise. Il existe, il est vrai, des jardinages à rotation de 10 ou 20 ans, où les arbres tendent à s'étager de 10 en 10 ans ou de 20 en 20 ans; mais, même dans ce cas, il y a toujours un remplissage non utilisé qui n'est jamais coupé, tandis qu'ici tout le sous-bois est abattu lors de la coupe.

Il diffère également du taillis sous futaie, puisqu'il se

(') Voir M. Guyot, Histoire d'un domaine rural, Nancy, 1886.

${ }^{2}$ ) Ce mode de traitement a été appelé futaie batarde par des auteurs qui ont préconisé, surtout et partout, la futaie pleine résultant de peuplements d'un seul âge et exploitée à long terme : ce mot malheureux, par le blâme implicite qui y était contenu, a fait détruire, en France, plus d'arbres qu'on ne saurait croire. 
repeuple presque uniquement par la semence; le taillis de rejets n'y existe pour ainsi dire point et il s'adapte.aux forêts de hêtre mélangées de sapins qui ne supportent pas le régime des taillis.

$\mathrm{N}^{\circ}$ 59. - Les exploitations de peuplements d'un seul âge se r'encontrent dans les forêts suivantes :

$1^{\circ}$ Les taillis ordinaires peuplés d'essences feuillues susceptibles de se reproduire par rejets. La coupe se fait à tous les âges compris entre celui de la première utilisation du bois et celui où les souches perdent la faculté de rejeter;

$2^{\circ}$ Les taillis sartés, qui ne sont que des taillis ordinaires, dont la coupe est suivie d'une culture en céréales facilitée par l'écobuage des ramilles et des résidus de l'exploitation forestière. On les rencontre dans les Ardennes et dans certaines régions montagneuses à sol siliceux où le chêne végète avec vigueur ;

$3^{\circ}$ Les pineraies de Champagne, de Sologne et du Centre de la France, où le peuplement est coupé sur des contenances repeuplées ensuite par plantations ou semis artificiels;

$4^{\circ}$ Les sapineraies ( $\left.{ }^{1}\right)$, provenant de plantations ou de semis de rnain d'homme en épicéas dans le Nord de la France, en Luxembourg et en Belgique, et exploitées comme les pineraies à un âge inférieur à celui de la fertilité;

$5^{\circ}$ Les bandes d'épicéas des régions montagneuses du Jura et des Alpes suisses, qu'on exploite par contenances égales, longues et étroites, se suivant de proche en proche et sur lesquelles on coupe tout le peuplement en laissant à la nature le soin de repeupler le terrain par les semen-

( $\left.{ }^{1}\right)$ Nous empruntons le mol lelge pour caractériser ces forêts de création artificielle et les distinguer des sapinières, forêts naturelles de sapins traitées en futaie à un âge qui dépasse celui de la fertilité des arbres. 
ces du massif voisin. Certaines forêts de pins maritimes s'exploitent de cette manière dans le Midi de la France, sans que la coupe ait besoin d'être faite par bandes, car les cônes des arbres abattus, abandomnés sur le sol, suffisent à le repeupler (forêts des mines de la Grande-Combe, Gard). Ces forêts ne peuvent naturellement s'exploiter qu'à un âge postérieur à celui de la fertilité des arbres;

$6^{\circ}$ Les forêts de fitaie pleine, résineuses, feuillues ou d'essences mélangées, exploitées suivant la méthode dite du réensemencement naturel et des éclaircies. La coupe du peuplement s'y opère sur des contenances dans lesquelles on laisse temporairement des arbres porte-graines dans le but de produire et abriter les semis. Ces arbres diminuent de nombre à mesure que le semis grandit (coupes secondaires) et finissent par disparaître (coupes définitives). Ces forêts, dont la reproduction est toute naturelle, sont exploitées à des âges avancés, exigent une grande contenance et existent dans le Centre et le Nord de la France pour le chêne et le hêtre, ou dans certaines montagnes pour le hêtre et le sapin.

$\mathrm{N}^{\circ}$ 60. - Les exploitations mixtes d'arbres isolés et de peuplements d'un seul âge sont formées par deux forêts en quelque sorte superposées sur le même terrain, l'une en arbres isolés par'la main de l'homme, l'autre en peuplements d'un seul âge venus dans les intervalles laissés libres par les arbres. On les rencontre dans:

les taillis sous futaie simple ou composée,

les forêts de peuplements avec arbres de réserve.

$I^{\circ}$ Les forêts de taillis sous futaie occupent les régions de plaine et de coteaux de toute la France. L'exploitation d'arbres choisis et isolés est formée par des tiges de différents âges, recevant différents noms suivant leur catégorie (bativeaux, modernes, anciens, bis-anciens). Celle de peuplements est formée par un taillis ordinaire vivant 
entre ces arbres, et formant un remplissage protecteur du sol mais qui, à la différence des forêts jardinées, se coupe quand l'âg'e adopté est arrivé. La récolte comprend ainsi à la fois du taillis de cet âge (30 ans, par exemple) et des arbres ayant deux fois cet âge (modernes de 60 ans) ou trois fois cet âge (anciens de 90 ans), etc..., selon qu'on a conservé sur les coupes différentes catégories d'âges.

La futaie sur taillis est simple quand on ne réserve qu'une classe d'âge et qu'on ne coupe ainsi que des modernes qui sont remplacés par des baliveaux de l'âge du taillis en nombre égal. Ces forêts se rencontrent en assez grande quantité dans le Centre de la France, surtout dans les domaines des particuliers. La futaie est, au contraire, composée quand la récolte est formée par un certain nombre d'arbres de différentes catégories, modernes, anciens et bis-anciens; c'est-à-dire quand on coupe à la fois des arbres de 60, de 90 et de 120 ans, si l'âge du taillis est de 30 ans. Le traitement en taillis sous futaie composée est essentiellement français, c'est celui de presque toutes nos foréts des régions de collines et de coteaux.

Dans ces forêts, la régénération se fait à la fois par les rejets des souches et par les semences des arbres. Le peuplement se renouvelle par rejets, les arbres se recrutent par des brins de souche ou semis pris dans le taillis (balireaux de 30 ans). Les bativeaux sont ainsi des arbres de remplacement et jamais des arbres de coupe.

Les arbres des taillis sous futaie forment une exploitation plus perfectionnée que ceux du jardinage; ceux-ci doivent leur origine à la seule sélection naturelle, tandis que ceux des futaies sur taillis sont choisis et isolés par la main de l'homme. Le remplissage dû au taillis protège le sol, mais assure une supériorité à l'exploitation en ce qu'il est utilisé et coupé à des époques fixes; tandis que dans le jardinage, il reste permanent et sans utilisation autre que de garnir et de protéger le terrain. Ces deux 
exploitations superposées forment des forêts dont la constitution est la plus compliquée, mais aussi la plus intéressante et la plus digne de l'attention du forestier;

$2^{\circ}$ On trouve les forêts de peuplements avec arbres de réserve dans les futaies feuillues dont l'ordonnance de 1669 nous a indiqué le type en prescrivant $\left({ }^{1}\right)$ qu'à la coupe " on réserverait 10 chênes des plus vifs et de la plus belle ( venue par arpent " (20 arbres par hectare).

On rencontre aussi cette exploitation mixte dans certaines pineraies où on réserve sur la coupe un certain nombre de beaux arbres destinés à parcourir deux fois l'âge du peuplement et à fournir des produits de plus forte dimension.

Ces arbres choisis et isolés par l'exploitant forment une réserve simple, en ce sens qu'on les abattous quand ils ont deux fois l'âge du peuplement en les remplaçant par un même nombre de tiges choisies dans le peuplement. Il y a, comme dans les taillis sous futaie simple, une exploitation d'arbres superposée à celle du peuplement, ceux-ci se coupant en même temps que lui.

Le but de ces exploitations mixtes est de fournir des bois de plusieurs dimensions et ayant des degrés différents d'utilité, sans augmenter la contenance du terrain consacrée à la forêt de peuplements.

$\mathrm{N}^{\circ}$ 61. - La sylviculture ne considère pas les forêts au même point de vue que l'économie forestière; on le concoit facilement, puisqu'elle ne s'occupe que des soins à leur donner pour les faire vivre en le meilleur état possible.

Pour elle, toute association d'arbres est un peuplement; elle distingue ainsi :

1 $^{\circ}$ Les peuplements d'un seu âge (taillis ordinaires, pineraies, futaies pleines);

(1) Ord. de 1669, titre XV, art. 11. 
$2^{\circ}$ Les peuplements de tous âges confusément métangés (forêts jardinées et furetées);

$3^{\circ}$ Les peuplements d'âges méthodiquement disposés ou peuplements à arbres de réserve (taillis sous futaie, forêts à réserves, futaies étagées).

Dans chacune de ces catégories, les peuplements peuvent être réguliers ou irréguliers, purs ou mélangés, complets ou incomplets, etc. C'est un aspect tout différent des connaissances que le forestier doit posséder et qui est dirigé uniquement vers le but cultural. Il n'était pas inutile de l'indiquer, afin qu'il ne soit pas fait confusion avec l'étude purement économique de l'exploitation.

$\mathrm{N}^{\circ}$ 62. - Dans les exploitations forestières, la récolte se percoit par des coupes dont les dispositions sont en harmonie avec la nature de l'exploitation et forment trois types:

\section{La coupe à blanc estoc; La coupe en jardinant; \\ La coupe à tire-aire.}

La première abat toutes les tiges qui se trouvent sur la contenance désignée pour former la récolte.

La seconde consiste à couper çà et là, sur la contenance affectée à la récolte, un certain nombre d'arbres, mais en laissant toutes les autres tiges.

La coupe à tire-aire consiste à abattre et à nettoyer toute la surface du terrain consacré à la récolte, en laissant un certain nombre d'arbres; c'est le blanc estoc, sauf les arbres réservés.

La coupe à blanc estoc s'applique aux taillis ordinaires, aux pineraies et sapineraies, et à toutes les forêts de peuplements sans arbres de réserve.

La coupe en jardinant s'opère dans les forêts jardinées et furetées.

La coupe à tire-aire se pratique dans les taillis sous 
futaie et dans les forêts de peuplements à arbres de réserve.

Ces mots sont très anciens et se trouvent en usage pour ainsi dire dès les débuts de l'art forestier.

L'origine des deux premiers termes est très simple. Le blanc estoc provient de ce que la coupe apparaît par la blancheur des souches (estocs), des tiges récemment abattues. Dans la coupe en jardinant, le forestier opère comme le jardinier qui choisit les meilleurs légumes de ses planches.

L'étymologie du tire-aire est rendue plus difficile par une altération qui parait remonter à l'ordonnance de 1669 , où l'on écrivait tire et aire et que certains auteurs ont aggravée en parlant de coupes à tire et $\dot{a}$ aire. Le mot que nous employons avec Littré et Dralet $\left({ }^{1}\right)$ se trouve dans les actes les plus anciens; il signifie que le sol (l'aire) se tire, apparait nettement entre les arbres laissés sur pied. Le vieux mot français, qui existe encore dans un certain nombre d'expressions, se rencontre aussi dans l'espagnol tir $a$, signifiant bande, et montre qu'on a voulu désigner des parties sinueuses de sol entre les arbres.

Quand on a prétendu que cóuper à tire-aire signifiait asseoir les coupes en suivant, de proche en proche et sans intermission d'une année à l'autre $\left({ }^{2}\right)$, on a commis une erreur, car toutes les coupes de toute nature et dans tous les modes de traitement s'effectuent de proche en proche.

On remarque, enfin, dans tous les actes anciens et dans toutes les vieilles ordonnances, que la coupe à tire-aire est toujours opposée à la coupe en jardinant; cette opposition montre qu'il s'agit d'un mode de réalisation de la récolte et non d'un traitement spécial des forèts. Nulle part, on ne voit le mot tire-aire appliqué à un régime ou mode cultural. Les coupes pratiquées à tire-aire s'appliquent aussi bien aux taillis sous futaie qu'aux futaies avec réserves décrites dans l'ordonnance de 1669 et à toutes les forêts dans lesquelles on garde plus ou moins d'arbres. On a donc eu tort de donner ce nom au mode cultural résultant uniquement des réserves prescrites par l'ordonnance de 1669, car le traitement résultant de la coupe n'aurait jamais été limité à ce point et à ce seul cas, et encore

(1) Dralet, Traité des foréts d'arbres résinevxx, p. 96.

$\left({ }^{2}\right)$ Voir Dictionnaires de Chailland, de Baudrillard, etc. 
aujourd'hui, le cahier des charges de l'administration des forêts dispose que les coupes de taillis sous futaie seront exploitées ì tire-aire. Le mot s'applique donc bien à un mode de coupe et non à un mode de traitement des forêts.

$\mathrm{N}^{\circ}$ 63. - A ces trois modes de coupes correspondent des méthodes particulières d'assiette et de marque, c'està-dire des mesures de gestion consacrées également par la tradition la plus ancienne:

\section{La simple assiette; \\ La marque en réserve; \\ La marque en délivrance.}

Dans les coupes à blanc estoc, on se borne à limiter la contenance par des lignes de pieds corniers (arbres d'angles) et de parois (arbres de faces), tout le reste est abandonné à la hache.

Pour les coupes à tire-aire, outre ce système de limites, les arbres à conserver sont balivés, c'est-à-dire marqués à la racine d'une ou de deux empreintes du marteau selon leur grosseur, de telle sorte qu'on puisse les retrouver en nombre et en qualité, quand la coupe sera libre; l'adjudicataire est ainsi comptable des arbres dont le propriétaire a fait le balivage $\left({ }^{1}\right)$.

Pour les coupes en jardinant, la limitation de l'enceinte n'est qu'une indication de l'emplacement où l'acquéreur trouvera les arbres qui lui sont vendus. Ces arbres sont martelés, à la racine et au corps, d'une empreinte de marteau; l'acquéreur doit représenter, après la vidange, les souches garnies de cette empreinte intacte. Les arbres dont le martelage a été fait, sont seuls vendus et délivrés; la vérification (récolement) consiste à examiner si, dans l'enceinte de la coupe, il n'y a que des souches garnies

(1) Les mots balivean, baliver ont un radical commun avec bailli et bailler qui signifie gérer, conserver, administrer. Les baillis (balivi) ont été probablement chargés de marquer les arbres daus les forèts avant l'institution des maîtrises. 
d'empreintes et aucune sans cette marque de délivrance.

Le système fort simple de ces trois modes d'assiette remonte à la plus haute antiquité. C'est dire qu'il est consacré par l'expérience et qu'on ne saurait le modifier par d'autres pratiques soi-disant perfectionnées, sans danger pour la sécurité du propriétaire. Les procédés simples sont toujours les meilleurs et tant que le commerce des bois sera en même temps cliargé de l'exploitation des coupes, le propriétaire ne trouvera pas meilleure garantie, ni plus grande sûreté que dans ces modes d'assiette traditionnels. C'est à peine si le propriétaire exploitant lui-même ses coupes et vendant leur's produits directement, pourra se relâcher de leur stricte observation: il aurait peut-être à compter avec ses bûcherons intéressés par leur travail à abattre un arbre plutôt qu'un autre et à compromettre ainsi le bon état des peuplements.

L'assiette d'une coupe, son balivage et son martelage sont les opérations capitales de l'exploitation d'une forêt. Par la coupe, en effet, le mode de traitement de la forêt s'établit et se conserve; l'entreprise forestière se maintient dans les conditions économiques que le propriétaire a voulu lui assigner. Confiée à des mains subalternes, la marque des coupes peut compromettre les bases de l'exploitation en très pelı d'années; le propriétaire livre, en réalité, son entreprise à la merci d'autrui; il n'y a plus d'exploitation raisonnée dans le sens économique du mot; le désordre s'y introduit et la pauvreté se substitue vite à la richesse. 
Article 2.

\section{Capital d'exploitation.}

$\S 1^{\mathrm{er}} .-$ Distinctions entre les exploitations.

Nos 64. - Constitution de l'exploitation forestière : travail, agents naturels, capital.

65. - Différentes espèces d'exploitations : périodique, aménagée, gradtiée.

66. - Exploitations simples et composées.

$N^{\circ}$ 64. - L'économie politique enseigne qu'on ne peut rien produire sans le concours de trois éléments : le travait, les agents naturels et le capital. Nous devons donc examiner à ce point de vue la constitution de l'exploitation forestière.

Le travail, qui est le grand facteur de toutes les entreprises agricoles et industrielles, n'intervient pas dans la fabrication du bois. C'est là, sans contredit, la grande supériorité et la note caractéristique des exploitations forestières. Dans les forêts soit naturelles, soit artificielles, c'est-à-dire semées ou plantées de main d'homme, le travail n'intervient dans l'exploitation que pour la réalisation de la récolte, car même dans ces dernières forêts, le travail n'existe qu'à titre de première mise de fonds ou de capital de premier établissement. La dépense ultérieure de plantation n'est pas un déboursé à faire à chaque période de l'exploitation et dont il faut posséder par avance le capital correspondant. Cette dépense est une simple réduction du produit de l'exploitation. Si, par exemple, une plantation de 100 francs produit 1,000 francs à 30 ans, le revenu périodiquement constant n'est que de 900 francs. C'est à ce titre que la dépense de plantation est un capi- 
tal, une fois déboursé mais toujours entretenu, dont on garnit l'exploitation.

Les agents naturels, air, lumière, humidité, etc., sont les véritables artisans de la production forestière. Comme ils sont toujours abonclants et gratuits, l'exploitant n'a jamais à s'en préoccuper autrement que pour diriger leur action au point de vue cultural.

Le capital engagé se compose de deux parties:

Le sol;

Le capital d'exploitation.

Le sol,support de toute entreprise, est un véritable capital, puisqu'on ne l'acquiert qu'en échange d'un capital argent. Le sol des forêts n'est pas un terrain vierge : il a acquis par l'effet prolongé de la végétation une certaine couche de débris organiques, racines, brindilles, humus, tout un ensemble de qualités que les gens de la campagne désignent sous le nom de terre à bois et qui est l'analogue de la couche arable dans les terres cultivées. Celle-ci provient d'un long travail accumulé, d'une réserve d'engrais non consommés et d'un ameublissement dû à des cultures prolongées. La couche arable et la qualité de terre-à-bois sont donc des épargnes antérieures, mais tellement incorporées au sol que si on doit les distinguer du terrain vierge, pour l'analyse des choses, on ne saurait les disjoindre pour évaluer la valeur vénale du terrain, ni y voir l'outillage de l'exploitation, c'est-à-dire les objets mobilisables dont on a doté celle-ci pour assurer la reproduction.

Le capital ou matériel d'exploitation est précisément cet outillage indispensable à toute entreprise; il se compose de deux parties:

Le matériel ordinaire;

Le matériel-bois.

Nous rangeons dans le matériel ordinaire celui qui est commun à toutes les exploitations de la terre: chemins 
d'accès et de vidange, maisons de gardes, places de dépôts; scieries, attelages et voitures. L'avenir y ajoutera les tramways, les chemins de fer portatifs, les câbles aériens, les plans inclinés automoteur's, etc. Nous ne faisons qu'indiquer ici la nature de ce matériel d'exploitation pour n'avoir plus à y revenir et pour nous occuper exclusivement du matériel-bois, qu'il est indispensable de bien distinguer pour se rendre compte de la constitution d'une exploitation forestière.

Ce matériel spécial, c'est la graine que l'homme ou la nature met en terre, le semis des futaies, l'ensouchement des taillis; ce sont des arbres de réserve, des peuplements et des bois sur pied. Comme tous les capitaux, ce matériel est dû, soit à un déboursé initial, soit à une économie antérieure, c'est-à-dire à des produits non consommés et mis en réserve pour servir à une production ultérieure.

Dans une forêt, on ne voit trop souvent qu'une masse d'arbres et de feuillages; il est intéressant d'étudier l'ordre et l'arrangement qui en font une entreprise de production $\mathrm{du}$ bois et d'examiner surtout comment l'épargne d'une certaine quantité de bois peut améliorer cette entreprise. Nous devons ainsi distinguer les différentes espèces d'exploitations forestières pour en déterminer le capital d'exploitation, c'est-à-dire pour acquérir la notion sans laquelle on ne peut se rendre compte de la forêt et on s'expose à la compromettre ou même à la dégrader.

$N^{\circ}$ 65. - Les exploitations de bois présentent avec les exploitations de céréales ou de plantes fourragères une différence caractéristique qui forme la difficulté de leur théorie, Les produits des exploitations agricoles se recueillent chaque année; jamais ils ne peuvent s'accumuler sur le sol. Les exploitations forestières ont, au 
contraire, des produits susceptibles de s'accumuler presque indéfiniment sur le terrain. Quand on les recueille, on percoit à la fois le produit de l'année et celui des nombreuses épargnes annuelles qui lui sont réunies, pour continuer ainsi de la même manière et à intervalles de temps égaux.

L'exploitation forestière est de sa nature une exploitation périodique, qu'elle soit formée d'arbres ou de peuplements .

Un terrain planté de peuplíers qu'on coupe à 30 ans pour les remplacer après la coupe par des tiges de 1 an, forme une exp'oitation d'arbres isolés à produit périodique.

Un hectare de taillis qu'on coupe tous les 30 ans, forme une exploitation périodique de peuplements d'un seul âge.

Ainsi, l'arbre et le peuplement, en ne livrant pas tous les ans leur produit, en accumulant leur production annuelle, sont des réserves, des sortes de greniers conservant des économies annuelles et les faisant fructifier, bien plutôt que des instruments de revenu, pour les besoins de chaque année; ceci est à la fois un avantage et un inconvénient.

L'avantage vient de ce que l'arbre et le peuplement sont de merveilleux instruments pour créer un capital et augmenter une fortune. L'inconvénient naît de ce que, considérés en eux-mêmes, ils ne donnent pas un revenu pour les besoins annuels de la vie. Cet inconvénient n'est qu'apparent, car on peut, par des combinaisons, par des groupements d'arbres et de peuplements, transformer la nature périodique de l'immeuble en une exploitation à produits annuels.

Cette transformation d'un immeuble à produits naturellement périodiques en un immeuble à produits annuels forme l'aménagement ou l'exploitation aménagée, mot dont l'étymologie (ad menagium) indique l'adaptation aux besoins du ménage, besoins essentiellement annuels 
(Proudhon, Traité de l'usufruit) et en manifeste bien la nature et le caractère $\left({ }^{1}\right)$.

30 peupliers de 30 ans, exploités tous les 30 ans, forment l'exploitation périodique d'arbres isolés. - 30 peupliers âgés de 1 à 30 ans, dont on exploite chaque année l'arbre de 30 ans, en le remplacant par une tige de 1 an, forment l'exploitation aménagée.

30 hectares de taillis, coupés tous les 30 ans, sont une exploitation périodique de peuplements. - 30 hectares de taillis, âgés de 1 à 30 ans, sont une exploitation aménagée, car si on coupe l'hectare de 30 ans, celui de 29 ans le remplacera pour livrer l'année suivante un lectare de 30 ans et on aura indéfiniment un revenu annuel constant qui sera l'hectare de 30 ans.

Ces exemples suffisent pour faire comprendre la double nature des exploitations forestières; mais entre ces deux genres d'exploitations il y a place pour une espèce intermédiaire qu'on peut appeler exploitation graduée, parce que les âges se suivant comme en gradins, les produits se perçoivent à intervalles égaux, supérieurs à une année et inférieurs à l'âge de la coupe.

30 hectares de taillis ordinaires peuvent ètre divisés en dix coupons de 3 hectares chacun pour fournir une coupe triennale de 3 hectares. La forèt présente :

3 hectares de 1 an
3 hectares de 3 ans
3 hectares de 6 ans
3 hectares de 9 ans
$\ldots \ldots \ldots \ldots \ldots \ldots$

Nous aurons ainsi à étudier la manière dont sont constituées ces trois sortes d'exploitations :

Exploitation périodique;

Exploitation aménagée ;

Exploitation graduée.

Mais avant d'entreprendre cette étude, il importe de

(') Voir Note $B$. 
remarquer que toute exploitation aménagée est constituée par des éléments à exploitation périodique. Les exemples précédents montrent que l'hectare de coupe, celui qui a 30 ans, ne reviendra en tour de coupe que tous les 30 ans. Il en est de même de chaque hectare dans la suite aménagée à 30 ans et la même formation existe dans l'exploitation graduée. C'est donc l'exploitation périodique qui est la base de toute entreprise forestière : c'est par elle que l'étude doit débuter.

No 66. - L'exploitation, soit périodique, soit aménagée ou graduée, peut être simple ou composée.

Elle est simple quand l'exploitant, n'a d'autre but que de fournir des bois d'un seul âge et, par suite, d'une seule dimension. C'est, par exemple, le taillis exploité à 25 ans : chaque année, il fournit des bois de la seule dimension et qualité correspondant à cet âge.

L'exploitation est composée quand elle est organisée de manière à fournir des bois de divers âges. Outre les bois de l'âge le plus avancé, appelés produits principaux, on recueille des bois d'âge inférieur dits produits intermédiaires. Par exemple, une futaie pleine, exploitée à 120 ans, fournit du bois de cet âge, mais, en outre, des bois de 40,60, 80, 100 ans, par les éclaircies faites à ces âges. La futaie élevée sur un taillis de 30 ans produit à chaque coupe des arbres de 60 ans (modernes), de 90 ans (anciens) et de 120 ans (bis-anciens). On conçoit que les exploitations composées rendent plus de services à la consommation en lui livrant du bois de dimensions variées. Dans chaque mode de traitement on peut presque toujours organiser des exploitations composées. Le matériel étant naturellement plus compliqué que celui des exploitations simples, nous examinerons d'abord celles-ci.

Dans cette étude nous rencontrerons des exploitations mixtes d'arbres et de peuplements produisant, comme les 
exploitations composées, des tiges de deux âges et offrant, par conséquent, en partic, les mêmes avantages. Toute. fois, ce n'est pas le même ordre d'idées et ce ne sont pas les mêmes produits. L'exploitation mixte fournit des bois d'arbres isolés et des bois de peuplements; ce sont deux exploitations superposées qui peuvent étre chacune simple ou composée, ou l'une simple et l'autre composée. Un taillis sous futaie peut fournir du bois de 30 ans provenant du peuplement et des arbres de 60,90 et 120 ans venant de la futaie. C'est une exploitation mixte, simple pour le taillis, composée pour la futaie.

Il est évident que, dans l'analyse économique, l'exploitation composée doit être considérée comme formée d'exploitations simples, en quelque sorte juxtaposées.

\section{\$2. - Exploitations simples.}

Nos 67. -- Exploitations périodiques d'arbres, matériel-bois.
68. - - - - de peuplements d'un seul âge.
69. - - - - mixtes d'arbres et de peuplements.
70. - Exploitations aménagées d'arbres isolés.
71. - - - $\quad$ - de peuplements.
72. - - - - mixtes d'arbres et de peuplements.
73. - Exploitations graduées : leur constitution et leur matériel.
74. - Utilité de ces exploitations pour les petites forêts.
7\%. - Combinaisons des forêts jardinées pour l'aménagement des grands domaines.
76. - - _ pour les domaines moyens.

NN 67. - Exploitations périodiques. - Dans ces exploitations d'arbres isolés, tels que les arbres des champs et des foréts jardinées, le matériel-bois est réduit à sa plus simple expression; c'est le plant initial, naturellement venu ou artificiellement mis en terre. La valeur en est ainsi très minime, ce qui est très heureux, parce qu'elle rend rémunérateur le taux de placement du capital engagé dans l'exploitation. Une plantation faite à grands 
frais, comme celle des arbres que la ville de Paris destine à l'ornement de ses voies publiques et qu'elle renouvelle tous les 25 ou 30 ans, serait une exploitation périodique ruineuse pour celui qui l'entreprendrait dans le but unique de produire du bois.

Les forêts jardinées sont, de leur nature, des exploitations aménagées, en ce sens que les familles d'arbres qui constituent la forêt, depuis le brin naissant jusqu'à la tige exploitable, permettent de livrer chaque année à la récolte un certain nombre d'arbres faits. La nature crée donc des exploitations à produits annuels; mais si on considère un arbre isolément, ayant par exemple 100 ans, et si on regarde le produit comme revenant tous les 100 ans, il faut bien lui assigner, pour matériel d'exploitation, la valeur du jeune semis qui lui a donné naissance. A vrai dire, l'exploitation périodique n'existe pas dans les forêts jardinées. Il suffit, pour s'en convaincre, de considérer la manière dont ces forêts se sont constituées: sur un terrain nu, le vent a apporté des graines à différentes époques et en différentes quantités; les arbres en provenant en ont émis à leur tour; puis, l'homme intervenant a coupé les tiges dont les dimensions convenaient à ses besoins, respectant les plus petites pour remplacer les arbres abattus. Qu'on rase à blanc estoc un état ainsi constitué et on verra qu'il faudra un temps très long pour le reproduire, temps indéterminé qui n'est pas celui d'un retour régulièrement périodique comme on peut le concevoir pour une exploitation raisonnée.

$\mathrm{N}^{\circ} 68$. - Dans les exploitations périodiques de peuplements d'un seul âge, le matériel-bois est formé d'une manière trèssimple.Si on considère un taillis ordinaire, on voit que l'ensouchement est l'élément continuellement reproducteur du taillis, tous les 30 ans, par exemple, quand l'exploitation est réglée à 30 ans. Cet ensouchement n'est 
pas l'œuvre d'un jour; il est acquis par une attente assez longue due à l'action de la régétation. Il ne se confond pas avec la couche de terre à bois qui garnit le sol vierge, car il peut être séparé du sol par l'extraction des souches et a bien le double caractère de tout capital affecté à une entreprise, qui est de provenir d'une épargne antérieure et d'être mobilisable.

Dans les forêts de peuplements d'un seul âge qui se régénèrent par semis artificiels ou naturels (pineraies, sapineraies, futaies pleines), ce semis constitue, comme l'ensouchement, le matériel-bois de l'exploitation. Nous avons vu ( $\left.n^{\circ} 66\right)$ que s'il provient d'un travail effectué de main d'homme, la dépense est une mise de premier établissement ne se présentant qu'une seule fois, car aux coupes ultérieures, elle forme seulement un prélèvement sur la récolte, de manière à renouveler le matériel initial de l'exploitation.

Nous verrons, au n $n^{\circ} 127$, la manière dont le semis et l'ensouchement doivent êtreévalués quand il s'agira d'assigner une valeur au capital engagé clans l'exploitation, pour comparer le revenu au capital et en déduire le taux du placement.

Lorsqu'on examine une exploitation périodique de peuplements, par exemple un taillis ordinaire, avant l'âge de sa coupe, on remarque que le taillis grandit d'année en année en volume et en valeur jusqu'au moment où il atteint le volume et la valeur de la coupe. Cet état intermédiaire entre l'ensouchement (matériel-bois) et le taillis mûr (récolte) est ce qu'on appelle la récolte pendante. Les figures 1 et 2 représentent le diagramme de l'exploitation en taillis ordinaire au début et à la coupe mûre. La récolte pendante occupe toutes les situations intermédiaires entre ces deux états. Il en est de même dans l'exploitation périodique d'arbres isolés; pendant tout le temps qui s'écoule entre le plant qui lui a donné naissance et le 
moment où on l'abat, l'arbre est une récolte pendante dont le caractère juridique est d'être un immeuble toujours mobilisable (C. civ. 521).

$\mathrm{N}^{\circ}$ 69. - Dans les exploitations où l'arbre et le peuplement forment les deuxé léments combinés, le matérielbois est plus considérable. Il est formé de l'ensouchement et des semis qui sont le matériel de l'exploitation à peuplements d'un seul âge et, en outre, d'arbres destinés à parcourir une seconde fois le terme de l'exploitation.

Voici une exploitation de pins réglée à 50 ans et qui fournit à la fois un peuplement de 50 ans et 40 pins de 100 ans à chaque coupe périodique de 50 ans.

Le matériel d'exploitation est formé : $1^{\circ}$ par le semis ou la plantation initiale sur toute la surface; $2^{\circ}$ par 40 pins de 50 ans laissés sur pied au moment de la coupe (voir fig. $\tilde{3}$ ).

Dans 50 ans, l'exploitation sera représentée par le diagramme de la figure 4. Le peuplement aura grandi, les 40 pins auront grossi et grandi; on coupera le peuplement de 50 ans et les 40 pins de 100 ans représentés par les parties blanches de la figure 4; on réservera sur la coupe 40 pins de choix, âgés de 50 ans et pris dans le peuplement, et à chaque période de 50 ans, le produit se réalisera de la mème manière et dans les mêmes conditions.

Le semis et les arbres réservés sur la coupe forment bien l'outillage générateur de l'exploitation; leur ensemble constitue le matériel-bois.

Il semble, au premier abord, que du moment où l'exploitation esticonstituée par deux éléments, les arbres et le peuplement, le matériel-bois de cette exploitation mixte devra être composé des matériels particuliers à chacun de ces éléments tels qu'ils ont été indiqués $\mathrm{n}^{0 \mathrm{~s}} 66$ et 67 .

Il est bien certain que le pin de 100 ans qui est le résultat de notre exploitation a débuté par un semis initial, mais ce n'est pas de ce début qu'il s'agit : on doit considérer non l'exploitation faite à 100 ans, mais celle qui est installée et réglée à 50 ans. Pour monter cette exploitation, le 
propriétaire s'est imposé un sacrifice, une épargne de 40 pins de 50 ans; cette épargne est, dès lors, le capital, l'outillage affecté à l'exploitation telle qu'elle est actuellement installée et telle qu'elle fonctionnera dans la suite.

La récolte pendante est représentée par tous les états de croissance du peuplement et des arbres, intermédiaires entre le matériel-bois de la figure 3 et la coupe mûre de la figure 4.

Le matériel se rajeunit et se rétablit à l'état initial par la coupe des arbres qu'on remplace en pareil nombre pris dans le peuplement. Ces arbres de remplacement s'appellent bativeaux, et le matériel-bois de l'exploitation d'arbres superposés à celle du peuplement prend le nom de bativage.

Il y a des taillis sous futaie simple dont le matériel est formé de cette manière : ce sont ceux dans lesquels on ne laisse après la coupe que des baliveaux de l'âge du taillis. A la coupe suivante, on les trouve à l'état de modernes, c'est-à-dire avec le double d'âge; on abat tous ces modernes et on les remplace par un égal nombre de baliveaux pris dans le peuplement. Ces taillis dont la futaie est ainsi simple, c'est-à-dire d'un seul âge de coupe, existent dans un grand nombre de localités de l'Ouest et du Centre de la France.

On concoit l'avantage de ces exploitations mixtes d'arbres et de peuplements : le propriétaire réunit le bénéfice du travail isolé et de la fabrication collective du bois par l'arbre et par le peuplement.

$\mathrm{N}^{\circ}$ 70. - Exploitations aménagées. - Nous avons vu ( $\left.n^{\circ} 65\right)$ que l'exploitation aménag'ée est celle qui donne des produits annuels égaux et constants. Sa note caractéristique est un revenu annuel, quand, bien entendu, le sol et les essences sont les mêmes sur toute l'étendue de la forêt. L'aménagement est une combinaison qui a pour 
but de transformer un bien dont le produit est de sa nature périodique en un immeuble à revenu annuel égal et constant (').

Une forêt aménagée ou, en d'autres termes, une exploitation aménagée est donc celle qui présente une combinaison d'arbres ou de peuplements telle, que le produit devient annuel et sensiblement égal d'une année à l'autre.

On connaît l'effet de l'augmentation du capital engagé dans une entreprise ; il accroît la quantité des produits ou il en rapproche la jouissance à des intervalles plus courts et plus fréquents.

Le même phénomène doit se passer dans l'exploitation forestière. Si nous voulons obtenir des arbres de 120 ans, non pas tous les 120 ans, mais tous les ans, il faudra doter l'exploitation d'un capital considérable et acquérir ce capital par une économie d'au moins 120 ans. La forêt devra présenter une suite de 120 tiges d'âges gradués de 1 à 120 ans; le matériel-bois sera la suite de 119 tiges de 1 à 119 ans et la $120^{\circ}$ tige fournira le revenu.

On voit que le matériel d'exploitation est bien ici, comme l'enseigne l'économie politique, l'outillage dont on garnit le sol pour arriver à une production annuelle, outillage dû à une épargne longtemps prolongée.

Comme exemple, nous citerons 30 peupliers du Canada, âgés de 1 à 30 ans, c'est-à-dire :

$$
\begin{aligned}
& \text { Le } 1^{\text {or }} \text { de } 1 \text { an } \\
& \text { Le } 2^{\circ} \text { de } 2 \text { ans } \\
& \text { Le } 3^{\circ} \text { de } 3 \text { ans } \\
& \text { Le } 29^{\circ} \text { de } 29 \text { ans } \\
& \text { Le } 30^{\circ} \text { de } 30 \text { ans }
\end{aligned}
$$

Les 29 premières tiges (parties blanches de la fig. 5) forment le matériel-bois; la $30^{\circ}$ (partie ombrée de la fig. 5) est la coupe annuelle.

(1) Voir Note $B$. 
Le matériel est ainsi de 29 tiges quand on le considère par rapport à la récolte, mais si on examine l'immeuble en entier prêt à livrer sa récolte, cet immeuble doit présenter toujours la suite des 30 tiges et cette suite est alors le matériel dont l'exploitation doit être continuellement garnie.

Dans les exploitations aménagées d'arbres isolés, le matériel-bois est formé par une suite de tiges en nombre égal à l'âge d'exploitation et présentant des âges échelonnés depuis un an jusqu'à l'âge de la coupe. Mais il n’arrive pour ainsi dire jamais que ce matériel soit régulièrement disposé comme la théorie l'indique et comme le montre la figure 5. Les arbres des champs sont eux-mêmes toujours plus ou moins irrégulièrement disposés.

Il est à remarquer que la disposition des tiges formant le matériel-bois n'influe en aucune façon sur la surface occupée par ce matériel. Cette surface, qui est représentée dans la figure 5 par une succession de cercles égaux à la projection de la cime, est toujours constante; elle varie avec l'âge d'exploitation, mais pour un même âge et pour une même essence, la surface occupée est invariable d'étendue, bien qu'il soit nécessaire d'affecter au matériel une contenance plus grande que celle de la surface occupée, puisque les arbres ne sont pas mobiles et ont, en granclissant, un couvert de plus en plus grand. La contenance nécessaire à l'exploitation est au moins égale à un rectangle ayant pour hauteur le diamètre du couvert de l'arbre de coupe et, pour base, autant de fois ce diamètre qu'il y a d'années dans le terme d'exploitation, sauf la déduction due aux interstices des couverts circulaires.

Dans les forêts de jardinage et de furetage, le matérielbois a un arrangement spécial qui est la disposition confuse: toutes les tiges sont mélang'ées entre elles, sans aucun ordre, depuis le brin naissant jusqu'à l'arbre exploitable. La figure 6 indique cette disposition avec la projection des cimes au-dessous du dessin. 
La nature, qui seule fait naître les tiges, ne saurait les disposer dans un ordre quelconque, mais on concoit également que, si on veut obtenir d'un hectare de futaie jardinée, tous les ans un arbre de 150 ans, il faudra qu'il existe sur cet hectare au moins 150 tiges principales de 1 à 150 ans, ou devenant successivement principales par lit sélection naturelle qui s'opère entre toutes les tiges garnissant le sol. Dans cet état de mélange confus, il y a des tiges dominantes et dominées, et si on considère la projection des cimes $(f g .6)$, on voit qu'il y a des recouvrements des unes sur les autres jusqu’à ce que des vides se produisent par l'enlèvement des arbres de coupe ou jusqu'à ce que la vitalité cle l'une l'emportant, celle-ci finisse par devenir dominante.

Cet état explique l'imperfection de ce genre d'exploitation; le sol doit toujours être couvert d'une quantité de tiges en nombre bien plus grand que la suite absolument nécessaire de 150 tiges de 1 à 150 ans. Il est certain que ces 150 tiges principales ne suffisent pas à garnir le terrain et qu'il y a, dans leurs interstices, place pour un remplissage de tiges de même nature. L'exploitant doit considérer que ce remplissage est indispensable à la protection du terrain et que, en outre, il doit tenir compte des déchets et des luttes qui existent pour la vie dans les agglomérations d'arbres, comme dans les sociétés d'êtres vivants.

Nous avons fait remarquer ( $\left.n^{\circ} 64\right)$ que l'exploitation aménagée est formée d'une succession d'exploitations périodiques; il est donc naturel que le matériel-bois de celle-ci se retrouve également dans celle-là et soit compté de même. On pent donc établir, en principe, que dans l'exploitation aménagée, le capital d'exploitation est formé d'un matériel identique à celui des exploitations périodiques, anquel s'ajoute la suite complète de toutes les récoltes pendantes, pour tous les âges, depuis celui du début jusqu’à celui adopté pour l'âge d'exploitation. 
$\mathrm{N}^{\circ} 71$. - Dans les exploitations aménagées de peuplements d'un seul âge, le matériel-bois est formé de la même manière que dans celles d'arbres isolés. C'est, par exemple, une suite de contenances égales âgées la première de 1 an, la deuxième de 2 ans, la troisième de 3 ans, etc., jusqu'à celle de 30 ans. Il est évident que pour obtenir, chaque année et sans interruption, un hectare de 30 ans, il faut posséder 30 hectares, ayant tous les âges échelonnés de 1 à 30 ans ( $\mathrm{fg} .7$ ). Les coupons de 1 à 29 ans forment le matériel-bois, et celui de 30 ans, la récolte ; l'ensemble des 30 coupons de 1 à 30 ans est le capital dont l'exploitation doit être constamment garnie.

Que l'exploitation soit d'arbres isolés ou de peuplements la combinaison est la même et appelle les mêmes remarques :

Le matériel est rarement disposé régulièrement comme dans la figure 7 ; c'est à peine si on rencontre cette régularité dans les taillis et dans les forêts à court terme.

Le matériel est formé à la fois : $1^{\circ}$ par la suite des récoltes pendantes; $2^{\circ}$ par l'ensouchement ou le semis sur toute la surface affectée à l'exploitation.

Au premier abord, il peut paraître singulier de compter les semis sur toute la surface quand il ne se produit que sur le seul coupon en tour de coupe. Rien n'estplas naturel cependant, car le semis se produit successivement sur tous les coupons et doit dès lors être compté comme matériel nécessaire sur toute la surface. Il en est de même de l'ensouchement : il ne paraît que sur la coupe abattue mais il existe réellement d'une manière latente sur toute l'étendue du taillis.

Le matériel d'exploitation aménagée est donc formé : $1^{\circ}$ du matériel de l'exploitation périodique; $2^{\circ}$ de l'ensemble des récoltes pendantes pour tous les âg'es compris entre celui du début et celui de la coupe mûre.

Nous arons remarqué que les exploitations d'arbres iso- 
lés n'occupent sur le sol qu'une suite d'espaces circulaires décroissants représentés par la projection des cimes avec les interstices nécessaires. Lorsque ces arbres sont réunis par contenance comme dans le jardinage, ces interstices sont occupés par un remplissage non utilisé et servant à la garniture du sol. Dans l'exploitation de peuplements, ceux-ci sont enfermés sur des surfaces limitées qui se joignent sans lacune; on compte le travail des peuplements, non plus par arbre, mais pour leur ensemble, et les exploitations de peuplements semblent utiliser tout le sol sans aucun déchet. Mais dans l'intérieur du peuplement les interstices des cimes circulaires des arbres n'en existent pas moins et laissent passer une lumière diffuse à la faveur de laquelle naît un sous-étage couvrant également le sol et également utile à sa conservation.

La production générale est-elle la même? Il y a, de ce côté, une question encore indéterminée et dont la solution dépend de l'écartement des tiges qui forment le peuplement. En tout cas, l'utilisation du sol est différente; l'exploitant consacre une surface à chaque âge ; il peut ainsi réaliser plus de produits que dans les forêts jardinées, car le mélange confus de celles-ci l'oblige à faire une part, assez large aux exigences de la sélection naturelle, toujours lente et indéterminée, et à modérer sa jouissance en raison de l'ignorance de la manière dont les âges sont groupés, à moins que l'activité de croissance des arbres isolés (certainement plus grande que celle des arbres réunis en peuplements) ne compense cette imperfection et ne produise une réalisation aussi grande dans un cas que dans l'autre pour un même âge d'exploitation. Le doute qui existe sur cette question dans l'état actuel de la science nous a fait conseiller $\left(\mathrm{n}^{\circ}\right.$ 69) les exploitations mixtes d'arbres et de peuplements, parce qu'elles réunissent les avantages de la fabrication du bois par l'arbre isolé et par le peuplement. 
$N^{\circ}$ 72. - Le matériel-bois des exploitations aménagées mixtes d'arbres et de peuplements est plus compliqué. Prenons pour exemple 50 hectares de pins aménagés à cinquante ans à raison de 1 hectare par an, sur lequel on réserve 40 pins de choix. Le revenu est formé: $l^{\circ}$ par un hectare du peuplement de pins (moins les 40 arbres), et: $2^{\circ}$ par 40 modernes de 100 ans.

Le matériel d'exploitation se compose d'abord de la suite de ces 50 hectares échelonnés de 1 à 50 ans, et ensuite de 50 groupes de 40 pins isolés, âgés de 50 à 100 ans et disposés comme le montre la figure 8 , dans laquelle il n'a pas été possible de dessiner les 40 arbres de chaque groupe. Ils ont été figurés par un trait noir comme s'il n'y avail qu'un arbre de revenu chaque année; ce trait de plus en plus épaissi à mesure qu'on avance vers la coupe mûre exprime le grossissement successif de ces tiges.

Dans le matériel de l'exploitation en arbres qui doit fournir un arbre de 100 ans en revenu, chaque année (en réalité 40 arbres par an), la suite des 100 arbres de 1 à 100 ans existe bien au complet, mais comme il n'y a pas de terrain pour l'installer en dehors des 50 hectares, cette suite a été repliée sur elle-même comme l'indique la figure 8 . Ce repliement en 2 tronçons a pour résultat que la partie de 1 à 50 ans est en quelque sorte noyée dans le peuplement; les tiges n'y sont pas encore isolées par la main de l'homme. Le tronçon de 50 à 100 ans est seul formé de tiges dégagées et mises en réserve par une épargne de 50 ans en sus de celle qui a formé originairement le peuplement.

C'est pour ce motif que le balivagé seul est compté comme matériel d'exploitation, quand on considère celleci comme prête à fonctionner et à fournir des arbres de 100 ans.

La même remarque a été faite pour les exploitations 
périodiques de même nature ( $\mathrm{n}^{\circ}$ 69, $\mathrm{fg}$. 3). Elle montre que, pour évaluer le matériel de l'exploitation aménagée, on peut procéder de deux manières : soit en évaluant ce que vaut la suite d'arbres de 1 à 50 ans, mais en ne comptant le peuplement que distraction faite des tiges de 1 à 50 ans; - soit en évaluant les arbres de 50 à 100 ans, mais en estimant alors les peuplements au complet. Cette seconde méthode est la plus pratique et la plus suivie.

Le repliement de la suite d'arbres de là 100 ans en deux tronçons permet de représenter encore l'exploitation par un diagramme ( $f g .9)$ dans lequel la ligne supérieure ascendante de 50 à 100 ans indique les arbres du balivage, et dont la largeur est proportionnelle au nombre d'arbres. Le triangle inférieur avec sa ligne ascendante de 0 à 50 ans exprime le peuplement.

On peut aussi représenter le nombre d'arbres par un rectangle construit à la base de chaque coupe; la largeur de ce rectangle exprime le nombre, et la hauteur, l'âge des arbres ; les parties blanches du diagramme ( $f g .10)$ montrent les parties de récoltes pendantes qui forment le matériel-bois de l'exploitation en arbres.

De sorte que ce diagramme indique que dans celte exploitation mixte, le matériel-bois est composé, comme dans les précédentes, des récoltes pendantes et du matériel initial. En d'autres termes, le matériel-bois de l'exploitation aménagée comprend le matériel de l'exploitation périodique, anquel s'ajoute l'ensemble des récoltes pendantes du peuplement et des arbres.

La même constitution s'applique aux taillis sous futaie simple, c'est-à-dire à ceux dont la futaie ne doit acquérir qu'un âge en sus de celui du taillis et est ainsi coupée toute à l'état de moderne pour être remplacée par des baliveaux en nombre égal, sauf le déchet de ceux-ci dont il faut toujours tenir compte dans la pratique. 
$N^{\circ}$ 73. - Exploitations graduées. - Entre l'exploitation aménagée qui fournit chaque année du bois de 30 ans par exemple et l'exploitation périodique qui ne procure ce bois que chaque 30 ans, il existe une exploitation intermédiaire qui n'a pas encore reçu de nom spécial, mais qu'il importe de signaler à cause de son utilité.

Elle consiste à fournir des bois de l'âge adopté, non pas chaque année, mais à des intervalles réguliers et égaux entre eux, supérieurs à une année et inférieurs à l'âge d'exploitation, par exemple des bois de 30 ans tous les 2 ans ou tous les 3 ans; elle convient ainsi aux forêts de petite étendue.

La figure 11 montre la disposition de cette exploitation pour 30 hectares de taillis exploités en 6 coupons de 5 hectares à raison de 1 tous les 5 ans. La fugure 12 indique une exploitation de la même contenance réglée en 2 coupons de 15 hectares à raison de 1 coupon tous les 15 ans.

L'examen de ces deux figures montre que si on compare cette exploitation à celle aménagée, et si on considère la forêt au moment où la coupe mûre va être abattue, l'immeuble présente un état boisé plus considérable et de valeur plus grande que dans l'exploitation aménagée. Si on fait cet examen après la récolte de la coupe mûre, cet état boisé est, au contraire, moins considérable. L'état de l'immeuble est donc intermédiaire entre celui de l'exploitation aménagée et celui de l'exploitation périodique.

Mais le matériel d'exploitation considéré par rapport à la récolte, c'est-à-dire la quantité de bois qu'il est nécessaire de maintenir sur pied pour obtenir la récolte, est toujours moindre que le matériel de l'exploitation aménagée. Ainsi dans la figure 11, les 5 coupons $n^{05} 1$ à 5 , formant 25 hectares, ont moins de valeur que les coupons $\mathrm{n}^{\circ 5} 1$ à 29 , formant 29 hectares de l'exploitation aménagée. 
Or, dans toute entreprise, la diminution du capital a pour effet d'amoindrir le produit. Cet effet économique se manifeste dans l'exploitation graduée :

Si l'hectare de 30 ans vaut 1,000 francs, le propriétaire d'une exploitation graduée d̀ 5 ans touchera 5,000 francs chaque 5 ans, et celui de la forêt aménagée 1,000 francs tous les ans. Or, six annuités périodiques de 5,000 francs rendent moins de services et ont moins de valeur que 30 annuités de 1,000 francs recueillies dans le même laps de temps de 30 ans. Il suffit, pour s'en convaincre, de placer les unes et les autres à un taux quelconque, $50 / 0$ par exemple, et de comparer les sommes produites:

30 annuités de $1000 \mathrm{fr}$. forment au bout de 30 ans $1000 \times 3,3219 \times 20=66438$.

6 annuités périodiques de 5000 valent $5000 \times 3,3219 \times 3,619=60110 \mathrm{fr}$.

( $\left.\mathrm{N}^{\circ} 44.\right)$

On voit, en outre, que le matériel initial de l'exploitation périodique (ensouchement) existe au complet et de la mème manière, dans cette exploitation comme dans l'exploitation aménagée, mais les récoltes pendantes, qui sont la caractéristique de celle-ci, y sont toujour's moins importantes; elles diminuent à mesure que l'intervalle des coupes augmente et tendent, selon le cas, vers le minimum, qui est le caractère propre de l'exploitation périodique : pour celle-ci la récolte pendante est nulle dans le matériel d'exploitation.

D'un autre côté, l'état de la superficie de la forêt considérée au moment de la maturité de la coupe, augmente à mesure que l'intervalle des coupes grandit, pour se rapprocher de l'exploitation périodique, où cette superficie est à son maximum quand la coupe est mûre.

$N^{\circ}$ 74. - Les exploitations graduées sont très utiles dans la gestion des petites fortunes, quand le propriétaire ne possècle pas une contenance suffisante pour que l'échelle complète des âges lui fournisse une coupe facile à vendre.

On possede 15 hectares de taillis; aménagés à 15 ans, ils 
produiraient une coupe annuelle d'un hectare; mais le propriétaire estime avec raison que des bois de 30 ans se vendent à des prix plus élevés; s'il règle son exploitation à cet âge, il n'obtiendra qu'un demi-hectare de coupe annuelle, contenance trop faible pour attirer les marchands de bois; il aura tout avantage à établir une exploitation graduée à 2 ans qui lui fournira 15 coupes de 1 hectare à réaliser chaque 2 ans.

On voit que les combinaisons d'exploitation s'adaptent ainsi à tous les besoins et à toutes les fortunes.

L'exploitation graduée peut se pratiquer pour les forêts de nos trois types, c'est-à-dire pour les forêts d'arbres isolés, de peuplements d'un seul âge et pour les forêts mixtes d'arbres et de peuplements. Il suffit de remarquer que du moment où la récolte peut se percevoir chaque année, elle peut tout aussi bien se réaliser tous les 2 ans, tous les 3 ans, etc.

$N^{\circ}$ 75. - Un autre avantage des exploitations graduées est de servir à monter des exploitations aménagées dont elles forment les éléments, de la même manière que nous avons vu l'exploitation périodique servir d'élément à l'exploitation aménagée.

C'est aux forêts qui sont aménagées par leur nature (jardinage et furetage) que cette observation s'applique. Ces forêts seraient vite fatiguées si on y prenait chaque année le revenu annuel. En outré, lorsque ces forêts sont d'une grande étendue, il devient fort difficile d'en recueillir le revenu qu'il faut glaner sur chaque hectare. Dès lors, on peut établir une exploitation aménagée qui sera formée d'exploitations graduées en nombre égal aux intervalles de celles-ci. En prenant la coupe sur chacune et en suivant de proche en proche et toujours dans le même ordre, on possédera une exploitation aménagée à produits annuels constants.

Voici, par exemple, 10 hectares de forêt jardinée fournissant naturellement 10 sapins par an, d'une dimension corres- 
pondant à 140 ans et qui, depuis longtemps, se trouvent réglés de manière à fournir 100 sapins de cette dirnension tous les 10 ans.

C'est une exploitation graduée dans laquelle la forêt présente en tiges principales, et, en outre du remplissage, 1,400 tiges graduées.

$$
\begin{array}{r}
100 \text { arbres de } 140 \text { ans } \\
100-\text { de } 130 \text { ans } \\
100-\text { de } 120 \text { ans } \\
\ldots \ldots \ldots \ldots \ldots \ldots \\
100 \text { arbres de } 20 \text { ans } \\
\frac{100}{1400} \text { - de } 10 \text { ans }
\end{array}
$$

On remarque qu'il y a ici le même nombre de tiges principales que dans une exploitation aménagée, mais ces tiges sont de plus grande valeur, puisqu'elles existent par dizaines de $140,130,120 \ldots$ ans, sans les âges intermédiaires qui existent dans la suite de 1,400 tiges, de 1 à 140 ans. C'est le même état que nous avons remarqué dans l'exploitation de peuplements de la figure 11.

Or, si la forêt présente 100 hectares, formés de 10 parcelles composées rle semblable manière, on pourra substituer à la coupe décennale de 1,000 sapins, une coupe annuelle de 100 arbres demandés à chaque coupon à tour de rôle et en ne revenant sur le même que tous les 10 ans.

Par l'effet de cette rotation, les 10 coupons présenteront en tiges de sélection :

Le premier.

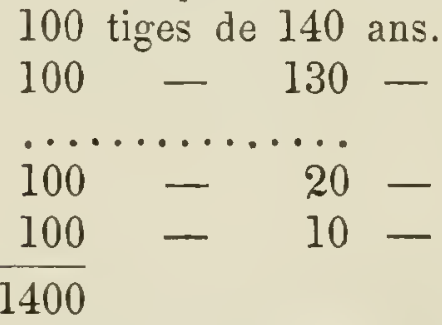

Le troisième.

100 tiges de 138 ans.

$100-128-$

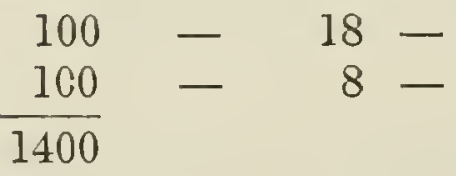

Le second.

100 tiges de 139 ans.
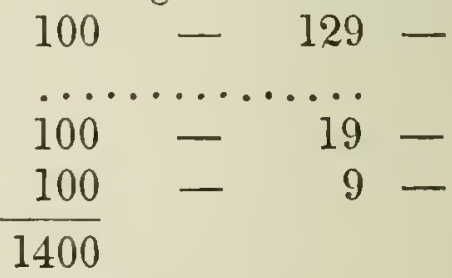

Le dixième.

100 tiges de 131 ans.

$100-121-$

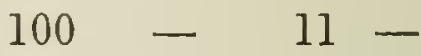

On roit que, par l'effet de cette combinaison, on obtient les 
10 suites de 140 tiges échelonnées de 1 à 140 ans et repliées 10 fois sur elles-mêmes pour être disposées sur les 10 coupons de la forêt. La suite des âges existe sans aucune lacune pour' constituer le matériel de l'exploitation aménagée.

Ce n'est pas la théorie qui a conduit à cette combinaison, c'est au contraire la pratique qui l'a inspirée par une longue tradition. La forêt s'est trouvée trop grande pour qu'on puisse recueillir sur chaque hectare le sapin mûr que son état, naturellement aménagé, lui permettait de livrer. On a été naturellement conduit à prendre ces 100 arbres sur un canton limité; l'année suivante, on a fait de même en marchant de proche en proche, canton par canton et en parcourant la forêt en 10 ans. Avec le temps, ces cantons, plus faciles à vendre et à surveiller, se sont localisés et sont devenus égaux, chacun a formé une exploitation graduée; l'ensemble a créé un aménagement où les âges se suivent sans interruption, en supposant toutefois que la nature ait créé les tiges avec la même régularité, circonstance dont il n'y a pas lieu de s'inquiéter à cause du remplissage caractéristique de ces forêts.

Le furetage comporte le même état d'exploitation naturellement aménagée. Il est difficile de couper chaque année sur toute l'étendue d'une forêt les lances ou perches de mesure adoptée; c'est à peine si un propriétaire soigneux peut agir ainsi pour un bois de petite étendue. Dans une grande forêt, la récolte annuelle serait trop délicate et le massif n'aurait pas le temps de repos nécessaire.

Gn peut adopter un intervalle de neuf ans pour revenir 3 fois sur le même coupon si la lance se produit en 27 ans. Une forèt de 90 hectares pourra ètre divisée en 9 coupons de 10 hectares, qui présenteront la succession d'âges suivante:

$\begin{array}{lrrrrrrrrr}\text { Coupons nos } & 1 & 2 & 3 & 4 & 5 & 6 & 7 & 8 & 9 \\ \text { Tiges de } & 27 & 26 & 25 & 24 & 23 & 22 & 21 & 20 & 19 \text { ans } \\ & 18 & 17 & 16 & 15 & 14 & 13 & 12 & 11 & 10- \\ & 9 & 8 & 7 & 6 & 5 & 4 & 3 & 2 & 1-\end{array}$

La première année, on coupera dans le coupon $n^{\circ} 1$ les tiges de 27 ans ayant acquis, par exemple, $0^{\mathrm{m}}, 10$ de diamètre; la 
seconde année, on passera au coupon $n^{0} 2$ dont les tiges auront 27 ans, et ainsi de suite; après 9 ans, on reviendra sur le coupon $\mathrm{n}^{\circ} \mathrm{l}$ pour $\mathrm{y}$ trouver des tiges de 27 ans et en continuant ainsi, on aura renouvelé entièrement le matériel d'exploitation en 3 rotations de 9 ans, c'est-à-dire en 27 ans.

Cette formation des exploitations aménagées au moyen de parcelles à exploitation graduée, ne s’applique qu'aux forêts dont les âges sont confusément mêlés, et qui sont ainsi naturellement aménagées. Les groupements aménagés formés par d'autres traitements culturaux, par exemple avec des taillis sous futaie, n'ont que l'apparence de cette combinaison, parce que la parcelle ne contient pas la suite complète des âges, tandis qu'ici chaque coupon contient des tiges équivalentes, sinon en âge, du moins en nombre, à la suite aménagée complète.

$\mathrm{N}^{\circ}$ 76. - Les explications précédentes montrent que pour des forêts dont l'unité de contenance est naturellement aménagée, telles que le jardinage et le furetage, on peut constituer, sur de grandes surfaces, des exploitations aménagées avec des éléments gradués. Il n'est pas besoin de beaucoup d'imagination pour comprendre qu'on peut également constituer des exploitations graduées quand on ne possède que de moyennes surfaces.

Il suffit dans l'exemple précédent, de supposer que au lieu de cent hectares divisés en 10 coupons de 10 hectares, on ne possède que 50 hectares divisés en 5 coupons de 10 hectares. L'exploitant pourra demander à chacun la double production tous les deux ans et constituer ainsi l'exploitation graduée à raison d'une coupe de 10 hectares chaque deux ans.

Il en est de mème pour les 90 hectares de furetage exploités en 9 coupes annuelles de 10 hectares. Une forêt de 30 hectares pourrait être exploitée en 3 coupes de 10 hectares tous les 3 ans.

Les forêts à peuplements d’âges confusément mélangés formant des exploitations aménagées sur la plus petite 
contenance sont ainsi propres aux besoins des plus petites fortunes; elles se prêtent, aussi, par la combinaison d'éléments gradués que nous venons d'expliquer, à la gestion des grands domaines $\left(n^{\circ} 75\right)$ et des propriétés moyennes. Il en résulte que les forêts de cette nature sont toujours divisibles et partageables sans nuire à la constitution de l'exploitation. C'est un avantage considérable sur les forêts de peuplements d'un seul âge. Si on partage, par exemple, un taillis aménagé à 30 ans entre deux héritiers, le matériel divisé se prêtera difficilement pour chacun à une exploitation de même nature. L'un, obtenant les bois âgés, sera disposé à les couper; l'autre, n'ayant que des bois jeunes, sera forcé d'attendre leur maturité. La suciété pourra souffrir plus ou moins de la division de ces exploitations entre héritiers; elle n'en souffre jamais quand il s'agit de forêts jardinées ou furetées.

\section{§3. - Exploitations composées.}

Nos 77. - Exploitations périodiques d'arbres isolés.
78. - - - - de peuplements d'un seul âge.
79. - - - - mixtes d'arbres et de peuplements.
80. - Remarque sur l'effet de l'augmentation du matériel-bois.
81. - Exploitations aménagées : arbres isolés.
82. - - - - forêts de peuplements d'un seul âge.
83. - - - forèts mixtes d'arbres et de peuple- ments.
84. - Exploitations graduées : combinaisons diverses.

\section{$\mathrm{N}^{\circ}$ 77. - Exploitations périodiques. - La futaie} étagée dont nous avons donné la description $\left(\mathrm{n}^{\circ} 58\right)$ forme une exploitation périodique d'arbres isolés par la main. de l'homme et dont le caractère est d'être composée, c'est-àdire de fournir, à chaque période, des arbres de diverses dimensions.

On constate, par exemple, que sur un hectare il existe 390 
tiges mélangées de hêtres et de sapins couvrant le sol sans lacune et présentant l'état suivant:

\begin{tabular}{|c|c|c|c|}
\hline \multicolumn{4}{|c|}{120 tiges } \\
\hline 110 & - & 0,20 & - \\
\hline 88 & - & 0,30 & - \\
\hline 52 & - & 0,40 & - \\
\hline 17 & - & 0,50 & - \\
\hline 3 & - & 0,60 & - \\
\hline
\end{tabular}

Pour régler la coupe, on admet, par exemple, qu'il faut en moyenne 28 ans pour faire gagner à un arbre 10 centimètres en diamètre et que si on coupe les différences qui existent entre les nombres de chaque catégorie, le massif se reconstituera en 28 ans tel qu'il existait auparavant. Le sousétage présentera, au moment de la coupe, un semis incomplet mais capable de fournir de jeunes brins en nombre égal aux arbres abattus et capables de les remplacer un jour :

\begin{tabular}{|c|c|c|c|c|c|c|}
\hline 120 & - & 110 & $=$ & 10 & res & e $0,{ }^{\mathrm{m}} 10$ \\
\hline 110 & - & 88 & $=$ & 22 & - & 0,20 \\
\hline 88 & - & 52 & $=$ & 36 & - & 0,30 \\
\hline 52 & - & 17 & $=$ & 35 & - & 0,40 \\
\hline 17 & - & 3 & $=$ & 14 & - & 0,50 \\
\hline 3 & - & 0 & $=$ & 3 & - & 0,30 \\
\hline
\end{tabular}

on a: 390 ; on laisse 270 ; on coupe 120 arbres on ajoute 120 brins

on maintient 390 arbres

En nettoyant l'aire de la coupe de tout le sous-étage, sauf ces 120 brins, c'est-à-dire en exploitant l'hectare de coupe à tire-aire et en réservant ces 390 tiges, on obtiendra un semis suffisant pour protéger le terrain et reproduire le même état à la coupe suivante.

Naturellement, dans la pratique, les choses ne se présentent pas avec cette régularité absolue. Il ne s'agit ici que de la conception théorique de l'exploitation, c'est-àdire d'une règle que les faits ne suivent jamais entièrement et qui est simplement destinée à éclairer l'exploitant sur la manière dont il s'en écarte ou s'en approche.

Une exploitation périodique de cette nature a pour ma- 
tériel-bois, outre le semis naturel sur toute la surface, un balivage de 390 tiges ainsi composé par hectare:

$\begin{array}{rrrr}120 & \text { brins } & \prime \prime & \prime \prime \\ 110 & \text { arbres de } & 0^{\mathrm{mc}}, 10 \\ 88 & - & 0 & , 20 \\ 52 & - & 0 & , 30 \\ 17 & - & 0 & , 40 \\ 3 & - & 0 & , 50\end{array}$

Ces futaies étagées étant d'une constitution analogue à celle des futaies composées sur taillis, nous renvoyons au $n^{\circ} 79$ pour les observations relatives à leur constitution. Elles n'en diffèrent, en effet, qu'en ce que le taillis est ici entièrement sacrifié; les arbres sont en nombre aussi grand que possible et la forêt n'est qu'une exploitation d'arbres. Il en résulte que dans la pratique et quand la forêt est bien constituée en arbres, c'est-à-dire, bien pleine d'arbres de différents âges, on se contente de couper les tiges trop serrées et hors d'état de prospérer jusqu'à la coupe suivante. C'est la formule pratique des anciennes ordonnances.

$\mathrm{N}^{\circ}$ 78. - Une exploitation de peuplements d'un seul âge peut être simple ou composée; elle est simple quand elle est réduite au peuplement de l'âge fixé (50 ans, par exemple, pour une pineraie aménagée à 50 ans) [voir $n^{\circ} 68$; elle est composée quand, en sus de ce peuplement principal, on recueille chaque année des produits intermédiaires provenant des éclaircies faites, par exemple, à 20, 30 et 40 ans.

Ces produits des éclaircies sont dus à des exploitations intermédiaires qui n’ont pas de matériel-bois spécial, comme nous le verrons également pour le jardinage composé $\left(n^{\circ} 81\right)$.

Voici, en effet, sur quelle observation est fondée la pratique des éclaircies. Si on considère un hectare de pins 
qui viennent de naître et si on l'examine à ses différents âges jusqu'à 50 ans, on constate que le nombre des tiges a successivement diminué par l'effet de la lutte pour la vie. Le nombre des tiges qui était, par exemple, de 10,000 à 1 an, n'est plus que de 600 à 50 ans, sans qu'on en ait coupé aucune et par le seul effet de la mortalité naturelle. Le peuplement de 50 hectares présente par exemple:

à 1 an 10000 tiges par hectare

à 20 ans 3000 -

à 30 ans 1400 -

à 40 ans 900 -

à 50 ans 600 -

$\mathrm{Au}$ lieu de laisser les tiges débiles se détruire successivement par l'action naturelle, on a imaginé de les recueillir par anticipation au moyen de coupes qui se font quand les peuplements ont acquis 20, 30 et 40 ans : sur l'hectare de 40 ans, par exemple, qui présente 900 tiges, on en enlève 300 environ, de manière à le mettre au nombre où il sera à 50 ans et ainsi de suite:

sur l'hectare de 40 ans ayant 900 tiges, on coupe 300 tiges,

$$
\text { - de } 30 \mathrm{ans}-1400 \text { - } 20 \text { - } \begin{array}{r}
500 \text { ans } \\
\text { - }
\end{array}
$$

Quant aux 7,000 tiges qui disparaissent de 1 à 20 ans, on les considère comme non utilisables et devant disparaître par l'action naturelle, de sorte que les quantités coupées ou vouées à cette destruction naturelle représentent bien les 10,000 tiges du début.

$$
\begin{array}{rrrr}
\text { Coupe à } 50 \text { ans } & 600 \text { tiges } \\
- & 40 \text { ans } & 300 & \text { - } \\
- & 30 \text { ans } & 500 & \text { - } \\
- & 20 \text { ans } & 1600 & \text { - } \\
\text { Mortalité de } 1 \text { à } 20 \text { ans } & 7000 & \text { - } \\
& \text { Total } &
\end{array}
$$

Naturellement ces chiffres ne sont qu'une indication du 
mécanisme de l'exploitation. On ne connaît pas encore la loi de décroissance du nombre des tiges avec l'âge du peuplement et l'éclaircie est une pratique entièrement culturale sur laquelle les sylviculteurs ne sont pas encore tous d'accord $\left({ }^{1}\right)$.

On voit, en tout cas, que le peuplement existerait avec sa décroissance naturelle dans le nombre des tiges et que l'éclaircie ne fait que hâter l'action de la nature en utilisant des bois qui se perdraient sans elle. Cette exploitation intermédiaire n'a donc pas de matériel spécial ; il suffit d'évaluer les peuplements de chaque âge au rolume et à la valeur qu'ils ont à cet âge, pour comprendre le matériel qui suffit à assurer perpétuellement le produit principal et les produits intermédiaires.

$\mathrm{N}^{\circ} 79$. - Un hectare de taillis sous futaie peut former une exploitation composée à double titre. Le taillis qui est un peuplement d'un seul âge peut fournir une éclaircie destinée à favoriser des essences précieuses et la futaie peut livrer des arbres de diver's âges, des modernes, des anciens et des bis-anciens.

La constitution de l'exploitation de ces arbres isolés sur le peuplement est, dans ce cas, beaucoup plus compliquée que celle de la futaie à un seul âge, élevée sur le taillis dont les forêts ont été étudiées au n 72, sous le nom de taillis sous futaie simple. Dans le cas qui nous occupe, la futaie est composée d'arbres de plusieurs âges

( $\left.{ }^{1}\right)$ Dans un peuplement formé de iiges nées à la même époque, on remarque des liges de trois états différents et dites dominantes, dominces et retardataires. Pendant longtemps on n'a pratiqué l'éclaircie que sur les tiges dominées; depuis un certain nombre d'années, on laisse ces tiges dominées en protection du sol et on fait porter l'éclaircie sur les liges retardataires pour favoriser les dominantes. Et voilà (jue II. Borggreve, directeur de l'école lorestière de Munden, propose de laire porter la coupe d'éclaircie sur les tiges domiuntes, de façon a réaliser de meilleurs produits et á faroriser la végítation des retardataires (Die IIol:zucht, 1 rol, in-80, Berlin, 1885). 
et la forèt prend naturellement le nom de taillis sous futaie composée.

Un hectare de taillis sous futaie s'exploite périodiquement à 30 ans, avec la condition de réserver sur chaque coupe 110 arbres : 60 baliveaux de 30 ans, 40 modernes de 60 et 10 anciens de 90 ans.

La réserve ainsi détaillée est ce qu'on appelle le plan de balivage.

Dans 30 ans, la coupe présentera :

110 arbres $\left\{\begin{array}{l}60 \text { modernes; } \\ 40 \text { anciens; } \\ 10 \text { bis-anciens. }\end{array}\right.$

Le revenu périodique sera fourni par les diffẻrences entre ces classes d'âges.

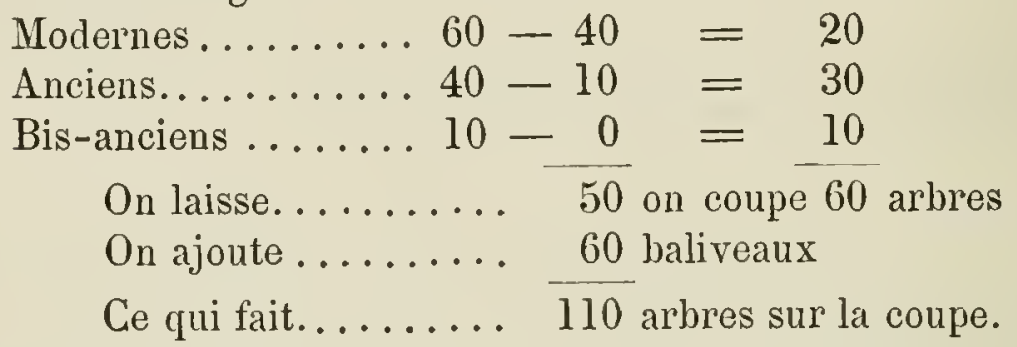

On voit qu'en coupant tous les 30 ans 20 modernes, 30 anciens et 10 bis-anciens (60 arbres) et en laissant sur pied 60 baliveaux réunis aux 50 arbres non coupés, on aura un revenu périodique constant de 60 arbres et une forêt outillée perpétuellement du même plan de balivage.

Le matériel-bois peut être représenté par la figure 13 et la coupe par les parties blanches de la figure 14. Le matériel et la coupe peuvent également se représenter par les figures 15 et 16 dans lesquelles les rectangles noirs figurent le matériel d'exploitation et les parties blanches la récolte à réaliser.

On concoit l'origine de ces taillis sous futaie composée; le propriétaire a débuté par un taillis sous futaie simple dont le matériel était formé par la modeste économie d'un certain nombre cle baliveaux de 30 ans. Lors de la coupe, le propriétaire voyant ses arbres bien venants et voulant en produire de plus gros et de plus utiles, n'a pas abattu tous les modernes. Il en a choisi un certain nombre par- 
mi les meilleurs pour parcourir une nouvelle période de 30 ans: sur ses 60 modernes, il n'en a abattu que 20 (les plus mauvais) et en a gardé 40 (les meilleurs). A la coupe suivante, il possédait une exploitation montée à 20 modernes et 40 anciens de revenu, mais il a continué son épargne et, toujours dans le but d'améliorer son exploitation, il n'a coupé que trente anciens, en laissant 10 pour la prochaine coupe.

L'exploitation a été dès lors montée sur un revenu de:

$$
\begin{aligned}
& 20 \text { arbres de } 60 \text { ans } \\
& 30 \text { - de } 90 \text { ans } \\
& 10-\text { de } 120 \text { ans }
\end{aligned}
$$

se reproduisant indéfiniment tous les 30 ans; mais il a fallu pour arriver à ce résultat une économie de plus fortes sommes et soutenue pendant 90 années.

Dans les exploitations d'arbres et de peuplements le matériel d'exploitation est donc formé : $1^{\circ}$ du semis ou de l'ensouchement; $2^{\circ}$ du plan de balivage.

$\mathrm{N}^{\circ}$ 80. - L’observation faite au numéro précédent sur la manière dont les exploitations à arbres de réserves ont été constituées par l'épargne, appelle une remarque économique importante.

L'économie politique montre que dans toute entreprise l'augmentation du capital a pour effet d'améliorer les produits de l'exploitation.

Le même phénomène se manifeste en économie forestière: l'augmentation du matériel-bois n'a pas pour effet d'augmenter la quantité des produits; car l'action des agents naturels, étant toujours à peu près identique, ne modifie pas sensiblement le poids et le volume de matière ligneuse fabriquée; mais elle a pour effet d'accroître la qualité des marchandises produites dans le même temps.

Avec un matériel réduit à l'ensouchement, l'exploitation on 
taillis ordinaire ne peut produire, tous les 30 ans, que des tiges de 30 ans; qu'on ajoute au matériel de l'ensouchemen quelques baliveaux de 30 ans, c'est-à-dire le fruit d'une épargne de 30 ans, l'exploitation ainsi dotée fournira, en outre des taillis, des bois de 60 ans tous les 50 ans. Constituez le matériel-bois avec des arbres de 30 , de 60 et de 90 ans, et vous obtiendrez une exploitation à bois capable de produire des arbres de 60 , de 90 et de 120 ans, régulièrement tous les 50 ans. Il est incontestable que plus un arbre vieillit et grossit, plus il rend de services et plus il a d'utilité et de valeur.

Considérez 1 hectare de futaie pleine de 120 ans qui forme une exploitation périodique à 120 ans; celle-ci fournira ces précieux arbres de 120 ans tout aussi bien que le taillis sous futaie composé, mais elle ne les livrera à la consommation que tous les 120 ans, parce qu'elle n'a qu'un faible matériel-bois (le semis initial). L'exploitation de la futaie sur taillis au contraire, livre ces mêmes arbres tous les 30 ans, c'est-à-dire à des intervalles bien plus rapprochés et d'une manière autrement utile au producteur et au consommateur.

Cette exploitation en futaie pleine produira également des bois de 30 , de 60 et de 90 ans, résultant des éclaircies, mais elle ne les produira que tous les 120 ans, c'est-à-dire qu'après avoir recueilli une première fois des bois de 30,60 et 90 ans, le propriétaire devia attendre 120 ans pour qu'ils reviennent. Là est un autre effet de la constitution de cette exploitation à faible capital : non seulement elle recule la jouissance des produits les plus précieux, mais, en outre, elle r'etarde également celle des plus ordinaires, parce qu'il faut attendre la perception des plus précieux.

Voilà donc un effet très remarquable de l'augmentation du capital-bois; elle accroît la qualité des produits et permet de les obtenir en un temps moindre que leur âge. Dans l'exemple précédent, on obtient des arbres de 120 ans tous les 30 ans par une épargne de produits continuée pendant 90 ans. Or, si on vient à détruire ce matériel d'exploitation, il faudra recommencer à nouveau cette longue épargne; on ne pourra la reconstituer qu'en. 90 ans ; l'outillage faisant défaut, la quantité de la production pourra subsister, mais la qualité manquera ainsi que le prix. 
Aussi les anciennes ordonnances forestières, celle de 1669 notamment, due au prévoyant génie de Colbert, avaient la sagesse de protéger les arbres des taillis contre toutes les tentations; il fallait un rapport spécial des agents de la maîtrise et une ordonnance royale pour permettre d'en couper, tant on avait peur de compromettre une épargne si précieuse.

De nos jours, les Agents forestiers sont libres de couper les arbres des taillis comme ils l'entendent; c'est une haute confiance que les pouvoirs publics ont mise en eux; mais c'est peut-être un danger pour la fortune publique que de ne point les prémunir assez contre le désir qu'ils peuvent éprouver d'augmenter les produits du présent aux dépens des ressources de l'avenir. Qui sait, si les forêts de taillis sous futaie n'étaient pas mieux outillées en arbres sous le régime de l'édit de 1669, que sous celui de l'ordonnance de $1827\left({ }^{1}\right)$ ?

No 81. - Exploitations aménagées. - Dans les forêts jardinées où les arbres isolés par l'action de la nature sont toujours accompagnés d'un remplissage considérable, lorsque l'exploitant n'assigne à ce remplissage que le rôle de protéger le terrain et lorsqu'il limite son revenu à l'arbre de la dimension qu'il s'est fixée, le jardinage est simple. Il est composé, au contraire, lorsque l'exploitant, tout en laissant à ce remplissage son rôle protecteur, lui demande, en outre du revenu principal (un arbre de $150 \mathrm{ans}$ ) quelques arbres d'âges inférieurs, par exemple un arbre de 80 ans et deux de 50 ans. Dans cette exploitation, la forêt fournit alors, chaque année, un sapin de

(1) La Statistique forestière en 1878 constate que les 156,000 hectares de taillis sous futaie domaniaux susceptibles de produire du chêne ont 31 arbres (38 baliveaux, 10 modernes, 3 anciens) par hectare, balivés en 1876 . Les 906,000 hectares en forèts communales de mème nature présentent 86 arbres par hectare (63 baliveaux, 18 modernes, $气$ anciens). 
sciage, un de charpente, deux perches $\left({ }^{1}\right)$. Or, que le remplissage de tiges secondaires soit ainsi utilisé ou qu'il soit considéré uniquement comme la garniture nécessaire du sol, il n'en existe pas moins au complet et doit toujours étre compté dans le matériel d'exploitation : car le traitement en jardinage ne saurait s'en passer et le sylriculteur doit appliquer ses soins à le maintenir toujours en état conrenable et complet.

Un hectare de forét capable de fournir chaque année un arbre de 150 ans, doit contenir forcément 150 tiges âgées de 1 à 150 ans. Ces 150 tiges ne sauraient occuper par leur courert toute la surface de l'hectare, mais la nature ne saurait produire ces 150 tiges arec la régularité absolue de la conception théorique; elle crée les jeunes semis en grand nombre, les perches, les arbres moyens et les grands arbres en nombre de plus en plus restreint suivant une décroissance dont la loi n'est pas encore connue. Cet ensemble accessoire de tiges plus ou moins ágées occupe le restant de la surface.

Dans ces tiges où s'établit la lutte pour la vie et où se forment les remplacants des arbres de coupe, il y a place pour des enlèrements de tiges intermédiaires exposées à périr, comme nous l'avons vu pour les éclaircies des peuplements d'un seul âge. Mais on conçoit combien ces produits doirent étre perçus arec modération, car il est impossible de se rendre compte de la constitution exacte de l'état jardiné et on ne sait jamais si la tige dominée ne deviendra pas un jour un arbre de remplacement. Dans les foréts de peuplements, au contraire, chaque áge ayant, en quelque sorte, une contenance qui lui est affectée, on peut se rendre compte de son état et enlever

(1) M. Gurot, professeur de droit à l'école forestière, a montré des exemples de ce jardinage composé dans les Foréts lorraines, Nancy, un rol. in-80, $188^{\circ}$. 
les tiges surabondantes avec une certaine prévision de l'avenir. On a dit avec raison que la modération est la règle du jardinage; cette règle est surtout celle du jardinage composé.

Le matériel des exploitations en jardinage est formé, à la fois, de la suite des tiges principales et de toutes celles de remplissage que la nature produit suivant une loi encore indéterminée.

Il est à remarquer, en outre, que dans les exploitations aménagées, le matériel-bois est composé de deux parties :

Les récoltes en croissance;

Le matériel de l'exploitation périodique.

Les récoltes en croissance sont celles des 149 tiges, de 1 à 149 ans dans notre exemple et, en outre, celles de tout le remplissage. Que celles-ci soient utilisées à une exploitation intermédiaire ou qu'elles ne le soient pas (peu importe, du moment où elles pourraient l'être), elles forment des récoltes en croissance de cette exploitation composée.

Il faut y joindre le matériel de l'exploitation périodique, c'est-à dire le semis initial de toutes les tiges principales de $\bar{l}$ à 150 ans par exemple ( $f g .5$ ) et les semis de toutes les tiges de remplissage, semis qui se touchent, qui sont lents à se développer et qui occupent, avec celui des tiges principales, toute l'étendue du terrain consacré à l'exploitation ( fig. 6).

La forêt furetée est exploitée à trop court terme pour donner place à des produits intermédiaires. Si le propriétaire coupe parfois quelques brins inférieurs à la dimension adoptée, c'est dans un but uniquement cultural, pour favoriser des brins d'avenir et non pour constituer une exploitation composée digne de ce nom.

La futaie étagée forme un type très complet d'exploitation aménagée composée, dont les arbres sont isolés yar la main de l'homme. 
Réunissez 28 hectares constitués comme celui à exploitation périodique qui est indiqué $n^{\circ} 77$, vous obtiendrez une exploitation à revenu annuel : en consacrant à la coupe un hectare chaque année, de manière à revenir tous les 28 ans sur le même hectare, la forêt fournira chaque année 120 tiges de diverses dimensions variant de $0^{\mathrm{m}}, 10$ à $0^{\mathrm{m}}, 60$ de diamètre, c'est-à-dire ayant des âges divers, espacés entre eux de 28 ans.

Le matériel d'exploitation est formé, dans ces forêts, de toute la superficie boisée, à laquelle il faut joindre le semis initial sur toute la surface, en supposant, bien entendu, que tous les hectares se ressemblent pour le nombre des arbres, comme cela doit être dans toute conception théorique.

Dans l'exemple précédent, le matériel serait de 28 fois 390 tiges, soit 10,920 arbres, nécessaires pour assurer la perpétuité du revenu annuel ; en effet, l'analyse des six exploitations annuelles d'arbres isolés montre que la forêt devra présenter :

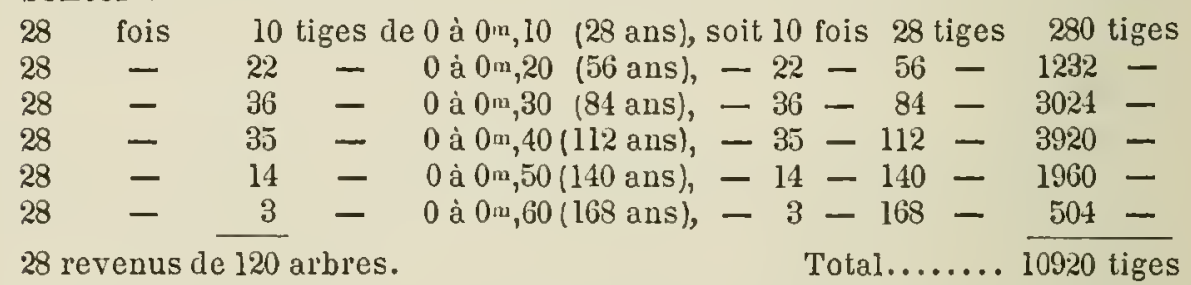

Ces forêts sont très avantageuses pour les petites propriétés où l'exploitant veut produire à la fois des arbres de grand âge et des arbres d'âges divers. C'est à la condition, toutefois, que l'exploitation soit pratiquée sur des terrains et en des climats convenables avec des essences appropriées à ce mode de traitement.

Le matériel de cette exploitation est un des plus considérables qu'on puisse accumuler sur une contenance déterminée, aussi l'augmentation du capital produit ses effets, et ces forêts sont les plus riches en produits, les meilleurs et les plus utiles; elles ont le plus haut revenu annuel en argent.

Dans l'exemple précédent, la coupe de 120 arbres peut être 
évaluée de la manière suivante, en estimant les hêtres qui peuplent la forèt aux prix constatés dans une forêt du sud de la Belgique, en 1875.

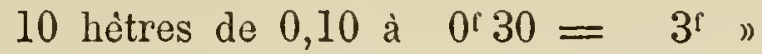

$$
\begin{aligned}
& 22-0,20 \text { à } 2 \text { - }=44 \text { " } \\
& 36-0,30 \text { а̀ } 8 \text { " } \quad 0,40 \text { ว } \\
& 35-0,40 \text { à } 22 »=770 \text { » } \\
& 14-0,50 \text { à } 42 »=588 \text { » }
\end{aligned}
$$

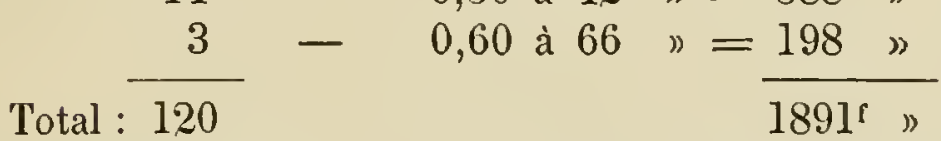

Les 28 hectares qui forment cette forêt rapportent ainsi en moyenne 65 francs par hectare et par an.

$\mathrm{N}^{\circ}$ 82. - L'exploitation aménagée de peuplements d'un seul âge est composée quand elle fournit chaque année des produits intermédiaires dus aux coupes d'éclaircies.

La figure 17 représente une exploitation de peuplements, aménagée à 120 ans sur 120 hectares; les éclaircies faites à 30 , 60 et 90 ans sont figurées en noir comme la coupe principale à 120 ans. Les hachures perpendiculaires expriment le nombre d'arbres décroissant dans chaque peuplement à mesure que l'âge s'approche de 120 ans.

Nous avons indiqué ( $n^{\circ} 78$ ) l'idée culturale sur laquelle est fondée la pratique des éclaircies et nous avons vu qu'il n'existe pas de matériel spécial pour les coupes d'éclaircies comme il en existe pour les exploitations d'arbres isolés par la main de l'homme; mais c'est à la condition que les peuplements seront comptés pour le volume et la valeur qu'ils ont à chaque âge.

Le matériel-bois de cette exploitation est donc formé, comme dans l'exploitation simple, par la suite des coupes en croissance depuis l'âge du début jusqu'à celui assigné pour terme à l'exploitation. Il faut y joindre le semis ou l'ensouchement sur toute l'étendue de la forêt; il en résulte que ce matériel est formé par la suite des récoltes pendantes et par le matériel-bois de l'exploitation périodique. 
Dans l'exemple de la figure 17, les coupes d'éclaircies sont annuelles; elles se font chaque année sur un hectare, au fur et à mesure que les époques fixées (30, 60, 90 ans) sont successivement atteintes. Cela suppose que la forêt a une étendue assez grande pour livrer des coupes faciles à vendre. Dans le cas contraire, on remédie à la difficulté de vendre de trop petites coupes, par des combinaisons multiples dont il suffit d'indiquer deux types:

Les éclaircies de 30, de 60 et de 90 ans sont, par exemple, biennales et pratiquées sur 2 hectares tous les 2 ans.

On peut aussi grouper les peuplements en quatre quartiers ou affectations ainsi qu'il suit:

$\begin{array}{cccccc}\text { Quartier des jeunesses... } & \text { de } 1 \text { à } 30 \text { ans.. } & 30 \text { hectares. } \\ \text { - des perchis..... } & \text { de } 30 \text { à } 60 \text { ans.. } & 30 & \text { - } \\ \text { - des jeunes futaies } & \text { de } 60 \text { à } 90 \text { ans.. } & 30 & \text { - } \\ \text { - de la futaie..... } & \text { de } 90 \text { à } 120 \text { ans.. } & 30 & \text { - }\end{array}$

120 hectares.

Pendant que le quartier le plus âgé fournit la coupe principale en bois de 120 ans, les quartiers affectés aux coupes intermédiaires fournissent par exemple :

Le premier: des nettoiements culturaux de 5 hectares par an pour revenir tous les 6 ans.

Le second : des premières éclaircies de 3 hectares par an espacées de 10 ans.

Le troisième : des deuxièmes éclaircies de 3 hectares par an revenant sur le même point tous les 10 ans.

Quelles que soient ces combinaisons, la conception théorique de l'exploitation est toujours la même.

Les forêts de peuplements d'un seul âge ne conviennent qu'à de grands propriétaires quand elles sont réglées à un âge avancé; il faut en effet les installer sur des étendues assez considérables pour qu'une surface suffisante soit affectée à l'éducation et à la culture des peuplements de chaque âge. Que les peuplements d'âges roisins soient groupés sur des cantons qui leur sont spécialement affec- 
tés (affectation), cela importe peu, il n'en faut pas moins que l'échelle complète des âges existe sans interruption sur des contenances égales pour chaque âge. On ne saurait, en effet, couper tous les ans un hectare de 120 ans, par exemple, qu'à la condition de posséder 120 hectares, ayant tous les âges compris entre 1 et 120 ans.

No 83. - Les exploitations aménagées mixtes, d'arbres et de peuplements, peuvent être doublement composées, c'est-à-dire que la forêt de peuplements peut fournir un revenu intermédiaire en éclaircies et que la forêt d'arbres qui lui est superposée peut produire, également chaque année, des arbres de différentes dimensions.

Les forêts de taillis sous futaie composée offrent le plus souvent une exploitation simple pour le taillis et une exploitation composée pour les arbres de la futaie. Celle-ci mérite d'être analy'sée pour déterminer son matériel.

Sur une forêt de 30 hectares dont le taillis a été réglé à 30 ans, on veut avoir tous les ans un revenu de:

$$
\begin{aligned}
& 20 \text { modernes de } 60 \text { ans } \\
& 30 \text { anciens de } 90 \text { ans } \\
& 10 \text { bis-anciens de } 120 \text { ans } \\
& \text { Total. } \quad \frac{60 \text { arbres. }}{}
\end{aligned}
$$

Cela correspond à un plan de balirage composé de $110 \mathrm{ar}-$ bres.

$$
\begin{aligned}
& 60 \text { baliveaux, } \\
& 40 \text { modernes, } \\
& 10 \text { anciens. }
\end{aligned}
$$

Pour se rendre compte du matériel d'exploitation, il faut considérer la forêt comme réunissant deux exploitations superposées, celle du taillis et celle des arbres.Ceuxci forment, par dessus le taillis, trois exploitations juxtaposées.

Celle des modernes;

Celle des anciens;

Celle des bis-anciens. 
Si nous raisonnons sur un arbre de revenu, nous voyons que :

L'exploitation des modernes comporte une suite de 60 arbres de 1 à 60 ans;

Celle des anciens, une suite de 90 arbres de 1 à 90 ans ;

Celle des bis-anciens, une suite de 120 arbres de 1 à 120 ans.

Comme l'exploitant ne possède que 30 coupons (puisque le taillis est réglé à 30 ans), il est obligé de disposer ces suites d'âges sur ses 30 coupons.

La suite des modernes en deux parties:

de 0 à 30 ans sur les coupons de 1 à 30

de 30 à 60 ans _ $\quad 1$ à 30

La suite des anciens forme trois parties:

de 0 à 30 ans sur les coupons de 1 à 30

de 30 à 60 ans - $\quad 1$ à 30

de 60 à 90 ans _ $\quad 1$ à 30

La suite des bis-anciens a quatre parties:

de 0 à 30 ans sur les coupons de 1 à 30

de 30 à 60 ans _ $\quad 1$ à 30

de 60 à 90 ans - $\quad 1$ à 30

de 90 à 120 ans - $\quad 1$ à 30

Cette disposition est indiquée à la figure 18 .

Remarquons, d'abord, que la première partie de ces trois suites, celle de 0 à 30 , est noyée dans le taillis ; elle n'est pas encore dégagée; l'isolement des tiges ne commence que pour les arbres de 30 ans et au delà.

La $1^{\text {ro }}$ suite est réduite à 1 partie,

La $2^{\circ} \quad-\quad 2$ parties,

La $3^{\circ} \quad-\quad 3$ parties.

Il en résulte que pour exploiter un bis-ancien, il faut qu'il y ait sur la coupe mûre, en outre de cet arbre, un ancien et un moderne ( 3 arbres).

Pour exploiter chaque année un ancien, il faut qu'il y ait, en outre, un moderne ( 2 arbres).

Pour couper annuellement un moderne, il suffit de cet arbre (1 arbre). 
Si on veut couper chaque année, à la fois, un bis-ancien, un ancien et un moderne, il faudra que la coupe mûre présente 6 arbres.

\section{3 modernes ; \\ 2 anciens; \\ 1 bis-ancien.}

Le revenu est, en effet, formé par la différence entre les arbres de chaque catégorie.

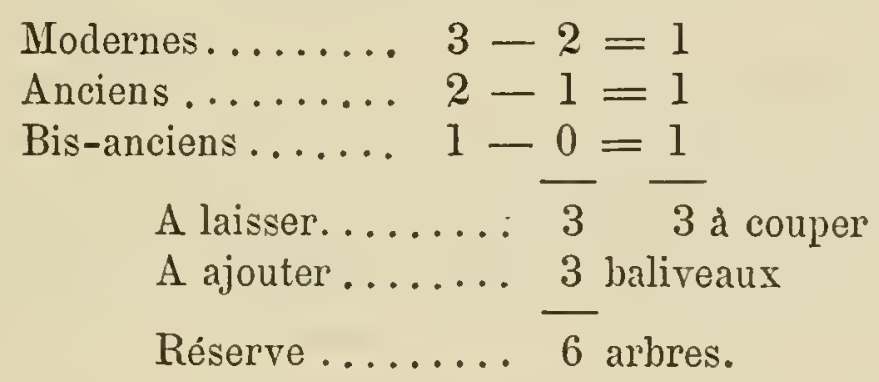

Cette exploitation en apparence compliquée s'effectue de la manière la plus simple: il suffit de s'imposer pour règle de conduite de réserver chaque année un plan de balivage constant qui est dans notre exemple :

$$
\begin{aligned}
& 60 \text { baliveaux, } \\
& 40 \text { modernes, } \\
& 10 \text { anciens. }
\end{aligned}
$$

Ce plan permettra d'avoir toujours un revenu de 60 arbres:

$$
\begin{aligned}
60-40 & =20 \text { modernes } \\
40-10 & =30 \text { anciens } \\
10-0 & =\frac{10}{60} \text { bis-anciens. }
\end{aligned}
$$

On voit qu'on ne coupe jamais de baliveaux, ce sont des arbres de remplacement pris dans le taillis.

Le nombre des baliveaux est égal au nombre total des arbres coupés en revenu, sauf la part que l'on doit faire dans la pratique au déchet qui peut survenir.

Le nombre des baliveaux est égal au nombre des modernes, parce que tous doivent arriver à cette catégorie. 
Mais tous les modernes ne devenant pas anciens, leur nombre est égal à celui des anciens qu'on réserve, augmenté du nombre des bis-anciens.

Quant au nombre des anciens, il est égal à celui des bis-anciens si on limite à cette catégorie le terme extrême de l'exploitation.

La formule des anciens règlements de jouissance: " laisser sur chaque hectare de coupe 60 baliveaux, 40 modernes et 10 anciens ", est ce qu'on appelle le plan de bativage. Nous venons de voir qu'il suffit de connaître le plan de balivage pour en déduire le revenu par un simple et rapide calcul qui correspond à la règle suivante: Le revenu de la futaie composée est égal à la somme des différences qui existent entre chaque classe d'arbres sur la coupe mure.

Le plan de balivage permet aussi de déterminer le matériel d'exploitation. Pour obtenir le nombre d'arbres dont on doit garnir le terrain pour assurer l'exploitation de la futaie, il suffit de muttiplier le plan de balivage par la contenance.

Dans l'exemple précédent:

$$
110 \times 30=3300 \text { arbres. }
$$

Les arbres sont ainsi détaillés:

60 baliveaux passant aux modernes...... $60 \times 30=1800$ de 30 à 60 ans. 10 modernes - anciens ......4 $40 \times 30=1200$ de 60 à 90 ans. 10 anciens $\quad-\quad$ bis-anciens.... $10 \times 30=300$ de 90 à 120 ans. Total..... 3300

On arrive au même résultat en considérant le revenu déduit du plan de balivage. Le matériel de chaque exploitation est :

Modernes. . 20 groupes de 30 tiges de 30 à 60 ans $=600$ Auciens... $30-60-30$ à 90 ans $=1800$ Bis-anciens $10-90-30$ à 120 ans $=900$

Total......... $\overline{3300}$

Ainsi, dans les forêts de taillis sous futaie composée, le plan de balivage fournit, à la fois et par une opération très simple, le revenu et le matériel-bois de l'exploita- 
tion. Il en résulte que dans les exploitations mixtes aménagées, le matériel-bois est composé du matériel de l'exploitation en taillis et de celui de l'exploitation en arbres. Il est facile d'évaluer le premier puisque les arbres occupent sur la surface une quantité constante déterminée par leur couvert ( $f g .5)$. Quant au matériel des arbres, il est facile également d'en estimer la valeur quand on l'a détaillé comme nous l'avons fait ci-dessus à l'aide clu plan de balivage.

La figure 18 montre la disposition des 30 coupes qui constituent l'exploitation. La ligne de 0 à 30 représente le taillis; quant aux arbres, ils sont figurés par des groupes de lignes verticales dont la hauteur exprime les classes d'âges et dont l'épaisseur est proportionnelle à la valeur' que les arbres prennent en vieillissant.

Lorsqu'on convient de représenter chaque classe par des lignes ascendantes parallèles à celles du taillis et le nombre d'ar'bres dans chaque classe par une bande placée sous chaque ligne ascendante, on obtient la figure 19 qui reprèsente plus simplement l'exploitation .

Lor'squ'enfin, on convient de représenter les classes d'âges par des rectangles dont la hauteur est proportionnelle à l'âge et dont la largeur exprime le nombre de chaque catégorie, on obtient la figure 20 qui montre la composition intime de l'exploitation.

Ces diagrammes confirment la remarque déjà faite pour les exploitations aménagées: le matériel d'exploitation comprend : $1^{\circ}$ la suite des récoltes pendantes en taillis et en arbres; $2^{\circ}$ le matériel de l'exploitation périodique en taillis et en arbres sur toute la surface. Les parties blanches de la figure 20 représentent ces récoltes pendantes et les parties noires le matériel initial.

No 84. - Exploitations graduées. - Elles se prêtent, comme celles périodiques et aménagées, à l'état composé et cela dans chaque mode de traitement des forêts.

Ainsi 30 hectares de taillis sous futaie composee peuvent 
être divisés en 10 lots pour en couper un chaque trois ans de la même manière que le taillis ordinaire.

Dans les forêts de peuplements, la perception des produits intermédiaires peut alterner avec celle des produits principaux.

20 hectares de pineraie exploités à 40 ans à raison de 1 hectare tous les 2 ans peuvent fournir également une éclaircie biennale de 2 hectares à l'âge de 20 ans. Si la coupe principale est faite aux millésimes pairs et l'éclaircie aux millésimes impairs, le propriétaire de cette petite forêt aura par cette combinaison l'avantage de mieux répartir ses ressources et d'aller chaque année visiter et soigner son exploitation.

Des exploitations aménagées peuvent se constituer avec des éléments d'exploitations graduées $\left(\mathrm{n}^{\circ} 75\right)$ et fournir chaque année des produits composés.

30 hectares de sapinière jardinée peuvent fournir 36 sapins par an à raison d'un arbre et un cinquième par hectare par an. Cette exploitation est disposee en 10 coupons de 3 hectares chacune et fournissant chacun, à tour de rôle, les 36 sapins par an. Cinq ans après la coupe, le propriétaire recueille 60 pannes sur le coupon exploité, de telle sorte que chaque coupon fournit successivement 36 sapins et 60 faibles tiges, chaque coupe étant à intervalle de 10 ans et se suivant à la distance de $\breve{b}$ ans.

Cette exploitation est exactement aménagée en ce sens qu'elle fournit, en revenu annuel, aussi bien la coupe principale que la coupe intermédiaire. Mais on craint que la petite quantité d'arbres ne soit difficile à vendre et que la forêt ne se fatigue par ces coupes répétées tous les cinq ans sur le même coupon.

On admet alors la combinaison suivante. Chaque coupon de numéro impair fournit aux millésimes impairs la double production principale de 72 sapins, et chaque coupon de numéro pair fournit aux millésimes pairs la double production intermédiaire de 120 petites charpentes; mais au bout de 10 ans, quand les 10 coupons ont été parcourus, on a soin de les reprendre toujours dans le même sens, mais dans l'ordre con- 
traire, les coupons pairs pour la coupe principale, ceux impairs pour la coupe intelmédiaire. De cette manière, chaque coupon fournit la double nature de production mais se repose pendant 10 ans .

C'est une exploitation graduée à produits composés alternants.

On aurait pu également diviser l'immeuble en 5 coupons de 6 hectares à rotation de 10 ans, chacun fournissant successivement $\% 2$ sapins tous les 2 ans et, en même temps, 120 arbres de petite charpente.

Dans cette exploitation graduée à perception composée biennale, les coupons se reposent pendant 10 ans; ils sont fatigués par la perception des deux doubles productions principale et intermédiaire; mais cette perception s'opère sur une surface double.

On conçoit combien les combinaisons d'exploitation sont nombreuses et variées. Cet exemple suffit à en indiquer les ressources. La forêt se prête à tous les arrangements de fortune et à tous les plans de conduite; mais c'est à la condition de respecter toutes les exigences culturales des essences et de leur mode de traitement.

\section{$\S 4 .-$ Observations.}

Nos 85̆. - Remarques générales sur le capital d'exploitation des forêts.

86. - Comparaisons arec les exploitations agricoles.

87. - Utilité de la notion du capital d'exploitation.

88. - Portée de la conception théorique; forèt normale.

№ 85. - On ne produit rien avec rien. Cette vérité économique de toutes les entreprises humaines recoit són application dans les forêts considérées comme exploitations à bois, mais l'effet du capital s'y présente avec des particularités dignes de plusieurs remarques.

$1^{\circ}$ L'augmentation du capital-bois n'accroît pas la quan- 
tité des produits qui sont dus à la seule action des agents naturels, mais elle a pour effel de fournir des produits plus âgés, c'est-à-dire de qualité meilleure et de les livrer à des intervalles plus rapprochés.

C'est ainsi qu'une forêt garnie d'arbres épargnés et conservés en vue d'une production future permet de recueillir tous les 30 ans des arbres de 60,90 et 120 ans, selon la qualité de la réserve $\left(\mathrm{n}^{\circ} 80\right)$.

L'intervalle entre la jouissance des coupes se rapproche de plus en plus et la perception finit par être annuelle, quand le capital d'exploitation atteint son maximum dans la forêt aménagée.

$2^{\circ}$ Lorsqu'on examine la constitution intime du capital d'exploitation, on voit que dans les forêts aménagées, ce capital est formé de deux éléments : $1^{\circ}$ Te matériel initial de l'exploitation périodique; 20 la suite des récoltes pendantes depuis l'âge du début jusqu'à celui qui est assigné pour terme à l'exploitation. Le matériel initial existe dans toute les espèces d'exploitations; la quantité de récoltes pendantes diminue dans les exploitations graduées à mesure que l'intervalle des coupes augmente.

$3^{\circ}$ Le matériel d'exploitation étant toujours vivant, se renouvelle et s'entretient seul, sans que l'exploitant ait souci des dépenses et des soins de l'entretien. Il s'accroît constamment par l'effet de la vie, mais chaque coupe le ramène à son état primitif, de telle sorte que l'exploitant sait toujours de quelle somme il a doté son exploitation, sans qu'il ait à compter aucun amortissement pour le ramener à son état primitif par un prélèvement sur le revenu. Cet avantage n'existe dans aucune entreprise; dans toutes le matériel s'use, a besoin de réparations et d'une surveillance constante pour le maintenir à sa valeur primitive.

$4^{\circ}$ Dans toute exploitation forestière, le matériel d'exploitation est maintenu constant et invariable, tant que le 
but de l'exploitation, c'est-à-dire l'âge de la coupe, n'a pas été changé. Il varie seulement d'emplacement et de disposition à mesure que les coupes avancent pour revenir à la disposition du début après la dernière coupe. La figure 21 montre l'état du matériel d'un taillis ordinaire aménagé à 30 ans au moment de la dixième coupe. On voit que le matériel initial $A B$ a pris une disposition différente, mais entièrement équivalente, et que dans 20 ans il reviendra à l'arrangement du début.

$5^{\circ}$ De ce que le produit annuel de l'exploitation forestière est de la même nature que son capital-bois, il résulte que la confusion est toujours possible et que l'exploitant est souvent exposé à aller au delà de son revenu en entamant son capital. C'est ce qu'on s'efforce d'empêcher par l'assiette de l'aménagement, c'est-à-dire par la division de la forêt en coupes assises et marquées sur le terrain. C'est dans ce but, également, qu'une administration publique est chargée de veiller à ce que la jouissance du présent dans les forêts de l'État et des communes n'empiète pas sur le matériel-bois qui est la garantie des revenus de l'avenir.

$6^{\circ}$ Enfin, au point de vue juridique, le capital d'exploitation des forêts est toujours immeuble (C. civ. 520) et mobilisable (C. civ. 521), parce qu'il est composé d'arbres sur pied. Il en résulte que le crédit liypothécaire est toujours restreint aux propriétaires de forêts et que l'organisation du crédit agricole mobilier leur sera aussi utile

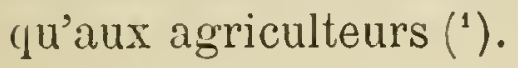

$\mathrm{N}^{\circ}$ 86. - Il n'est pas sans intérêt de comparer l'exploitation forestière aux exploitations agricoles.

$1^{\circ}$ Dans celles-ci le capital nécessaire à la production est formé par les bâtiments de la ferme, par les engrais, les

( ${ }^{1}$ Voir A. Puton, "les Forèts et le Crédit agricole ", Revue des eanx et foréts, 1883. 
semences, le bétail et les instruments. Il est en partie immeuble, en partie meubles immobilisés par destination (C. cir. 524). Mais tout ce matériel se dégrade, s'use et a besoin d'être réparé ou remplacé. L'agriculteur prélève par exemple 10 0/0 sur ses revenus pour l'entretenir. Ce prélèvement fait et employé, l'exploitation est maintenue dans le même et identique état. Si le propriétaire de forêts juge utile d'épargner 10 0/0 sur son revenu et de laisser le dixième de la coupe sans le réaliser, il amétiore, au contraire, son exploitation et en percoit des produits plus âgés et plus avantageux.

Une forêt de 30 hectares exploitée à 30 ans par exemple, et produisant 150 mètres cubes, se trouve réglée à 33 ans par un prélèvement du dixième; elle continue à fournir 150 mètres cubes par an, mais en bois meilleur et se vendant plus cher.

$2^{\circ}$ En agriculture, si l'exploitant fait mal ses affaires ou si des circonstances l'obligent à liquider, il ne peut généralement vendre les bâtiments sans la terre elle-même, car sans elle, ils n'auraient pas de valeur. L'exploitant forestier peut, au contraire, faire argent de tout le capital bois qui se trouve sur la propriété et alor's la terre reste sans livrer de produits pendant longtemps, pendant tout le temps qui est nécessaire à la nature pour reconstituer du bois exploitable. Ceci explique comment il est nécessaire qu'une administration publique veille à la conservation du matériel d'exploitation dans les forêts de l'Etat et dans celles des communés et des établissements publics; car ces êtres moraux ne sont dans l'ordre social que des usufruitiers et doivent transmettre aux générations futures la propriété munie de son matériel complet. L'intérêt public aurait trop à souffrir si pour une circonstance mêrne très urgente, on venait à l'entamer d'une manière générale; car un pareil capital ne se reforme pas avec de l'argent comme les bâtiments d'une ferme, mais par le temps seul et par un temps souvent fort long. 
$3^{\circ}$ Notons encore une autre différence économique entre le capital d'une exploitation agricole et le matériel destiné à l'exploitation d'une forêt aménagée : en agriculture, le propriétaire qui a besoin d'argent peut vendre son bétail, ses charrues, ses voitures. Généralement ces instruments d'exploitation sont achetés par des cultivateurs et vont servir à monter une autre exploitation agricole: un individu peut être gêné, mais la société n'en souffre pas.

Le propriétaire d'une forêt, au contraire, est toujours tenté, quand de mauvais jours surivennent, de vendre une partie des bois qui forment l'échelle d'âges nécessaire à une production annuelle, et il arrive un double malheur : Le premier est que le matériel vendu ne va pas à une autre exploitation; il y a diminution dans le nombre des outils qui travaillent à fournir au pays la production ligneuse. Le second vient de ce que la portion de bois prise sur le matériel d'exploitation étant vendue en chauffage ou en bois de service, l'offre de la marchandise peut dépasser la demande. La production peut n'être plus en rapport avec la consommation; et, les prix s'avilissant, l'intérêt public souffre, parce que les propriétaires voisins n'obtiennent plus un prix rémunérateur, parce que les métiers qui usent du bois sont un instant dans l'abondance pour le payer plus tard trop cher, quand la rareté sera venue, parce que, enfin, une denrée précieuse, en ce sens que le temps seul et non la main des hommes, en est l'ouvrier, a été avilie et gaspillée.

$N^{\circ} 87$. - Nous nous sommes étendu sur la conception théorique de l'exploitation et sur la constitution de son capital, parce que cette notion est fondamentale et nécessaire aux propriétaires pour se rendre compte de tous leurs actes de gestion. Quelle est l'influence d'un balivage, maintiendra-t-il la forêt dans le même état, dimi- 
nuera-t-il ou en accroîtrera-t-il sa valeur et dans quelle mesure la diminution ou l'angmentation seront-elles produites? Le propriétaire ne peut s'en rendre compte que par la connaissance de la manière dont son exploitation est constituée; il en est de même des anticipations et des retards de coupe, de la prolongation ou de l'abaissement de l'âg'e auquel il exploite.

La notion du capital d'exploitation est, en outre, indispensable pour la solution de toutes les questions les plus importantes de l'économie forestière. Seule, elle permet d'en raisonner avec méthode et sûreté. Nous nous bornons à la remarque suivante:

Nous avons établi comme règle générale des exploitations aménagées: que le matériel est égal à celui de l'exploitation périodique augmenté des récoltes pendantes depuis l'âge du début jusqu'à celui de la coupe. Cette règle montre que l'exploitation aménagée est formée d'éléments à exploitations périodiques; que les règles de l'une seront applicables à l'autre et que dans tous les problèmes d'économie forestière une solution ne sera vraie qu’à la condition de s'appliquer à l'une et à l'autre avec le même raisonnement.

La notion du capital donne, en outre, la clef des problèmes les plus délicats de l'estimation des forêts. C'est par elle qu'on est en droit de dire, que dans l'expropriation pour cause d'utilité publique on doit à l'exproprié "la valeur du sol gami de son matériel d'exploitation, plus la récolte pendante escomptée au prix que le propriétaire expropriéla rendrait à son échéance (') ).

C'est ainsi encore que dans la question si contestée de la liquidation des droits d'usag'e, on peut établir en principe que "l'usager absorbant tous les fruits a droit à la valeur de toutes les récoltes pendantes et le propriétaire à

( ${ }^{1}$ Estimations concernamt la propriété forestière, $1886, n^{05} 50$ à 52 
la valenir du sol garni du matériel de l'exploitation périodique. L'usager qui n'absorbe qu'une fraction du revenu n'a droit qu'à la même fraction des récoltes pendantes ( $\left.{ }^{1}\right)$ ).

Les anciens forestiers n'avaient aucune notion sur la constitution des exploitations des forêts et sur le rôle du capital. La science de l'économie politique date à peine du siècle dernier. Il est temps que ses principes et ses données s'introduisent dans l'économie forestière $\left({ }^{9}\right)$.

$\mathrm{N}^{\circ} 88$. - La conception du matériel d'exploitation est entièrement théorique en ce sens que son application stricte aux forêts n'est pas réalisable d'une manière soutenue. Les forêts les mieux traitées et les mieux aménagées ne se présentent jamais avec la régularité absolue de la théorie. C'est à peine si cette régularité se rencontre dans quelques petites forêts de taillis exploitées à court terme par des locataires. Il serait sans raison de s'épuiser en efforts pour atteindre le type théoriquement conçu, tant les besoins de la vie et les inégalités du sol ou des essences peuvent y apporter d'obstacles; mais on saura au moins vers quel but on tend et de quelle manière on s'en écarte.

On a donné le nom de forêt normale à la conception théorique de l'exploitation amėnagée; nous pensous qu'on peut se passer non de l'idée, mais du mot, parce que celui-ci peut faire naitre la pensée d'une règle immuable et unique imposée à la conduite et aux efforts du forestier. Si cette forêt normale exprime une conception théorique de l'exploitation, il doit $\mathrm{J}$ avoir autant de forèts normales que de modes de traitement et de combinaisons d'exploitation dans chaque mode de traitement; le mot devient alors inutile. Si la forêt normale implique l'idée

(1) Estimations, nos 88 à 91 .

$\left({ }^{2}\right)$ La notion du capital d'exploitation empruntée à l'économie politique générale a été introduite par nous, pour la première fois, en 1867, daus la science forestière: A. Puton, l'Aménagement des torêts, 1 rol. ill-12, 3e ćdition, 1883. 
d'un mode d'exploitation par excellence, devant s'appliquer partout comme la règle unique du traitement des forêts, l'idée est fausse et doit être abandonnée.

Il y a, en forêt, comme en toute exploitation, des approximations qu'il faut savoir comprendre et qui sont dans la nature des choses; l'essentiel est que l'exploitant connaisse bien le but de son exploitation, sa constitution et ses organes, pour qu'il puisse en discuter sûrement.

Quant à l'économiste, il ne raisonne que sur l'idée théorique et sur la conception faite en vue d'arriver à un résultat donné. Ce n'est pas qu'il néglige les faits ni leur application, mais c'est seulement quand la théorie est établie et concue qu'il doit s'occuper des forêts telles qu'elles se rencontrent habituellement.

Un traité d'économie forestière doit suivre cette marche ; il doit indiquer d'abord la conception théorique et ensuite l'application de la théorie aux faits, l'aménagement d'une forêt réelle, c'est-à-dire les procédés employés pour installer une exploitation aménagée suivant un but donné dans une forêt, telle qu'elle se rencontre réellement et pratiquement. 


\section{AGE D'EXPLOITATION}

Nos 89. - But de l'exploitation. - Révolution.

90. - Problèmes sur l'àge d'exploitation. - Exploitabilité.

91. - Remarques sur ces problèmes.

92. - Définition de la futaie.

93. - Exploitation intensive et extensive.

94. - Comparaison avec les exploitations agricoles.

95. - Fpargne nécessaire pour rendre une exploitation intensive.

96. - Conversion et transformation.

No 89. - Toute exploitation a un but général et un but spécial. Le but général des exploitations forestières est de produire du bois, et l'on aurait bien tort de chercher à la fois la production du bois et celle du pâturage; ce sont deux productions qui s'excluent et se détruisent mutuellement : on ne saurait tirer deux moutures du même sac de blé.

Mais la matière ligneuse a différents degrés d'utilité et de cherté suivant la grosseur, c'est-à-dire suivant l'âge de l'arbre qui produit le bois. Le but spécial de l'exploitation est de produire des tiges capables de fournir des bois déterminés. Tel propriétaire veut faire produire à son taillis des bois capables de servir d'étais de mines; tel autre veut régler l'exploitation d'une sapinière de manière à obtenir des arbres de sciage; un autre se contente de bois de charpente ou de perches télégraphiques; un autre, enfin, demande à sa forêt des bois de dimensions variées. A un autre point de vue, certains propriétaires veulent obtenir des fonds engagés un taux de placement déterminé, 3 p. $0 / 0$ par exemple. 
Le but de l'exploitation peut donc être très différent suivant les conditions ou elle se trouve placée et suivant la fortune du propriétaire. Ce but se manifeste par l'âge auquel on règle l'exploitation.

L'âge ou le terme de l'exploitation est encore le temps pendant lequel le matériel d'exploitation a été consommé et remplacé par un nouveau. Ainsi, dans une forêt de taillis aménagée à 35 ans, cet âge indique à la fois qu'on produit des bois de 35 ans (étais de mines par exemple) et que les 35 coupes amuelles seront consommées et remplacées en 35 ans.

On a appelé révolution le nombre d'annẻes déterminé pour l'exploitation d'une forêt $\left({ }^{1}\right)$. Si on exploite un bois à 35 ans, la révolution est de 35 ans. La révolution est donc une expression synonyme de l'âge d'exploitation dont le besoin ne se faisait nullement sentir $\left({ }^{2}\right)$.

Si par le mot révolution, on entend exprimer que la coupe reviendra en 35 ans an point de départ, cela est inexact pour certaines forêts, par exemple, pour celles aménagées en jardinage avec une rotation au bout de laquelle la coupe revient sur le coupon du début.

Si enfin, la révolution exprime le temps pendant lequel on aura consommé le matériel d'exploitation, la même idée se trouve renfermée dans le terme d'exploitation et deux mots sont bien inutiles pour exprimer la même chose.

On peut écrire tout un livre d'économie forestière sans employer une seule fois le mot révolution qui était inconnu des anciens forestiers; c'est la meilleure preuve qu'on puisse domner de son inutilité.

L’âge est ainsi la note caractéristique de l'exploitation; il exprime son but, la dimension des bois, leur utilité, leur volume et leur valeur. Il en résulte que dans nos diagrammes, l'âge suffit à exprimer le volume et la valeur

(1) Lorentz et Parade, Culture des bois, 6e édit., p. 151.

$\left.i^{2}\right)$ Le mot révolution ne se trouve pas dans le Dictionnaire de Chailland ni même dans celui de Baudrillart. 
des peuplements; il sert de base aux calculs de l'usufruit et aux méthodes de partage ( $\left.{ }^{1}\right)$.

$\mathrm{N}^{\circ}$ 90. - Il y a autant de buts à une exploitation de forêt qu'il y a de différences dans la situation des propriétaires et de conceptions dans leurs combinaisons. De là, un certain nombre de problèmes sur l'âge de l'exploitation qui s'imposent à l'étude de l'économie forestière. Nous ne pouvons les énumérer tous, tant sont multiples les buts poursuivis. Nous indiquerons cependant les principaux dans l'énumération suivante:

A quel âge faut-il exploiter une forêt?

$1^{\circ}$ Pour que la hauteur des tiges ne gêne pas le tir d'une place forte ou la vue d'une habitation, ou bien pour que le massif forme un rideau protecteur contre des vents dangereux;

$2^{\circ}$ Pour que la forêt produise des bois propres à fournir une marchandise déterminée, par exemple: des étais de mine, ou de la charpente, ou du sciage;

$3^{\circ}$ Pour que les arbres fournissent les marchandises les plus variées et les plus utiles;

$4^{\circ}$ Pour que la production ligneuse soit la plus considérable possible;

$5^{\circ}$ Pour que le produit en argent soit le plus élevé possible;

$6^{\circ}$ Pour que le propriétaire obtienne des fonds engagés dans l'exploitation le taux de placement le plus élevé possible ;

$7^{\circ}$ Pour que le propriétaire ait ses fonds engagés dans l'exploitation placés à un taux déterminé, par exemple celui de $30 / 0$ usité dans la localité pour les placements en immeubles agricoles.

Ces questions ont reçu de certains auteurs le nom de pro-

( $\left.{ }^{1}\right)$ Estimations concernant la propriété forestière, 1 vol. grand in- $8^{\circ}, 1886$, nos 123 et suivants. 
blèmes d'exploitabilité. Ce mot inconnu des anciens forestiers et même de ceux qui ont écrit, comme Baudrillard, au début $\mathrm{du}$ siecle, offre à l'esprit une idée peu nette. Si, en effet, l'ex ploitabilité sert à désigner la qualité, l'aptitude à être exploité comme en pliysique la fusibilité, la conductibilité expriment certaines qualités des corps, il faut convenir que l'exploitabilité ne signifie pas grand chose, puisque tous les bois sont aptes à être coupés dès l'âge le plus tendre jusqu'à l'âge le plus avancé.

$\mathrm{Si}$, au contraire, ce mot signifie le but, la condition assignée à l'exploitation, il faut alors lui ajouter un déterminatif comme on fait en Droit pour la condition potestative, résolutoire, etc. C'est effectivement ce qui a été pratiqué et on a distingué les exploitabilités physique, technique, économique, composée, absolue, relative, financière, commerciale, etc. Mais alors, il faut toujours avoir à l'esprit ce que signifient ces mots, et quel problème a été ainsi dénommé. Il est arrivé que certains auteurs ayant cru mieux faire que leurs devanciers, ont donné au même mot des sens différents. La confusion s'est introduite dans le langage forestier. Il nous paraît beaucoup plus simple de désigner un problème d'exploitabilité par son énoncé, que de lui donner un nom arbitraire dont l'esprit demande l'explication des que l'oreille l'entend. Nous préférons exploitabilité offrant le taux de placement des immeubles agricoles à exploitabilité commerciale, parce que ce mot appelle une définition sans laquelle on est exposé à ne pas s'entendre.

Nous renonçons à désigner ces diffẻrents problèmes sur l'âge d'exploitation par un mot spécifique même emprunté aux auteurs forestiers, car, outre le motif de simplification que nous venons d'indiquer, on ne saurait dissimuler qu'il y a de nombreuses variantes dans les dénominations usitées. Pour être juste, il faudrait presque toujours ajouter le nom de l'auteur à la terminologie et indiquer les synonymes, ce qui n’offrirait ni intérêt ni utilité.

Quant à l'expression terme d'exploitabitité employée pour exprimer l'âge de la coupe correspondant au but choisi, il est digne de remarque que :

Terme d'exploitabilité,

Age d'expluitation,

Révolution, 
sont absolument synonymes; nous avouons ne pas comprendre l'utilité de cette abondance dans les expressions; elle a le tort grave d'enlever la netteté aux idées; l'âge d'exploitation suffit au langage de l'économie forestière.

No 91. - Les problèmes sur l'âge d'exploitation sont les uns très faciles, les autres assez délicats à résoudre.

Chaque fois qu'il s'agit de trouver l'âge qui assure à l'exploitant une hauteur donnée ou une dimension capable de fournir une marchandise déterminée, la solution ne dépend que de l'observation des faits et de la végétation dans la localité. Cette solution concerne naturellement le produit principal et non les produits intermédiaires. Si, par exemple, on a calculé que pour produire des arbres de sciage, il faudra régler l'exploitation aux environs de 140 ans, il est certain que la forêt fournira en même temps des perches et des charpentes d'un âge moindre.

Pour les autres problèmes, par exemple pour ceux qui consistent à trouver l'âge qui assure la production la plus élevée ou le taux maximum du placement, il faut, considérer :

$1^{\circ}$ Que la solution varie avec chaque mode de traitement ;

$2^{\circ}$ Qu'on doit compter tous les produits principaux et intermédiaires.

C'est dans l'étude détaillée de chaque mode de traitement et non dans de simples éléments d'économie forestière, que ces problèmes peuvent être traités et résolus. Les solutions ne peuvent être générales; on se tromperait gravement en pensant, par exemple, que l'âge qui assure un taux de placement de $30 / 0$ pour une exploitation de peuplement d'un seul âge est le même que pour une exploitation de taillis sous futaie composée, installée en même sol et avec les mêmes essences. Nous nous borne- 
rons à donner ultérieurement quelques exemples de ces problèmes.

Une observation générale est nécessaire.

Les problèmes sur l'âge d'exploitation sont très utiles pour se rendre compte de ce que l'on fait et pour se décider à régler la coupe à un âge plutôt qu'à un autre. Mais c'est à une double condition :

La première est qu'on ne saurait exiger pour leur solution la précision mathématique. Si le calcul indique, par exemple, qu'à 25 ans on obtiendra un taux de placement déterminé, il faudra se dire que c'est dans les environs de cet âge que ce résultat sera atteint. Il y a, en effet, des variations dans les prix et dans les produits d'une même forêt qui ne permettent pas de préciser davantage : les limites d'exactitude dépendent de la nature des choses.

La seconde condition est de ne jamais généraliser et de se mettre en garde contre les conclusions absolues, de ne pas dire, par exemple, que telle exploitation convient à telle nature de propriétaires. Les conditions des propriétaires sont très variables; outre leurs différentes natures (communes, établissements publics, établissements d'utilité publique, particuliers, sociétés financières et autres, etc.), il y a dans chacune de ces classes des propriétaires plus ou moins riches, poursuivant des buts divers et ayant ainsi des objectifs différents pour l'exploitation d'une forêt. Telle famille qui a une grande fortune pourra très bien se contenter de ne retirer que 1 pour cent de ses forêts; tel qui demanderait 3 pour cent à son exploitation forestière pourra fort bien être satisfait de 2 pour cent s'il devient plus riche ou si le loyer général de l'argent vient à baisser. Il y a même de très modestes cultivateurs qui possèdent à côté de leur ferme quelques hectares de bois exploités à très long terme et que le taux de placement laisse très indifférents, soucieux qu'ils sont 
avant tout d'avoir sous la main les bois utiles aux réparations de leurs bâtiments.

Il ne faut donc pas généraliser et dire, par exemple, que les exploitations à court terme conviennent seules aux particuliers, parce que ce serait méconnaître la diversité de leurs situations. Il n'y a qu'un seul propriétaire, en France, susceptible d'une généralisation de cette nature, c'est l'État, parce qu'il est le pays tout entier. Pour lui, il n'y a qu'une exploitation convenable, c'est celle qui conduit les arbres jusqu'à leur maturité et ne les coupe qu'à l'âge le plus avancé où leur bois reste sain. Mais, est-il besoin de longs calculs et de conceptions bien savantes pour dire que l'État n'a à s'inquiéter ni du taux de placement, ni de celui de l'outillage, mais doit exploiter les forêts en vue des produits les plus utiles au travail national et qu'en fabriquant de gros et rieux arbres, il livrera, en général, au public les produits les plus utiles? La raison de ce maximum d'utilité n'est-elle pas que si d'un gros arbre on peut tirer de grosses et de petites pièces de bois, on n’a jamais pu demander une grosse pièce à un petit arbre?

En dehors de l'État, tous les autres propriétaires, communes, établissements publics, particuliers, etc., chercheront donc l'âge d'exploitation qui convient le mieux à leurs intérêts, et comme leurs intérêts sont très divers, il n'y aura jamais de règle systématique spéciale à une classe de propriétaires.

Mais que l'on soutienne qu'à raison des besoins de la vie, de la brièveté de l'existence et du morcellement des héritagres, les foréts des propriétaires particuliers sont en général exploitées à court terme, ceci est un fait dont la fréquence nous paraît malheureusement hors de doute ; mais c'est en même temps une question toute différente et que nous traiterons plus loin, en parlant du rôle économique des forêts de l'État. 
No 92. - Plus l'âge d'exploitation augmente, plus les produits de l'exploitation ont de valeur : on vendra plus cher 100 mètres cubes en bois d'arbres que 100 mètres cubes en bois de brins ou de perches.

Cette distinction entre l'arbre fait et les tiges inférieures correspond à la futaie.

On appelle futaie tout arbre dont le fût est formé. C'est une définition à la fois juridique et économique.

Dans la science du Droit, les arbres de futaie sont opposés aux taillis pour tracer les règles de l'usufruit (Code civil, art. 590 à 594) et pour établir une distinction entre les pouvoirs des officiers ministériels chargés des ventes publiques (lois du 22 pluviôse an VII et du 5 juin 1851).

Cette distinction est très ancienne, elle remonte au droit romain où la futaie s'appelait grandes arbores et le taillis sylva cadua; elle a passé dans notre Droit coutumier (Pothier, du Douaive $n^{\circ} 197$ ) et dans les ordonnances royales (ordonnance 1669, tit. 22, art. 5). Les coutumes déterminaient suivant les localités et d'une manière très variable, à quel âge un arbre devenait futaie ; aujourd'hui c'est une question de fait laissée à l'appréciation des tribunaux.

En économie forestière, la qualité d'arbre de futaie est déterminée par une circonstance facile à apprécier : la formation du fût. C'est le moment où l'arbre est fait et rend des services qu’on peut lui demander par la qualité de son bois : c'est à peu près la même définition qu'en droit.

La sylviculture n'adopte pas le même langage, elle distingue les forêts en deux régimes : celui du taillis et celui de la futaie. Dans le premier, la forêt se régénère par rejets de souches et, dans le deuxième, par les semences. Cette distinction, qui comporte un troisième régime mixte où la forêt se régénère à la fois par des rejets et par des semences (taillis sous futaie), est donc entièrement culturale; 
elle est étrangère à l'économie forestière et à la science juridique.

C'est le propre de chaque science d'employer les mêmes mots dans un sens tout différent. Il n'y a pas de définition absolue; les sylviculteurs feraient une erreur de droit en appelant futaie les jeunes recrus, les gaulis et les perchis venus de semences et dus au régime de la futaie. Les jurisconsultes se tromperaient également en croyant qu'il n'y a point de futaie dans les forêts soumises au régime du taillis.

No 93. - L'âge d'exploitation imprime un caractère spécial aux exploitations forestières. Que les exploitations soient d'arbres isolés ou de peuplements, qu'elles soient périodiques ou aménagées, il faut les distinguer en exploitations intensives et extensives.

L'exploitation intensive est celle qui se pratique à un âge avancé.

L'exploitation extensive se fait au contraire à des âges assez bas dont les taillis offrent l'exemple.

A cette distinction correspond une marque particulière, c'est que les exploitations intensives ont un gros capital d'exploitation et celles qui sont extensives un matériel beaucoup plus faible.

Il en est toujours ainsi pour les exploitations aménagées. L'intensité correspond à un grand matériel-bois immobilisé parce que le produit est annuel.

Voici, par exemple, 30 hectares de pineraie aménagée à 30 ans et produisant chaque année un liectare de coupe valant 900 francs, on évalue à 9,000 francs le matériel-bois de 1 à 29 ans, c'est une exploitation aménagée extensive ou à petit capital.

Les mêmes 30 heetares produiraient, si on les aménageait à 60 ans, une coupe de $0^{\mathrm{h}}, 50$ valant, à 3,600 fr. l'hectare, 1,800 francs par an. Le matériel de 1 à 59 ans vaiut $36,000 \mathrm{fr}$., c'est une exploitation intensive ou à gros capital. 
On voit que l'intensité de la culture forestière est, indépendante de l'étendue du sol et qu'un même terrain peut se prêter à des exploitations dont l'âge seul mesure l'importance de l'outillage et du produit.

Dans les exploitations périodiques intensives, le capitalbois est généralement faible; il se réduit à presque rien (souches et semis) dans les exploitations à blanc estoc; il est plus fort, mais toujours relativement peu considérable, dans les exploitations à réserves, mais le temps intervient pour aider à la fabrication du bois, et le temps n'est autre que de l'argent immobilisé faisant fonction de capital.

Un hectare de futaie pleine produit 7,000 francs tous les 120 ans. Le capital-bois est un semis naturel dont la valeur est de 100 sur un sol estimé 500 francs, mais il faut attendre le revenu pendant 120 ans; cette longue durée de l'épargne en fait une exploitation intensive.

Il peut être utile de connaître de quel capital-bois il faut doter une exploitation aménagée pour avoir, en revenu annuel, du bois d'un âge donné, 120 ans par exemple. Le taux de l'outillage d'une forêt aménagée est une question de relation entre le revenu et le matérielbois que nous traiterons $n^{\circ} 144$. Il ne faut pas la confondre avec celle du taux du placement qui est la relation entre le revenu et le capital engagé (sol et matériel d'exploitation). [No 122.]

Un mot encore sur cette distinction entre les exploitations à long et à court terme :

On a souvent dit qu'une exploitation est intensive quand ses peuplements sont bien complets et quand le sol est garni de tiges d'une manière aussi pleine que possible. Il y a, de ce côté, une confusion de mots contre laquelle il faut se mettre en garde: ce sont des exploitations complètes ou incomplètes et elles correspondent à un autre ordre d'idées que celles intensives et extensives. Pour celles-ci, l'économie forestière adopte la même définition 
que l'économie politique et l'agronomie où le mot est de langage courant : la culture intensive est celle qui affecte à l'exploitation un gros capital en bétail, en machines et en engrais, par opposition à l'agriculture extensive dont le capital est modeste; celle-ci est alor's forcée de s'étendre sur de vastes terrains dont elle tire peu de produits. De là, l'origine de son nom, mais la note caractéristique est l'augmentation du capital. Il en est absolument de même dans les exploitations forestières; elles ne sont intensives que par l'augmentation du capital-bois de l'exploitation ou, ce qui en est la conséquence, par l'élévation de l'âge fixé pour la coupe.

$\mathrm{N}^{\circ}$ 94. - Cette division entre les exploitations forestières à gros et à petit capital est donc analogue à ce qui existe en agriculture, où l'on distingue également la culture intensive et extensive. Mais une différence profonde doit être signalée :

Que les produits de l'agriculture viennent du champ de l'artisan ou de la grande culture d'une ferme bien outillée, ils se vendent au même prix et l'augmentation du capital d'exploitation ne peut avoir d'effet que pour accroître la quantité des produits agricoles. Il en est tout autrement dans les forêts : à mesure qu'on recule l'âge d'exploitation, le matériel immobilisé augmente, mais avec lui augmente, non la quantité, mais la qualité des produits.

La quantité : car si un hectare de 60 ans produit approximativement le double de bois qu'un hectare de 30 ans, la même contenance aménagée à 60 ans ne livrera, en revenu annuel, qu'une coupe de surface moitié moindre et la quantité des produits récoltés sera à peu près la même. La forêt est un bien naturel dans lequel aucun engrais, aucun amendement artificiel n'intervienment pour augmenter la production et il est fort probable que la forêt exploitée à 60 ans fixe autant de car- 
bone, c'est-à-dire fabrique autant de bois, à contenance égale, que la forêt exploitée à 30 ans.

La qualité, c'est-à-dire la valeur vénale des produits, augmente incontestablement avec l'âge. Tout le monde sait qu'un bois de 20 ans ne fournit que de la charbonnette à 4 francs le mètre cube, qu'à 25 ans environ on a du rondin dont le même volume et le même poids se vendent 8 francs. L'augmentation se continue pour les bois dont l'âge permet de fournir des pièces propres au service, puisque le mètre cube, c'est-à-dire la même unité de volume, peut se vendre jusqu'à 100 francs en chêne.

Quant au matériel-bois d'une même contenance, exploitée à court ou à long terme, il augmente à la fois de quantité et de qualité, c'est-à-dire de valeur vénale: de quantité, car chaque élément de contenance ayant travaillé plus longtemps à produire du bois, se trouve mieux garni de matière ligneuse; de qualité, car la suite d'âges qui constitue ce matériel étant plus âgée, a une valeur moyenne par mètre cube, plus considérable que dans l'exploitation à court terme.

L'exemple de 30 hectares de pineraie exploités à 30 ans et à 60 ans, déjà donné au no 93 , scrvira encole à mieux préciser le caractère de culture extensive et intensive des exploitations forestières.

On peut évaluer ainsi qu'il suit le matériel et le revenu de ces 30 hectares.

Exploitation de 30 hectares à 30 ans :

En volume $\{$ Matériel évalué à. ......... 2.250

$\left\{\right.$ Revenu : $1^{\mathrm{h}}$ à $150^{\mathrm{mc}} \ldots \ldots \ldots \ldots . .150$

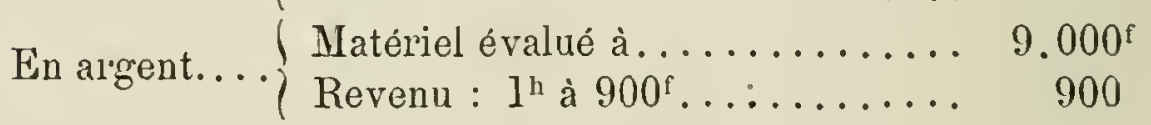

Exploitation de 30 bectares à 60 ans :

En volume... Matériel évalué à......... 4.500 mc

\{ Revenu : $0^{\mathrm{h}}, 50$ à $300^{\mathrm{mc}} \ldots \ldots \ldots . \quad 150$

En argent.... $\begin{aligned} & \text { Matériel évalué à. .......... } 36.000^{\mathrm{f}} \\ & \text { Revenu : } 0^{\mathrm{h}}, 50 \text { à } 3.600^{\mathrm{f}} \ldots \ldots \ldots \quad 1.800\end{aligned}$

On voit donc qu'en rendant son exploitation intensive, 
le propriétaire de forêt aura l'avantage, tout en fabriquant la même quantité de produits, de vendre ceux-ci plus cher; il aura surtout ce notable avantage d'augmenter à la fois son revenu et la valeur de son immeuble, d'être à la fois plus riche et mieux renté.

Reconnaissons, enfin, qu'il ne lui faudra pour y arriver ni grands frais d'imagination ni les soucis des industriels qui accroissent leur outillage; il lui suffira de reculer graduellement l'âge de sa coupe; il ne lui faudra que du temps et de l'épargne.

$\mathrm{N}^{\circ}$ 95. - Nous disons que, pour passer d'une exploitation extensive (ou à petit capital) à une exploitation intensive (ou à gros capital), il faudra du temps et de l'épargne. Il est facile de s'en rendre compte d'une manière très nette par l'exemple suivant:

Une commune possède 90 hectares de pineraie exploitée à 30 ans et produisant chaque année 3 hectares de coupe rendant 150 mètres cubes par hectare à $6 \mathrm{fr}$. l'un, soit $900 \mathrm{fr}$. l'hectare, ce qui fait 450 mètres cubes et $2,700 \mathrm{fr}$. pour la coupe annuelle.

On propose à cette commune d'aménager son bois à 90 ans, ce qui, dans l'avenir, ne changera pas sa production de 450 mètres cubes pour 1 hectare de coupe, mais lui fournira des produits bien plus précieux évalués à $16 \mathrm{fr}$. le mètre cube ou $7,200 \mathrm{fr}$. par an, au lieu de 2,700 fr.

On voit, immédiatement, par le diagramme de la figure 22, qu'il s'agit de passer d'un matériel d'exploitation formé de 1 à 30 ans à un autre matériel échelonné de 1 à 90 ans et qu'il faudra au moins 90 ans avant que le nouveau capital soit entièrement et régulièrement constitué.

On voit, ensuite, que ce nonveau matériel sera bien plus considérable en volume et en valeur que celui de l'exploitation aménagée de 1 à 30 ans; il faudra le former avec des économies faites sur la coupe annuelle, qui sera réduite à 1 hectare au lieu de 3 hectares.

$$
\begin{aligned}
& 1^{\text {ro }} \text { coupe }\left\{\begin{array}{l}
1^{\text {ro }} \text { année } 1^{\text {h }} \text { de } 30 \text { ans à } 150^{\text {mc }} \text { au lieu de } 450^{\mathrm{mc}}: 300^{\mathrm{mc}} \\
2^{\circ}-\mathrm{l} \text { de } 31 \text { ans à } 155
\end{array}\right. \\
& \text { fournira }\left(3^{\circ}-1 \text { de } 32 \text { ans à } 160 \quad-\quad 290\right.
\end{aligned}
$$




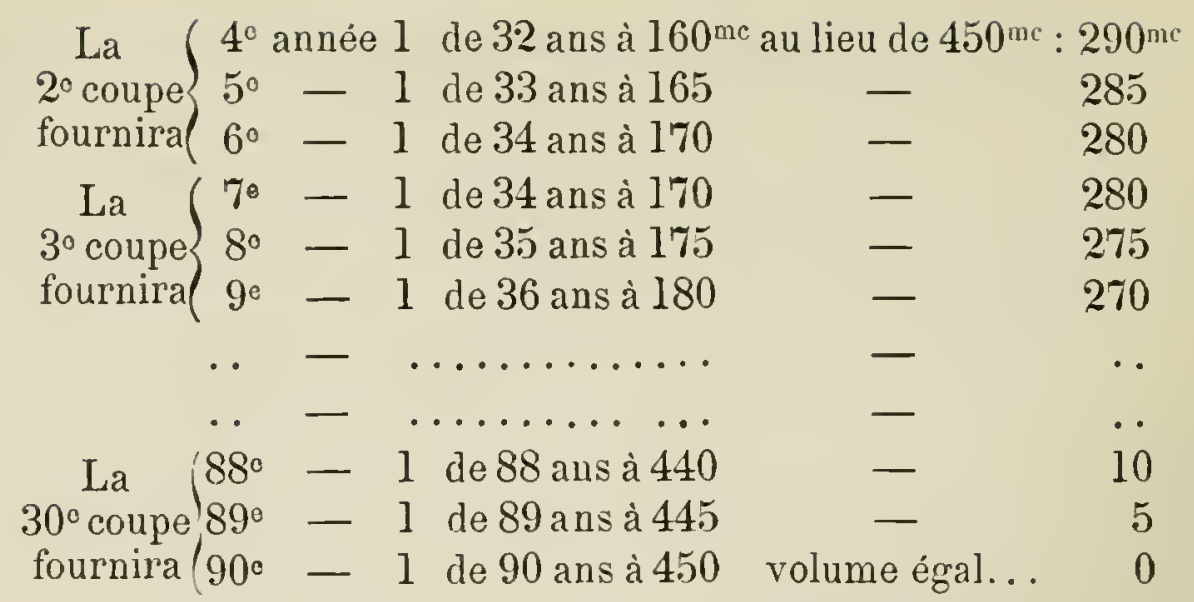

L'ẻpargne, très forte au début, va toujours en diminuant et en rendant le sacrifice moins lourd. Comme le prix du mètre cube passe de 6 à $16 \mathrm{fr}$., le moment arrivera assez vite ou le produit de l'exploitation, même réduit en volume, atteindra 2,700 fr., pour s'élever graduellement chaque année jusqu'à $7,200 \mathrm{fr}$., revenu présumé de l'exploitation établie à 90 ans.

Par des combinaisons plus ou moins ingénieuses, on arrive à atténuer la dureté des débuts dans une opération pareille, mais l'art de l'aménagiste ne pourra jamais échapper à cette loi fatale qui exige du temps et de l'épargne, pour passer d'une exploitation à court terme à une à long terme. Il pourra déplacer le moment du plus gros sacrifice, le reculer plus ou moins, le mettre au milieu et non au début de la période de transformation; il ne pourra pas plus l'atténuer que celui qui, voulant doubler les bâtiments d'une ferme, imaginerait des séries de combinaisons successives dans les locaux qu'il possède déjà.

Pour passer d'une exploitation extensive à une exploitation intensive, c'est-à-dire pour augmenter dans l'avenir sa fortune et son revenu, il faut donc du temps et de l'économie. Le temps est ce qui manque le moins aux propriétaires impérissables tels que les communes et l'Etat, mais si ces propriétaires veulent opérer cette utile transformation, en conservant le même budget et sans se résigner aux sacrifices nécessaires, ils sont à la recherche d'un problème insoluble et rêvent des utopies. 
$\mathrm{N}^{\circ}$ 96. - Rendre une exploitation plus intensive tout en conservant sa nature et son mode de traitement est une tout autre question que celle qui consiste à passer d'un mode de traitement à un autre et à modifier les éléments constitutifs de l'exploitation. On veut, par exemple, changer une expleitation d'arbres isolés (jardinage) en une exploitation de peuplements d'un seul âge (futaie pleine) parce qu'on croit celle-ci plus avantageuse. C'est, on le voit, une modification toute différente de celle qui consiste à augmenter l'âge du jardinage et à le rendre plus intensif.

La sylviculture appelle conversion le changement d'un régime en un autre et transformation le passage d'un mode de traitement à un autre dans le même régime. La sylviculture n'envisage que le moyen de régénération par les souches ou par la semence (taillis et futaie) et le mode cultural dans le même régime. On convertit, par exemple, un taillis en futaie; on transforme un taillis ordinaire en taillis fureté, une futaie jardinée en futaie pleine. Le plus souvent on cherche à allonger l'âge d'exploitation, mais cet ordre d'idées n'entraine pas forcément et par lui seul, le passage d'une exploitation à petit capital à une exploitation à gros matériel. Ainsi la transformation d'une futaie jardinée en futaie pleine pourra très bien s'effectuer en conservant le mème âge d'exploitation et le même matériel; la forèt sera autrement cultivée et le matériel autrement disposé, mais voilà tout. Ainsi, encore, un taillis aménagé à 30 ans pourra fort bien être converti en pineraie exploitée au mème âge et qui, se régénérant par la semence, sera de la futaie dans le sens cultural du mot sans l'être dans le sens juridique et èconomique.

La distinction que nous faisons entre les exploitations intensives et extensives à long et à court terme, à gros et à petit capital est donc uniquement économique. Elle existe aussi bien dans les forêts aménagées que dans les exploitations périodiques.

Le changement économique d'une exploitation extensive en une exploitation intensive ne porte que sur l'âge 
d'exploitation en conservant le même mode de traitement cultural. Les conversions culturales de taillis sous futaie en forêts de peuplements pleins, dits futaies, modifient à la fois l'âge d'exploitation, le régime et le traitement de la forêt; elles ont pour but de créer des arbres de futaie dans un avenir éloigné; elles sont naturellement impuissantes à les créer immédiatement. Le sylviculteur qui s'est occupé depuis 30 ans à substituer aux taillis des jeunes fourrés de semis naturels n'a pas créé de futaie dans le sens utile et profitable du mot. Si pour y arriver il a sacrifié de beaux et bons arbres de futaie existant dans la forêt, il a commis un acte anti-économique. Celui qui, au contraire, réservant à chaque coupe un certain nombre des plus beaux brins qui poussent dans ses taillis, en a fait des arbres, en grand nombre, et sans gros sacrifice immédiat, a réellement fait des futaies; il a enrichi lui-même et son pays! 


\section{PRODUIT DE L'EXPLOITATION}

Article $1{ }^{\mathrm{cr}}$.

\section{Produit en matière.}

$\S 1$ er. - Production.

Nos 97. - Définition et mesure de la production.

98. - Calcul de la production annuelle.

99. - Calcul de la production moyenne.

100. - Observations sur la production moyenne.

No 97. - La quantité dont une forêt s'accroît annuellement forme la production annuelle. Pour les études de physiologie et de chimie appliquées aux forêts, on compte dans la production tout ce que le sol fournit : bois, racines, feuilles, graines, etc... Cette production se mesure par le poids des matières desséchées et dont on indique le degré de dessiccation.

Mais l'économie forestière n'envisageant que la fabrication du bois, on ne compte dans la production que le bois des tiges et des branches, en négligeant même les racines; on la mesure par le volume exprimé en mètres cubes supposés pleins et sans interstice.

On distingue la production annuelle et la production moyenne: la première est le volume dont la forêt s'accroît par an, la seconde est le volume dont elle s'accroît pendant un certain temps, divisé par le nombre d'années. 
La production annuelle et la production moyenne se rapportent aussi à la contenance en les divisant par la surface pour avoir la production par hectare. Il y a, sur ce point, une habitude dont il faut tenir compte: c'est que la production des peuplements est généralement rapportée à l'hectare, tandis que celle des arbres isolés ne l'est pas par la raison qu'on ne connaît pas la surface occupée par eux. C'est pour aroir méconnu cette habitude que la confusion s'est souvent introduite dans les idées des forestiers.

Exemple. - 10 hectares de sapiniere de 100 ans ont un volume de 7,930 mètres cubes; on constate que de 99 à 100 ans la forêt a gagné 105 mètres cubes.

La production annuelle à 100 ans est....... 105

Par hectare.............. 10,500

La production moyenne est de $\frac{7930}{100}=\ldots \ldots . \quad 79,300$

Par hectare............... 7,930

Un sapin de 130 ans cube....... $2^{\mathrm{mc}}, 650$

Sa production de 129 à 130 ans est de 0,055

Sa production moyenne est de $\frac{2,650}{130}=\quad 0,0204$

Les forestiers ont souvent donné le nom de feuille à la production annuelle pour mieux marquer que celle-ci est le résultat de la frondaison de chaque année: Production annuelle, feuille et accroissement annuel sont donc des expressions synonymes. La production moyenne est l'accroissement moyen pendant le nombre d'années qu'on considère.

Enfin, on a souvent rapporté la production annuelle ou moyenne non plus à la contenance, mais au volume acquis: on dit ainsi qu'un arbre ou un peuplement rapporte en un an $20 / 0$ de son volume. Il est important de ne pas confondre ces divers modes de mesure.

$\mathrm{N}^{\circ}$ 98. - La production annuelle est fort difficile à me- 
surer parce que chaque année le produit s'ajoute au bois acquis et l'on ne connaît pas encore la loi de formation de la matière ligneuse qui n'est point, dès lors, exprimée par une relation mathématique. La manière de calculer cette production annuelle est donc approximative et nous allons indiquer les procédés employés.

$1^{\circ}$ On peut cuber le volume de l'arbre ou du peuplement à des époques connues, tous les dix ans, par exemple, et diviser la différence de volume par le nombre d'années ;

Par exemple, un chêne de 150 ans cube..... $2^{\text {mc }}, 380$ 10 ans après, il a un volume de.......... 2,700 il a acquis en 10 ans .............. Soit par année $\frac{0,320}{10}=0^{\mathrm{mc}}, 032$ ou 32 litres.

Un peuplement de sapin cubait à 100 ans.... $793^{\mathrm{mc}}$ il cube à 110 ans................... 900

il a acquis en 10 ans $\ldots \ldots \ldots \ldots \ldots \ldots \ldots . \ldots \ldots 7^{\mathrm{mc}}$ soit par an ..................... 10,700

Cette manière de mesurer la production annuelle ne fournit en réalité que la production moyenne pendant une période d'années; elle n'est donc qu'une mesure approximative, qui a, de plus, l'inconvénient de n'être pas toujours praticable à raison du temps pendant lequel on doit observer la forêt;

$2^{\circ}$ On a cherché un moyen de mesurer approximativement la production annuelle d'après le volume acquis au moment que l'on considère.

A) Voici un procédé pour l'arbre isolé : la production annuelle u à l'âge $n$ est en fonction du volume.acquis $\mathrm{V}$ :

$$
v=\mathrm{V} \times \frac{1}{1 / 2^{n}}
$$

Pour obtenir la production annuelle d'un arbre, it suffit de multiplier son volume par l'inverse de la moitié de son âge. 
Un chêne de 120 ans cubant $1^{\mathrm{mc}}, 430$ produit pour la cent vingtième année

$$
\frac{1,430}{60}=0^{\mathrm{mc}}, 024=1,660 / 0
$$

Démonstration: Le volume d'un arbre peut se mettre sous la forme:

$$
\mathrm{V}=f \mathrm{H} \mathrm{D}^{2}
$$

dans laquelle $f$ est un facteur de forme, $H$ la hauteur totale, $\mathrm{D}$ le diamètre mesuré à $\mathrm{1}^{\mathrm{m}}, 30 \mathrm{du}$ sol.

Si on cherche l'augmentation de volume $x$ résultant d'une faible augmentation de diamètre $\delta$, on peut admettre que pour cette faible augmentation, la liauteur $\mathrm{H}$ et la forme $f$ ne changent pas (fig. 2/4):

$$
\begin{gathered}
\mathrm{V}=\mathrm{KD} \mathrm{D}^{2} \\
\mathrm{~V}+x=\mathrm{K}(\mathrm{D}+\delta)^{2}=\mathrm{KD} \mathrm{D}^{2}+2 \mathrm{KD} \delta+\mathrm{K}^{2} \\
\text { d'où } x=2 \mathrm{~K} \delta+\mathrm{K}^{2} \\
\frac{x}{\mathrm{~V}}=\frac{2 \delta}{\mathrm{D}}+\frac{\delta^{2}}{\mathrm{D}^{2}}
\end{gathered}
$$

négligeant le second terme qui est très faible si ò est petit par rapport à $\mathrm{D}$, on a :

et si $\delta=1$ on a

$$
\frac{x}{\mathrm{~V}}=\frac{\delta}{1 / 2 \mathrm{D}} \text { et } x=\mathrm{V} \times \frac{\delta}{1 / 2 \mathrm{D}}
$$

$$
x=\mathrm{V} \times \frac{1}{1 / 2 \mathrm{D}}
$$

Pour obtenir l'augmentation de volume due à un accroissement d'un centimètre sur le diamètre, il suffit de multiplier le volume par l'inverse du demi diamètre exprimé en centimètres ( $\left.{ }^{1}\right)$.

Or, si on désigne par $\alpha$ l'épaisseur de la couche annuelle moyenne formée sur la souche, $\mathrm{D}=\alpha$ n, et si on admet que l'épaisseur de l'année $n$ est égale à la moyenne des années antérieures, $\delta=\alpha$, la formule devient

$$
x=\mathrm{V} \frac{1}{1 / 2^{n}}
$$

( $\left.{ }^{1}\right)$ La mème formule existe naturellement pour la circonlérence : $x=\mathrm{V} \times \frac{1}{1 / 2 \mathrm{C}}$. 
La production anmuelle d'un arbre s'obtient en multipliant son volume par l'inverse de la moitié de son âge.

Un chène de 120 ans cubant $1^{\mathrm{mc}}, 430$ s'accroît de $\frac{1}{60}=0^{\mathrm{mc}}, 024$ Un chêne de 150 ans cubant $2^{\mathrm{mc}}, 380$ s'accroit de $\frac{1}{75}=0^{\mathrm{mc}}, 032$

Ce procédé fournit une expression un peu faible de la production annuelle. Il suppose que la couche d'une année a une épaisseur égale à celle de la moyenne des années antérieures. Cette hypothèse est admissible pour un calcul approximatif, mais il peut fort bien arriver que, les couches annuelles diminuant d'épaisseur, celle de l'année considérée soit moindre que l'épaisseur moyenne des années antérieures. Dans ce cas, on mesure directement l'épaisseur ¿ de la couche annuelle en millimetres pour appliquer la formule $\mathrm{V} \times \frac{\delta}{1 / 2 \mathrm{D}}$. On a même inventé dans ce but desinstruments perforateurs pour extraire un petit cylindre de l'arbre (Zuwachsborer de II. Pressler).

On peut se rendre compte de la production annuelle d'un arbre isolé par le tableau suivant :

A 20 ans $\frac{1}{10}$ ou $100 / 0$

A 30 ans $\frac{1}{15}$ ou $6,660 / 0$

A 40 ans $\frac{1}{20}$ ou $50 / 0$

A 50 ans $\frac{1}{25}$ ou $40 / 0$

A 60 ans $\frac{1}{30}$ ou $30 / 0$

A 80 ans $\frac{1}{40}$ ou $2,500 / 0$

A 100 ans $\frac{1}{60}$ ou $1,660 / 0$

A 150 ans $\frac{1}{75}$ ou $1,330 / 0$

A 200 ans $\frac{1}{100}$ ou $10 / 0$

Il ne faut pas oublier que ce tableau suppose que l'épaisseur de la couche ligneuse de l'année est égale à la moyeune des épaisseurs des années antérieures. 
B) Quant au peuplement d'un seul âge, le calcul de la production annuelle est encore plus incertain, parce qu'on ne connaît pas la loi de formation du volume par ces associations d'arbres.

On peut cependant admettre que la production à l'âge $n$ sera exprimée en fonction du volume acquis $\mathrm{V}$ par

$$
v=\mathrm{V} \times \frac{1}{n}
$$

La production d'un peuplement de 100 ans cubant $793^{\mathrm{mc}}$ serait à 100 ans de

$$
\frac{793}{100}=7^{\mathrm{mc}}, 930
$$

Ce procédé suppose que la production annuelle est égale à la production moyenne, en d'autres termes, que la quantité de bois fabriqué annuellement est une quantité constante. La surface foliacée d'un peuplement formé de tiges d'un seul âge offrant une quantité constante, on en déduit que la fixation du carbone s'opère chaque année de la même manière pour fabriquer une quantité égale de matière ligneuse. Cette hypothèse n'est point entièrement exacte. Sans doute le développement foliacé varie peu pendant un certain nombre d'années pour un peuplement bien complet; mais il arrive un moment où les tiges et les branches s'éclaircissent, les feuilles diminuent en nombre et la quantité de bois fabriqué va en décroissant chaque année. Le calcul précédent n'est donc qu'une approximation, qui suffit aux évaluations habituelles, mais qui peut ne pas représenter l'expression réelle de la production annuelie à un moment donné.

Le calcul de la production annuelle dans les peuplements à coupes d'éclaircie est encore plus difficile. La production comprend, en effet, l'accroissement du peuplement et, en outre, celui de la coupe d'éclaircie.

Or, on ne connaît la loi de formation"ni du produit prin- 
cipal, ni du produit intermédiaire et on ne peut mesurer la production annuelle que par analogie avec la production moyenne.

C) Quant aux forêts mixtes formées d'arbres et de peuplements, il faut évaluer la production annuelle séparément pour les arbres et pour le peuplement. Cela peut se pratiquer pour les taillis sous futaie où les arbres sont isolés par l'action de l'homme, mais dans les forêts jardinées ou furetées, exploitations d'arbres isolés par la seule sélection naturelle, il faut s'en tenir au premier procédé qui a été indiqué, c'est-à-dire à l'observation de la forêt pendant un certain temps, cinq ou six ans, par exemple. On mesure les tiges à la hauteur usuelle, $\mathrm{I}^{\mathrm{m}} 30$, en marquant cette hauteur par un trait circulaire en couleur afin que les mesurages ultérieurs soient effectués dans les mêmes conditions.

No 99. - Il est beaucoup plus facile de calculer la production moyenne de l'arbre et du peuplement d'un seul âge : il suffit de diviser le volume par l'âge pendant lequel il a été acquis.

$1^{0} 50$ hectares de pins âgés de 50 ans fournissent $12,500 \mathrm{mè-}$ tres cubes.

$$
\begin{gathered}
\text { Production moyenne } \frac{12500}{50^{\text {ans }}}=250^{\mathrm{mc}} \\
\text { Par hectare } \frac{250}{50^{\mathrm{hect}}}=5^{\mathrm{mc}}
\end{gathered}
$$

$2^{\circ}$ Un chêne de 120 ans cube $1^{\mathrm{mc}}, 430$.

$$
\text { Production moyenne } \frac{1,430}{120^{\text {ans }}}=0^{\mathrm{mc}}, 012
$$

Quant à la production par hectare, il faudrait connaitre la surface occupée par l'arbro de 120 ans pour rapporter cette production moyenne à l'unité de contenance.

Il ne faut pas comprendre la production moyenne d'un massif forestier avec la production moyenne d'une exploitation; celle-ci se calcule non sur le temps que les pro- 
duits ont mis à naître, mais sur la durée totale de l'exploitation. Dans les exploitations simples, ces deux durées se confondent, mais elles se distinguent dans les exploitations composées, comme on le voit dans l'exemple suivant:

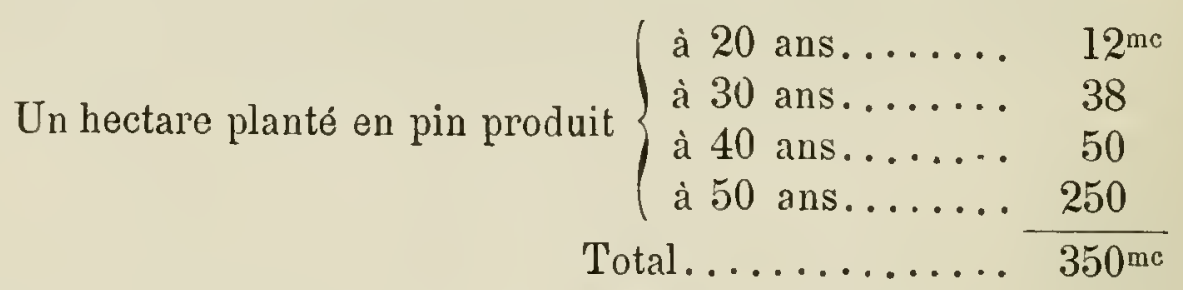

La production moyenne est de $\frac{350}{50^{\text {ans }}}=7^{\mathrm{mc}}$

Le calcul suivant donnerait la production moyenne non de l'exploitation, mais du peuplement.

$12^{\text {me }}$ accuis en 20 ans, production $0^{\text {me }}, 600$

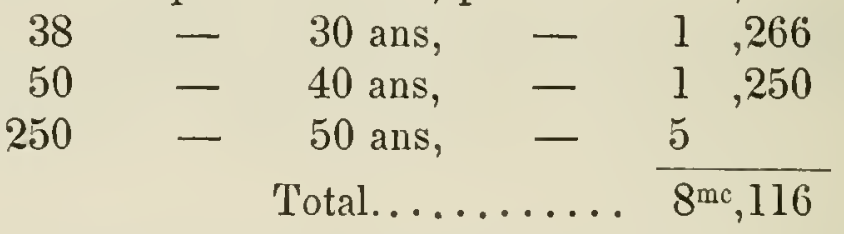

On conçoit, en effet, que pendant les 50 ans qui forment la durée de l'exploitation, les produits recueillis à diverses époques se répartissent sur toute la durée de l'exploitation; celle-ci seule doit être considérée quand on veut calculer ce que la forêt a produit par année moyenne.

Voici, pour autre exemple, un hectare de taillis sous futaie, âgé de 30 ans et présentant 98 arbres. Les arbres ayant été acquis en 30,60 et 90 ans, la production moyenne de ces arbres et du taillis est de $5^{\mathrm{mc}}, 589$, savoir :

Pour le taillis cubant ...... $150^{\mathrm{mc}}$, product. $\frac{150}{30}=5^{\mathrm{mc}}$

Pour 60 modernes de 60 ans cubant à $0,300 \ldots \ldots \ldots .18 \quad-\frac{18}{60} \quad 0 \quad 300$

Pour 30 anciens de 90 ans cubant à $0,700 \ldots \ldots \ldots 21$

$-\quad \frac{21}{90} \quad 0 \quad 223$

Pour 8 bis-anciens de 120 ans cubant à $1,375 \ldots \ldots \ldots$.

$11-\frac{11}{120} \quad 0,066$

Total ............ $\overline{5^{\mathrm{mc}}, 589}$ 
La production moyenne de l'exploitation est, au contraire, de $6^{\mathrm{mc}}, 180$.

On obtient, en effet, chaque 30 ans, un revenu de:

$$
\begin{aligned}
& 30 \text { modernes eubant } 0^{\mathrm{mc}}, 300 \ldots .9^{\mathrm{mc}} \text { » } \\
& 22 \text { anciens } \quad-\quad 0,700 \ldots \ldots .15,400 \\
& 8 \text { bis-anciens }-1,375 \ldots .11 \text { » } \\
& 35^{\mathrm{mc}}, 400 \\
& \text { Taillis de } 30 \text { ans............. } 150 \text { " } \\
& \text { Total ........ } 185^{\mathrm{mc}}, 400
\end{aligned}
$$

ce qui donne pour production moyenne de l'exploitation :

$$
\frac{185,400}{30}=6^{\mathrm{mc}}, 180
$$

C'est pour avoir confondu ces deux manières d'envisager la production moyenne que certaines personnes ont critiqué les méthodes actuelles d'exploitation et ont prétendu qu'elles faisaient rendre au propriétaire tantôt moins, tantôt plus que la production acquise, ce qui conduisait à chercher de nouveaux procédés pour établir un rendement plus rationnel.

$\mathrm{N}^{\circ}$ 100. - Le calcul de la production moyenne étant ainsi très simple, on a pu se rendre compte de la manière dont l'arbre et le peuplement produisent la matière ligneuse, en moyenne par année.

Voici, pour exemple, des chênes dont le volume a été relevé de 30 en 30 ans dans une forêt de taillis sous futaie régulièrement exploitée et a été constaté par une expertise faite en 1875 dans la forêt de Merlanvaux (Belgique) :

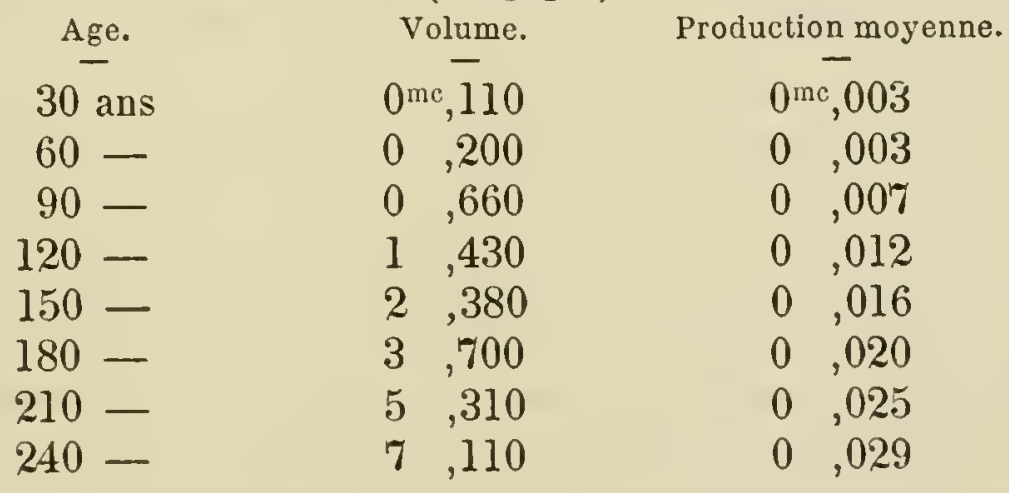


Voici également une sapinière dont les volumes sont empruntés à M. le professeur Lorey; par hectare :

\begin{tabular}{|c|c|c|c|}
\hline Age. & Volume. & \multicolumn{2}{|c|}{ Production moyenne. } \\
\hline 20 ans & $47^{\mathrm{mc}}$ & & c,300 \\
\hline $30-$ & 92 & 3 & 100 \\
\hline $40-$ & 158 & 3 &, 900 \\
\hline $50-$ & 240 & 4 & ,800 \\
\hline $60-$ & 333 & 5 & , 500 \\
\hline $70-$ & 436 & 6 & , 200 \\
\hline $80-$ & 547 & 6 & ,800 \\
\hline $90-$ & 673 & 7 &, 500 \\
\hline $100-$ & 793 & 7 &, 900 \\
\hline $110-$ & 900 & 8 &, 100 \\
\hline $120-$ & 985 & 8 & ,200 \\
\hline $130-$ & 1055 & 8 & 100 \\
\hline $140-$ & 1105 & 7 & 900 \\
\hline
\end{tabular}

On voit, par ce double exemple, que la production moyenne de l'arbre va toujours en augmentant, ce qui est naturel, puisque l'arbre occupe un terrain de plus en plus grand.

Au contraire, la production moyenne du peuplement débute faiblement, grandit successivement et passe par un maximum (à 120 ans dans l'exemple ci-dessus) à partir duquel la production diminue. Le peuplement est, en effet, enfermé dans une surface limitée et constante, la surface foliacée doit avoir un développement de plus en plus grand ou une activité de plus en plus féconde jusqu'au moment où l'éclaircissement se produit et la production diminue. C'est du moins ce qui paraît résulter des tables publiées en Allemagne par différents auteurs, depuis Cotta jusqu'aux directeur's des stations d'expériences.

La différence qu'on remarque dans la marche de la production moyenne des arbres et des peuplements est plus apparente que réelle, car si on calculait la surface occupée par l'arbre à ses différents âges et si on rapportait la production moyenne à l'unité de contenance, on remarquerait, sans doute, la même loi accroissante et 
ensuite décroissante que pour les peuplements. Mais ce calcul des surfaces occupées par l'arbre à ces différents âges n'a point encore été effectué et nous ne possédons aucune donnée expérimentale sur cette intéressante question.

Si, d'un autre côté, on réunissait aux produits principaux de la sapinière, les produits intermédiaires dus aux éclaircies faites à différents âges, on constaterait peut-être que la production moyenne tend à devenir constante ou à reculer le moment du maximum : ici encore, la donnée expérimentale manque.

\section{§2. - Revenu.}

Nos 101. - Détinition.

102. - Revenu annuel et production par année moyenne.

103. - Remarque sur la production comparée au revenu.

104. - Recherche de l'âge d'exploitation donnant la plus grande production en matière; exploitation de peuplements.

103ั. - Nême question pour l'arbre isolé.

$\mathrm{N}^{\circ}$ 101. - La définition du droit romain : quidquid de re nasci et renasci potest, donne très bien l'idée du revenu : tout ce qui naît et renaît de la chose, d'une manière constante et soutenue.

On distingue ainsi le revenu périodique et le revenu annuel.

Le revenu est toujours égal à la production; il en résulte que le revenu d'une exploitation périodique à 25 ans, par exemple, est formé par les 25 productions annuelles qui se sont accumulées pour former l'arbre ou le peuplement de 25 ans. Il est égal, ainsi, à 25 productions par année moyenne.

Dans une exploitation aménagée, le revenu annuel est égal à la production annuelle acquise sur le matériel-bois qui garnit la forêt. Ce matériel reste constant précisément parce qu'on lui enlève chaque année la quantité dont il 
s'est accru. On concoit qu'il n'est pas facile de recueillir sur toute l'étendue cette quantité dont la forêt s'est augmentée chaque année. Or, comme la forêt aménagée à 25 ans, par exemple, est formée de 25 arbres ou de 25 contenances égales âgées de 1 à 25 ans, c'est-à-dire de 25 exploitations périodiques dont chacune se fait à 25 ans successivement chaque année, on voit que la coupe de l'arbre ou du peuplement de 25 ans fornera le revenu annuel et que ce revenu fournira bien la production annuelle des 25 arbres ou, en d'autres termes, 25 fois la production moyenne d'un arbre de 25 ans.

La figure 23 montre par les productions annuelles teintées en noir, que la coupe de 25 ans exprime tout l'accroissement annuel de la forêt et qu'elle représente 25 fois la production moyenne du vingt-cinquième de la contenance ou, enfin, 25 fois la production moyenne de l'unité de contenance.

$\mathrm{N}^{\circ}$ 102. - Si on compare l'exploitation périodique et l'exploitation aménagée au même âge, on constate que, pour une même contenance, le revenu annuel est égal à la production par année moyenne. Le revenu annuel par hectare est égal à la production moyenne par hectare et par an. Une forêt aménagée fournit chaque année 5 mètres cubes par hectare; une forêt de même contenance et de mêmes essences, en même terrain, mais exploitée périodiquement au même âge, rapportera 5 mètres cubes par année moyenne.

Il suffit pour s'en convaincre d'examiner la figure 23, elle montre que si $\mathrm{R}$ désigne le revenu annuel et $\mathrm{P}$ la production totale de la contenance $\mathrm{S}$ exploitée à $n$ ans

$$
\frac{\mathrm{R}}{\mathrm{S}}=\frac{\mathrm{P}}{n \mathrm{~S}}
$$

et, par suite :

$$
\mathrm{P}=\mathrm{R} n
$$


ainsi l'exploitation aménagée fournit chaque année ce que l'exploitation périodique rend par hectare et par année moyenne et son revenu annuel est égal à la production moyenne de la surface.

Il en résulte que, dans les recherches de statistique, le revenu d'un ensemble de forêts peut être exacteinent considéré comme la production moyenne de ces forêts et qu'il suffit d'examiner les revenus à différentes époques pour comparer les productions moyennes.

La même remarque est applicable aux exploitations d'arbres isolés, mais à la condition de rapporter à l'hectare leur production moyenne. Or, il est digne de remarque qu'une suite d'arbres aménagée à $n$ ans occupe à un moment quelconque, une surface constante, mais que chaque arbre exige individuellement une surface égale à celle qu'il occupera à l'âge de coupe. Il en résulte que le terrain nécessaire à une exploitation aménagée à $n$ ans est égal à $n$ fois le couvert de l'arbre de coupe, tandis que le sol réellement occupé est beaucoup moindre. Or, dans les exploitations d'arbres faites sur les taillis ou dans les forêts jardinées, on ne mesure pas la place nécessaire aux arbres ; celle-ci leur est toujour's assurée et on évite l'inconvénient de calculer leur couvert, en réglant le revenu par pieds d'arbres.

Alors, quand on dit que le revenu annuel est égal à la production moyenne, il faut ne pas oublier qu'il s'agit de la production des arbres et non de la production par hectare, et que la règle n'est exacte pour eux qu'à la condition de faire abstraction de la contenance qu'ils occupent et qu'on leur suppose toujours largement assurée dans la surface affectée à l'exploitation.

Pour éviter les confusions, on a pensé à réserver le mot production pour les cas où on l'envisage par rapport au sol et celui d'accroissement pour l'arbre, abstraction faite de la surface qu'il occupe. Cette distinction est bien 
subtile et peu pratique. Il suffit de distinguer la production du sol de la production de l'arbre, dans les cas où il y a lieu de parler de l'une et de l'autre. Il suffit, surtout, de ne jamais méconnaître le caractère des exploitations d'arbres isolés et de ne pas régler leur revenu par le volume calculé sur la contenance: le revenu des futaies sur taillis et des forêts jardinées doit toujours être exprimé par pieds d'arbres.

$\mathrm{N}^{\circ}$ 103. - La règle précédente n'est exacte qu'à la condition expresse que la production moyenne sera mesurée sur la durée de l'exploitation et non sur le temps pendant lequel les produits ont été acquis, comme nous l'avons fait remarquer $n^{\circ} 99$.

Reportons-nous à la pineraie exploitée à 50 ans, prise pour exemple $\left(\mathbf{n}^{\circ} 99\right)$; une exploitation de 50 hectares aménagée à 50 ans fournira un revenu annuel de 350 mètres cubes.

Coupe principale à 50 ans........ $250^{\mathrm{mc}}$

Coupe d'éclaircie à 40 ans........ 50

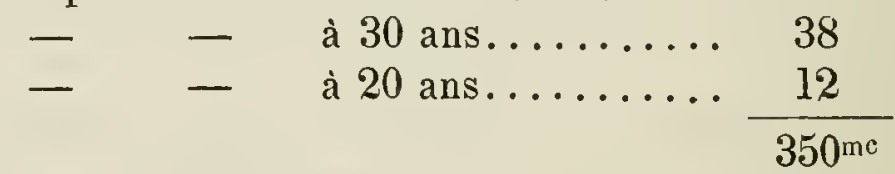

Le revenu annuel par hectare est :

$$
\frac{350}{50^{\text {hoct }}}=7 \mathrm{mc}
$$

$\mathrm{Si}$ on avait calculé la production moyenne sur le temps pendant lequel les produits ont "été acquis, on aurait obtenu $8^{\mathrm{mc}}, 116$ par année moyenne ( $\left.\mathrm{n}^{\circ} 99\right)$ et l'aménagement aurait eu pour effet de produire moins chaque année que l'exploitation périodique par année moyenne, ce qui est impossible.

De même 30 hectares de taillis sous futaie aménagés à 30 ans avec un plan de balivage de 60 baliveaux, 30 modernes et 8 anciens, fournira un revenu annuel de $185^{\mathrm{me}}, 400$.

Taillis ................. $150^{\mathrm{mc}}$ "

30 modernes à $0,300 \ldots \ldots \ldots \ldots .9 . \quad 9$,

22 anciens à $0,700 \ldots \ldots \ldots \ldots \ldots, 15,400$

8 bis-anciens à $1,375 \ldots \ldots \ldots \ldots \frac{11 \quad \text { " }}{185,400}$

$185^{\mathrm{mc}}, 400$ 
Le revenu par hectare et par an est $6^{\mathrm{mc}}, 180$.

$$
\frac{185,4}{30^{\text {hoct }}}=6^{\mathrm{mc}}, 180
$$

Le calcul de la production, effectué sur le temps pendant lequel les arbres ont été acquis, fournit une production par année moyenne de $5^{\mathrm{mc}}, 589$ et l'aménagement aurait pour effet de produire ici plus que la production moyenne de l'exploitation périodique.

On comprend que des résultats aussi disparates ne proviennent que de la manière dont la production moyenne a été calculée : la pineraie aménag'ée fournit un revenu de 7 mètres cubes par hectare parce que la durée de la réalisation est plus grande que celle de la formation des produits; les éclaircies ont été acquises en 20,30 et 40 ans, mais n'ont été perçues que pendant 50 ans. De même, dans la futaie sur taillis, les arbres ont été acquis en 60,90 et 120 ans. Or dans l'espace de 30 ans, on a réalisé un certain nombre de ces arbres, en laissant les autres sur pied : la période de réalisation étant plus courte que le temps de formation, la production moyenne est plus considérable.

Bien des erreurs ont été commises par cette confusion dans la manière de mesurer la production moyenne. Des procédés d'estimation ont été fondés sur la réalisation de tous les arbres dans le laps de temps où ils ont été produits, et des recherches fantaisistes ont été entreprises pour prétendre qu'on pouvait faire rendre davantage aux forêts.

$\mathrm{N}^{\circ}$ 104. - La remarque faite au $\mathrm{n}^{0} 102$ va nous servir à résoudre dans la limite du possible le problème qui consiste à chercher à quel âge il faut régler une exploitation pour que le revenu annuel fournisse la plus grande quantité de matière ligneuse possible.

Nous avons vu que l'exploitation périodique fournit par hectare et par année moyenne la même production que 
celle fournie chaque année par l'exploitation aménagée. Il en résulte qu'il suffira de raisonner sur le revenu de l'exploitation périodique pour que la solution soit applicable à l'exploitation aménagée.

Le revenu étant égal à la production moyenne ( $\left.\mathrm{n}^{\circ} 102\right)$, on obtiendra la plus grande quantité de matière ligneuse en réglant l'exploitation à l'âge qui correspond à la production moyenne maxima. Il s'agit donc de trouver cette époque du maximum dans la production moyenne: nous examinerons à cet effet le peuplement et l'arbre.

$1^{\circ}$ Peuplement. - Dans l'état actuel de nos connaissances, le maximum de production moyenne d'un peuplement d'un seul âge ne peut se révéler que par l'observation du peuplement à ses divers âges, soit par la comparaison directe sur des sols et des essences de même nature, soit par des tables de production comme il en a été publié en Allemagne.

Le quotient de la division du volume à l'hectare par l'âge donnant la production moyenne, on voit que dans la sapinière du $n^{\circ} 100$ l'âge de 120 ans fournira un revenu annuel de $8^{\text {mec }}, 200$ par hectare, tandis que si on fixait la coupe à tout autre âge, on aurait un revenu inférieur; il en est de même pour l'exploitation périodique : réglée à 120 ans, celle-ci fournira en 120 ans

$$
8,200 \times 120=984^{\mathrm{mc}}
$$

réglée à 60 ans, elle fournira 2 coupes qui, en 120 ans, ne donneront que 660 mètres cubes.

$$
5,500 \times 60+5,500 \times 60=660
$$

Mais à quel moment de la vie d'un peuplement devra-ton rencontrer ce maximum de production moyenne? On a dit qu'il arrivait à l'âge où les arbres out atteint la pleine fertilité. D'autres ont soutenu qu'il se rencontrait à l'époque où le fût est constitué et où son allongement en hauteur cesse de s'accroître. Rien ne le prouve, car les 
lois de la végétation n'ont pas encore été déterminées par l'expérience!

On a dit aussi que le maximum du rendement en matière arrivait quand la production annuelle était égale à la production moyenne. Ceci est une vérité certaine, car, lorsque la feuille (production annuelle) n'ajoute au volume qu'une quantité égale à la production moyenne antérieure, $\frac{\mathrm{V}}{n}$, celle-ci ne peut plus augmenter. Le quotient de la division du volume ainsi accru, $V+\frac{V}{n}$ par l'âge suivant $n+1$, ne peut plus, alors, être qu'égal au précédent quotient $\frac{\mathrm{V}}{n}$. En effet

$$
\mathrm{V}+\frac{\mathrm{V}}{n}: n+1=\frac{\mathrm{V}}{n}
$$

Mais c'est une vérité bien inutile pour guider l'exploitant dans la recherche de l'âge qui lui fournira la production maxima, puisqu'on ne possède aucun moyen de mesurer exactement la production annuelle d'un peuplement.

Il en résulte que la recherche de l'âge qui assure la plus grande production ligneuse ne peut se faire que par l'observation directe des volumes divisés par leur âge, comparée à la production observée sur les huit ou dix dernières années, celle-ci étant prise comme équivalente à la production annuelle. On n'a pas encore trouvé une règle de calcul fondée sur des mesures fournies par le peuplement.

La solution du problème est encore plus indéterminée quand l'exploitation est composée, c'est-à-dire, quand le peuplement fournit à la fois des éclaircies et une coupe principale. Il faut naturellement faire entrer le produit de ces éclaircies dans le calcul de la production moyenne: or, on ne connait pas la loi de formation du volume fourni par les éclaircies; il peut arriver qu'une éclaircie trop 
forte avance considérablement l'époque du maximum dans la production moyenne etque celui-ci soit ainsi dû au fait de l'homme plutôt qu'à celui de la nature. Il peut arriver, aussi, que les éclaircies étant bien opérées, leur volume complète celui de la production principale de telle sorte que la production moyenne reste constante jusqu'à l'âge de l'éclaircissement naturel dû à une vieillesse avancée, et qu'il n'y ait ainsi aucun intérêt à rechercher s'il se rencontre un maximum de rendement qui n'existerait pas, avant la vieillesse extrême. Les observations faisant défaut il est impossible de se prononcer autrement sur ce problème. Son utilité est, du reste, peu considérable puisque le propriétaire d'une exploitation à bois doit chercher beaucoup plus le maximum duproduit en argent que celui du rendement en matière: c'est moins la quantité que la qualité de la matière ligneuse qui lui importe.

No 105. - 20 Arbres. - Quand on recherche à quel âge il faut régler une exploitation d'arbres pour obtenir la production moyenne maxima, il faut faire attention qu'il s'agit de la production de l'arbre et non de celle du terrain; il faut, en d'autres termes, considérer que la production n'est pas rapportée à l'unité cle surface et que le terrain est, dans la forêt, librement accordé à l'arbre pour se développer.

Dans ces conditions, les quotients de la division du volume par l'âge fourniront des productions moyennes de plus en plus grandes et il n'y aura pas de maximum lorsque l'arbre vit d'une manière régulière. Le chêne donné en exemple au $n^{\circ} 100$ en fournit la démonstration facile à comprendre, puisque le terrain occupé grandit toujours. Il faut donc conclure que dans les exploitations d'arbres isolés, il n'y a pas de maximum de rendement autre que celui de l’âge le plus avancé. En d'autres termes, on obtiendra la plus grande quantité de matière, en coupant les arbres à leur maturité, cette époque de maturité étant considérée 
par tous les forestiers, comme l'âge le plus avancé possible où le bois reste sain. C'est à cette époque de maturité que. la production maxima est assurée pratiquement quand les arbres ont le terrain libre pour se développer.

Le calcul conduit, du reste, à la même conclusion : Le volume d'un arbre est $\mathrm{V}=f \mathrm{HD}^{2}$, formule dans laquelle $f$ est un facteur de forme, $H$ la lianteur totale et $\mathrm{D}$ le diamètre à $1^{\mathrm{m}}, 30 \mathrm{du}$ sol. Si on fait $\mathrm{D}=\alpha, n, \alpha$ étant l'épaisseur moyenne de la couche annuelle mesurée sur le diamètre, et $n$ l'âge de l'arbre, le volume peut se mettre sous la forme $f \mathrm{H} x^{2} n^{2}$, ou sous la forme $\mathrm{K} n^{2}$, en faisant $f \mathrm{H} \alpha^{2}=\mathrm{K}$. La production annuelle est, suivant la règle donnée au $\mathrm{n}^{0} \underset{\mathrm{K} n^{2}}{98} \mathrm{~K}^{2} \times \frac{1}{{ }^{1 / 2} n}=2 \mathrm{~K} n$, et la production moyenue à $n$ ans est $\frac{\mathrm{K}^{-} n^{2}}{n}=\mathrm{K} n$; nous avons vu, $\mathrm{n}^{\circ} 104$, que cette production moyenne est maxima quand elle est égale à la production anuuelle; or, cela ne peut pas arriver, puisque $\mathrm{K} n$ ne peut pas ètre égal à $2 \mathrm{~K} n$, les variations de $f$, de $\mathrm{H}$ et de $\varkappa^{2}$ étant impuissantes à produire ce résultat quand la marche de la végétation n'a pas d'accidents.

Cependant il existe des cas où un maximum de production ligneuse pourrait se présenter sans qu’il y ait dépérissement et sans que les arbres soient arrivés à leur âge habituel de maturité. Des arbres sont implantés sur un sol fertile et d'épaisseur régulière au delà de laquelle se présente une couche plus compacte que les racines ont peine à pénétrer. Ces arbres, sans présenter de signes extérieurs de dépérissement, fabriquent moins de bois, ce qui apparaît à l'épaisseur des couches annuelles derenues très minces. Cette circonstance se rencontre fréquemment dans les sapinières des montagnes granitiques des Vosgges, où les souches présentent l'aspect indiqué à la figure 24. Il est naturel de se demander, alors, s'il n'y a pas un moment où il y a intérét à abaltre les arbres pour réaliser la production ligneuse la plus grande possible et quel moyen on possède pour déterminer ce moment.

Il est certain que dans une situation pareille, plus fré- 
quente qu'on ne le pense généralement, les quotients successifs de la division du volume par l'âge indiqueront le moment du fléchissement daus la production.

Mais ce moyen est fort délicat à employer, parce qu'il est difficile d'avoir des arbres de divers âges, vivant dans des conditions identiques et parce qu'il faut sacrifier un assez grand nombre de tiges. Il nous paraît préférable de se décider d'après les indications des souches d'arbres abattus.

La production moyenne maxima est obtenue quand la conche annuelle (mesurée sur le diamètre ou sur le rayon) aura une épaisseur moindre que la moitié de l'épaisseur moyenne des couches antérieures.

Jusqu'à 120 ans, par exemple, l'épaisseur moyenne des couches annuelles, en diamètre, est de 5 millimètres; à partir de cet âge, on constate que la couche annuelle n'a plus que 2 millimètres (1 millimètre sur le rayon), c'est à 120 ans qu'il faut abattre les arbres pour obtenir le maximum de rendement en matière.

Désignons par $\mathrm{D}$ le diamètre à $n$ ans et par $(\mathrm{D}+\delta)$ le diamètre à $n+1$ ans : il faut calculer la couche $\delta$ de telle sorte qu'elle apporte à l'arbre un volume égal ou inférieur à la production moyenne $\frac{\mathrm{V}}{n}$, car nous avons vu que cette production moyenne est maxima quand le volume dû à $\delta$ (production annuelle) lui est égal ou, à plus forte raison, inférieur (fig. 24).

Le volume de l'arbre à $n$ ans étant $f \mathrm{H} \mathrm{D}^{2}$; on peut admettre que le facteur de forme $f$ et la hauteur $\mathrm{H}$ ne changeront pas de $n$ à $n+1$ ans. Le volume à $n+1$ ans sera $f \mathrm{H}(\mathrm{D}+\delta)^{2}$. La production de l'année $n+1$ sera

$$
f \mathrm{H}(\mathrm{D}+\delta)^{2}-f \mathrm{H} \mathrm{D}^{2}=2 f \mathrm{HD} \delta+f \mathrm{H}^{2}
$$

Il faut que cette feuille soit égale ou plus petite que la production moyenne

$$
2 f \mathrm{HD} \delta+f \mathrm{H}^{2}=\frac{f \mathrm{HD}^{2}}{n}
$$

Divisant les deux termes par $f \mathrm{H} \mathrm{D}^{2}$, il faut que

$$
\frac{20}{\mathrm{D}}+\frac{\delta^{2}}{\mathrm{D}^{2}}=\frac{1}{n}
$$


négligeant la quantité très petite $\frac{\delta^{2}}{\bar{D}^{2}}$, il faut que

$$
\frac{2 \delta}{\mathrm{D}}=\frac{1}{n} \text { ou que } \delta=1 / 2 \frac{\mathrm{D}}{n}
$$

Ainsi, lorsqu'une couche sera égale à la moitié de la couche moyenne antérieure, le maximum de production moyenne sera atteint et, à plus forte raison, quand la couche sera plus faible que la moitié de l'épaisseur moyenne des couches antérieures.

La quantité négligée n'influe en rien sur le résultat, quand l'âge $n$ est assez grand, car le calcul exact aurait donné

$$
\grave{o}=\frac{\mathrm{D}}{n}(\sqrt{n(n+1)}-n)\left({ }^{1}\right)
$$

et on aurait trouvé pour :

$$
\begin{aligned}
& n=30 \quad \text { o }=\frac{\mathrm{D}}{n} \times 0,496 \\
& n=60 \quad \text { o }=\frac{\mathrm{D}}{n} \times 0,498 \\
& n=90 \quad \text { o }=\frac{\mathrm{D}}{n} \times 0,499 \\
& n=120 \text { ○ }=\frac{\mathrm{D}}{n} \times 0,500
\end{aligned}
$$

Cette règle doit être appliquée avec un certain discernement: il ne faudrait pas condamner des arbres à être coupés à 64 ans, par exemple, parce qu'on a remarqué sur les souches voisines que la soixante-cinquième couche concentrique a une épaisseur moindre que la moitié de l'épaisseur moyenne des 64 couches antérieures. Ce fait peut être accidentel et provenir d'une golée, d'une sécheresse exceptionnelle ou d'une invasion d'insectes. Il faut examiner si cette diminution dans l'épaisseur des couches se maintient d'une manière soutenue et sans se relever plus tard; c'est dire qu'il faut opérer le calcul sur la couche moyenne d'un certain nombre d'années. Il peut même arriver que la croissance d'un arbre ayant été

(1) Calcul de N. Niegrer, inspecteur des forêts à Nancy. 
ralentie à différentes époques, il y aurait plusieurs maxima de production dans son rendement total. On ne saurait régler l'âge d'une exploitation sur des accidents de cette nature. C'est au sylviculteur à s'appliquer à les éviter autant que possible.

$3^{\circ}$ Obsemation. - Il ne faudrait pas appliquer aux peuplements formés de tiges d'un seul âge, ce que nous venons de dire pour l'arbre isolé. Celui-ci s'accroît constamment, et quand il vit d'une manière régulière, la quantité moyenne de matière fabriquée s'accroît toujours sans présenter de maximum. Il en est de même des arbres qui constituent le peuplement, car leur fabrication, quoique ralentie par le voisinage des tiges voisines, suit la même loi générale, mais le nombre de ces tiges diminue graduellement avec l'âge. Cette diminution du nombre compense-t-elle l'augmentation de fabrication individuelle, et la production collective en devient-elle constante? Les tables allemandes affirment le contraire et la physiologie végétale, qui fonde la fabrication du bois sur le développement foliacé, conclut, comme elles, à l'existence d'un maximum de production arrivant plus tard et au moment de I'éclaircissement du feuillage, causé, à un âge avancé, par la diminution du nombre d'arbres.

Toutefois, lorsque un peuplement présente des tiges dont les souches offrent, dans leur ensemble, les caractères indiqués à la figure 24, c'est-à-dire lorsque l'épaisseur des couches devient à un certain âge plus faible que la demi-épaisseur moyenne des couches antérieures, on peut être certain que le peuplement est arrivé à l'âg'e du maximum de sa production ligneuse, car la diminution naturelle du nombre des tiges ne peut qu'accentuer davantage la décroissance de la fabrication collective. Les ouvriers de la fabrication ayant atteint leur maximum, leur réunion l'atteint aussi puisqu'elle n’augmente plus en nombre. 
Voilà donc plusieurs circonstances qui peuvent produire dans un peuplement d'un seul âge, une et méme plusieurs époques de maximum dans la production : éclaircissement causé par l'âge avancé dans la puissance du feuillage ; diminution notable d'épaisseur de la couche concentrique des tiges; - affaiblissement de l'accroissement en hauteur; - le tout sans compter l'effet physiologique dî aux éclaircies qui peut retarder, avancer ou compenser la production générale. La science forestière présente encore de nombreuses questions inconnues et les stations d'expériences auront fort à travailler pour les résoudre. L'économiste peut heureusement l'en affranchir : car si l'exploitation forestière avait pour but unique de fixer du carbone et de fabriquer de la matière ligneuse, abstraction faite de ses qualités, l'âge du maximum de production serait la plus importante des questions à résoudre. Mais c'est bien plus la qualité que le poids ou le volume qu'on cherche à produire, en d'autres termes, le volume produit doit étre formé par du bois de dimensions telles qu'on puisse en retirer des marchandises utiles. Si le maximum en quantité arrive, par exemple, à l'âgre où le bois ne peut être utilisé qu'en chauffage, l'exploitant aura toujours profit à lui préférer l'âge qui lui procure du bois de service se vendant deux ou trois fois plus cher, quoique se produisant en quantité un peu moindre. Il en résulte que le problème du maximum de production, considéré jadis comme très important, n’a, en réalité, qu'une utilité très secondaire.

Il en est de même, mais pour un autre motif, dans les forêts jardinées : celles-ci présentent un état naturellement aménagé; la production annuelle y est constante quand on exploite toujours au même âge; mais si on modifie le terme de l'exploitation, la production peut très bien changer : un jardinage réglé à 50 ans, par exemple, pourra offrir une production annuelle toute différente que 
celui qui aurait été réglé à 100 ans en mêmes essences et en même sol. Faut-il rechercher, par des expériences délicates et nombreuses, l'âge où le massif jardiné présente le maximum de production? Non, car l'exploitation ne porte pas sur le massif; elle n'a pour but que des arbres isolés par la sélection naturelle qui s'opérera dans le massif de remplissage. Or, nous avons vu que la production moyenne d'une exploitation en arbres isolés auxquels le terrain ne manque pas, va toujours en augmentant. Il en résulte que dans les forêts jardinées, l'exploitant recueillera le maximum de production en réglant le terme de l'exploitation à l'âg'e le plus avancé où le bois reste sain, c'est-à-dire à l'âge de la maturité des arbres. Les forêts jardinées échappent ainsi, par leur nature, aux difficultés théoriques du maximum de production dans les forêts de peuplements d'un seul âge.

\section{§3. - Possibilité.}

Nos 106. - Définition : trois formes de possibilité.

107. - Exemples de possibilités pour les divers modes d'exploitation.

108. - Conditions auxquelles la possibilité doit satisfaire.

109. - Origine et différents aspects de la possibilité.

110. - Mesure de la possibilité.

111. - Production possible : production de l'aménagement et produit réalisé.

112. - La production résultant de la possibilité fournit-elle toujours la production réelle de la forêt?

113. - Néthodes inventées pour fournir la production réelle dans le jardinage.

$\mathrm{N}^{\circ}$ 106. - Les explications précédentes ont montré que le revenu d'une forêt est exactement formé par la production annuelle des tiges et des peuplements qui la constituent. Or, l'ignorance où l'on est des lois de la végétation ne permet pas de mesurer sûrement cette production annuelle; d'ailleurs, quand bien même on y arriverait, il 
ne serait pas facile de réaliser cette production sur tous les points de la forêt d'une manière complète et efficace. Enfin, il ne suffit pas de recueillir exactement la production, il faut, en outre, la percevoir en bois ayant les dimensions correspondant à l'âge et au but de l'exploitation. On a donc imaginé des procédés pratiques pour percevoir le revenu de l'exploitation. La possibitité est l'expression pratique du revenu, c'est la forme suivant laquelle on le percoit.

Il y a trois modes de perception du revenu, c'est-à-dire trois natures de possibilité.

Par contenance;

Par pieds d'arbres;

Par volume.

Les deux premières sont les plus anciennes et romontent aux origines de l'art forestier; elles correspondent aux deux éléments constitutifs des exploitations forestières: le peuplement et l'arbre ( $\left.{ }^{0} 56\right)$. La troisième est d'origine plus récente et nous est arrivée au début du siècle par importation allemande.

La possibilité par contenance s'applique aux exploitations de peuplements ; c'est ainsi que dans un taillis réglé à 30 ans, la possibilité est le 1/30 de la contenance; c'est ainsi, encore, que les éclaircies faites dans les peuplements d'un seul âge ont une possibilité par contenance.

La possibilité par pierls d'arbres s'applique aux forêts jardinées et aux futaies sur taillis.

La possibilité par volume est pratiquée surtout pour exprimer le revenu des futaies pleines exploitées à long terme; après avoir groupé les bois les plus âgés dans un quartier spécial (affectation) destiné à fournir le revenu pendant un certain nombre d'années, on exprime ce revenu par le quotient de la division du volume total par ce nombre d'années.

On combine souvent entre elles ces trois formes de pos- 
sibilité pour préciser davantage l'expression du revenu. C'est ainsi que la possibilité du furetage est une contenance sur laquelle on coupe toutes les tiges ayant la dimension fixée. Le revenu des forêts jardinées qui s'exprime par pieds l'arbres peut être limité par une contenance ou par un volume. - Les taillis sous futaie ont une possibilité par contenance pour le taillis et par pieds d'arbres pour la futaie. - Enfin, dans les forêts jardinées, on exprime parfois la possibilité par les trois formes: on dit, par exemple, qu'elle sera de tant de pieds d'arbres pris sur le $1 / 10$ de la contenance et sans dépasser tant de mètres cubes.

La possibilité par volume est le mode le moins parfait pour exprimer le revenu; il ne suffit pas, en effet, de déterminer quel volume on prendra chaque année; il faut, en outre, indiquer en quelle nature de bois le volume sera perçu, car le revenu en argent peut varier considérablement suivant la dimension des bois qui fournissent le volume déterminé. Aussi ce mode de possibilité n'est pratiqué qu'avec certaines précautions et de manière à s'affranchir autant que possible de ces inconvénients:

Une forêt de futaie pleine de 120 hectares a été divisée, par exemple, en quatre affectations de 30 hectares :

La $1^{\text {ro }}$ pour les bois de 90 à 120 ans.... 30 hectares.

\begin{tabular}{|c|c|c|c|c|c|}
\hline $\mathrm{La} 2^{\circ}$ & - & 60 à & 90 ans. . & 30 & - \\
\hline $\mathrm{La} 3^{\mathrm{e}}$ & - & 30 à & 60 ans..... & 30 & - \\
\hline $\mathrm{La} 4^{\circ}$ & - & 0 à & 30 ans..... & 30 & - \\
\hline
\end{tabular}

120 hectares.

On compte et on cube les bois de la $1^{\text {ro }}$ affectation; on trouve, par exemple, 14,400 mètres cubes; on y ajoute l'accroissement pendant 15 ans, soit 600 mètres cubes, ce qui fournit un total de 15,000 mètres cubes à réaliser en 30 ans. La possibilité est de :

$$
\frac{15000}{30}=500 \text { mètres cubes. }
$$

On voit que pour obtenir cette expression du revenu, on 
a dû se livrer à une appréciation de l'accroissement. Le volume concentré dans le quartier affecté aux bois les plus âgés fournira bien des marchandises correspondant à l'âge adopté ; mais ce volume, basé sur une appréciation de l'accroissement et sur une contenance qu'on peut composer arbitrairement avec les peuplements de la forêt, ne formera qu'une expression incertaine du revenu et il sera nécessaire de reviser cette possibilité à certains intervalles, par exemple, tous les 10 ans. Les possibilités par contenance et par pieds d'arbres échappent à cette incertitude et n'ont jamais besoin de revision.

$\mathrm{N}^{\circ}$ 107. - Il n'est pas sans intérêt de passer en revue les différents modes d'exploitation pour indiquer des exemples de possibilité et montrer la manière dont on exprime pratiquement le revenu dans les circonstances les plus habituelles.

A) Forêt de peuplements d'un seul âge :

$1^{\circ} 120$ hectares de taitlis ordinaire exploités à 30 ans. - Possibilité : 4 hectares de coupe à blanc estoc;

$2^{\circ} 480$ hectares de futaie pleine exploités à 160 ans et divisés en quatre affectations de 120 hectares. - Possibilité principale: 1/40 du volume actuel et de son accroissement pendant 20 ans des bois de la première affectation. - Possibitité intermédiaire : éclaircie de 3 hectares par an sur la deuxième affectation; éclaircie de 6 hectares par an sur la troisième affectation pour la parcourir deux fois dans la période de 40 ans; nettoiement de 6 hectares par an sur la première affectation avec la même condition.

$B)$ Forêts d'arbores isolés :

$1^{\circ} 90$ hectares de forêt furetée à rotation de 10 ans. Possibilité: 10 hectares de coupe annuelle en coupant les tiges de $0^{\mathrm{m}}, 30$ de tour à $1,30 \mathrm{du}$ sol et en laissant toutes celles de dimensions inférieures;

$2^{\circ} 10$ hectares de forêt traitée en jardinage simple à 
coupe annuelle réglée sur l'arbre de 0,60 de diamètre correspondant à 150 ans. - Possibilité : 12 arbres à couper cà et là et près de voisins plus jeunes pour les remplacer ;

$3^{\circ} 120$ hectares de forêt soumise au jardinage simple exploitée sur la même base et avec rotation de 10 ans. Possibilité: 12 hectares par an avec coupe de 144 arbres en jardinant.

La possibilité des forèts jardinées se détermine d'après la production moyenne à 150 ans. On calcule, par exemple, que un hectare peuplé de tiges de 150 ans offre un volume de 684 mètres cubes, soit par année moyenne

$$
\frac{684}{150}=4^{\mathrm{mc}}, 560
$$

L'arbre de 150 ans ayant $0^{\mathrm{m}}, 60$ de diamètre dans ce mode de traitement et cubant $3^{\mathrm{mc}}, 800$, on pourra couper par hectare et par an

$$
\frac{4,560}{3,800}=1,20
$$

soit pour une forêt de 120 hectares 144 arbres sur le coupon annuel.

Cette possibilité se calcule également par une manière diffèrente d'exprimer la même idée. Considérez en effet une suite aménagée de 150 hectares et contenant des peuplements âgés de 1 à 150 ans, à raison d'un hectare pour chaque âge. Calculez le nombre de tiges que peut présenter un hectare plein d'arbres de 150 ans ; en faisant ce calcul par' des places d'essai ou par le couvert directement mesuré sur un certain nombre d'arbres, vous obtiendrez 180 arbres par exemple. Supposez que tous les éléments de la forêt soient confusément mélangés comme ils le sont dans le jardinage. Cet état de mélange n'empèchera pas de recueillir chaque année dans une forêt de 150 hectares 180 arbres de 150 ans et dans une forèt de 120 hectares

$$
\begin{gathered}
150: 180:: 120: x \\
x=144 \text { arbres. }
\end{gathered}
$$

On voit que la détermination du nombre de tiges sur l'hectare de 150 ans équivaut à l'évaluation de la production, car

$$
180 \times 3,800=684 \text { mètres cubes. }
$$


$4^{\circ} 120$ hectares de forêt traitée en jardinage composé avec rotation de 10 ans. - Possibilité : 12 hectares de coupe annuelle avec 396 tiges à couper çà et là et en jardinant, savoir : 144 arbres de sciage, 48 doubles pannes, 84 pannes simples, 120 chevrons.

Une possibilité ainsi composée ne peut s'établir que d'une manière approximative et par analogie avec les produits qu'on pourrait retirer d'une exploitation en futaie pleine régulièrement disposée. Supposons, pour donner idée du mode de calcul, que les sapins de sciage aient 150 ans, les doubles pannes 120 ans, les pannes 90 ans et les clevrons 60 ans. Si on considère une forêt de 150 hectares en peuplements pleins régulièrement aménagés, on pourra déterminer avec assez d'exactitude le nombre de tiges sur l'hectare exploitable, ainsi que le nombre des tiges à recueillir par suite du déchet naturel à 120 , à 90 et à 60 ans. On a calculé, par exemple, que dans cette forèt de 150 hectares, on peut réaliser chaque année 810 tiges, savoir :

A 150 ans 180 tiges, soit 1,2 par hectare.
A $120-120-0,8-$
A $90-210-1,4-$
A $60-\frac{300}{810}-\frac{2,}{5,2}$

Pour une forêt de 120 hectares, la réalisation proportionnelle fournirait les résultats suivants :

120 hectares $\times 1,2=144$ arbres.
$120-\times 0,8=96$ doubles pannes.
$120-\times 1,4=168$ pannes simples.
$120-\times 2=\frac{240}{648}$ chevrons.

Les 120 hectares jardinés sont évidemment capables de contenir le même nombre de tiges que la futaie pleine formée de peuplements d'un seul âge méthodiquement disposés sur des contenances égales. Mais rien n'indique qu'ils les contiennent à l'état disponible, car elles sont confusément mélangées, de telle sorte que chaque hectare peut présenter les âges les plus divers. Cet état spécial des forèts jardinées impose une grande morération dans la jouissance, parce que :

$1^{\circ} \mathrm{Il} \mathrm{y}$ a des tiges tour à tour dominées et dominantes, et avec des recouvrements de l'une par l'autre, dans une proportion qu'il est impossible de déterminer $(f i g .6)$; 
$2^{\circ}$ Il est certain que toutes les tiges de la forèt de peuplement peuvent tenir dans la même contenance de forêt jardinée, mais nul ne sait si la nature produit dans celle-ci les tiges de tous âges dans la même proportion que celle résultant de la conception théorique d'un hectare affecté ì chaque âge caractéristique de la futaie pleine.

Il faut donc tenir compte de cette double inconnue en réduisant les résultats du calcul précédent dans une proportion que nul ne connait encore et que nous avons évaluée à moitié dans l'exemple précédent.

144 arbres de sciage.

48 doubles pannes.

84 pannes simples.

120 chevrons.

396

Cette infériorité du jardinage est plus apparente que réelle: le propriétaire peut toujours réaliser les tiges intermédiaires dont la surabondance viendrait à se manifester, c'est une appréciation culturale qui se rencontre également dans les éclaircies des futaies pleines. L'infériorité ne porte ainsi que sur les produits intermédiaires, et le jardinage présente assez d'avantages par la facilité de sa l'égénération et par sa résistance aux intempéries des régions montagneuses pour qu'on lui passe ce léger défaut. Celui-ci ne consiste d'ailleurs qu'à observer' le sage principe : la modération est la règle du jardinage.

$5^{\circ}$ Une forêt de 120 hectares est traitée en fitaie étagée à la rotation de 30 ans. - Possibilité: 4 hectares à couper à tire-aire en laissant 390 arbres par hectare (3 gros, 69 moyens, 198 petits et 120 brins).

Cette possibilité qui est réglée par un calcul de différence, indiqué $n^{\circ} 7 \%$, ne peut guère s'exprimer que par les catégories habituelles de grosseur.

C'est au propriétaire à observer les règles culturales qui ont pour but de graduer les âges en laissant à la coupe la faculté de se régénérer par semence et au massif étagé celle de se reformer en état convenable en laissant tout ce qui est en bon état de végétation et en ne coupant que les tiges incapables de prospérer jusqu'à la coupe suivante.

C) Forêts mixtes d'arbres et de peuplements :

$1^{\circ} 120$ hectares sont traités en taillis sous futaie 
simple à l'âge de 30 ans. - Possibitité: 4 hectares de coupe à tire-aire avec réserve de 150 baliveaux par hectare, en brins de semence ou, à défaut, en bons sujets de taillis et choisis principalement en chênes.

$2^{\circ}$ Une forêt de 120 hectares est traitée en taillis sous futcie composée à l'âge de 30 ans. - Possibitité: 4 hectares de coupe à tire-aire avec réserve de 110 arbres par hectare (60 baliveaux, 40 modernes et 10 anciens) choisis principalement en chênes et en hêtres.

$3^{\circ}$ Une pineraie de 120 hectares est exploitée à 50 ans avec arbres de réserve destinés à être coupés à l'âge de 100 ans. - Possibilité : coupe de 2 hectares 40 ares à tire-aire avec réserve de 40 pins par hectare choisis parmi les arbres les mieux venants.

$\mathrm{N}^{\circ}$ 108. - In voit que l'expression pratique du revenu ne peut s'opérer que par un petit nombre de types, de sorte que les modes de possibilité sont, ainsi, assez limités.

Une bonne possibilité doit, en effet, satisfaire à plusieurs conditions :

$1^{\circ}$ Elle doit être simpie, facile à appliquer et se prêter ainsi à une administration commode du domaine ;

$2^{\circ}$ Elle doit réaliser exactement le revenu et fournir la production annuelle de toute la forêt avec l'approximation que comporte la nature des choses;

$3^{\circ}$ Elle doit, par l'effet de son seul mécanisme, constituer l'exploitation aménagée sans que le propriétaire ait pour ainsi dire à s'en inquiéter; pour produire ce résultat, la possibilité doit être naturellement en harmonie avec les éléments constitutifs de l'exploitation.

Ces trois conditions essentielles sont entièrement réalisées par les deux plus anciens modes de possibilité : la contenance et le nombre de pieds d'arbres.

1. Examinez une exploitation de taillis ordinaire qu'on veut 
régler à 30 ans. La possibilité est le $1 / 30$ de la contenance. Par le seul fait de la coupe, ayant chaque année le $1 / 30$ de la contenance, l'exploitation se constituera par 30 éléments âgés de 1 à 30 ans et le revenu sera exactement formé de toute la production aunuelle comme le montre la figure 25 : la possibilité forme et constitue l'aménagement par son seul mécanisme ;

$2^{\circ}$ Dans une futaie sur taillis dont le plan de balivage est de 60 baliveaux, 40 modernes et 10 anciens, la possibilité est, comme nous l'avons vu $\mathrm{n}^{0} \mathbf{7 7}$, de 20 modernes, 30 anciens et 10 bis-anciens par hectare de coupe. Si on pratique régulièrement cette possibilité et si on laisse, par conséquent, sur chaque hectare de coupe le plan de balivage adopté, on recueillera exactement toute la production annuelle des arbres de la forêt et de plus celle-ci se constituera en exploitation aménagée, c'est-à-dire capable de fournir régulièrement dans l'avenir le même revenu annuel;

$3^{\circ}$ Il en est de même dans une forêt jardinée: supposons qu'on ait évalué la production moyenne par hectare à $4^{\mathrm{mc}}, 1 / 2$ par an et qu'on désire régler l'exploitation en arbres de $0^{\text {m }}, 60$ de diamètre, fournissant $3^{\mathrm{mc}}, 800$, le revenu par hectare étant égal à la production moyenne $\left(\mathrm{n}^{\circ} 102\right)$, la possibilité sera :

$$
\frac{4^{\mathrm{mc}}, 500}{3,800}=1,20
$$

La possibilité d'une forêt de 100 hectares serait de 120 arbres à prendre, par exemple, sur la coupe de 10 hectares de cette forêt, divisée en 10 coupons de 10 hectares. Par le seul fait de la coupe de ces 120 arbres chaque année, il arrivera ceci : si le coupon en tour de coupe est pauvre en bois de tous âges, c'est-à-dire, si le matériel aménagé présente des insuffisances dans sa constitution, les 120 arbres étant de petite dimension n'enlèveront pas les $450^{\mathrm{mc}}$ qui forment la production, le coupon s'enrichira de l'économie faite. $\mathrm{Si}$, au contraire, le coupon est riche en matériel, les 120 arbres étant de dimensions plus fortes enlèveront plus que la production et le matériel se réduira en conséquence. Il en résulte que la forêt tendra à se modeler sur le matériel qui correspond à un état régulièrement jardiné et constitué en vue de la dimension adoptée.

La possibilité constituera l'exploitation par son seul mécanisme et la production annuelle sera perçue dans la limite compatible avec la nature de l'exploitation. 
Ces trois conditions fondamentales de toute possibilité sont bien moins assurées dans l'expression du revenu par le volume.

Cette possibilité, introduite dans la gestion des forêts par le désir légitime d'obtenir une plus grande exactitude, exige une administration intelligente et soigneuse, des revisions périodiques, des cubages exactement faits et une appréciation du volume futur que notre ignorance des lois de la régétation rend incertaine. Si les affectations sont exactement régénérées dans l'ordre établi, la possibilité constituera l'aménagement, mais ce résultat sera dû bien plutôt à la marche suivie dans l'ordre des affectations qu'au jeu de la possibilité par volume. Ces affectations elles-mêmes formées par une appréciation plus ou moins arbitraire peuvent être composées de telle sorte qu'on ne sera jamais certain si le volume de la coupe représente exactement la production annuelle. Nous reconnaissons que dans les grandes forêts exploitées en futaies pleines la méthode de possibilité par volume est encore la plus simplifiée, et qu'il n'était pas possible de faire mieux à raison des exigences culturales de la régénération : ce n'est pas une raison pour dissimuler les imperfections de cette possibilité,car pour certaines forêts, elle ne paraît pas avoir assuré la constance et l'égalité du revenu plus que tout autre mode.

$\mathrm{N}^{\circ}$ 109. - Le mot possibilité est très ancien; on le trouve dans l'ordonnance de 1669 et même dans des actes antérieurs. Les articles 65 et 119 du Code forestier en ont fait un terme légal et la règle de jouissance des usagers dans les forêts: la délivrance qui leur est due peut toujours être ramenée à la possibilité de la forêt et ne peut la dépasser, car il lui est interdit de la dégrader : le maximum de son droit est ainsi le revenu ou son expression pratique, la possibilité. 
Il est digne de remarque que dans tous les actes réglementaires anciens, que le Code forestier n'a fait que reproduire, la possibilité se trouve toujour's précisée par l'état de la forêt: "L'exercice des droits d'usage pourra " toujours être réduit suivant l'état et la possibilité des "forêts" (C. f. 65).

Ces deux mots signifient que selon la nature de l'exploitation, l'expression pratique du revenu sera conforme à son traitement et que la possibilité sera par contenance, par exemple, dans les taillis ordinaires et par pieds d'arbres dans les futaies sur taillis.

L'étymologie du mot possibilité est également digne de remarque : elle indique la capacité de la forêt, le "revenu dont celle-ci est susceptible sans être ni dégradée, ni améliorée, de telle façon qu'elle reste dans le même état et que la végétation répare ce que la jouissance lui enlève. ")

Dominés par cette origine, certains auteurs ont défini la possibilité : la quantité de matière qu'on peut retiver" annuellement d'une forêt sous la condition de la maintenir dans le même état. Cette définition, qui a le tort de prendre l'effet pour la cause, n'est exacte que dans le seul cas où l'expression du revenu a pour but de maintenir la forêt dans le même état et d'en recueillir l'usufruit économique.

L'économie forestière envisage les choses plus largement et considère la possibilité comme la simple expression pratique du revenu, étant entendu que le revenu demandé à une forêt peut avoir pour but soit de la maintenir dans le même état, soit de l'améliorer, soit enfin, d'en diminuer la valeur.

Lorsqu'on veut rendre une exploitation plus intensive, et que, par exemple, on reut passer d'une exploitation aménagée à 30 ans à une autre réglée à 90 ans, sur le même terrain, il faudra bien faire des économies sur la 
production moyenne pour constituer le matériel de 1 à 90 qui est la base de l'exploitation. Dans cet exemple, analysé $n^{\circ} 95$, la possibilité de trois hectares se réduit à un hectare et la production fixée à 150 mètres cubes au début s'améliore progressivement jusqu'à atteindre 450 mètres cubes à 90 ans, moment à partir duquel elle se maintiendra constante et fixée à 450 mètres cubes.

Inversement, si on veut adopter un âge plus faible, par exemple passer d'un taillis aménagé à 30 ans à une exploitation aménagée à 25 ans, le revenu sera plus considérable que la production et la possibilité sera temporairement accrue, c'est-à-dire que pendant 25 ans, la forêt coupée par $25^{\circ}$ de la contenance, fournira un revenu plus élevé que dans l'avenir.

Cet état de variation en plus ou en moins dans le revenu de la forêt, selon qu'on voudra rendre l'exploitation plus extensive ou plus intensive, ne sera toutefois que temporaire et subsistera pendant la durée de l'âge adopté pour l'exploitation. Après cette durée, la possibilité reprendra le caractère indiqué par l'étymologie et deviendra l'expression du revenu constant dont la forêt est capable.

On a cru devoir différencier, par les mots, ces diverses phases de l'expression pratique du revenu: A la possibitité temporaire recueillie pendant la durée de la constitution de l'aménagement, on a opposé la possibilité définitive qui sera recueillie après cette constitution. D'autres ont proposé les mots taxe ou taxation de l'aménagement pour exprimer cet état temporaire et ont réservé le terme de possibilité pour le cas oì il faut déterminer le revenu sans changer la valeur de la forêt.

Nous pensons qu'on peut se dispenser de ce mot nouveau, puisqu'il s'agit d'un simple but assigné au revenu et non d'une modification dans l'idée même. La possibilité n'est que l'expression pratique du revenu et il suffit de la 
caractériser en opposant la possibitité de l'aménagement à la possibitité ordinaire qui a pour but de laisser la forêt dans le même état.

$\mathrm{N}^{\circ}$ 110. - Des possibilités par contenance, par pieds d'arbres et par volume ne fournissent pas des résultats comparables. Or, quand on veut comparer les exploitations entre elles, ou établir la comptabilité d'une forêt pour en raisonner en connaissance de cause, on est obligé d'appliquer à la possibilité une commune mesure qui est le volume en mètres cubes pleins. On préfère naturellement cette évaluation du volume au stère qui exprime une marchandise et au mètre cube au quart ou au cinquième qui sont des manières conventionnelles et commerciales de mesurer, en certaines localités, les bois de service.

Exemples : $1^{\circ} 30$ hectares de taillis ordinaire, aménagés à 30 ans, ont pour possibilité un hectare de 30 ans. Cette coupe produisant 300 stères de rondin et de charbonnette à 2 stères pour 1 mètre cube, la mesure de la possibilité est 150 mètres cubes.

$2^{\circ} 30$ hectares de taillis sous futaie ont une possibilité de 1 hectare de 30 ans avec réserve de 60 baliveaux, 40 modernes et 10 anciens. La mesure de la possibilité sera 169 mètres cubes, ainsi détaillés :

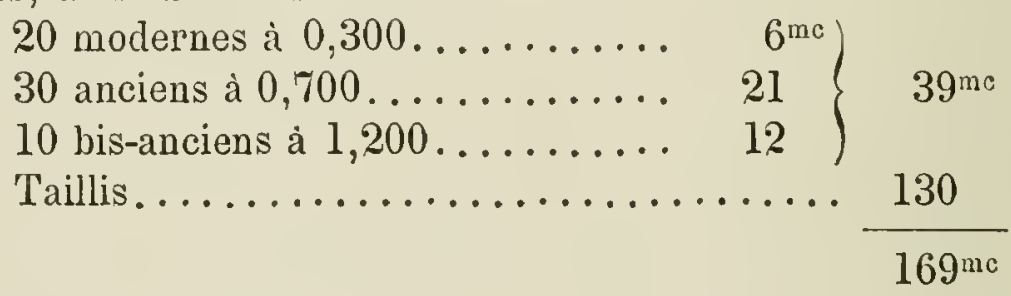

Il est souvent utile de rapporter à l'hectare la mesure de la possibilité, on obtient ainsi la production réelle dans les limites où la possibilité peut l'exprimer.

Dans les exemples ci-dessus la production de l'exploitation en taillis est, par hectare :

$$
\frac{150}{30^{\mathrm{h}}}=5^{\mathrm{mc}}
$$


Celle du taillis sous futaie

$$
\frac{169}{30^{\mathrm{h}}}=5^{\mathrm{mc}}, 633
$$

On peut distinguer la production due à la possibilité principale et celle provenant des produits intermédiaires dans les forèts qui comportent des éclaircies.

$\mathrm{N}^{\circ}$ 111. - Le chiffre de la production par hectare est la note caractéristique de l'exploitation que le propriétaire doit avoir toujours sous les yeux pour se rendre compte de sa gestion et pour se décider en connaissance de cause aux améliorations qu'il y a lieu d'introduire, aux épargnes qu'on veut entreprendle et aux anticipations qu'on peut se permettre sans trop compromettre l'avenir.

Mais il importe de se rendre un compte exact de la signification de ce produit par hectare.

Nous avons vu qu'il y a lieu de distinguer la possibitité ordinaire, laissant l'immeuble dans le même état, de la possibilité de l'aménagement, qui a pour but de modifier l'intensité de l'exploitation. La même distinction doit être faite dans la production à l'hectare.

On veut, par exemple, rendre plus intensive l'exploitation du taillis sous futaie précédent; on ne coupe que 5 anciens au lieu de 10 pour avoir dans l'avenir 5̆ antiques à réaliser. La possibilité temporaire de cet aménągement sera un hectare de taillis avec réserve de 115 arbres ( 60 baliveaux, 40 modernes, 10 anciens et 5 bis-anciens) et abaudon de 55 arbres ( 20 modernes, 30 anciens, 5 bis-anciens). La mesure de cette possibilité sera 163 mètres cubes.

20 modernes à $0,300 \ldots \ldots \ldots \ldots$

30 anciens à $0,700 \ldots \ldots \ldots \ldots$.

5 bis-anciens à $1,200 \ldots \ldots \ldots \ldots$.

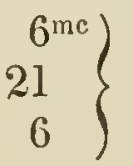

Taillis..................... 130

$163^{\mathrm{mc}}$

et par hectare

$$
\frac{163}{30}=5^{\mathrm{mc}}, 433
$$

Après 30 ans, l'exploitation, rendue plus intensive, aura une 
possibilité de 1 hectare de taillis avec réserve de 115 arbres ( 60 baliveaux, 40 modernes, 10 anciens, 5 bis-anciens) et abandon de 60 arbres (20 modernes, 30 anciens, 5 bis-anciens, 5 antiques).

La mesure de la possibilité sera 170 mètres cubes.

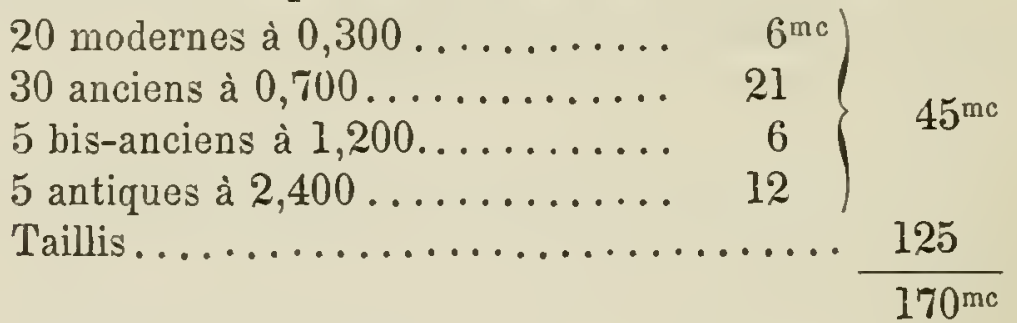

La production par hectare :

$$
\frac{170}{30}=5^{\mathrm{mc}}, 666
$$

Cet exemple appelle une double observation :

La première est que l'économie à faire pour passer à une exploitation plus intensive est de

$$
5^{\mathrm{mc}}, 633-5^{\mathrm{mc}}, 433=0^{\mathrm{mc}}, 200 .
$$

Il faut épargner par hectare et chaque année un cinquième de mètre cube pendant 30 ans pour constituer la nouvelle exploitation.

La seconde est que l'exploitation étant montée et établie à un revenu de 5 classes d'âge, la production par hectare ne sera pas sensiblement différente de celle qui était fournie par l'ancienne exploitation (nº 110) à 4 classes d'âge $(5,666$ et 5,633$)$. C'est par la qualité des produits et par le revenu en argent que se manifestera l'amélioration de l'exploitation.

La raison en est fort simple : le bois est le produit des agents naturels; ceux-ci n'ayant pas changé et le développement foliacé étant à peu près le même sur un terrain complètement garni de tiges, il est fort probable que la production ne varie pas sensiblement avec l'âge et la disposition des peuplements tant que la vie végétale se maintient dans ses conditions habituelles.

Cette double observation faite, on concoit combien il 
est utile que le propriétaire ait constamment sous les yeux pour se guider dans sa gestion les 3 chiffres suivants ramenés à l'hectare :

La production possible;

La production de l'aménagement;

Le produit réalisé.

La production possible est la mesure ramenée à l'hectare de la possibilité que comporte le mode de traitement de l'exploitation forestière sur le terrain et avec les essences de la forêt. On la calculera en appliquant la conception théorique de l'exploitation aux données locales et en s'éclairant de l'expérience des forêts voisines. Malheureusement, le peu de renseignements que nous possédons sur la marche de la végétation donnera toujours à cette détermination un caractère plus expérimental que scientifique. Peu importe, au surplus, puisqu'il ne s'agit que d'un renseignement destiné à éclairer la gestion. Il est des cas où le chiffre de la production possible devient un renseignement indispensable pour la bonne conduite d'une exploitation de forêt: dans les grandes futaies pleines où l'affectation des bois mûrs peut être formée par des parties plus ou moins riches en matériel, il est nécessaire de mettre la production possible en regard de la production de l'aménagement pour qu'on puisse savoir si la possibilité, telle qu'elle a été réglée pour celui-ci, demande trop ou trop peu à la forêt.

La production de l'aménagement est celle qui r'ésulte de la mesure de la possibilité calculée en vue d'un but à atteindre, c'est-à-dire d'un aménagement projeté. Son caractère temporaire durera jusqu'à ce que le but poursuivi soit atteint et que l'exploitation soit constituée pour l'âge choisi. Cet état dure fort longtemps et la production de l'aménagement ne se confond avec la production possible que quand : $1^{\circ}$ le but est atteint; $2^{\circ}$ on veut maintenir la forêt dans le même état. 
Le produit réalisé est le résultat rapporté à l'hectare de toutes les coupes faites pendant l'année, coupes qui peuvent varier avec les besoins du propriétaire, les accidents et les circonstances imprévues.

Dans l'exemple précédent, la production possible peut être fixée au chiffre de................... $5^{\text {mc }}, 633$

La production de l'aménagement est de....... $5^{\mathrm{mc}}, 433$

La production réalisée varie chaque année..... Mémoire.

Les deux premiers chiffres résultent d'évaluations et de calculs; ils figurent en tête des papiers de la gestion et de la comptabilité que doit tenir tout propriétaire soucieux de sa fortune, et désireux de s'intéresser à sa forêt. L'étude de cette comptabilité doit être un chapitre important de l'application de la théorie à la forêt réelle.

$\mathrm{N}^{\circ}$ 112. - La production possible est-elle la même que la production réelle du sol? En d'autres termes, la production résultant de la possibilité spéciale à un mode d'exploitation atteint-elle un chiffre égal à la quantité de matière ligneuse réellement produite par tous les éléments de la forêt ? Cette question est très digne d'intérêt, elle a occupé et occupera, longtemps encore, l'attention des forestiers désireux de savoir si les procédés actuels de possibilité sont de nature à réaliser entièrement la production du sol quand l'exploitation est régulièrement constituée.

Il est certain que plusieurs modes de possibilité sont de nature à fournir au propriétaire toute la production du sol et que la production possible est ainsi égale à celle de la forêt.

Quand on règle un taillis ordinaire à 30 ans, il est manifeste que la coupe du trentième de la surface assure chaque année au propriétaire tout l'accroissement annuel du matériel sans en rien excepter et qu'ainsi cette coupe réalise toute la production réelle. La figure $\mathbf{2 5}$ démontre cet effet mieux que tout raisonnement. 
Il en est de même quand on constitue une exploitation aménagee d'arbres sur taillis. La disposition 'de la figure 25 s'applique aussi bien à l'arbre qu'à l'hectare de coupe, car le sol occupé par les arbres est une surface constante prise sur la partie affectée à la coupe du taillis et dont il est facile d'évaluer la mesure.

Dans les exploitations de taillis ordinaire, de taillis sous futaie, de futaies étagées, et en général dans toutes celles où les arbres sont isolés par la main de l'homme, la production possible est bien égale à la production réelle; mais il n'en est pas de même dans les exploitations d'arbres isolés par la sélection naturelle, telle que le jardinage et le furetage; il y a un remplissage nécessaire qui grandit et s'accroît chaque année et dont les produits doivent être réalisés d'une manière plus ou moins approximative. La possibilité par pieds d'arbres, spéciale à ces forêts, ne permet que d'approcher plus ou moins de la production réelle, même quand l'exploitation est composée, attendu qu'on est loin de connaître la marche de la végétation et notamment la loj du nombre des tiges naturellement produites.

Il en est de même des forêts de futaie pleine: la possibilité principale réalise assez exactement la production réelle des produits principaux, si le quartier qui leur est affecté est convenablement peuplé et déterminé; mais la possibilité des éclaircies n'étant réglée que par contenance, il en résulte que les produits intermédiaires ne sont recueillis qu'au point de vue cultural et suivant des règles de pure appréciation.

La possibilité de ces coupes ne fournit donc la production r'éelle que d'une manière approchée et arbitraire; on n'est jamais certain de recueillir le déchet normal causé par l'âge dans le nombre des tiges. La production due à la possibilité ne représente la production réelle que suivant des inspirations culturales toujours incertaines. 
No 113. - On s'est ingénié à remédier à l'imperfection des méthodes pour les forêts jardinées et même pour les forêts de peuplements plus ou moins irréguliers. De nombreux auteurs mus par un sentiment très louable ont cherché des procédés pour déterminer la possibilité dans le but de faire réaliser au propriétaire toute la production réelle de la forêt.

Nous ne pouvons entrer dans l'examen détaillé des différentes méthodes qui ont été proposées; il n'est pas inutile cependant d'en apprécier le système.

Naturellement c'est par une possibilité exprimée en volume que ces auteurs ont cherché la solution du problème. Ils se sont appliqués à déterminer exactement la quantité de mètres cubes qu'on doit exploiter pour réaliser complètement la production réelle.

Les différents systèmes qui ont été présentés avec plus ou moins d'art se résument dans une prescription dont voici le caractère général :

Comptez et cubez toutes les tiges de la forêt; évaluez la quantité dont elle s'accroìt chaque année, par exemple 1,000 mètres cubes (cette évaluation se fait, à titre provisoire, suivant les uns d'après le volume du matériel nécessaire à l'exploitation, suivant les autres d'après l'accroissement probable des arbres). Ce chiffre de 1,000 mètres cubes obtenu ainsi par une simple appréciation sera exploité chaque année suivant les indications culturales, mais au bout de 10 ans, par exemple, on compte et on cube de nouveau tous les arbres de la forêt; en réunissant le volume du début au volume exploité, et en lui comparant le volume de ce deuxième cubage, on déduit le taux de l'accroissement, on constate si la forêt est restée dans le mème état, si elle s'est améliorée ou amoindrie.

On règle alors, mais cette fois en connaissance de cause, le volume de la coupe annuelle. On le réduit (soit 900 mètres cubes) ou on l'augmente (1,200 mètres cubes) selon qu'on veut améliorer l'exploitation ou la maintenir dans le même état. Chaque dix ans on recommence le même cubage de toute la forêt et on est ainsi en mesure d'apprécier exactement le but poursuivi, à la condition toutefois de tenir une exacte comptabilité de tout ce que l'on coupe entre chaque inventaire. 
Que des procédés de cette nature soient exacts et rationnels, nous ne saurions le méconnaître, mais il est permis d'en relever les imperfections économiques:

$1^{\circ}$ Cette exactitude, cette sûreté de direction vers le but assigné à une exploitation ne sont acquises qu'au prix de coûteux inventaires, difficiles à pratiquer dans une grande gestion de forêt et à la condition d'une comptabilité difficile à tenir avec l'esprit de suite nécessaire. La simplicité désirable dans toute méthode de gestion n'est certainement pas réalisée;

$2^{\circ}$ Cette possibilité par volume ne satisfait pas à la condition essentielle et nécessaire d'une bonne méthode; celle-ci doit constituer l'aménagement par son seul mécanisme; elle doit, en d'autres termes, disposer les peuplements de telle sorte que, par le seul fait de la coupe régulièrement pratiquée, l'exploitation aménagée se trouve constituée;

Qu'un propriétaire faisant ses coupes d'après ses seules inspirations soit désireux de se renseigner au bout d'un certain temps, sur l'effet de ces coupes, au moyen du cubage général de sa forêt, qu'il puise dans ce cubage des renseignements sur l'état de sa fortune et des appréciations pour l'avenir, rien de mieux ni de plus naturel! Mais on conviendra qu'il n'y a dans cette manière d'opérer qu'un procédé et non une méthode d'aménagement!

$3^{\circ}$ Il ne suffit pas de déterminer le volume dont la forêt s'est exactement accrue, il faut encore indiquer de quelle manière on le prendra, c'est-à-rlire en quelles dimensions d'arbres le volume sera fourni et en quelle proportion il sera composé en bois des divers âges qui peuplentla forêt. Théoriquement, si tous les âges existent convenablement échelonnés suivant leur loi naturelle, il faudrait prendre la possibilité en bois de chaque âge et pour une fraction correspondante au nombre de tiges de chaque catégorie. Comme il est impossible d'arriver à cette exactitude tléo.. 
rique, on est obligé de faire une hypothèse sur la proportion qui existe entre le volume des petits arbres, des moyens et des gros, par exemple :

50 pour $0 / 0$ de gros bois,

30 - de bois moyens,

30 - de petits bois,

et de prescrire qu'on prendra chaque année la possibilité fixée (900 mètres cubes) autani que possible suivant cette proportion, sauf à la rectifier d'après les indications fournies par les inventaires ultérieurs.

Ainsi, voilà le résultat de tant de peines : on a fixé exactement l'accroissement, c'est-à-dire la production réelle, mais pour appliquer la possibilité qui en résulte, il faut substituer aux appréciations culturales des anciennes méthodes, une autre appréciation de la manière dont les arbres sont associés dans la nature. C'est déplacer la difficulté et non la résoudre. A chaque inventaire, en effet, l'accroissement pourra être modifié ; on aura trop ou pas assez perçu et, en fin de compte, on n'aura pas réalisé davantage la production réelle, ce qui était cependant le but du procédé.

On s'épuisera en efforts pour chercher la perfection sans jamais l'atteindre. Dans toute exploitation de forêts, il y a, en effet, la considération culturale qui ne peut s'évaluer mathématiquement, car elle résulte seulement de l'observation des faits. Il faut laisser à la sylviculture la place quilui convient, et limiter les écarts de sa pratique; on peut modifier l'aspect sous lequel on envisage les exigences culturales, on ne saurait jamais s'en affranchir et on ne s'écarte jamais sans péril des règles tracées par la longue expérience du passé. Il est donc bien probable que de longtemps encore les vieilles méthodes dictées par cette expérience resteront la règle des exploitations de forêts. Nous ne saurions toutefois blâmer des recherches conseillées par une légitime aspiration vers le mieux; 
elles seront toujours un intéressant sujet d'études pour les propriétaires ayant l'amour de la forêt.

\section{Article 2.}

\section{Produit en argent.}

Nos 114. - Rente de la forèt, définition.

115. - La rente augmente arec l'âge d'exploitation.

116. - Conditions nécessaires pour cette augmentation de la rente.

117. - Combien rapportent les forèts dans telle localité.

118. - Fausse délinition de la rente.

119. - A quel âge faut-il régler l'exploitation pour obtenir la plus grande rente?

120. - Terme d'exploitation convenant à l'État.

121. - Utilité de la rente pour la gestion d'un domaine forestier.

No 114. - Dans une exploitation forestière, le revenu en matière (production annuelle) s'exprime par la possibilité ; celle-ci se mesure par le volume.

Rapportée à l'unité de contenance, la mesure de la possibilité fournit la production possible par hectare et par an.

Le revenu en argent prend un nom particulier qui est la rente de la forêt ou de l'exploitation. Celle-ci, rapportée à l'unité de surface, forme la rente moyenne par hectare et, par an.

La rente est ainsi le revenu en argent de l'exploitation, déduction faite des dépenses de création, de fabrication et de récolte. Cette définition est en harmonie avec celle qui a été introduite par Ricardo dans l'économie politique générale. Comme la fabrication est toujours gratuite dans les forêts et comme la création est presque toujours naturelle, la rente de la forêt se réduit, en réalité, au revenu en argent. 
Cependant, on distingue la rente brute et la rente nette.

Elle est brute quand du produit en argent on défalque uniquement les frais de la récolte, c'est-à-dire, quand on constate ce qui revient au propriétaire exploitant, s'il vendait ses coupes sur pied.

Elle est nette quand on défalque, en outre, les frais de garde, d'administration et d'impôt.

50 hectares de pineraie aménagée à 50 ans, fournissent chaque année:

Une coupe principale à 50 ans valant... 2600

Une éclaircie à 40 ans........... 400 》

Une éclaircie à 30 ans............ 300 »

Une éclaircie à 20 ans............. 60 》

Chasse et menus produits.......... 100 p

La rente brute de l'exploitation est de. 3460 ”

$\mathrm{Si}$ on dépense chaque année 150 francs en frais de garde, d'administration et d'impôt, la rente nette est

La rente brute par hectare est

$$
3460-150=3310
$$

$$
\frac{3460}{50^{\text {hect }}}=69^{\mathrm{r}} 20
$$

La rente nette par hectare est

$$
\frac{3310}{50^{\mathrm{hect}}}=66^{\mathrm{r}} 20
$$

Lorsque l'exploitation est périodique, on a pour but de rechercher non plus la rente annuelle, mais la rente par année moyenne. Le calcul s'effectue de la manière suivante pour tenir compte des produits naturellement annuels et des charges.

50 hectares de pineraie exploités périodiquement à 50 ans fournissent en 50 ans :

50 hectares de coupe principale...... 130000

50 - de $3^{\circ}$ éclaircie........ 20000 "

50 - de $2^{\circ}$ éclaircie......... 15000 »

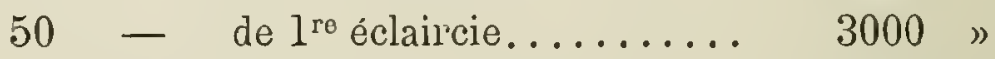

50 produits annuels de 100 francs..... $\frac{5000 \text { " }}{1730000}$ 
La rente brute est par année moyenne

et par hectare : $69^{\mathrm{r}} 20$

$$
\frac{173000}{50^{\text {ans }}}=3460^{\text {f }}
$$

La rente nette est obtenue en défalquant 50 années de charges à 150 francs, soit 7,500 francs.

$$
173000-7500=165500
$$

Elle est, ainsi, de :

et par hectare : $66^{\mathrm{f}} 20$

$$
\frac{165500}{50^{\text {ans }}}=3310^{\mathrm{f}}
$$

Il est évident que si la rente de l'exploitation aménagée exprime bien le véritable revenu annuel, celle de l'exploitation périodique ne correspond pas aux profits réels que l'exploitant en retire; il faudrait, en effet, ajouter aux produits annuels (chasses et menus produits) les intérêts capitalisés et agir de même, mais en sens inverse, pour les charges. Ce sont des annuités qui doivent entrer par le calcul de leur montant dans le produit perçu. Les produits d'éclaircies devraient également être grossis de leurs intérêts si on voulait calculer les sommes réellement produites au propriétaire par l'exploitation périodique; mais il ne s'agit pas d'un pareil calcul, on cherche uniquement la production en argent fournie par année moyenıe en produits forestiers et non en résultats de placements. La rente par année moyenne n'a pas d'autre signification, et à cet égard, elle doit être égale à la rente annuelle de l'exploitation aménagée.

Le calcul de la rente donne lieu à la même observation que celle faite au $\mathrm{n}^{\circ} 99$, pour la production en matière; il doit s'effectuer sur la durée de l'exploitation et non sur le temps pendant lequel les produits ont été acquis.

$\mathrm{N}^{\circ}$ 115. - Nous avons vu que la production en matière est à peu près constante dans les forêts, quel que soit l'âge 
de l'exploitation. La rente par hectare augmente au contraire toujours avec l'âge, en d'autres termes, la production moyenne en argent va constamment en augmentant à mesure qu'on avance l'âge d'exploitation.

Voici des produits principaux relevés dans des pineraies du centre de la France avec les rentes correspondantes:

\begin{tabular}{|c|c|c|c|c|c|}
\hline 20 ans & produ & $t 450^{\mathrm{f}}$ & ) & rente & $22^{\mathrm{f}} 50$ \\
\hline $30-$ & - & 900 & » & - & 30 \\
\hline $40-$ & - & 1500 & $»$ & - & 3750 \\
\hline $50-$ & - & 2600 & " & - & 52 \\
\hline $60-$ & - & 3600 & " & - & 60 \\
\hline $70-$ & - & 5000 & » & - & 7140 \\
\hline $80-$ & - & 6000 & D & - & 75 \\
\hline
\end{tabular}

Cette augmentation graduelle de la rente est la caractéristique des exploitations forestières : plus celles-ci sont intensives, plus elles fabriquent des marchandises meilleures et plus utiles. Cela tient à ce que le prix d'un mètre cube de gros bois fournit des produits plus variés et plus considérables que le même volume en petit bois. La forêt continue à fabriquer tous les ans le même volume de matière, mais ce volume se vend chaque année à un prix plus élevé.

La statistique forestière dressée en 1878 constate que la rente des forêts de l'État est en moyenne de $40 \mathrm{fr} .25$ par hectare. Ce chiffre n'est qu'une moyenne composée de toutes les forêts les plus diverses.

La rente dépasse notablement cette moyenne dans les conservations du Nord et de l'Est (Amiens, 89 fr. - Epinal, $71 \mathrm{fr}$ - Nancy, 58 fr.). Par contre, la rente s'abaisse au-dessous de la moyenne dans les forêts du Midi; elle tombe au-dessous de 5 fr. par hectare et par an dans 6 conservations formant 16 départements (TouTouse, Pau, Bordeaux, Ajaccio, Chambéry, Nice). Cet abaissement de la rente tient non seulement à ce que les exploitations sont réglées à des âges plus faibles que dans le Nord, mais en outre, à ce que les forêts livrées au 
pâturage sont en mauvais état ou situées en des régions mal outillées en moyens de vidange. On voit quel vaste champ d'améliorations s'offre de ce côté aux patriotiques efforts des forestiers.

$N^{\circ}$ 116. - L'augmentation de la rente avec l'âg'e n'est vraie, comme loi économique, que lorsque les moyens de vidange et l'outillage industriel de la localité sont capables d'assurer aux produits forestier's toute leur valeur d'utilisation.

En effet, lorsqu'on examine le prix auquel on vend 1 mètre cube de bois, on remarque bien vite que plusieurs éléments interviennent pour le fixer et qu'il y a des lois pour la valeur comme il en existe pour tous les faits économiques.

En principe, plus le bois dont le mètre cube est formé a de fortes dimensions, plus on en retire de marchandises précieuses (grosses poutres, larges planches, etc.) et plus on en obtient de marchandises de débit ordinaire (sabots, merrains, chevrons, etc.), et, enfin, plus les déchets de fabrication sont diminués : c'est la loi de l'utilisation.

Or, il s'en faut que celle-ci soit toujours la règle du prix.

Ici, les voies de vidange et les moyens de transport manquent; les voitures et les attelages du pays ne sont pas assez forts pour sortir les grosses pièces de la forêt. Le mètre cube en arbres de $0^{\mathrm{m}}, 70$ ne se paiera pas plus au propriétaire que le même rolume en arbres de $0^{\mathrm{m}}, 50$ de diamètre; il deviendra plus coûteux à l'acheteur et parfois même il sera dédaigné : c'est la loi du transport qui agit en sens inverse de la loi d'utilisation.

Ailleurs, les moyens de débiter le bois sont peu perfectionnés; les scieries ne sont pas disposées pour la fabrication des charpentes équarries à vives arêtes; tous les arbres sont débités en planches de longueur uniforme: 
c'est la loi des débouchés qui fait encore obstacle à celle de l'utilisation.

Là, enfin, le commerce des bois est mal établi ou exercé par quelques marchands qui s'entendent entre eux. Les forêts sont trop peu importantes dans la région pour donner naissance au grand commerce : c'est la loi de la concurrence qui produit encore le même effet, car il est digne de remarque que les bois ont le plus de valeur dans les pays les plus riches en forêts.

Sans doute, ces divers obstacles à la loi de l'utilisation économique sont temporaires; le progrès est en tout, mais le propriétaire doit compter avec tous les faits et régler son exploitation suivant ces données locales, sauf à tenir compte des circonstances qu'il est en sa puissance de modifier, telles que la viabilité et les moyens de transport.

$\mathrm{N}^{0}$ 117. - Combien rapportent les forêts dans telle localité?

Voilà une question qui est posée bien souvent et à laquelle les explications précédentes montrent qu'il est à peu près impossible de répondre si l'âge d'exploitation n'est pas indiqué.

Un forestièr exercé, connaissant le terrain et les essences qui peuplent les forêts, pourra toujours dire avec assez d'approximation que les foréts produisent, par exemple, 5 mètres cubes par hectare et par an. L'âge auquel on exploite les forêts influe peu sur la production en matière.

Mais les forêts qui sont capables de produire 5 mètres cubes par hectare peuvent rapporter 15, 20 ou 25 francs par hectare et par an si l'âge d'exploitation ne permet d'obtenir que de la charbonnette et du rondin. De pareilles différences dans la production en argent se manifestent dans les plus modestes taillis qu'un observateur su- 
perficiel pourrait consilérer comme équivalents; à plus forte raison, la rente varie-t-elle dans des proportions énormes lorsque l'âge d'exploitation devient considérable, le prix du mètre cube pouvant varier de 3 à $50 \mathrm{fr}$. pour les bois dechêne de chauffage ou de service. Il est donc indispensable, quand on veut répondre à la question qui nous occupe, de se rendre un compte exact de la nature des essences et surtout de l'âge auquel les forêts sont habituellement exploitées.

Cette observation montre également que lorsqu'on indique la production d'une forêt, il est inutile de préciser l'âge de l'exploitation, mais l'indication de cet âge est absolument nécessaire pour se rendre compte de la rente et pour comparer entre elles les exploitations dont on veut apprécier la valeur.

$\mathrm{N}^{\circ}$ 118. - Certains auteurs forestiers ont appelé rente la relation qui existe entre le produit et le capital ; ils ont confondu la rente (revenu en argent) avec le taux de ta rente (rapport du revenu au capital).

La valeur capitale des forèts de l'Etat ( 986,843 hectares) a étẻ estimée, en vertu de la loi de finances du 29. décembre 1873, à la somme approximative de $1,262,000,000$; il en résulte que la valeur moyenne de l'hectare est de 1,280 francs; si on compare à ce chiffre la rente brute, $40 \mathrm{fr}$. $25 \mathrm{c}$., constatee par la statistique de 1878 , on voit que le taux de la rente est de 3 fr. 15 c. pour 100 fr.

$$
\frac{40,25}{1280}=3^{\mathrm{r}} 15
$$

Le taux de la rente n'est pas autre chose que le taux de placement des fonds engagés darıs l'exploitation ( $\left.\mathrm{n}^{\circ} 122\right)$.

Cette confusion entre la rente et le taux du placement a amené les plus grandes erreurs, quand il s'est agi d'apprécier les écrits de ceux qui donnaient à la rente son sens économique. Ainsi, on a souvent mal compris, en France, le conseil donné par certains auteurs allemands d'exploi- 
ter les forêts de l'État à l'âge qui fournit la plus grande rente, c'est-à-dire le plus grand revenu annuel possible, ou le plus grand rendement en argent: jamais ces auteurs n'ont engagé à exploiter à l'âge qui assure le plus haut taux de la rente; jamais ils n'ont cherché que la rente absolue et non la relation du revenu au capital : engager les forestiers de l'État à faire produire aux forêts le revenu en matière qui est le plus avantageux par son prix et qui assure ainsi le maximum d'utilité est certainement le conseil économique que les forestiers français ont donné euxmêmes; on avait critiqué sans s'être entendu sur la définition de la rente.

$\mathrm{N}^{\circ}$ 119. - On demande à quel âge il faut exploiter une forêt pour obtenir le plus grand revenu en argent, c'est-àdire la plus grande rente?

Ce problème sur l'âge d'exploitation a la même solution, qu'il s'agisse d'une forêt aménagée ou d'une exploitation périodique; car le revenu en matière n'est autre que la production moyenne annuelle ou la production par année moyenne $\left(n^{\circ} 102\right)$. Il en est de même pour le revenu en argent, c'est-à-dire pour la rente forestière.

Dr, à mesure que l'arbre et le peuplement avancent en âge, les tiges acquièrent des dimensions plus fortes; le mètre cube, rendant plus de services, se paye plus cher; la rente forestière augmente continuellement avec l'âge tant que le bois reste sain.

Exemples: $1^{\circ}$ les chênes dont nous avons relevé les volumes ( $\left.\mathrm{n}^{\circ} 100\right)$, ont été estimés dans la même expertise aux prix ci-dessous.

En divisant le prix de l'arbre par son âge, on obtient la rente de l'arbre, abstraction faite du terrain occupé :

\begin{tabular}{|c|c|c|c|}
\hline 30 ans & $0^{f} 35$ & rente & \\
\hline $60-$ & 260 & - & 0 \\
\hline $90-$ & 13 & 一 & 0 \\
\hline $120-$ & $38 \quad$ & - & 0 \\
\hline
\end{tabular}




\begin{tabular}{|c|c|c|c|}
\hline 150 ans & $76^{\mathrm{f}}$ & $\eta$ & rente \\
\hline $180-$ & 132 & $»$ & - \\
\hline $210-$ & 204 & $"$ & - \\
\hline $240-$ & 298 & D & - \\
\hline
\end{tabular}

$2^{\circ}$ Les peuplements de sapin relevés par II. Lorey ont été estimés de la manière suivante, par hectare :

\begin{tabular}{|c|c|c|c|}
\hline 20 ans & $90^{\mathrm{f}}$ & ) & rente \\
\hline $30-$ & 270 & $\nu$ & - \\
\hline $40-$ & 630 & " & - \\
\hline $50-$ & 1200 & 》) & - \\
\hline $60-$ & 2000 & ) & - \\
\hline $70-$ & 3050 & D & - \\
\hline $80-$ & 4370 & ” & - \\
\hline $90-$ & 6060 & 》 & - \\
\hline $100-$ & 7930 & 》 & - \\
\hline $110-$ & 9900 & $D$ & - \\
\hline $120-$ & 11820 & 》 & - \\
\hline $130-$ & 13700 & 》 & - \\
\hline $40-$ & 15400 & $\eta$ & - \\
\hline
\end{tabular}

L'inspection de ces tableaux montre qu'il n'y a pas de maximum dans la rente et que le revenu le plus considérable en argent correspond à l'âge le plus avancé, tant que le bois reste propre à tous les usages.

Examinons d'abord les forêts de peuplements: il est certain que le maximum de la production en matière ne correspond en aucune facon au maximum du produit en argent. En d'autres termes, la diminution de production ligneuse qu'on a constatée dans les peuplements et qui, dans l'exemple ci-dessus, se manifeste à partir de 120 ans $\left(\mathrm{n}^{\circ} 100\right)$ n'est pas assez considérable pour influer sur l'augmentation de qualité et de cherté produite par un âge plus avancé. Mais cette augmentation graduelle de la rente n'existe qu'à une double condition: $1^{\circ}$ le prix du mètre cube doit augmenter graduellement et sans soubresauts; $2^{\circ}$ la forêt doit étre assez bien outillée en moyens de transport et de débit pour que les gros arbres recoivent toute leur valeur d'utilisation.

$1^{\circ}$ Dans les environs d'une mine, les bois de 35 ans se 
vendent 15 fr. le mètre cube en étais de mine; on constate que la dimension la plus favorable pour cette marchandise est obtenue vers 35 ans; au delà de cet âge, le bois devient trop gros et ne se vend plus que comme cliauffage, à $12 \mathrm{fr}$. le mètre cube.

Une forêt qui rapporte 4 mètres cubes par hectare et par

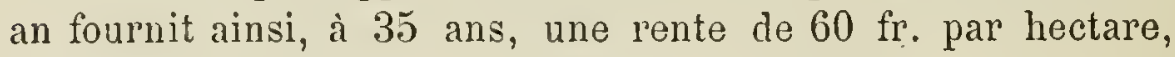
tandis qu'à 40 ans la rente s'abaisse à $48 \mathrm{fr}$.

$2^{\circ}$ Dans certaines localités, l'arbre perd de son prix quand sa dimension ne permet pas de le sortir de la forèt ou quand les modes de débit ne lui assurent pas dans le pays un emploi suffisant. En Algérie, les Arabes n'estiment que l'arbre qu'ils peuvent couper avec leur couteau; dans les Pyrénées, des hètres magnifiques ont été abandonnés sur le terrain faute de moyen de les utiliser en charbon, et, sans aller jusqu'à ces extrèmes, il n'y a pas bien longtemps que dans le Jura, un sapin de $0^{\mathrm{n}}, 70$ de diamètre se vendait moins cher que celui de $0^{\mathrm{m}}, 60$, à raison du mauvais état des scieries.

Il est certain que le maximum de la rente sera obtenu dans ces deux exemples à une époque bien antérieure à la maturité des peuplements; mais cet état ne sera que temporaire et cessera, soit par le seul effet du temps, soit par la création d'un bon outillage forestier. Qu'on laisse vieillir, en effet, la forêt qui produit un si bon rendement en étais de mine, le prix du mètre cube, après avoir fléchi un instant, ne tardera pas à augmenter quand les arbres seront capables de fournir d'autres marchandises recherchées (échalas, traverses, merrains, etc.) et le véritable maximum de la rente finira par se rencontrer à l'âge le plus avancé. Qu'on améliore les accès de la forêt de montagne pour permettre aux solides chariots d'aborder les coupes, qu'on installe dans les vallées des industries à débiter le bois et les gros arbres dédaignés à cause de leur poids prendront vite toute la valeur dont ils sont susceptibles; le maximum de la rente sera ainsi reculé à l’âgre de la maturité des arbres et la règle générale que nous aurons posée recevra sa confirmation.

En ce qui concerne les exploitations d'arbres isolés, le 
maximum de la rente n'apparaît aussi qu'à l'âge le plus avancé, quand les conditions de la végétation sont régulières et quand les accidents temporaires ne viennent pas y faire obstacle.

Il est évident qu'une exploitation d'arbres rẻglẻe à 240 ans, fournissant une production par année moyenne de $1 \mathrm{fr}$. 24, est plus avantageuse que celle qui permettrait de couper l'arbre de 60 ans 4 fois en 240 ans, car on n'aurait jamais qu'une production de 0,04 par année moyenne. En d'autres termes, celui qui laisse vivre un chène pendant 240 ans perçoit $298 \mathrm{fr}$. ; celui, au contraire qui abat l'arbre 4 fois en 240 ans ne recueille dans le même laps de temps que $2 \mathrm{fr}$. $60 \times 4=10^{\mathrm{f}} 40$.

Entre de pareils extrêmes, la différence de rente est trop considérable pour qu'il y ait le moindre doute à l'égard de l'âg'e qui assure le produit pécuniaire le plus considérable; mais si on considère des époques voisines dans la vie de l'arbre, par exemple 210 ans, où la rente est de $0 \mathrm{fr} .97$ et $240 \mathrm{ans}$, où elle est de $1 \mathrm{fr}$. 24, on peut se demander s'il n'arrive pas un moment où la couche concentrique annuellement fabriquée diminue assez d'épaisseur pour que l'augmentation de prix ne suffise pas à compenser l'abaissement de production ligneuse. Il y aurait alors un maximum de rente avant l'âge de la complète maturité de l'arbre. Nous pensons que ce cas est plus théorique que pratique, c'est-à-dire que la couche annuelle devrait être réduite à une épaisseur extrêmement faible etque le plus habituellement, pour ne pas dire toujours, la rente augmente constamment avec l'âge dans les exploitations d'arbres isolés chaque fois que la végétation suit sa marche naturelle.

Si on se reporte à l'exemple des chênes ci-dessus,il faut noter qu'on fait abstraction du sol occupé et que le terrain ne leur est jamais ménagé.En rapportant à l'hectare la production argent, on est certain que quatre suites aménagées de 1 à 60 ans occupent une surface bien moindre qu'une suite de 1 à 240 ans; mais si on connaissait ces surfaces, 
c'est-à-dire si on possédait des données expérimentales sur le couvert des arbres, il est très probable qu'on constaterait que les choses se passent comme pour les peuplements et que le maximum du produit-argent n'arrive qu'à la maturité, c'est-ì-dire à l'âge le plus avancé où le bois de l'arbre reste sain. Cette maturité est l'époque de la plus grande rente, sauf les cas exceptionnels où le maximum est atteint temporairement avant qu'elle soit arrivée.

$\mathrm{N}^{\circ}$ 120. - Il est très naturel qu'une commune ou un particulier règle son exploitation à l'âge qui lui assure en fait la rente la plus élevée, soit que ce maximum de la rente provienne de la plus-value notable et temporaire d'une marchandise spéciale, soit qu'il soit dû à un défaut de l'outillage de la localité, comme cela arrive dans les deux exemples du numéro précédent. Le propriétaire particulier aurait bien tort de reculer le terme de son exploitation et d'attendre de l'avenir des prix aussi avantageux que ceux qu’il trouve à un âge plus faible. Il n'est pas maître d'améliorer les conditions de viabilité du pays où sa forêt est située; il sera encore temps de modifier l'âge d'exploitation quand les conditions du marché ou de la viabilité auront changé.

L'État propriétaire de forêt aurait peut-être tort d'agir comme le particulier et d'arrêter son exploitation au terme d'un maximum accidentel de la rente. S'il possède, en effet, une forêt dans une région de mines où les bois d'étais se vendent plus cher que le bois de chauffage, les forêts de particuliers fourniront le plus souvent l'approvisionnement nécessaire à la mine. Les bois de plus fortes dimensions que l'État fabriquera, en reculant le terme de l'exploitation jusqu'à l'âg'e le plus avancé, sont susceptibles d'utilisations plus variées et plus nécessaires à l'ensemble de la population. Il n'est pas naturel que la forêt doma- 
niale, destinée par sa nature aux besoins généraux du travail national, soit affectée, en quelque sorte, à aider les besoins particulier's d'une industrie locale; alors surtout que la production de bois plus âgés fournit une rente plus élevée à la bourse commune. Des exemples pourraient être cités dans des départements où l'industrie du fer au bois donnait une forte valeur à la charbonnette. L'État a pu se laisser entraîner à favoriser ces forges et à profiter lui-même de cette plus-value en exploitant ses forêts en taillis à courts termes. Aujourd'hui que les forges au bois ont disparu, les produits de ces forêts ne se vendent plus et il faut les exploiter à un âge plus avancé. On aura ainsi perdu sur les taillis et il faudra se résigner à de longues épargnes pour avoir favorisé une industrie locale et avoir cherché un maximum accidentel de rente. Celui-ci n'a amené qu'un préjudice à la bourse commune et aux intérêts du travail général.

Quant aux forêts des régions inabordables où le manque de chemins empêche la valeur de se créer,l’État a les ressources nécessaires pour développer la viabilité; son devoir social l'y oblige en quelque sorte. Il aurait tort d'abaisser l'âg'e d'exploitation et de sacrifier des ressourses précieuses en gros bois par la raison qu'on ne peut en tirer pour le moment toute l'utilité possible. C'est à lui à créer la valeur là où elle n'existe pas encore et à régler ses exploitations de bois en vue de cet état futur, qui seul assurera au public l'utilité et le prix dont les produits forestiers sont susceptibles.

Concluons donc, qu'à moins de circonstances tout à fait exceptionnelles, le terme d'exploitation qui convient à l'État est celui de l'âge le plus avancé tant que le bois reste sain, parceque cet âge lui fournira le maximum de la rente, sinon immédiatement, au moins dans l'avenir. En principe, l’État doit exploiter ses forêts à l'âge qui fournit la jlus grande rente; ce maximum de la rente est 
en général atteint à la maturité des arbres, c'est-à-dire à l’âge le plus avancé où le bois reste sain. Quand cet âge ne fournit pas ce maximum de rente, il n'y a qu'à développer dans la localité les moyens d'assurer aux produits forestiers leur plus grande utilisation, pour que le prix qui en résulte assure à l'exploitation le revenu pécuniaire le plus élevé, et par suite la plus grande utilité au pays.

$\mathrm{N}^{\circ}$ 121. - Le chiffre de la rente produite chaque année parl'exploitation forme un renseignement très utile au propriétaire et indispensable à la gestion d'un ensemble de forêts.Il permet les comparaisons d'une année à l'autre, il éclaire sur les améliorations à introduire, indique leurs effets et montre les progrès de l'exploitation.

Ramenée à l'hectare, la rente devient le meilleur élément d'information pour le contrôle de la régie d'un vaste domaine forestier.

Que la comptabilité tenue par l'agent régisseur d'une forêt se termine chaque année par le produit par hectare en matière et en argent, par exemple:

$$
\begin{gathered}
\mathrm{P}=4^{\mathrm{mc}}, 650 \\
\mathrm{R}=38^{\mathrm{f}}, 50
\end{gathered}
$$

Que ces chiffres soient chaque année envoyés à la direction chargée de la gestion générale et, immédiatement, sans aucune hésitation, on se rendra compte des écarts dans le rendement et des points où il faut porter les dépenses: celles-ci doivent aller de préférence aux forêts les plus mal rentées et non à celles où la valeur est bien assise.

Le produit en matière indiquera si on ne demande pas trop à la forêt, si les prévisions d'un aménagement qu'on veut rendre plus intensif sont exactement suivies et dans quelles limites les écarts sont tolérables. Le produit en argent fournira des renseignements sur les prix 
obtenus par la gestion et sur les effets des améliorations faites dans l'outillage de la forêt. Dans les papiers de la gestion, c'est-à-dire dans les plans d'exploitation, on trouvera,pour éclairer le contrôle, le chiffre de la production réelle et celui de la production clue à la possibilité qui a été établie en vue d'un aménagement déterminé et ordonné. Les mêmes actes contiendront également le rendement pécuniaire espéré de l'aménagement. C'est à ces documents que seront comparés les produits réalisés en matière et en argent par les agents de la gestion. ("'est par ces chiffres que la direction du domaine aura le meilleur et le plus sûr élément de sa vérification. 


\section{TAUX DE L'EXPLOITATION}

Article 1er.

\section{Taux de placement.}

Nos 122. - Exploitations périodiques, - Taux de leur placement.

123. - Taux net du placement.

124. - Calcul du taux quand il y a des produits intermédiaires.

12כ. - Observations sur l'effet des éclaircies.

126. - Exploitations aménagées. - Formule du taux de placement.

127. - Estimations du rerenu et du matériel-bois.

128. - Évaluation du sol.

129. - Procédé erroné pour estimer les sols de forêts.

130. - Effet de l'augmentation de l'outillage sur le taux du placement.

131. - Comparaisons du taux de placement des exploitations périodiques el aménagées faites all même âge.

132. - Loi du taux des placements en forêts.

133. - Faiblesse du taux des exploitations à long terme.

134. - Age d'exploitation correspondant à un taux de placement déterminé.

13วั. - Age correspondant aı maximum du taux de placement.

136. - Conséquence économique de la diminution du taux de placement à mesure que l'âge d'exploitation augmente.

137. - Mesures légales d'encouragement aux futaies.

138. - Rôle des forêts de l'État : faire des arbres.

139. - Conséquences de l'aliénation des forêts de l'État.

$\mathrm{N}^{\circ}$ 122. - Exploitations périodiques. - Le plus grand souci et même le premier devoir de l'exploitant est de se rendre compte de la manière dont ses fonds sont placés dans l'exploitation. Le taux, qui est le loyer de l'unité de capital, intervient dans un certain nombre de questions d'économie forestière. La plus importante est 
celle qui consiste à chercher la mesure d'une exploitation en calculant le taux de placement des fonds qui y sont engagés.

Naturellement les fonds engagés sont : $1^{\circ}$ la valeur du sol ; $2^{\circ}$ celle du capital d'exploitation consistant dans l'outillage général et le matériel-bois. Il suffit de les mettre en présence du revenu par la relation mathématique des intérêts.

Nous calculerons d'abord le taux de placement d'une exploitation périodique.

La relation qui unit le fonds générateur $\mathrm{C}$ (sol garni de l'outillage et du capital-bois) au revenu périodique $R$ est :

$$
\mathrm{C}(1+x)^{n}=\mathrm{R}+\mathrm{C}
$$

Équation qui se résout par logarithmes :

$$
\log \cdot(1+x)=\frac{\log \cdot(\mathrm{R}+\mathrm{C})-\log \cdot \mathrm{C}}{n}
$$

Exemple: Un taillis ordinaire est exploité à 30 ans et produit à cet âge $980 \mathrm{fr}$. ; le sol vaut $420 \mathrm{fr}$. l'hectare et l'ensouchement $122 \mathrm{fr}$., de sorte que le capital engagé est 542 fr.

$$
\begin{aligned}
\log .(1+x) & =\frac{\log .1522-\log .542}{30} \\
x & =31 / 20 / 0
\end{aligned}
$$

La solution du problème serait également donnée d'une manière approximative en cherchant sur la ligne 30 du tarif I le facteur qui, multiplié par 542, fournirait le capital engagé augmenté de son revenu :

$$
542 \times 2,807=1521 \text { fr. } 39 .
$$

Le facteur 2,807 correspondant à $31 / 20 / 0$, on en déduit que la forêt est exploitée à ce taux. L'emploi de nos tarifs ne fournit naturellement qu'une approximation à $1 / 2 \quad 0 / 0$ près quand le résultat tombe entre 2 facteurs.

Il y a des exploitations périodiques d'arbres isolés dont le terrain n'est consacré à aucune autre culture (arbres des avenues et des haies de Normandie). Le calcul du taux de placement s'effectue par la même formule, mais il faut 
avoir soin d'y faire entrer, comme capital engagé, le terrain nécessaire à l'arbre exploitable, bien que le sol réellement occupé varie d'étendue à mesure que l'arbre augmente en âge. Ce terrain nécessaire au couvert final est, en effet, affecté à l'exploitation dès le début de celle-ci et forme ainsi le fonds générateur avec la valeur de la plantation.

Pour une exploitation à arbres de réserve, tels que les taillis sous futaie, il ne faudrait pas considérer, comme on l'a fait trop souvent, les modernes comme un groupe d'arbres se reproduisant tous les 60 ans, par exemple, les anciens tous les 90 ans et le taillis tous les 30 ans. L'exploitation ne s'opère pas de cette manière; il faut mettre le revenu réel résultant de l'abandon commandé par le plan de balivage en présence du capital engagé, qui est le sol garni du balivage initial, formant avec l'ensouchement le matériel-bois.

Exemple: Un taillis sous futaie exploité à 30 ans présente un balivage de :

60 baliveaux à $0^{\text {f }} 30 \ldots \ldots \ldots$ 18 $\left.18^{\text {\& }}\right)$

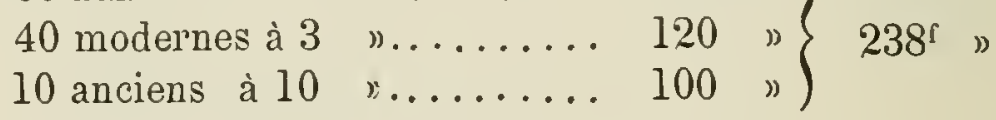

Le capital engagé est estimé $780 \mathrm{fr}$.

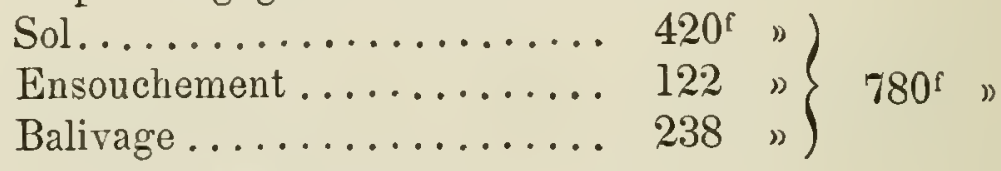

Le revenu est $1110 \mathrm{fr}$. :

20 modernes à $3^{\mathrm{f}}$ n............6.6.6. 60 »

30 anciens à 10 D........... 300 »

10 bis-anciens à $25 \quad n \ldots \ldots \ldots \ldots \ldots .250$ "

Taillis................... 500

Revenu.......... $\overline{1110,}$

Le taux de placement sera fourni par l'équation :

$$
\begin{gathered}
780(1+x)^{30}=1110+780 \\
x=30 / 0
\end{gathered}
$$

C'est de cette manière seule qu'on doit raisonner quand 
on se pose la question de savoir s'il y a avantage, au point de vue du placement, à conserver des arbres anciens ou bis-anciens sur les coupes d'un taillis sous futaie. On a trop souvent considéré ces arbres comme formant 'une exploitation individuelle, distincte de celle du taillis et à périodicité de 120 et 150 ans si le taillis s'exploite à 30 ans. Les arbres font corps avec l'exploitation en taillis et n'ont pas, alors, de taux de placement spécial. Les séparer de l'exploitation du taillis et les considérer comme une entreprise distincte, c'est s'exposer à un faux calcul et méconnaître les effets de l'augmentation du capital-bois, qui, dans les taillis sous futaie, a pour effel d'améliorer le rendement et de fournir un taux de placement convenable aux producteurs de gros arbres: une exploitation périodique à 150 ans en chênes isolés, sur un terrain dont on sacrifie la production agricole, ne fournit peut-être à son propriétaire qu'un placement à 1 ou à $1 / 20 / 0$. Les mêmes chênes dans un taillis sous futaie peuvent assurer, au contraire, un placement à $30 / 0$. La raison en est que dans le taillis sous futaie, ces chênes se perçoivent tous les 30 ans et non tous les 150 ans, grâce au matériel-bois dont le balivage a doté l'exploitation.

Aussi, il arrive que de nombreux particuliers propriétaires de taillis sous futaie peuvent être et sont, en effet, producteurs de gros bois sans que le taux de placement de leur fortune soit notablement abaissé, ce qui est un avantage à signaler en faveur des exploitations en taillis sous futaie composée.

$\mathrm{N}^{\circ}$ 123. - Le taux de placement ainsi calculé est brut.

Pour obtenir le taux net, il faut faire entrer dans le calcul les frais de garde, de gestion et d'impôts. A l'égard des impôts fonciers, il importe d'observer qu'ils sont bien plutôt une cliarge de l'individu à raison de sa fortune, qui 
pourrait être employée de toute autre manière, qu'une charge réelle due par l'immeuble.

Toulefois, si cette considération doit conduire à négliger l'impôt dans les estimations de forêts $\left({ }^{1}\right)$, elle ne saurait peser sur le calcul du taux net de placement; car l'exploitant doit tenir compte de tout ce qu'il paie pour sa propriété en frais de toute nature.

Ces charges (garde, gestion et impôts) sont annuelles, tandis que le produit de l'exploitation est périodique; or, il existe en même temps certains revenus annuels, tels que la chasse et les menus produits du sol qui entrent en déduction des frais. De sorte que, le revenu annuel est une quantité tantôt positive, tantôt négative, selon que les produits l'emportent sur les charges ou leur sont inférieurs. Le calcul du taux de placement résulte de l'équation suivante :

$$
\mathrm{C}=\frac{\mathrm{R}}{(1+x)^{n}-1} \pm \frac{a}{x}
$$

Le capital engagé $\mathrm{C}$ doit être considéré, en effet, comme formé par la réunion de 2 capitaux générateurs, l'un $c$ correspondant au produit périodique $\mathrm{R}$, l'autre $c^{\prime}$ correspondant au revenu annuel $a$. De la relation existant entre le capital $c$ et le revenu $\mathrm{R}$ acquis en $n$ ans :

on déduit

$$
c(1+x)^{n}=\mathrm{R}+c
$$

$$
c=\frac{\mathrm{R}}{(1+x)^{n}-1}
$$

De la relation entre le capital $c^{\prime}$ et le produit annuel $a$

$$
c^{\prime} x=a
$$

on déduit

$$
c^{\prime}=\frac{a}{x}
$$

De sorte que le capital engagé $\mathrm{C}$ est formé par la réunion des parties génératrices des l'evenus $\mathrm{R}$ et $a$, d'où résulte l'équation indiquée ci-dessus.

(1) Estimations concernant la prop:ieté forestière, p. 70. 
Il n'est pas possible d'exprimer $x$ par une formule en $\mathrm{C}, \mathrm{R}, a$ et $n$; mais le problème n'en est pas moins soluble par d'autres procédés.

Supposons que le taillis ordinaire du numéro précédent rapporte 980 avec un capital engagé de $542 \mathrm{fr}$.

Les produits de la chasse, de l'herbe et du bois mort sont évalués à $7 \mathrm{fr}$., et les charges de propriété à $4 \mathrm{fr}$. ; le produit annuel sera ainsi de $3 \mathrm{fr}$.

On essaie d'abord le calcul au taux de $31 / 2$ 0/0 à l'aide des tables:

$$
\begin{aligned}
980 \times 0,553 & \left.=542^{\mathrm{f}} \prime\right) \\
3 \times 28,5 \% & \left.=\frac{85}{627^{\mathrm{f}}}\right)
\end{aligned}
$$

Le capital ainsi trouvé étant supérjeur à $542 \mathrm{fr}$., le taux doit être plus avantageux que $31 / 20 / 0$. Si on fait l'essai avec $40 / 0$ et le tarif III, on obtient:

$$
\begin{gathered}
980 \times 0,445=436^{\mathrm{f}}{ }^{\prime \prime} \\
5 \times 25=\frac{75 \%}{\left.511^{\mathrm{f}}\right)}
\end{gathered}
$$

Le capital $511 \mathrm{fr}$. étant plus faible que $542 \mathrm{fr}$., on voit que lo taux de $40 / 0$ est trop fort et que le taux réel du placement est dans les environs de $3,800 / 0$.

On le calculerait exactement au moyen d'un essai intermédiaire opéré par logarithmes; mais cette approximation est bien suffisante dans la pratique.

Il y a des exploitations d'arbres implantés sur des terrains auxquels on demande une récolte agricole pendant le temps qui s'écoule entre la naissance de l'arbre et l'époque où on le coupe. (Arbres des pâturages, des prairies et des champs cultivés.) Le capital engagé est formé, comme nous l'avons vu $n^{\circ} 122$, par le terrain nécessaire à l'arbre exploitable; les récoltes agricoles sont annuelles mais naturellement décroissantes à mesure que l'arbre avance en âge; elles se calculent par une moyenne arbitrée entre la récolte au moment de la naissance (où elle est à son maximum) et celle du terme d'exploitation (où elle est mulle). Cette récolte moyenne annuelle entre 
dans la formule qui donne le taux du placement parmi les éléments de $a$ (produits et charges):

$$
\mathrm{C}=\frac{\mathrm{R}}{(1+x)^{n}-1} \pm \frac{a}{x}
$$

Dans cette formule, le second terme est positif quand l'exploitant forestier est en même temps propriétaire de la récolte; il est négatif quand la récolte appartient à un locataire que le propriétaire doit indemniser pour la diminution de revenu causée par le couvert de l'arbre. Il n'est pas possible de procéder autrement que par cette moyenne de la récolte agricole, car des annuités décroissantes n'ont pas de loi mathématique qui permette de les faire entrer dans le calcul. Le taillis qui croît sous les arbres d'une exploitation périodique en taillis sous futaie ne saurait être comparé à une récolte agricole, car celle-ci est annuelle, tandis que le taillis qui se développe sous le couvert d'un arbre quand il est jeune, s'étiole et ne sera jamais percu sur le terrain occupé par l'arbre de coupe qui, d'ailleurs, fait corps avec l'exploitation en taillis sous futaie.

$N^{\circ} 124$. - Lorsque l'exploitation périodique est composée, c'est-à-dire quand elle fournit, en outre du produit principal $\mathrm{R}$, des produits intermédiaires $r$ et $r^{\circ}$, dus aux éclaircies faites aux années $e$ et $e^{\prime}$, la formule qui sert à former le taux du placement est plus compliquée:

$$
\mathrm{C}=\frac{\mathrm{R}+r(1+x)^{n-e}+r^{\prime}(1+x)^{n-e^{\prime}}}{(1+x)^{n}-1} \pm \frac{a}{x}
$$

La démonstration se fait en considérant le capital engagé comme formé de plusieurs éléments :

$1^{\circ}$ Une partie $c$ correspondant au produit principal à $n$ ans ;

$$
c=\frac{\mathrm{R}}{(1+x)^{n}-1}
$$

$2^{\circ}$ Une partie $c^{\prime}$ correspondant au capital générateur de 
l'éclaircie $r$ faite à $e$ ans, dont la formule générale a été démontrée $\left(n^{0} 51\right)$ pour le cas où le bois a acquis $m$ ans d'âge. Ici, il est évident que le bois étant à l'âtge du début, $m=0$, et que cette partie de capital, génératrice de l'éclaircie, est:

$$
c^{\prime}=\frac{r^{2}(1+x)^{n-e}}{(1+x)^{n}-1}
$$

$3^{\circ}$ Une partie du capital $c^{\prime \prime}$ correspondant à l'éclaircie $r^{\prime}$ faite à $e^{\prime}$ ans dont l'expression est identique ;

$4^{\circ}$ La partie du capital $c^{\prime \prime \prime}$ génératrice du revenu annuel $a$ qui est:

$$
c^{\prime \prime \prime}= \pm \frac{a}{x}
$$

Le capital engagé total $C$ étant égal à la somme de ces quatre éléments

$$
\mathrm{C}=c+c^{\prime}+c^{\prime \prime} \pm c^{\prime \prime \prime}
$$

on obtient l'équation ci-dessus indiquée.

Cette formule, en apparence compliquée, n'est pas plus difficile à résoudre que la précédente : on s'aide des tarifs d'intérêts I et III, et on poursuit le calcul avec les tables de logarithmes quand on veut obtenir une exactitude absolue.

Exemple: Une futaie de hètre produit $4,000 \mathrm{fr}$. à $100 \mathrm{ans}$, avec une éclaircie de $400 \mathrm{fr}$. à 70 ans. Les produits annuels (chasse, herbe et bois mort) sont de $9 \mathrm{fr}$. Le terrain vaut $400 \mathrm{fr}$. l'hectare et le semis initial $100 \mathrm{fr}$., de sorte que le capital engagé est de $500 \mathrm{fr}$.

$\mathrm{Si}$ on essaie avec les tarifs le taux de $30 / 0$, on trouve que $\mathrm{C}=572 \mathrm{fr}$. ; ce chiffre étant plus grand que $500 \mathrm{fr}$., il en résulte que le taux de placement dépasse $30 / 0$.

L'essai fait avec $31 / 20 / 0$ donne $\mathrm{C}=427 \mathrm{fr}$., qui est plus faible que $500 \mathrm{fr}$.; le taux n'atteint donc pas $31 / 2$, il est intermédiaire entre 3 et $31 / 20 / 0$.

$\mathrm{Si}$ on veut obtenir une plus grande approximation, on fait le calcul par les logarithmes avec un taux intermédiaire, par exemple $3,200 / 0$. On trouve que la valeur de $\mathrm{C}=506 \mathrm{fr}$, qui est encore plus grande que $500 \mathrm{fr}$., mais qui s'en approche assez pour qu'on puisse dire que le taux de placement est de $3,200 / 0$, avec une approximation inférieure à $0 \mathrm{fr}$. 10 , car la valeur calculée avec 3,30 fournirait $477<500$ et ce taux serait trop fort. 
$\mathrm{N}^{\circ}$ 125. - L'exemple précédent donne lieu à certaines observations quand on effectue le calcul avec ou sans l'éclaircie et en comptant ou en négligeant les produits annuels.

Si on fait abstraction du revenu annuel, on voit que l'exploitation rapporte sans éclaircie ....... 2,20 $0 / 0$ avec l'éclaircie.................. 2,60 0/0

Si on tient compte du produit annuel, elle rapporte sans éclaircie.............. 3,10 0/0 avec l'éclaircie................ 3,20 0/0

$1^{\circ}$ Les produits annuels augmentent ainsi notablement le taux du placement des exploitations périodiques; cela explique la tendance des propriétaires à augmenter ces produits en louant les chasses, le bois mort et jusqu'au pâturage. Trop souvent, ils ne voient que le résultat immédiat sans assez se préoccuper du tort qu'ils causent à la végétation de la forêt et de la diminution de produits qui en résulte pour la coupe principale.

L’impôt et les frais de régie agissent de la même manière sur le taux, mais en sens inverse; leur exagération est une cause notable d'abaissement du taux de placement.

$2^{\circ}$ Les éclaircies n'augmentent pas beaucoup le taux du placement à moins qu'on ne les répète à des intervalles rapprochés, auquel cas on risque de diminuer le produit principal.

La raison de cette faible influence des éclaircies sur le taux général du placement est que les sommes venant de ces coupes entrent dans la formule par leur fonctionnement à intérêts composésjusqu'au terme de l'exploitation à un taux qui est précisément celui de cette exploitation.

Les produits des éclaircies peuvent être employés par le propriétaire en placements d'une autre nature; alor's, le taux non de la forêt, mais de cette entreprise mixte, peut augmenter beaucoup. 
Supposons que le propriétaire de la forêt qui nous sert d'exemple place le produit de l'éclaircie faite à 70 ans en valeurs mobilières, rapportant $41 / 20 / 0$, il aura tous les 100 ans.

Coupe principale.............. 4000"

Valeur de l'éclaircie grossie de ses intérêts à $41 / 20 / 0$ pendant $100-70=30$ ans soit $400 \times 3,745 \ldots \ldots \ldots \ldots \ldots . \frac{1498 \quad \cdots}{5498^{\mathrm{f}} \text { ) }}$

Cette spéculation lui procurera un placement de $4,420 / 0$ qu'on calculerait en plaçant le capital de $500 \mathrm{fr}$. en présence du produit $5,498 \mathrm{fr}$. par la formule

$$
\log .(1+x)=\frac{\log .5998-\log .500}{100}
$$

La forêt elle-même ne rapporte que 2,60 0/0 lorsque le produit des éclaircies est simplement employé aux besoins ordinaires du propriétaire.

Un auteur forestier n'a pas craint de donner cette étrange conception comme une manière de calculer le taux de placement des forêts éclaircies. Il a soutenu que ces coupes intermédiaires étaient capables de faire rapporter aux fulaies pleines un taux avantageux de $41 / 2$ à $50 / 0$. Or, qui ne voit au seul exposé du calcul que la forêt n'est pas placée à ce taux? A l'exploitation forestière, on substitue une entreprise mixte de forêt et de placement mobilier dont le résultat final est un taux avantageux. La forêt fournit le moyen d'opérer cette combinaison financière, mais voilà tout; ce n'est pas elle qui rapporte $4,420 / 0$.

Cette observation, destinée à réfuter une erreur commise montre, toutefois, que l'exploitation périodique avec éclaircie offre au propriétaire une ressource qui n'est pas sans avantage: l'exploitation périodique est surtout destinée à constituer un capital, c'est une caisse d'épargne fonctionnant seule avec la sécurité la plus grande. Les éclaircies fournissent le moyen d'attendre la constitution du capital; elles procurent aussi le moyen d'alimenter la caisse d'épargne ordinaire qui capitalisera à côté de la 
forêt, les sommes provenant de ces coupes et que le propriétaire y déposerait pour hâter la formation du capital. Les compagnies d'assurance qui cherchent des capitalisations à longues échéances ne paraissent pas connaître assez les ressources que les forêts peuvent leur offrir.

No 126. - Exploitations aménagées. - La formule du taux de placement des exploitations périodiques

$$
\mathrm{C}=\frac{\mathrm{R}+r(1+x)^{n-e}+r^{\prime}(1+x)^{n-e^{\prime}} \pm \frac{a}{x}}{(1+x)^{n}-1}
$$

est générale, en ce sens qu'elle fournit l'équation du taux de placement des forêts aménagées. Dans celles-ci, en effet, la périodicité de la coupe principale et des coupes d'éclaircie se réduit à un an : $n=1, e=1, e^{\prime}=1$ et $n-e=0$; la formule devient:

$$
\mathrm{C}=\frac{\mathrm{R}+r+r^{\prime} \pm a}{x}
$$

d'où on déduit :

$$
x=\frac{\mathrm{R}+r+r^{\prime} \pm a}{\mathrm{C}}
$$

Cette transformation, qui a pour mérite de montrer l'exactitude de la formule des exploitations périodiques, fournit la valeur du taux par une expression très simple et un calcul très facile puisqu'il suffit de diviser le revenu par le capital engagé.

Le taux de placement des exploitations aménagées s'exprime par le rapport du revenu annuel de toute nature (coupe principale, éclaircies, chasse, menus produits du sol) au capital engagé (sol et matériel). Le taux est net quand les charges ont été défalquées du revenu.

Exemples : $1^{\circ} 30$ hectares de taillis ordinaire aménagés à 30 ans produisent $980 \mathrm{fr}$. par an ; on estime le sol $420 \mathrm{fr}$. et l'ensouchement $122 \mathrm{fr}$.; on évalue que les bois de 1 à 30 ans qui garnissent le terrain ont une valeur de $9,000 \mathrm{fr}$. 
Le capital engagé est :

Terrain : $30 \times 420 \ldots \ldots \ldots \ldots \ldots$ 12600 "

Ensouchement : $30 \times 122 \ldots \ldots \ldots \ldots 3660$ »

Matériel-bois................. 9000 ,

Valeur de l'immeuble.... 25260

Le revenu étant de 980 francs, le taux brut de placement sera :

$$
\frac{980}{25260}=3.880 / 0
$$

$2^{\circ} 120$ hectares de futaie pleine, aménagée à 120 ans, produisent un revenu annuel de 6660 francs ainsi détaillé :

Coupe principale............ 6000

Eclaircie à 60 ans............... 100 »

- à 80 ans................... 300 »

- à 100 ans................ $\frac{500 \quad \text { ") }}{6900^{\mathrm{f}} \text { ” }}$

Revenu de la chasse et des produits an-

nuels...................... $\frac{360^{\prime \prime}}{7260^{\text {f }}}$

Frais de garde, d'administration et d'im-

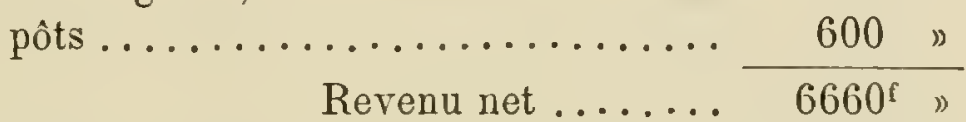

Le capital engagé a été évalué à 354,000 francs, savoir :

Sol $400^{f}$, semis $50^{f}$, soit $120 \times 450 \ldots 54000^{\text {f }}$ n

Natériel-bois de 1 à 120 ans ......... $\frac{300000 \text { ") }}{354000^{\text {" }}}$

Le taux net du placement est

$$
\frac{6660}{354000}=1,90 / 0
$$

$N^{\circ}$ 127. - Pour obtenir le taux de placement d'une forêt, il faut naturellement évaluer en argent le revenu et le capital engagé, celui-ci se composant du sol et du matériel d'exploitation.

Dans la pratique, il faudra donner à ces éléments non des valeurs de convention ou de calcul, mais des valeur's absolues et vénales, c'est-à-dire le prix qu'on en retirerait si on les metiait en vente. Ce principe n'a pas besoin d'être 
démontré; c'est la réalité des choses qu'il faut chercher et non la fiction.

Dans la conception théorique d'une exploitation, c'est-àdire quand on se propose de monter une exploitation à un âge déterminé et sur un sol de valeur connue, il faut, pour calculer le taux du placement, rechercher le revenu dont elle est susceptible et la valeur du matériel-bois dont il faudra garnir le terrain.

En ce qui concerne le revenu, il n'y aura aucune indécision, du moment où l'on connaît le mode de traitement cultural qui est toujours commandé par la pratique locale. Les détails que nous avons donnés sur la possibilité, c'està-dire sur l'expression pratique du revenu, serviront à indiquer la valeur du revenu correspondant au plan d'exploitation choisi; il n'y aura qu'à appliquer les prix moyens que l'exploitant est en droit d'espérer dans la localité.

$\mathrm{Si}$, par exemple, on projette de constituer une exploitation en taillis sous futaie à 30 ans avec 110 arbres par hectare (60 baliveaux, 40 modernes, 10 anciens), on saura toujours que le revenu sera une coupe de $1 / 30$ de la contenance avec 60 arbres d'abandon (20 modemes, 50 anciens, 10 bisanciens); il suffira d'appliquer à ces arbres les prix qui résultent de la proportion dans laquelle les essences existent dans la forêt.

En ce qui concerne le capital-bois, l'évaluation en est plus difficile: le revenu projeté indique bien la composition du matériel; ce sera par exemple 30 coupes de 1 à 30 ans avec les arbres dont le plan de balivage indique le nombre et la disposition ( $\mathrm{n}^{\circ} 83$ ).

Ce matériel sera, pour une futaie pleine, la succession des contenances égales peuplées de bois ayant la suite complète des âges depuis 1 an jusque l'âge d'exploitation.

Pour le jardinage lui-même, qui présente une si grande confusion d'éléments, il n’y a aucune témérité à assimiler 
son matériel à celui d'une futaie pleine d'égale contenance et dont toutes les tiges seraient mélangées entre elles sans aucun ordre. Du moins, cette assimilation, si elle n'est pas entièrement exacte, permet d'avoir une idée suffisante de l'importance du matériel.

Mais, si la connaissance de la constitution de la forêt permet d'analyser avec exactitude le matériel nécessaire à une exploitation déterminée, il devient plus difficile d'estimer en argent la valeur de ce matériel.

Il y a d'abord l'ensouchement dans les taillis et le semis initial dans les futaies, auxquels il faut assigner un prix: lorsque le semis ou la plantation sont artificiels, l'évaluation sera facile, car on sait généralement ce qu'il faut débourser pour semer, planter et assurer le succès clu travail. Le semis naturel s'évaluera sur' la même base, car s'il n’a exigé aucun déboursé, il a demandé du temps et il correspond à une privation de jouissance dont la valeur est analogue. L'évaluation de l'ensouchement est plus délicate, car on n'achète pas de souches pour les planter et on ne vend pas d'ensouchement vivant; il y a là une question d'appréciation dont la solution sera fournie par la comparaison de la valeur des sols voisins non ensouchés, par la dépense d'un bon semis ou d'une plantation réussie, et, enfin, par le tact expérimental de l'estimateur. Il en est ainsi dans toutes les évaluations : de ce côté, il n'y a pas encore grande difficulté.

Quant au matériel-bois, les arbres et les peuplements qui le composent seront estimés d'après leur volume à leurs différents âges et d'après les marchandises qu'on peut en retirer; mais il y a une difficulté considérable pour les bois et les peuplements en croissance, dont on ne connaît pas encore la marche de végétation, c'est-à-dire la loi suivant laquelle le volume et la valeur se forment avec l'âge. Quand les arbres et les peuplements ont acquis des dimensions utitisables, on obtiendra leur valeur par 
cornparaison avec des bois de même âge dans la localité et en terrain semblable. Mais quant aux bois trop jeunes, pour être vendables, c'est-à-dire pour avoir un prix assis et courant dans la localité, l'embarras sera grand. Notre avis est que le mieux est de les négliger, car on ne saurait estimer ce qui n'a pas de prix et ils entrent d'ailleurs en trop faible importance dans les exploitations à long terme pour qu'il y ait lieu de s'en occuper. C'est une quantité très souvent négligeable et on peut d'ailleurs toujours lui assigner une valeur d'appréciation qui sera sans grande influence pour le calcul du taux de placement.

Il existe cependant certaines forêts exploitées à très court terme dans lesquelles les jeunes bois non vendables ont cependant une certaine importance; ainsi des taillis à écorces de chêne sont exploités dans les Ardennes et dans le Luxembourg à 12 ou 15 ans; on peut assigner un prix à la coupe exploitable et à quelques-unes qui la précèdent; mais les jeunes taillis n'ont aucune valeur d'utilisation et un marchand de bois n'en donnerait aucun prix; elles ont cependant une valeur incontestable pour le propriétaire et il faudra bien leur appliquer un prix quand il s'agira d'évaluer le matériel d'exploitation, soit pour estimer la forêt, soit pour calculer le taux du placement.

Cette question de la valeur à donner aux jeunes bois qui n'ont pas encore acquis leur valeur commerciale est une de celles qui ont le plus occupé l'attention des torestiers. Nous verrons par quel procédé on peut arriver à leur assigner une valeur approchée et convenablement raisonnée ( $n^{\circ} 143$ ). Nous verrons également par quel procédé on arrive à calculer le taux de placement d'une exploitation aménagée sans connaître la loi de formation du matérielbois, sans, dès lors, en déterminer la valeur et sans s'écarter des limites d'une approximation suffisante ( $\left.n^{\circ} 131\right)$.

No 128. - Nous avons dit que le sol devait être estimé 
au prix où on le vendrait dans la localité; on arrivera à la connaissance de ce prix en s'éclairant par les ventes de terres d'égale qualité placées dans le voisinage de la forêt. En général, les terres en friches valent moins que celles de la forêt, parce que l'humus de celles-ci y a créé de longue date une fertilité qui a son prix; les terres cultivées aux rives de la forêt ont une valeur à peu près égale, car la modeste couche arable de ces cultures équivaut à la qualité de terre à bois du sol forestier.

C'est une affaire d'appréciation; toutes les estimations y sont soumises.

L'évaluation pourra souvent être éclairée par des ventes de forêts faites dans la localité : là, par exemple, une forêt de 10 hectares a été vendue $12,500 \mathrm{fr}$. ; si la visite de la forêt permet d'évaluer la superficie boisée à 7,500 fr., c’est que le sol a été compté 5,000 fr., ou 500 fr. l'hectare, ensouchement compris.

Le principe est d'estimer le sol au prix qu'on en obtiendrait si on le vendait rasé et désouché. On conviendra que le bois toujours mobilisable qui existe sur un terrain n'entre pour rien dans sa valeur ; la loi de l'offre et de la demande forme seule le prix des choses. C'est dire que, dans l'estimation du sol des grandes forêts, on devra faire ce qu'on appelle la déduction du gios, c'est-à-dire diminuer le prix de l'hectare en raison de l'abondance de la marchandise mise en vente.

Lorsqu'on plante une forêt, il faut bien compter le sol pour sa valeur d'achat. Pourquoi en serait-il autrement pour une forêt créée et naturelle, et pourquoi ne prendrait-on pas pour prix du terrain la somme qu'on en retirerait si on le vendait?

$\mathrm{N}^{\circ}$ 129. - Ce procédé si simple d'estimation des sols forestier's a été cependant l'objet de vives critiques et on a cherché à exprimer la valeur des sols boisés par un 
mécanisme de calcul et par des complications scientifiques de nature à faire plus honneur à leur inventeur qu'à la vérité.

On a posé en principe qu'un sol n'a de valeur que par les produits qu'on peut en retirer, principe spécieux et même faux, car un champ qui produit 15 hectolitres de blé à l'hectare est capable d'en rapporter 25 par des engrais convenables sans que la valeur du terrain change; c'est l'offre et la demande qui seules forment les prix. Sans doute, la demande se porte, en général, davantage vers ce qui est susceptible de produit avantageux, mais c'est à la condition qu'il y ait assez d'acquéreurs pour créer un surcroît de concurrence : combien de terres susceptibles de plantations et de revenus forestiers élevés ont cependant très peu de valeur dans certaines localités!

Voici de quelle manière on a fait usage de la formule génératrice d'un revenu périodique pour évaluer les sols de forêts :

$$
\mathrm{Sol}=\frac{\mathrm{R}}{(1+t)^{n}-1}
$$

En admettant, par exemple, un taux moyen pour l'emploi de l'argent dans une localité, soit $30 / 0$, on évalue à $496 \mathrm{fr}$. le sol d'une forêt qui rapporte $400 \mathrm{fr}$. en 20 ans :

$$
400 \times 1,240=496 \mathrm{fr} .
$$

Il est facile de voir combien ce procédé est inexact.

D'abord, la valeur fournie par cette expression ne représente pas le sol, mais bien le capital générateur du revenu, c'est-à-dire le terrain garni de son matérielbois, ensouchement, semis et même les arbres du balivage.

Ensuite, ce procédé n'est jamais pratiqué entre vendeurs et acheteurs : s'il arrive parfois qu'on multiplie le revenu d'un champ par le facteur $\frac{1}{t}$ pour obtenir sa valeur capitale, on ne procède jamais ainsi que pour des produits 
annuels. En étendant ce procédé par voie d'analogie mathématique, et en se servant d'une formule compliquée, on s'expose à employer une mesure qui n'est jamais usitée et à obtenir seulement par hasard la valeur des chöses mises en vente.

En outre, en supposant que l'estimateur défalque du résultat la valeur du matériel-bois, il reste en présence de deux appréciations délicates et presque insolubles : quel taux choisir et quel âge adopter pour les introduire dans la formule? On conviendra qu'il est bien plus simple et plus pratique de substituer à ces deux éléments si délicats une simple appréciation portant en bloc sur la valeur du terrain.

Il convient, en effet, de remarquer que, pour un même taux, la valeur d'un même terrain deviendrait très variable par l'emploi de ce procédé, selon l'âge qui aurait été choisi.

Voici le résultat de ce calcul effectué à $30 / 0$ pour des produits recueillis à différents âges :
A 20 ans ..... 400 $400^{\mathrm{f}}$ "
Sol $=496^{\mathrm{f}}$
A 50 ans .....22000
$\mathrm{Sol}=590$ "
A 100 ans ...... 4000 ,
Sol $=219 \quad "$
A 150 ans ...... 8000 》
$\mathrm{Sol}=96$

De sorte que, dans la même localité, le même sol, implanté des mêmes essences, aurait des valeurs variant de $96 \mathrm{fr}$. à $590 \mathrm{fr}$. l'hectare, uniquement à raison de l'âge où il peut plaire à un propriétaire de couper son bois.

On s'est livré, dès lors, à des considérations à perte de vue sur le taux qui doit être employé : taux moyen des placements en immeubles ruraux, taux d'escompte à la Banque de France, taux des prêts mobiliers à long terme, etc., comme si la mesure du loyer des capitaux n'était pas variable avec la nature du capital et les conditions économiques de la localité.

On a plus erré encore quand il s'est agi de déterminer quel âge il fallait choisir pour l'introduire dans la for- 
mule. On a dû inventer un âge d'exploitation qui serait le plus avantageux au point de vue du taux de placement et imaginer une exploitabilité commerciale des plus nébuleuses. Nous nous sommes assez expliqué sur cette singulière conception pour n'avoir pas à y revenir ( $\left.{ }^{(}\right)$.

Tout esprit impartial conviendra que la valeur d'un terrain ne peut varier ainsi avec l'âge auquel un propriétaire veut exploiter sa forêt, et que le seul procédé simple et légitime d'en obtenir le prix-courant, consiste à s'éclairer par les ventes de terrain faites dans le voisinage de la forêt.

$N^{\circ} 130$. - Il est utile de se rendre compte de l'effet produit sur le taux de placement d'une forêt par l'amélioration de l'outillage général et par le capital consacré à son exploitation.

L'effet est si simple qu'un exemple suffit à le montrer.

Une forèt de 30 hectares de taillis ordinaire aménagée à 30 ans et estimée 25,260 fr. rapporte tous les ans $980 \mathrm{fr}$.; le taux du placement de cet immeuble est ainsi de 3,88 0/0 $\left(\mathrm{n}^{\circ} 126\right)$.

On empierre certaines laies de coupes de manière à les adapter à la vidange; on dejpense pour cet objet $3,000 \mathrm{fr}$. et on espère une plus-value de $1 \mathrm{fr}$. par mètre cube, soit une amélioration de $150 \mathrm{fr}$. sur le revenu annuel. L'immeuble revient ainsi au propriétaire à $28,260 \mathrm{fr}$. et le revenu est porté à $1,130 \mathrm{fr}$. Le taux de placement est dès lors de $40 / 0$.

$$
\frac{1130}{28260}=40 / 0
$$

Le propriétaire qui améliore ainsi sa forêt a bien sa dépense placée à 50,0 . puisqu'il en recueille $150 \mathrm{fr}$.; mais c'est un placement dont le capital n'est plus disponible et qui s'est incorporé au sol ; c'est le placement général de l'immeuble qui doitêtre examiné. A ceux qui hésiteraient à

(1) Estimations concernant la propriété forestière, Note p. 249. 
améliorer ainsi leur forêt et qui tiendraient pour minime l'avantage d'un placement passant de $3,88 \quad 0 / 0$ à $40 / 0$, nous ferons observer que la négligence peut parfois se payer fort cher.

Une forêt dont l'accès est difficile est bientôt dédaignée par les marchands de bois, si la viabilité des torêts voisines vient à s'améliorer: l'immeuble nég'ligé perd à la fois en capital et en revenu, et le placement cesse d'être avantageux.

No 131. - Le taux de placement est-il le même pour les exploitations aménagées et périodiques faites au même âge? En d'autres termes, deux exploitations de même nature culturale, réglées au même âge, assises en même terrain et ayant les mêmes essences, ont-elles leurs fonds engagés placés au même taux, qu'elles soient périodiques ou aménagées?

L'affirmative semble résulter de la formule générale $\left(\mathrm{n}^{\circ} 126\right)$ dans laquelle il suffit de ramener à une année la périodicité de la coupe principale et des coupes d'éclaircies pour obtenir la formule du taux de placement des exploitations aménagées.

$$
x=\frac{\mathrm{R}}{\mathrm{C}}
$$

Cependant, quand nous avons cherché le taux de placement d'une exploitation périodique en taillis ordinaire à 30 ans, produisant $980 \mathrm{fr}$. avec un capital engagé cle $542 \mathrm{fr}$., nous avons trouvé $31 / 20 / 0$ ( $\left.n^{\circ} 122\right)$. Ensuite, quand nous avons calculé le taux de placement de la même exploitation aménagée, nous avons constaté que le taux était de $3,880 / 0\left(n^{\circ} 126\right)$. Cette différence tient uniquement à ce que le matériel-bois de l'exploitation aménagée a été estimé $9,000 \mathrm{fr}$. On a donné aux coupes en croissance une valeur trop faible et le taux s'est amélioré.

Le taux de placement n'est donc égal de part et d'autre que si on évalue les bois en croissance suivant la loi des 
intérêts composés, c'est-à-dire que si on admet que les valeurs créées par la végétation ont une marche ascendante identique à celles des intérêts composés au taux même du placement de l'exploitation périodique.

En effet, une forêt aménagée à $n$ ans est composée de $n$ exploitations périodiques à $n$ ans. Si on donne à chaque coupe sa valeur au taux $t$ suivant la règle d'estimation ( $\left.\mathrm{n}^{\circ} 51\right)$, on peut représenter la valeur de la forêt aménagée par

$\mathrm{C}=\mathrm{R}\left(\frac{1}{(1+t)^{n}-1}+\frac{(1+t)}{(1+t)^{n}-1}+\frac{(1+t)^{2}}{(1+t)^{n}-1}+\ldots \frac{(1+t)^{n}}{(1+t)^{n}-1}\right)$

La partie entre parenthèses est une progression géométrique à raison $1+t$ dont la somme des termes est fournie par la r’ègle:

ce qui donne:

$$
\alpha \frac{\left(q^{n}-1\right)}{q-1}
$$

$$
\mathrm{C}=\mathrm{R} \times \frac{1}{t}
$$

Le taux $t$ est donc le même quand la suite des peuplements en croissance est estimée comme une progression d'intérêts du fonds générateur composés au mème taux $t$.

On voit ainsi que l'égalité du taux de placement n'existe qu'en faisant une hypothèse sur la valeur du matériel-bois de l'exploitation aménagée.

Or, nous verrons que cette hypothèse est très inexacte; il faudra donc toujours estimer directement ce matériel d'après les marchandises qu'on peut en retirer, le taux dût-il être différent de celui de l'exploitation périodique faite au même âge. C'est la réalité qu'on doit chercher et non un taux fictif fondé sur une supposition.

Cependant, quand on projette un aménagement et quand on veut se rendre compte du taux auquel seront placés les fonds engagés, on pourra, à défaut de forêt similaire servant de comparaison, adopter pour base du calcul celui de l'exploitation périodique faite au même âge.

Exemple: Le propriétaire d'une forêt de 30 hectares de taillis so us futaie, aménagée à 30 ans veut porter son aménagement à 
40 ans, âge auquel il espère un produit de $1,500 \mathrm{fr}$. par hectare, soit pour la coupe de $0^{\text {h }}, 75$, un revenu de $1,125 \mathrm{fr}$.; le sol vaut arec l'ensouchemeut $660 \mathrm{fr}$. Le propriétaire se demande quel taux de placement il obtiendra pour sa propriété ainsi modifiée. La réponse approximative est dans l'équation :

$$
\begin{aligned}
\log \cdot(1+x) & =\frac{\log \cdot 2160-\log \cdot 660}{40} \\
x & =30 / 0
\end{aligned}
$$

Un pareil calcul ne fournit qu'un simple renseignement fondé sur une hypothèse faite sur la valeur de matériel à créer; mais comme cette hypothèse n'est pas toujours éloignée de la réalité des choses, on obtient ainsi une indication utile pour éclairer l'exploitant et un procédé de calcul rapide arec une approximation souvent très suffisante.

No 132. - La règle précédente, en vertu de laquelle le taux de placement d'une exploitation forestière se déduit de celui de l'exploitation périodique, sert à déterminer la loi suivant laquelle les capitaux sont placés dans les forêts.

Pour un terrain de même valeur et peuplé des mêmes essences, le taux de placement augmente avec l'âge, passe par un maximum et décroit ensuite, de telle soite que l'abaissement du taux est la note caractéristique des exploitations intensives.

Sur un hectare estimé $500 \mathrm{fr}$. arec son semis initial, une forèt mélangèe de hètres et d'épicéas fournit les produits et les taux de placement ci-après :
A 20 ans...... $400^{\mathrm{f}}$ D $\quad$ taux $2,040 / 0$
A 50 ans...... $2000 »-3,630 / 0$
A 100 ans...... $4000 » \quad-2,420 / 0$
A 150 ans..... $8000 \gg-1,900 / 0$

Un taillis ordinaire valant avec l'ensouchement $700 \mathrm{fr}$. l'hectare offre les produits et les taux suivants:
A 20 ans...... $400^{\text {r }} 》 \quad$ taux $2,220 / 0$
A 25 ans...... 600 » $\quad-2,490 / 0$
A 30 ans...... 925 " $\quad-2,820 / 0$
A 35 ans...... 1200 $1 \quad-2,870 / 0$
A 40 ans...... 1450 D $\quad-2,750 / 0$ 
Il existe des forêts exploitées à plus de 200 ans dont le taux de placement ne dépasse pas $10 / 0$. Cela tient à ce que la valeur forestière créée par l'âge ne croît pas assez vite pour maintenir le chiffre du taux et pour faire contrepoids à la puissance d'accumulation de l'intérêt composé.

Dans les exploitations aménagées, le revenu s'accroît avec l'âg'e ( $\left.n^{\circ} 115\right)$ mais le matériel augmente dans une plus forte proportion de telle sorte que ce revenu reste faible relativement au capital engagé, ce qui fait naturellement baisser le taux du placement.

$\mathrm{N}^{\circ}$ 133. - Il arrive heureusement que, dans les exploitations à long terme, le produit des éclaircies augmente un pen le revenu et fait hausser le taux du placement. Il arrive, aussi, que les grandes forêts exploitées à âge avancé sont habituellement situées en des régions où le sol a peu de valeur, ce qui a pour effet d'accroître également le taux du placement.

Ce double correctif est-il de nature à rendre à peu près uniforme le taux de placement des forêts? Certains forestiers très estimables l'ont prétendu et ont même soutenu que ce taux était, en France, en moyenne de $30 / 0$ pour les placements en forêts, quel que soit l'âge de leur exploitation. Cette opinion s'est même si bien accréditée que la plupart des évaluations ont été faites sur la base de $30 / 0$ dans les estimations les plus importantes.

Nous ne pouvons partager cette manière de voir trop absolue et trop contraire à la manière dont sont constituées les exploitations de forêts.

Nous avons vu, en effet, que les éclaircies n'augmentent le taux du placement que dans une mesure très faible ( $\left.n^{\circ} 125\right)$. Il n'est pas exact non plus de croire que les terrains ont peu de valeur dans les régions forestières. La terre agricole y acquiert souvent le plus haut prix à 
cause de sa rareté et l'industrie pastorale sait tirer grand profit des terres incultes sans intervention de la coûteuse main-d'œuvre. Dans les plaines de France, de nombreux terrains délaissés par le labour s'offrent aujourd'hui au reboisement à des prix très inférieurs à ceux des pays de montagnes.

On s'est trop laissé dominer par la considération de l'exploitation périodique pour en déduire le taux du placement des forêts qui sont habituellement aménagées. On a admis que celles-ci avaient, à âge et terrain égal, le même taux de placement; cela suppose un matériel-bois de valeur égale à celui que produirait la loi des intérêts composés. Or, cette hypothèse est inférieure à la réalité $\left(\mathrm{n}^{0} 143\right)$ et le matériel-bois des exploitations aménagées est toujours plus considérable que les valeurs produites par les intérêts composés. Il en résulte que le taux du placement s'abaisse notablement dans les exploitations aménagcées à long terme à cause de l'importance du matériel engagé.

D'ailleurs, les forestiers qui ont soutenu cette thèse de l'uniformité dans le taux de placement des forêts, se sont mis en contradiction avec eux-mêmes, car ils ont justement démontré que les fortunes privées étaient, en général, incapables de posséder des forêts de haute futaie exploitées à long terme, à raison de la faiblesse de leur taux de placement.

Ils ont vu, non moins justement, dans ce fait, la raison économique des aménagements des forêts domaniales que l'État peut seul conduire aux âges les plus avancés dans l'intérêt du travail national. Si les forêts rapportaient uniformément $30 / 0$, la fortune des particuliers pourrait s'y placer, aussi bien et aussi g'énéralement, que dans les exploitations agricoles.

Nous avons la conviction, qu'il sera du reste facile à tous de vérifier, que les taux des placements en forêts sont 
extrêmement variables, comme ceux de toutes les exploitations du sol, et que pour une même valeur de terrain le taux s'abaisse graduellement à mesure que l'âge d'exploitation augmente.

$N^{\circ} 134$. - Les considérations précédentes permettent de résoudre deux problèmes importants sur l'âge d'exploitation :

$1^{\circ} \mathrm{A}$ quel âge faut-il régler la coupe d'une forêt pour avoir un taux de placement déterminé?

Puisque le taux de placement s'abaisse graduellement pour un terrain de valeur donnée, à mesure que l'âge de coupe augmente, on pourra saisir un moment où le taux de placement correspondra à celui qui est désiré. Il sera facile de recueillir dans la localité les valeurs des coupes à différents âges et de faire le calcul par l'équation.

$$
\log \cdot(1+x)=\frac{\log \cdot \mathrm{R}+\mathrm{C}-\log \cdot \mathrm{C}}{n}
$$

On pourra même, pour plus d'exactitude, faire les essais par l'équation générale donnée $n^{\circ} 124$. On verra alors si dans l'échelle des taux ainsi calculés, on rencontre celui qu'on s'est assigné pour but de l'exploitation.

Naturelleraent l'échelle de ces taux ne sera pas très étendue et il faudra que le propriétaire sache se contenter de celui qu'il est habituel de demander à un placement de tout repos, comme celui des forêts. Il faudra même qu'il sache apprécier que la forêt ne comporte ni réparation, ni perte de fermag'e et qu'il se contente d'un taux de faible apparence, mais de réalité toujours avantageuse, parce qu'il est toujours net et dégagé de toute éventualité ( $\left.n^{\circ} 4\right)$.

$N^{\circ}$ 135. - $2^{\circ}$ A quel âge faut-il exploiter une forêt pour obtenir le taux maximum que les forêts sont capables de fournir dans la localité ? 
D'abord, existe-t-il un moment oì le taux de placement atteint son maximum pour un même sol, peuplé de mêmes essences?

Ceci a fait doute pour certains esprits qui n'ont pas craint d'avancer que l'âge le plus avantageux au point de vue du taux de placement était le plus faible possible $\left({ }^{1}\right)$. L'existence d'un maximum à un âge qui est soulvent même assez avancé ne saurait échapper à qui voudra bien se rendre compte de la constitution des exploitations forestières : dans le début, au moment où la coupe est de faible valeur, le prix du terrain a un effet prépondérant dans la formule de l'intérêt composé qui sert à calculer le taux; celui-ci est alors assez faible. Ensuite, la valeur de la coupe augmentant de plus en plus, l'influence du capital engagé s'efface graduellement et le maximum doit arriver au moment où la valeur du sol cesse de faire sentir son effet sur la capitalisation des intérêts composés.

On voit dans les exemples cités au n 133 que le taux maximum 3,630/0 se présente à 50 ans dans la forêt de hêtre et d'épicéa. Ce maximum arrive à 35 ans $(2,870 / 0)$ dans le taillis ordinaire avec un sol ensouché de $700 \mathrm{fr}$. Il serait plus vite obtenu et arriverait à 30 ans si la valeur du terrain s'abaissait à $200 \mathrm{fr}$. l'hectare.

$$
\mathrm{C}=200^{\mathrm{f}} \text { » } \mathrm{C}=700^{\mathrm{f}} \text { ” }
$$

A 20 ans.... $400^{\text {f }}$ 》 taux $5,630 / 0$ taux $2,220 / 0$

A 25 ans... 600 " $-5,690 / 0-2,490 / 0$

A 30 ans.... $925 \gg-5,920 / 0-2,820 / 0$

A 35 ans... $1200 \gg-5,700 / 0-2,870 / 0$

A 40 ans... 1450 " $-5,410 / 0-2,750 / 0$

Il est entendu, d'ailleurs, qu'il s'agit seulement ici d'exemples particuliers dont on ne saurait généraliser les résultats ni convertir ceux-ci en règle applicable à tous les cas.

(1) M. Noirot-Bonnet, Mamuel de l'estimateur des forêts, 2e édition, 1856. 
Pour résoudre le problème qui nous occupe. il suffira donc d'évaluer le capital engagé (sol et matériel) comme il a été dit aux nos 127 et 128 . On se renseignera clans les forêts similaires voisines sur les revenus qu'on peut obtenir à différents âges; on mettra ces revenus en présence du capital engagé par l'équation logarithmique qui fournit le taux; l'échelle des taux ainsi calculée indiquera à quel âge le maximum arrive.

On peut remarquer que l'équation du taux peut se mettre sous la forme.

$$
(1+x)^{n}=\frac{\mathrm{R}}{\mathrm{C}}+1
$$

On obtiendra ainsi un moyen de calculer les taux par le tarif I d'une manière assez approximative pour révéler le moment du maximum .

Divisea le revenu périodique par le capital engagé, ajoutez une unité au quotient et cherchez dans le tarif I, à l’âge du revenu, le facteur qui en approche le plus; ce facteur correspond au taux de placement; continuez cette opération pour les âges et les revenus successif's et vous obtiendrez l'âge qui correspond au taux maximum.

Si on applique ce calcul à la belle sapinière dont l'exemple a été donné $n^{0} 115$ et si on la suppose installée sur un sol de $200 \mathrm{fr}$. formant avec un semis évalué $100 \mathrm{fr}$. un capital engagé de $300 \mathrm{fr}^{2}$., on trouve:

A 20 ans $90^{\mathrm{f}}$ " : $300+1=1,300$ taux 1,50 environ.

A 40 ans 630 ": $300+1=3,100-30 / 0$

A 60 ans 2000 »: $300+1=7,666-3,400 / 0$

A 80 ans 4370 »: $300+1=15,560-3,500 / 0$

A 100 ans 7930 ": $300+1=27,430-3,250 / 0$

A 120 ans 11820 »: $300+1=40,400-3,100 / 0$

A 140 ans 15400 »: $300+1=51,130-2,750 / 0$

C'est dans les environs de 80 ans que le taux du placement est le plus avantageux, mais il convient de remarquer: $1^{\circ}$ que les valeur's de la sapinière à ces divers âges sont assez fortes et supposent une localité où les bois se vendent cher; $2^{\circ}$ que les éclaircies dont on n'a pas tenu compte ici, sont de nature à re- 
lever le taux et à permettre d'espérer le maximum à un âge plus avancé; $3^{\circ}$ par contre, ce calcul suppose que le matérielbois de l'exploitation aménagée suit la loi des intérèts composés, ce qui est une hypothèse au-dessous de la vérité. Ce capital a réellement une valeur plus grande, ce qui tend à abaisser le taux.

Il n'y a pas de formule donnant le maximum du taux $x$ parce que on ne connaît pas la loi suivant laquelle le revenu $R$ varie avec l'âge $n$.

La méthode qui a été enseignée depuis une vingtaine d'années, à l'effet de trouver l'âge auquel il faut exploiter pour obtenir le placement le plus avantageux, au point de vue du taux du placement, ne répond pas au problème qui nous occupe et fournit une solution nébuleuse au sujet de laquelle nous nous sommes assez expliqué pour n'avoir plus à y revenir $\left(^{1}\right)$.

$\mathrm{N}^{\circ} 136$. - La diminution du taux de placement à mesure que l'exploitation forestière devient plus intensive, c'està-dire à mesure que l'âge d'exploitation augmente, a des conséquences économiques générales et très dignes d'occuper l'attention de tous ceux qui ont souci de la prospérité nationale. Nous éclairerons la discussion par un exemple, car rien ne précise l'idée mieux que le chiffre à l'appui du fait :

Une forêt de 120 hectares peuplée d'essences feuillues, aménagée à 120 ans, livre tous les ans à la coupe un hectare en futaie de 120 ans, avec quelques produits intermédiaires ou d'éclaircies.

Le bilan de cette exploitation peut s'établir de la manière suivante :

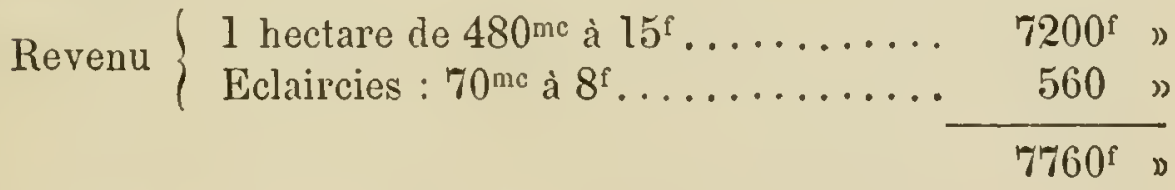

(1) Estimations concernant la propriété forestière, 1 vol. gr. in-80, 1886 , p. 2301 ; Note, p. 249. 
Matériel-bois évalué à.. 288000 »

Sol : $120^{\text {hoct }}$ à $300^{\text {f }} \ldots 36000$ »

Capital engagé. . 324000

Production............ $4^{\mathrm{mc}}, 500$

Rente........... $51^{\mathrm{f}} 60$

Taux du placement... $\quad 2,400 / 0$

Ce taux de 2,40 0/0 n'est certainement pas très élevé, mais une famille ayant de l'aisance, de la modération et de la sagesse dans la vie, a pu s'en contenter, heureuse de posséder un riche et bel immeuble et sachant que, toutes déductions faites, une ferme bâtie ne rapporterait pas davantage $\left(\mathrm{n}^{0} 4\right)$.

Mais voici une conséquence à tirer de la constitution mobilisable du capital d'exploitation des forêts et de la comparaison à faire avec le taux de placement des cultures forestières extensives.

Il y a dans la même localité, un grand nombre de taillis assis en sol de même valeur, exploités à 20 ans et produisant à cet âge 90 mètres cubes de menus bois à $5 \mathrm{fr}$. l'un, soit $450 \mathrm{fr}$. l'hectare de coupe.

Établissons le bilan d'une exploitation de 120 hectares dans ces conditions.

Revenu : 6 hectares à $450^{\mathrm{f}} \ldots \ldots \ldots \ldots \ldots \ldots 2700^{\mathrm{f}}$ 》

Matériel-bois évalué à.. $24000^{\mathrm{f}}$ 》

Sol............. 36000 »

Capital engagé.. $60000^{\mathrm{f}}$ " soit par hectare $500^{\mathrm{f}}$ » Production.......... $4^{\mathrm{mc}}, 500$

Rente........... $22^{\mathrm{f}} 50$

Taux du placement.... $41 / 20 / 0$

Voilà donc un immeuble forestier de faible valeur, qu'on peut avoir pour $60,000 \mathrm{fr}$., mais qui rapporte $4 \mathrm{l} / 20 / 0$ des fonds qu'on y mettra; quelle tentation pour le père de famille, si, par suite des exigences de la vie, son revenu devient insuffisant, ou si, ayant plusieurs enfants, il songe à ce que deviendra sa belle forêt partagée entre eux. Ne va-t-il pas faire le raisonnement suivant? 
Sur les $288,000 \mathrm{fr}$. de bois immobilisés dans la futaie, on réalisera en plusieurs années $264,000 \mathrm{fr}$., de manière à laisser les 120 hectares garnis de $24,000 \mathrm{fr}$. de jeunesses formant le matériel d'une exploitation de taillis, et avec les $264,000 \mathrm{fr}$. réalisés on achètera, à $500 \mathrm{fr}$. l'un, 528 hectares de taillis aménagés à 20 ans.

Et alors on possédera :

L'ancienne forèt 120 hectares ne valant plus que $60000^{\mathrm{f}}$ ” L'acquisition... $\frac{528-}{648 \text { hectares }}$ valant ......... $\frac{264000 \text { " }}{324000^{\mathrm{f}} \text {, }}$

Avec le même capital de $324,000 \mathrm{fr}$., on aurait en revenu:

Une coupe de 32 hect. 40 à $450 \mathrm{fr}$., soit $14,580 \mathrm{fr}$., près du double de revenu et cinq fois plus de terres avec la mème sécurité et la même nature de placement.

Il est vrai qu'avee 120 hectares on ne payait que $480 \mathrm{fr}$. d'impôts et de frais de garde ( $4 \mathrm{fr}$. par hectare) et qu'avec 648 hectares on paiera 2,592 fr. par an, nais qu'importe! La terre ne donne-t-elle pas dans la localité un crédit et une assiette qui rachètent ce revers de médaille? Et, tout compte fait, l'ancien revenu net, 7,280 fr., n'est-il pas encore amélioré de $4,710 \mathrm{fr}$.?

Quel est le propriétaire qui hésiterait ?

Il est certain aussi que si, avant de faire une transformation si tentante, le propriétaire consultait un forestier, celui-ci lui dirait que la conversion de la futaie en taillis n'a pas toutes les chances pour elle: l'ensouchement qui fait le taillis et en assure la durée n'est pas constitué dans la futaie; pendant de longues années la forêt convertie sera assez misérable. D'un autre côté, il arrivera un moment où par le seul fait de l'augmentation de toutes les choses nécessaires à la vie, le revenu de la futaie augmentera notablement, il ne faut pour cela qu'un peu de patience. Calculez, en effet, que chaque année le budget du pays augmente en moyenne de $50 / 0$, ce qui fait hausser le prix des objets nécessaires à la vie et qu'en même temps la quantité de métal précieux servant de numéraire augmente toujours, ce qui agit dans le même 
sens. Il est donc très probable que, en $20 \mathrm{ans}$, temps qui est nécessaire pour réaliser la superficie de la futaie et constituer le nouveau matériel d’âges gradués du taillis, ses produits auront peut-être doublé de valeur; tandis que les menus bois du taillis, toujours très abondants, ne suivront pas la même progression.

Certains propriétaires réfléchiront devant ces considérations et hésiteront à entreprendre la conversion, mais le plus grand nombre, moins sages, moins soucieux de l'avenir ou plus pressés par les besoins présents, n’hésiteront pas.

Beaucoup de propriétaires seront conduits à faire de tels emprunts au capital de leur's forêts que l'âge d'exploitation s'en trouvera abaissé et que, dans les forêts feuillues, le taillis avec peu ou point d'arbres tendra à remplacer la futaie.

L'abaissement général du loyer de l'argent est favorable à la conservation des grands arbres et des exploitations à long terme. Mais l'effet de cette loi économique ne suffit pas à prémunir la société contre le danger que lui fait courir la mise des forêts aux mains des particuliers.

Ce danger est réel, car l'exploitation à court terme ne livre à la consommation publique que des produits d'une minime utilité. Les grands arbres seuls lui fournissent la matière de travail dont elle ne saurait se passer.

L'exemple ci-dessus en fournit en quelque sorte la démonstration. Car les 648 hectares provenant de la transformation en taillis continueront bien à fournir 2,916 mètres cubes de matière ligneuse valant 14,580 fr., mais quelle différence pour l'utilité du travail qui en résulterait! Traités en futaie, ils auraient livré à la société une coupe de 41,904 fr. chaque année, c'est-à-dire trois fois plus de valeur d'utilité et d'échange.

Or, en quoi ces considérations importent-elles aux par- 
ticuliers, uniquement préoccupés du placement de leur argent et des nécessités de leur existence?

Aussi, toutes les futaies possédées jadis par les grands propriétaires et par l'Église, qui ont été confisquées et vendues à la Révolution, aussi toutes les forêts de l'État que des aliénations successives ont fait entrer dans le commerce, ont-elles été ramenées, sauf des exceptions louables mais rares, à des forêts à petit capital. La Statistique forestière de 1878 constate, non sans tristesse, qu'elles ne rapportent en moyenne que $24 \mathrm{fr} .14$ par hectare et par an.

Remarquons, en outre, que le capital d'exploitation destiné à la production des bois de service une fois détruit ne se reconstitue pas comme celui d'une exploitation agricole ou comme l'outillage d'une usine. Avec de l'argent on remonte ceux-ci, en une seule année; à l'industrie forestière, il faut un temps très long, un temps presque toujours séculaire pour reproduire son capital.

$\mathrm{N}^{\circ}$ 137. - Nous avons donc le devoir d'examiner les mesures d'utilité publique destinées à combattre, dans notre pays, cet effet désastreux. Elles consistent dans certaines dispositions de la loi et dans le rôle des forêts de l’État.

Jadis l'ordonnance de 1669, due aux préoccupations patriotiques de Colbert, imposait aux particuliers propriétaires de forêts l'obligation de laisser à chaque coupe au moins 32 baliveaux par hectare de taillis et 20 arbres par hectare de futaie; elle leur défendait de couper ces réserves sur taillis avant 40 ans et ces réserves sur futaie avant 120 ans. La même ordonnance imposait aux communes propriétaires de bois l'obligation de laisser un quart de leur bois croître en futaie ès meilleurs fonds $\left({ }^{1}\right)$.

(') Ord. 1669, tit. XXVl, art. Ier, et tit. XXV, art. 2. 
Ces dispositions ont formé la loi des forêts françaises jusqu'en 1827 ; mais elles ont dû disparaître ( $\left.{ }^{1}\right)$, car tous les citoyens n'étant pas propriétaires de bois et toutes les communes n'en possédant pas $\left({ }^{2}\right)$, il y avait une injustice certaine à faire payer aux propriétaires de certains immeubles un impôt que n'auraient pas payé les autres. C'est, en effet, un impôt réel que l'obligation pour un propriétaire de placer une partie de son argent à un taux minime, tandis que les autres ont toute liberté d'employer leurs fonds comme ils veulent.

Il n'existe dans notre législation qu'une seule mesure d'encouragement pour l'éducation des arbres de futaie : la valeur que celle-ci donne au revenu du sol n'est pas comptée pour l'assiette de l'impôt foncier. Les textes relatifs à la fixation du coëfficient de répartition de cet impôt, c'est-à-dire du revenu imposable, déclarent en termes formels que le gouvernement doit encourager l'éducation des arbres de futaie $\left({ }^{3}\right)$. C'est un accord parfait avec les principes généraux de la loi du 3 frimaire an VII, sur

(1) Le Code forestier de 1827 laisse, enl effet, toute liberté aux propriétaires particuliers d'exploiter leurs forèts à l'âge qui leur convient. Si l'article 93 impose encore aux communes un quart de réserve, celui-ci a cliangé de caractère el ne constitue plus qu'une mesure de préroyance destinée à leurs besoins imprérus-et faite uniquement daus leur intérêt (comparez 0 . Pégl. 134 et 68).

(2) Sur 35,989 communes, 11,479 seulement possèdent des forèts, dont 8,157 sont soumises au régime forestier et 3,322 ne le sont pas.

$\left({ }^{3}\right)$ Recueil méthodique pour le cadastre, du 22 janvier 1811. - Art. 368 : La plus-ralue que les bois de haute futaie acquicrent sur les bois taillis étant accidentelle et pouvant cesser après la coupe, n'est, dès lors, pas susceptible d'un allirrement cadastral fixe et imnuable et ces bois doivent être conıpris dans les expertises et les matrices cadastrales sur le même pied que ceux qui se trourent en taillis dans la commune ou les communes roisines. Art. 370 : Si un taillis contient des arbres de haute futaie, on ne doit pas estimer la place que ces arbres occupent conme si elle était courerte de taillis, ınais évaluer comme si ces arbres u’étaient pas plus âgés et plus forts que les autres, l'intention du gouvernement étant de favoriser les propriétaires qui laissent croitre leur bois ou partie de leur bois en lutaie. 
l'impôt foncier : "Toutes les majorations accidentelles du " revenu peuvent disparaître par la négligence ou l'avi" dité des détenteurs de l'immeuble; elles ne se pro" duisent que par leur sagesse et leur épargne. La loi " fiscale ne voulant ni encourager la négligence, ni taxer " la vertu, ne songe pas plus à abaisser la base de l'impôt " au-dlessous du revenu ordinaire de la terre, qu’à " l'élever au-dessus de ce chiffre moyen. ") (Recueil méth. du cadastre, 1811).

Voilà une disposition fort sage que les propriétaires de bonnes forêts bien garnies d'arbres feront bien d'avoir présente à l'esprit quand viendront les renouvellements de cadastre dans leurs communes $\left({ }^{1}\right)$.

Nous ne connaissons pas d'autres fidveurs législatives à leur égard et nous ne citons que pour mémoire:

La loi du 18 juin 1859, qui permet au gouvernement de défendre le défrichement des bois de particuliers dans certaines conditions;

Les articles 113 et 116 de la loi du 3 frimaire an VII, qui prémunissent les planteurs de terrains contre toute augmentation d'impôt ou leur assurent un dégrèvement de trois quarts pendant 30 ans;

L'article 226 du Code forestier, qui exempte de tout impôt pendant 30 ans les reboisements faits sur le sommet ou le penchant des montagnes, sur les dunes et dans les landes.

Ces dispositions qui ont pour but, la première d'assurer dans une certaine mesure la conservation de l'état boisé, les deux autres la création de nouvelles forêts, sont étrangères à ce que nous avons dit sur le danger de la disparition des arbres de futaie.

No 138. - Rôle des forêts de l'Etat. - Non seu-

( $\left.{ }^{1}\right)$ Voir notre étude : "L'impòt foncier, détermination du revenu imposable " (Revue des eanx el foréts, juin 1882). 
lement les propriétaires de bois ont acquis la liberté de couper leurs arbres comme il leur convient, et les communes ont été affranchies de l'obligation d'avoir le quart de leur bois en futaie, mais, en outre, diverses autres dispositions légales protectrices des grands arbres ont disparu de nos institutions.

Le droit coutumier immobilisait les arbres de futaie et les attachait si bien au sol qu'ils en devenaient en quelque sorte partie intégrante, - qu'ils conservaient, même abattus, leur qualité d'immeuble; - qu'ils étaient soustraits à l'action destructive des usufruitiers, des usagers et de tous ceux qui ont une jouissance temporaire sur les immeubles. Aujourd'hui, la distinction entre les futaies et les taillis n'existe plus que pour marquer des différences dans le monopole des officiers ministériels, pour les ventes aux enchères publiques ( $\left.{ }^{1}\right)$. Si elle existe encore dans les règles de jouissance imposées à l'usufruitier, et si celui-ci ne peut, en principe, toucher à la futaie $\left({ }^{2}\right)$, la. législation, devenue positive comme la société elle-même, n'impose plus, en réalité, aux jouissances temporaires d'autre règle que celle de lá liberté des conventions.

Jadis, les administrateurs des foréts soumises au régime forestier ne pouvaient faire abattre aucun arbre de futaie, même dépérissant, sans une permission du chef de l'État $\left({ }^{3}\right)$. Aujourd'hui les règlements administratifs permettent aux Agents forestiers d'apprécier souverainement l'état des futaies sur taillis et d'en ordonner l'abatage au moment du balivage des coupes $\left({ }^{4}\right)$. Cette confiance est fort honorable, mais elle a enlevé aux agents du service forestier leur force de résistance contre les besoins du

(1) Loi du 22 pluviōse an VII et du ร̌ juin $185 \% 1$.

() C. civ., art. วั92.

${ }^{3}$ ) Ord. 1669, tit. $\mathrm{XV}$, art. 12.

( $)$ Ord. du 1er autit 1827, art. T0. Déc. minist. du 6 lérrier 1838. 
moment et exposé les arbres de futaie à bien des destructions ( $\left.{ }^{1}\right)$.

Toutes ces mesures protectrices des arbres de futaie ont disparu et devaient disparaître dans une société démocratique qui a pour caractère une immense expansion vers le travail et l'échange. En France, comme dans toutes les sociétés modernes, il n'y a donc que les êtres moraux impérissables qui soient assez riches et surtout assez vivaces pour consacrer leurs forêts à la production soutenue des bois de fortes dimensions, dont le travail national ne pourra jamais se passel', malgré l'emploi du fer dans les constructions.

Or, de ce côté, il y a aussi bien des changements: les communes ont acquis la liberté d'exploiter leurs bois, plus cans l'intérêt de la localité qu'en vue de l'utilité nationale $\left(^{2}\right)$. L'Église, qui a perdu le droit de posséder, ne consacre plus sa patiente économie à créer des grands arbres. Nos puissantes compagnies financières sont bien plus des sociétés de crédit et de travail que des associations d'épargne.

L'État reste donc seul capable, en France, de consacrer ses forêts à la production des grands bois, matière indispensable dont le temps et l'épargne sont les seuls ouvriers. Qu'il ménage donc des arbres, beaucoup d'arbres, dans ses taillis sous futaie, dans ses forêts jardinées, dans ses futaies pleines, partout enfin où il le pourra. Le mode de traitement importe peu; l'arbre d'un taillis sous futaie rend autant de servicesque celui d'une futaie pleine; il a même l'avantagge d'être acquis en moins de temps.

(1) Dans 1 106,232 lsectares de forêts domaniales à chène, traités en taillis solis futaie, la statistique forestière de 1878 constate qu'il n'y a que 13 arbres de futaie par hectare (10 modernes et 3 anciens).

( ${ }^{2}$ ) Code forestier, art. 90 ; - Ord. 1827, art. 134. 
Ajoutons que c'est pour lui une obligation impérieuse, un devoir social plus encore que légal ( $\left.{ }^{1}\right)$.

Nous avons la conviction que l'abondance de l'argent, l'abaissement de son loyer et la cherté des salaires qui ont déjà fait reboiser, depuis trente ans, tant de terres jadis consacrées à l'agriculture, amèneront un jour des propriétaires de forêts à conserver des arbres et à y trouver Jeur intérêt. Le but de ce livre est de le leur montrer. Mais en attendant que cet espoir se réalise, les résultats en sont à lointaine échéance; si, quant à présent, quelques propriétaires avisés respectent leurs grands arbres et trouvent leur compte à les garder, il ne faut pas raisonner sur des exceptions ni laisser le pays exposé à de telles éventualités.

$\mathrm{N}^{\circ}$ 139. - Supposez un instant que, par suite d'une vente générale, si souvent conseillée et toujours repoussée par le bon sens public, toutes les forêts de l'État aient passé aux mains des particuliers. Pour que ceux-ci aient intérêt, d'une manière générale, à conserver à la consommation les richesses accumulées qui constituent la cô̂teuse exploitation des futaies, il faudrait que le prixdes produits vint à s'élever d'une facon calamiteuse, que le mètre cube de bois de service se vendît aux environs de $300 \mathrm{fr}$. et encore le taux du placement ne serait jamais bien élevé, car le capital d'exploitation augmente de valeur à mesure que le prix du mètre cube augmente.

Une pareille situation ne pourrait subsister et les bois venus de l'étranger, faisant, sur nos marchés, concurrence aux bois indigènes, les particuliers auraient vite intérêt à cesser une exploitation peu rémunératrice. Les forêts de France, ramenées à la petite culture forestière, ne livreraient plus à la consommation, comme le prouve l'exemple

(1) Code for., art. 15; - Ord. 1827, art. 68. 
du passé, que des bois de menu chauffage et de petit service. Il faudrait avoir recours aux violences de la loi; il faudrait protéger le public par un maximum de prix et forcer les particuliers par des peines sévères à conserver leurs futaies, tout en continuant à recevoir les bois de l'étranger, situation pleine d'injustice qui ferait peser sur les uns un impôt inique; car, on les forcerait à vendre aux autres, à très bon marché, ce qui leur coûte très cher à produire.

Ou bien, il faudrait interdire le marché français aux bois étrangers, entourer la patrie d'une de ces murailles prohibitives que réprouvent les lois économiques du travail et qui arrêtent le développement des sociétés modernes.

Que l'on ne croie pas à l'existence de forêts vierges, inexploitées dans les pays étrangers, où le commerce des bois ira alimenter notre travail. Certains livres ne manquent jamais de parler de ces mines inépuisables à l'appui d'étranges et captieuses théories. Aucun ne cite l'emplacement de ces greniers d'abondance, et dans la vieille Europe, comme dans le Nouveau-Monde, le cri d'alarme commence à retentir. Eh bien, supposez qu'il existe en quelque heurenx pays des ressources forestières assez puissantes pour subvenir aux besoins incessants de nos chemins de fer, de notre marine, de nos vignes, de nos constructions et de tous les besoins de notre dévorante civilisation. Supposez également qu'à la suite de la vente de toutes les forêts de l'État, la propriété boisée soit réduite à ne fournir que des menus bois et des bois de feu. Est-ce que, dans ces conditions, les États assez heureux et assez sages pour conserver leur's chênes et leurs sapins ne pourront pas nous les faire payer très cher? Ne pourront-ils pas, en frappant leurs bois de droits de sortie, lever sur la France le plus désastreux des impôts? lui faire la guerre la plus terrible, la guerre an travail?

Voilà donc où pourrait nous conduire la vente geénérale des forêts de l'État : 
Avec la liberté de l'importation, la contrainte des propriétaires de forêts : un impôt inégal, inique, pesant sur les uns au profit des autres.

Avec la liberté générale d'importation, d'exportation et d'exploitation: la destruction fatale de nos arbres de futaie, la mise du travail français aux mains de l'étranger.

Avec la prohibition d'entrée des bois étrangers, une élévation effrayante dans le prix du bois d'œurre, calamiteuse pour notre travail, un impôt nouveau sur le consommateur, impôt d'autant plus dur à supporter qu'il est impossible d'en prévoir les conséquences et d'en mesurer les effets.

A l'État, le devoir de produire des grands arbres, production coûteuse, car elle demande beaucoup de temps et beaucoup d'épargnes. L'État impérissable peut seul l'entreprendre et il doit la faire sur toutes les forêts qu'il possède.

Il est temps de songer que les forêts de l'État sont destinées à produrre de grands bois et de gros arbres et qu'elles ne sont pas pour lui un placement d'argent. La Révolution a trouvé l'État riche de 2 millions et demi d'hectares de forêt; après moins d'un demi-siècle, le domaine est réduit à 967,120 hectares ; dans celles de seize départements, la rente des forêts est inférieure à $5 \mathrm{fr} .\left(^{1}\right)$. Il est temps de songer a l'avenir!

( ${ }^{1}$ La rente et le prix moyen du mètre cube mesurent exactement les services rendus par les diverses forêts à la consommation publique. Cette rente est, en moyenne, pour les lorêts françaises, de $29 \mathrm{fr} .34$; elle est de 40 fr. 23 pour les forèts domaniales et de 24 fr. 14 pour les forèts particulières. Le prix moyen du mètre cube est, en France, pour les forêts de toutes sortes, de $9 \mathrm{fr}$. 23 ; il s'élève à 12 fr. 69 pour les forêts domaniales (Statistique 1878). I.es services rendus au public par les forèts de l'État sont donc encore susceptibles d'amélioration, c'est à la condition de ne pas se montrer trop exigeant pour le budget des recettes, de supprimer le pâturage qui dévore les forêts du Midi et de doter de chemins d'accès celles des régions montagneuses. 
Article 2.

\section{Taux de formation.}

Nos 140. - Importance de la question.

141. - Représentation des valeur's par l'intérêt composé pour l'arbre isolé.

142. - Idem pour le peuplement d'un seul âge.

143. - Valeur des récoltes acquises relativement au propriétaire de la forèt.

144. - Application : denier d'aménagement.

145. - Autre application : estimation d'une forêt exploitée à très court terme.

146. - Emploi spécial de l'estimation des récoltes erga dominum en cas de dommages causés à la propriété forestière.

147. - Exemple du calcul pour un taillis sous futaie.

148. - Ventes de récoltes sur pied; leur estimation.

149. - Application à l'expropriation pour canse d'utilité publique; cas d'un taillis ordinaire.

$\mathrm{N}^{\circ}$ 140. - La loi de formation des valeurs pécuniaires par la croissance des arbres et des peuplements est encore inconnue, et même la loi du volume échappe à nos connaissances par le défaut d'expériences exactement suivies. Cette ignorance empêche de résoudre avec certitude un grand nombre de questions relatives à la théorie des exploitations forestières.

On concoit l'utilité qu'il y aurait à connaître la loi de formation des valeurs fournies par la végétation.

C'est l'exploitant qui, pour se décider à régler sa forêt à un âge plutôt qu'à un autre, voudrait connaître de quelle valeur en bois il devra garnir son exploitation aménagée: sachant le revenu à 30 ans, par exemple, il voudrait savoir de quel capital-bois il devra monter son exploitation aménagée pour avoir ce revenu d'une manière annuelle et constante.

C'est l'estimateur de forêts qui, se trourant en présence 
de peuplements jeunes et pleins d'avenir, ne sait quel prix leur assigner et voudrait décluire leur valeur de celle qu'ils auront à un âge plus avancé.

C'est l'expert d'une compagnie d'assurance qui ne sait quelle valeur donner à des coupes voisines d'âges et qui ne peut estimer celle de 21 ans, incendiée, au prix de celle de 22 ans, par exemple, celle-ci ne présentant aucune différence autre que l'âge avec celle de 21 ans.

On peut être certain que le forestier qui trouvera la loi de formation des valeurs rendra un signalé service à l'économie forestière et fera progresser cette science d'une manière notable.

No 141. - On a cherché à exprimer la loi des valeurs par le moyen de la formation des intérêts composés et on a appelé tanx de formation ou de régétation, la mesure de cette loi entre l'époque de la naissance et celle de la coupe. en supposant que les prix marchent comme l'intérêt composé à ce taux.

On estime, par exemple, qu'une plantation de chênes coủte $20 \mathrm{fr}$., par mille plants, et que le chêne de 180 ans vaut $132 \mathrm{fr}$.

$$
\begin{gathered}
0,02(1+x)^{180}=132 \\
x=50 / 0
\end{gathered}
$$

L'application du taux de $50 / 0$ à la valeur initiale du plant

\begin{tabular}{|c|c|c|}
\hline 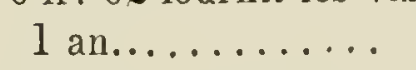 & $0^{\mathrm{f}} 021$ & 30 ans. \\
\hline 2 ans.. & 0022 & 60 ans... \\
\hline & () 023 & 90 ans. . . \\
\hline 4: & 0024 & 120 ans......... \\
\hline ns. & 0025 & 150 ans........ 30 \\
\hline ns. & 0033 & 180 ans....... 132 \\
\hline ans.......... & $\begin{array}{ll}0 & 0.53\end{array}$ & \\
\hline
\end{tabular}
$0 \mathrm{fr} .02$ fournit les valeurs intermédiaires suivantes :

Ce mode de calcul que nous avons vu employer par de nombreux experts ne représente en aucune facoin les valeurs successives dues à la végétation.

Voici, en effet, les prix de chènes sur taillis résultant d'un 
travail qui a porté, en 1875, sur un grand nombre d'arbres d'une forêt belge, limitrophe de France :

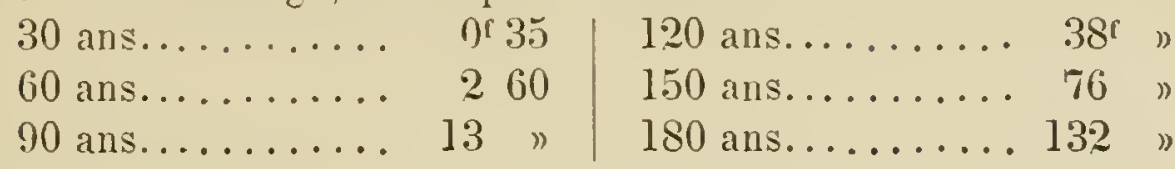

L'erreur de ce singulier calcul provient : $1^{\circ}$ de ce qu'il est très difficile d'assigner un prix au plant naissant; le taux varie dans des proportions énormes avec le prix aléatoire qu'on attribue aux jeunes plants ; $2^{\circ}$ la capitalisation, s'exerçant au début sur une valeur très faible, ne produit pendant longtemps que des prix insignifiants poul des arbres déjà âgés $(60,90,120$ ans). Cette capitalisation crôit ensuite très vite dès que les valeurs accumulées deviennent notables (150, 180 ans).

La figure 25 rend très apparente la différence qui existe entre les valeurs réelles et celles résultant d'un taux de formation ainsi calculé.

La vérité est que le taux de formation des valeurs dues à la végétation est variable à chaque âge de la vie.

Ainsi, en admettant que la tige naissante soit estimée 0,02 , le taux de régétation serait
De 1 à 30 ans ............ $9,400 / 0$
De 1 à 60 ans ........... $8,620 / 0$
De 1 à 90 ans ........... $7,150 / 0$
De 1 à 120 ans .......... 6,250/0
De 1 à 150 ans .......... 5, $150 / 0$
De 1 à 180 ans........... 5 „ $0 / 0$

En réalité, si on voulait assimiler les valeurs produites par la végétation à celles qui sont fournies par la loi de l'intérêt composé, cette assimilation ne serait exacte qu'ì la condition de faire variei le taux à chaque âge.

Ainsi, ces taux de formation seraient :
De 30 à 60 ans ........ $8,030 / 0$
De 60 à 90 ans.......... 6,670/0
De 90 à 120 ans......... 4,41 $0 / 0$
De 120 à 150 ans .......... 2,830/0
De 150 à 180 ans ......... 2,250/0 
Aucune loi mathématique ne saurait exprimer un pareil mode de formation.

$\mathrm{N}^{\circ}$ 142. - Il en est de même des peuplements : la conception du taux de végétation, pour représenter les valeurs en croissance, est contraire à la vérité.

Voici une sapinière dont les volumes, par hectare, ont été relevés sur les tables de M. Lorey, et qui a été estimée de la manière suivante :
A 20 ans........ $44^{\text {me }}$ évalués $180^{\text {r }}$ "
A 40 ans.......... 158 - 600 »
A 60 ans......... $333-2000$ »
A 80 ans.......... $547 \quad-4300$ ”
A 100 ans.......... $793 \quad 7900$ "
A 120 ans........ $985-11800$ „
A 140 ans......... 1105 - 15400 "

Le semis initial étant évalué à $65 \mathrm{fr}$., le taux de végétation pour la formation de 1 à 140 ans serait

$$
\begin{gathered}
65(1+x)^{1 \div 0}=15400 \\
x=40 / 0
\end{gathered}
$$

Si on représente les peuplements en croissance comme formés par les intérèts accumulés de $65 \mathrm{fr}$. à $40 / 0$, on obtient

\begin{tabular}{|c|c|c|c|c|}
\hline 20 ans & $141^{\mathrm{f}}$ & $"$ & 100 ans & $3282^{f}$ \\
\hline 40 ans .. & 312 & ” & 120 ans $\ldots \ldots$ & 7215 \\
\hline 60 ans . . & 682 & $"$ & 140 ans.... & 15400 \\
\hline 80 ans $\ldots \ldots \ldots$ & 1495 & " & & \\
\hline
\end{tabular}
par le tarif $I$, les valeurs suivantes :

La seule inspection de ces chiffres montre que les valeurs ainsi obtenues sont de pure fantaisie, la figure 26 l'indique graphiquement. Les motifs de l'erreur sont les mêmes que pour l'arbre isolé, et le taux de formation varie également à chaque âgge de la manière suivante :

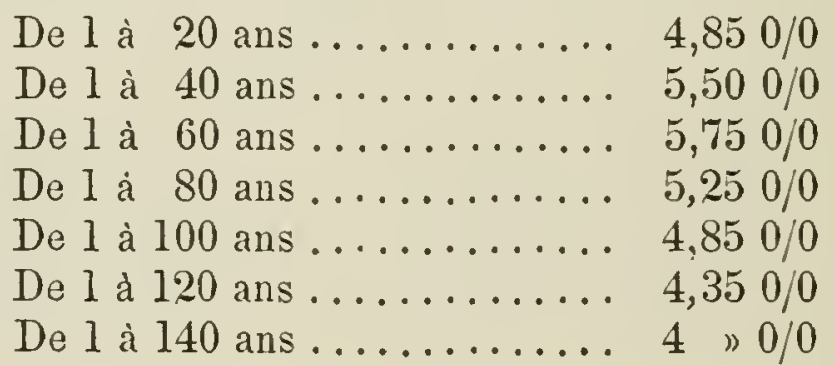


Si done on voulait assimiler les valeurs d'un peuplement en croissance à la formation par l'intérêt composé, il faudrait capitaliser à des taux variables, faibles au début, passant par un maximum et décroissant ensuite. Il en serait de même pour l'arbre; mais le maximum dans les taux rariables de la formation se rencontrerait à un âge peu avancé $\left(\mathrm{n}^{\circ} 141\right)$ ce qui le rend insaisissable. Nulle formule mathématique ne se prête à exprimer des valeurs ainsi formées par des taux variant à chaque âge.

$\mathrm{N}^{\circ}$ 143. - La question de savoir quel prix on doit assigner aux peuplements en croissance et notamment aux bois non encore vendables a, de tout temps, occupé l'attention des forestiers. On n'est pas encore arrivé à exprimer la loi de la valeur d'une manière absolue, mais on peut cependant la trouver d'une manière relative et sauf à n'en faire usage que dans les cas où cette valeur est vraie relativement à la personne qui a intérêt à l'invoquer.

Déjà, nous avons vu qu'on pouvait s'affranchir de la connaissance de cette loi quand il s'agit de calculer $a p$ proximativement le taux de placement d'une exploitation aménagée $\left(\mathrm{n}^{\circ} 131\right)$.

Il s'agit ici, non d'une approximation, mais d'un calcul donnant la valeur des récoltes acquises relativement an propriétaire de la forêt.

Indiquons d'abord de quelle manière le calcul s'effectue et nous montrerons ensuite la valeur des résultats ainsi que les circonstances dans lesquelles on peut convenablement s'en servir :

On calcule le taux du placement $x$ au moyen de la formule qui unit le capital $\mathrm{C}$ au revenu $\mathrm{R}$, en estimant directement $\mathrm{C}$ et $\mathrm{R}$ par l'analyse des conditions de l'exploitation.

$$
(1+x)^{n}=\frac{\mathrm{R}+\mathrm{C}}{\mathrm{C}}
$$


Ce taux $x$ étant déterminé, on obtient la valeur des récoltes en croissance en prenant l'intérêt du capital générateur $C$, calcul qui s'effectue très simplement par logarithmes ou par le tarif $I$ si le taux trouvé se rencontre dans ce tarif.

$$
C\left[(1+t)^{n}-1\right]
$$

En supposant que la sapinière du numéro précédent soit exploitée à 140 ans et établie sur un sol valant $435 \mathrm{fr}$. avec un semis initial de $65 \mathrm{fr}$. formant un capital engagé de $500 \mathrm{fr}$., le taux du placement est de $21 / 20 / 0$. Les valeurs successives des récoltes acquises sont, à ce taux:

\begin{tabular}{|c|c|c|c|}
\hline 20 ans & $319^{\mathrm{f}}$ & " & 100 ans .. \\
\hline 40 ans & 842 & ") & 120 ans . \\
\hline 60 ans & 1699 & $"$ & 140 ans . . . . . \\
\hline ans. & 3104 & ") & \\
\hline
\end{tabular}

Il suffit de considérer les valeurs d'estimation directe données au no 142 pour remarquer que celles de ce calcul leur sont inférieures, sauf dans le jeune âge. La figure 27, qui donne à la fois la courbe des volumes, celle des valeurs d'estimation et celle du calcul au taux de placement, montre cette différence. Toutefois, les prix réels ont-ils été exactement estimés? la discussion est toujours possible sur ce point délicat. On peut seulement affirmer:

$1^{\circ}$ Que cette méthode de calcul exprime mieux que toute autre la valeur des récoltes en croissance;

$2^{\circ}$ Que, cependant, elle parait donner des résultats inférieurs aux prix réels et on est certain qu'elle n'exprime pas la loi encore inconnue suivant laquelle la régétation forme les valeurs en croissance;

$3^{\circ}$ Mais ce calcul fournit exactement les valeurs pour lesquelles les peuplements en croissance entrent dans l'exploitation, c'est-à-dire que pour le propriétaire exploitant les coupes ont bien ce prix : le calcul dome ainsi exactement leur valeur relative erga dominum;

$4^{\circ}$ Enfin, il ne faut pas oublier que ce calcul n'exprime 
que les récoltes en croissance et non la superficie. Dans les exploitations dont la coupe se fait à blanc estoc, le récolte se confond avec la superficie, mais dans les exploitations à réserves, le plan de balivage doit s'ajouter à la récolte pendante pour exprimer la valeur de la superficie.

Nous pouvons maintenant apprécier le mérite du procédé ( $n^{\circ} 131$ ), qui consiste à déterminer le taux de placement d'une exploitation aménagée au moyen du taux de l'exploitation périodique faite au même âge. Les taux de placement des deux exploitations n'étant égaux que si le matériel-bois de l'exploitation aménagée a une valeur égale à celle qui résulte de la formation des intérêts composés au même taux, il en résulte que le procédé doit fournir un taux un peu trop fort, puisque la valeur du matériel-bois est, en réalité, plus considérable.

On conviendra que ce procédé est suffisant pour la conception théorique de l'exploitation, alors surtout qu'en cette matière les solutions cherchées ne sauraient jamais avoir l'exactitude absolue: quand on dit, par exemple, qu'une exploitation faite à 140 ans assure un placement de $21 / 20 / 0$, on doit toujours comprendre que le taux ainsi calculé est trop fort; il devrait être abaissé; mais les éclaircies étant de nature à le relever, cela peut faire compensation et rétablir la vérité. En tout cas, l'exploitant doit avoir la sagesse de se dire que c'est dans les environs de cet âge qu'il obtiendra un taux de placement de 2 1/2 0/0. En l'état actuel, l'économie forestière ne peut préciser davantage: on conviendra que cela suffit pour un renseignement de cette nature.

$N^{\circ} 144$. - Sous cette réserve, on peut calculer approximativement la valeur en argent du matériel d'une exploitation aménagée rapportant à son propriétaire un taux donné. On veut, par exemple, connaitre la valeur des bois d'î̀ges gradués qu'il faut toujours maintenir sur pied pour 
assurer une exploitation de 140 hectares, aménagée à 140 ans, produisant 15,400 fr. chaque année et constituant avec un capital engagé de $500 \mathrm{fr}$. par hectare, un placement à $21 / 20 / 0$.

On appelle taux d'aménagement la mesure du revenu par rapport au matériel-bois. Le denier d'aménagement est le facteur par lequel il faut multiplier le revenu pour avoir le matériel-bois; ce dernier est moindre naturellement que celui $\frac{1}{t}$ correspondant au capital engagé complet (sol et matériel); le facteur $d$ cherché est:

$$
d=\frac{1}{t}-\frac{n \mathrm{C}}{\mathrm{R}}
$$

Dans l'exemple précédent, le denier d'aménagement serait :

$$
\begin{gathered}
d=40-\frac{140 \times 500}{15400}=40-4,545 \\
d=35,455
\end{gathered}
$$

Le matériel nécessaire à un aménagement de 140 hectares est :

$$
15400 \times 35,455=546000^{\mathrm{f}}
$$

Comme vérification, la forèt aménagée rapportant $21 / 2 \quad 0 / 0$ aura une valeur approximative de :

$$
15400 \times 40=616000^{\mathrm{f}}
$$

ce qui fournit un taux de placement égal :

$$
\frac{15400}{616000}=2,500 / 0
$$

La démonstration de cette formule est très simple du moment où on admet que les récoltes pendantes ont des valeurs égales à la formation par intérêts composés au taux du placement de l'exploitation périodique. La suite des coupes vaut :

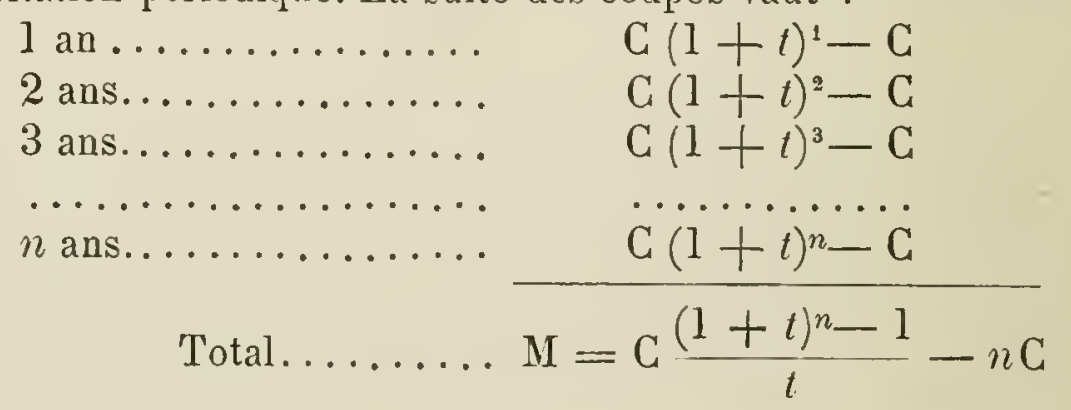



Si on remplace $C$ par sa valeur $\frac{\mathrm{R}}{(1+t)^{n-1}}$ le total des
récoltes pendantes $M$ est :

$$
\mathrm{M}=\frac{\mathrm{R}}{t}-n \mathrm{C}
$$

Cette formule fournit directement la valeur du matériel-bois.

Lorsqu'on vent un facteur $d$ pour multiplier le revenu, il suffit d'observer que

$$
\begin{gathered}
d=\frac{\mathrm{I}}{\mathrm{R}} \\
d=\frac{\mathrm{R}-n \mathrm{C} t}{t \mathrm{R}}=\frac{1}{t}-\frac{n \mathrm{C}}{\mathrm{R}}
\end{gathered}
$$

Il est à noter que cette expression ne fournit que la valeur des récoltes pendantes. C'est seulement pour les forêts à coupe de blanc estoc qu'elle représente là valeur de la superficie. Dans les forêts à arbres de réserve, la valeur du balivage initial est comprise dans $\mathrm{C}$; on devra ajouter $n$ fois ce balivage pour obtenir la superficie constituant le matériel-bois qui doit garnir constamment l'exploitation aménagée.

Exemple : Une forèt de 30 hectares de taillis sous futaie aménagée à 30 ans produit chaque amnée $1,110 \mathrm{fr}$.; le sol garni du matériel initial vaut $780 \mathrm{fr}$. (sol, $420 \mathrm{fr}$.; ensouchement, $122 \mathrm{fr}$. ; balivage, $238 \mathrm{fr}$.); il en résulte que le taux de placement de l'exploitation périodique est de $30 / 0$.

Le matériel-bois aura une valeur approximative de $20,740 \mathrm{fr}$. ainsi obtenue :

$$
\begin{aligned}
& 1110 \times 33,33 \ldots \ldots \ldots \ldots \ldots .37000^{\mathrm{f}} \text { " } \\
& -30 \times 780 \ldots \ldots \ldots \ldots \ldots \ldots . \ldots .23400 \text { " } \\
& +30 \times 238 \text {. Balivage ....... } 7140 \text { " } \\
& \text { Superficie........ } 20740^{\mathrm{f}}
\end{aligned}
$$

De pareils résultats ne sont que de simples renseignements destinés à éclairer l'exploitant et à l'avertir s'il venait à dépasser par ses coupes le revenu de sa forêt. Rien ne remplace dans la pratique l'estimation du matériel au moyen du cubage des peuplements et de la distribution du volume suivant les marchandises qu'on peut en retirer. 
$N^{\circ} 145$. - Lorsqu'on veut estimer une forêt pour en connaître la valeur vénale, il ne faudrait point employer cette méthode de calcul par le taux du placement pour assigner une valeur aux jeunes bois non encore vendables. Le commerce des biens compte, seulement, ce qui a prix de vente. Dans notre livre sur les Estimations concernant la propriété forestière, nous avons conseillé d'employer la méthode d'estimation absolue, dite des marchands de bois. Pour les forêts exploitées à un âge avancé, la valeur des jeunes peuplements non encore rendables a si peu d'importance qu'elle est une quantité véritablement négligeable, et jamais comptée par les acquéreurs. Dans les forêts moins riches en arbres vendables, les jeunes peuplements s'estiment par une appréciation directe qui a tout autant de mérite que les valeurs résultant de calculs fondés sur des hypothèses. Admettre que les bois en croissance suivent dans leur valeur la loi de l'intérêt composé, c'est faire une hypothèse sur la marche de la végétation; c'est en faire une autre sur le taux de placement : autant vaut remplacer un pareil arbitraire par une évaluation directe.

Mais voici un cas ou ce procédé pourrait conduire à une valeur d'estimation suffisamment approchée de la réalité; c'est celui des forêts exploitées à très court terme, dans lesquelles la coupe mûre a, presque seule, une valeur marchande, les autres étant au-dessous de l'âge où les bois commencent à se vendre utilement (certains taillis à écorces du Luxembourg et des Ardennes).

Un taillis dont le sol ensouché vaut 500 francs, produit à 15 ans 600 francs par hectare, le taux du placement de l'exploitation périodique est de $5 \mathrm{fr} .400 / 0$.

Une exploitation de 15 hectares aménagée à 15 ans vaudrait ainsi 11,100 francs et aurait une superficie de 3,600 francs.

$$
\begin{aligned}
& 600 \times \frac{1}{5,40}=600 \times 18,50 \ldots . \quad 11100^{\mathrm{f}}, \\
& \text { Sol : } 15 \times 500 \ldots \ldots \ldots \ldots \ldots .6 \frac{7500 "}{3600^{\mathrm{r}} "}
\end{aligned}
$$


Un canton de 22 hectares âgé de 6 ans à vendre dans une forêt de cette nature serait estimé

$$
22 \times 600 \frac{(1,054)^{6}}{(1,054)^{15}-1}=22 \times 685=15070
$$

Un pareil calcul ne fournira jamais qu'une estimation relatire faite en vue d'un acheteur désireux d'obtenir emploi de ses fonds à $5 \mathrm{fr} .400 / 0$ et disposé à maintenir l'exploitation à 15 ans.

Cette considération servira de guide dans l'usage qu'on voudra faire de ce mode d'estimation.

$\mathrm{N}^{\circ}$ 146. - Dans certaines circonstances, le calcul de la valeur erga dominum des récoltes acquises doit être utilisé à l'exclusion de tout autre procédé d'estimation : c'est quand il s'agit d'indemniser le propriétaire d'un dommage causé à la forêt et de réparer en argent la perte subie par l'exploitation; un incendie a détruit une coupe en totalité ou en partie, c'est un organe de l'exploitation qu'il faut remplacer par une valeur pécuniaire équivalente.

Le plus habituellement les parties s'entendent sur la valeur vénale à assigner à la récolte perdue, mais la discussion naît toujours à l'occasion des jeunes coupes auxquelles on ne sait quel prix attribuer.

Qu'il s'agisse de coupes en bas âge ou de récoltes dont les produits sont déjà utilisables, la question estici bien limitée: c'est la valeur que les coupes ont pour le propriétaire, c'est le prix où elles entrent dans la constitution de l'exploitation qu'il faut établir et non la somme qu'on en retirerait, en réalisant les produits par un abatage prématuré.

Après avoir analysé la forêt, c'est-à-dire, après avoir déterminé la valeur du sol, du matériel initial et du revenu à l'âge d'exploitation adopté par le propriétaire, on en déduira le taux de placement,

$$
\log \cdot(1+x)=\frac{\log \cdot \mathrm{R}+\mathrm{C}-\log \cdot \mathrm{C}}{n}
$$


On calculera ensuite les récoltes acquises comme étant égales aux intérêts composés à ce taux du fonds générateur C :

$$
C\left[(1+t)^{n}-1\right]
$$

Exemple : un taillis ordinaire rapportant 980 francs à 30 ans sur un fonds de 542 franes (sol 420 , ensouchement 122) forme un placemsent à $31 / 20 / 0$; les récoltes acquises sont fournies en multipliant 542 par les facteurs du tarif $I$, dont on a retranché l'unité.
A 1 an....... $542 \times 0,035$ récolte $188^{\text {r }} 97$
A 2 ans....... $542 \times 0,071-3848$
A 3 ans....... $542 \times 0,108-5853$
A 4 ans....... $542 \times 0,147 \quad-7967$
A 5 ans....... $542 \times 0,187-10135$
A 6 ans....... $542 \times 0,229-12412$
A 7 ans....... $542 \times 0,272-14742$
A 8 ans....... $542 \times 0,316-17127$
A 9 ans....... $542 \times 0,363-19674$
A 10 ans....... $542 \times 0,410-22222$
A 15 ans....... $542 \times 0,675-36575$
A 20 ans.. .... $542 \times 0,989-53604$
A 25 ans....... $542 \times 1,363-73875$
A 30 ans....... $542 \times 1,807-980$ "

Les valeurs qu'on obtient ainsi pour lés récoltes pendantes sont essentiellement relatives et ne concernent que le propriétaire; elles ne se confondent arec; la superficie que dans le cas où la forêt est exploitée par coupes de blanc estoc comme les taillis ordinaires. Le propriétaire victime d'un dommage est toujours libre de s'entendre avec l'auteur de ce dommage pour assigner aux bois la valeur vénale absolue résultant de leur utilisation possible; nous pensons qu'en cas de contestation, il n'y aurait pas d'autre règle d'évaluation que celle qui vient d'être indiquée.

$\mathrm{N}^{\circ}$ 147. - Bien souvent des inspecteurs de compagnies d'assurances ont demandé à l'École forestière une table donnant la valeur des taillis à leurs différents âges, comme si la valeur ne dépendait que de l'âge el ne variait pas 
avec les essences les moyens de vidange et les conditions économiques de chaque localité. Leur elreur était d'ailleurs fort excusable, car certains auteurs n'ont pas hésité à dresser des tables pour assigner des prix fixes aux coupes de chaque âge et n'ont pas craint de les déclarer applicables à toutes les forêts de France ( $\left.{ }^{1}\right)$.

On doit, pour chaque forêt et dans chaque cas particulier, calculer soi-même au moyen de la règle précédente les valeurs relatives des récoltes acquises et il n'y a de relativement vrai que la table dressée pour l'exploitation dont on a déterminé les conditions.

Le calcul étant plus compliqué pour les forêts à arbres de réserve qui s'exploitent par coupes à tire-aire comme les taillis sous futaie, nous en donnons l'exemple suivant:

Un hectare de taillis sous futaie produit $1,110 \mathrm{fr}$. à 30 ans (futaie $610 \mathrm{fr}$., taillis $500 \mathrm{fr}$.). Le capital engagé et de $780 \mathrm{fr}$., savoir: $420 \mathrm{fr}$. pour le sol, $122 \mathrm{fr}$. pour l'ensouchement et $238 \mathrm{fr}$. pour le balivage initial. Dans ces conditions, le taux du placement ést de $30 / 0$ : le calcul indiqué au numéro précédent fournirait les valeur's successives des lécoltes en croissances, mais il est utile de distinguer dans ces récoltes la partie en taillis et la partie due au grossissement des arbres; il suffira de diviser le capital générateur 780 en deux parties génératrices des revenus 500 et 610 :

$$
\begin{aligned}
& 780 \times 1,427=500+610 \\
& \frac{500}{1,427}=353^{r} \text { pour le taillis } \\
& \frac{610}{1,427}=\frac{427 \text { pour la futaie }}{780^{\mathrm{r}}}
\end{aligned}
$$

Les récoltes ainsi calculées à $30 / 0$ pour le taillis et la futaie fourniront la superficie en y ajoutant la valeur du balivage initial $238 \mathrm{fr}$.

(1) M. Chabanne, Tarifs on comples faits de la valeur progressive d'un hectare de taillis à difiérents prix avec intérêts conposés au taux de $ّ 0 / 0$. - M. Noirot-Bonnet, Manuel de l'estimateut des foréts et Maison rustique du XII'e siècle, tome $\mathrm{IV}$. 


\begin{tabular}{|c|c|c|c|c|c|}
\hline \multirow[b]{2}{*}{ Ages. } & \multicolumn{3}{|c|}{ RÉCOLTE } & \multicolumn{2}{|c|}{ VALEUR } \\
\hline & en taillis. & en futaie. & totale. & de la futaie. & de la superficie \\
\hline 1 & 10,59 & 12,81 & 23,40 & 250,81 & 261,39 \\
\hline 2 & 24,71 & 29,89 & 54,60 & 267,89 & 292,60 \\
\hline 3 & 32,72 & 39,58 & 72,30 & 277,58 & 310,30 \\
\hline 4 & 44,10 & 53,07 & 97,17 & 291,07 & 335,17 \\
\hline 5 & 56,12 & 67,89 & 124,01 & 305,89 & 362,01 \\
\hline 6 & 68,48 & 82,84 & 151,32 & 320,84 & 389,32 \\
\hline 7 & 81,19 & 98,21 & 179,40 & 336,21 & 417,40 \\
\hline 8 & 94,25 & 114,01 & 208,26 & 352,01 & 446,26 \\
\hline 9 & 107,60 & 130,23 & 237,83 & 368,23 & 475,83 \\
\hline 10 & 121,43 & 146,88 & 268,31 & 384,88 & 506,31 \\
\hline 15 & 196,97 & 238,26 & 435,23 & 476,26 & 673,23 \\
\hline 20 & 284,52 & 344,16 & 628,68 & 582,16 & 866,68 \\
\hline 25 & 386,18 & $46 \tau, 14$ & 812,32 & 705,14 & 1051,32 \\
\hline 30 & $500 \gg$ & 610 & 1110 & $848 "$ & 1348 \\
\hline
\end{tabular}

On ne saurait trop observer qu'un pareil calcul n'est vrai que pour le cas où il s'agit de déterminer les prix auxquels les coupes entrent dans l'exploitation qu'on doit reconstituer en argent; il ne faudrait jamais étendre ces valeurs relatives aux autres questions de l'économie forestière ni les considérer comme les valeurs absolues des bois en croissance, car la végétation ne saurait. créer la valeur suivant cette loi mathématique.

$\mathrm{N}^{\circ}$ 148. - Il ne faut pas confondre la valeur pour laquelle une récolte entre dans le bilan d'une exploitation forestière avec le prix qu'en donnerait l'acheteur d'une coupe sur pied. A cet acheteur la constitution de l'exploitation reste étrangère; il achète une sorte de billet à échéance fixe, le vendeur restant propriétaire sans rémunération du sol et de son outillage. Rien donc de plus naturel que d'estimer la récolte à un pareil acheteur par la formule de l'escompte qui en ramène la valeur à l'actualité

$$
R=\frac{1}{(1+t)^{n}}
$$


L'acheteur d'une récolte sur pied en donnera nécessairement la somme qui, placée à intérêts pendant $n$ ans, lui procurera la valeur de cette récolte et le vendeur plaçant cette somme à intérêts composés retrouvera à son tour le prix que la coupe lui aurait fourni.

A quel taux doit s'opèrer cette réduction à l'actualité ? La loi de l'offre et de la demande peut seule régler cette question: or, comme on achète rarement des récoltes sur pied, il est fort difficile d'indiquer un taux quelconque.

Voici le calcul fait à $50 / 0$ et à l'aide du tavif II exprimant les prix de vente à différents âges d'une coupe de taillis ordinaire valant $980 \mathrm{fr}$. ̀̀ 30 ans.

Coupe de 29 ans escomptée pour 1 an.... 932 r 95

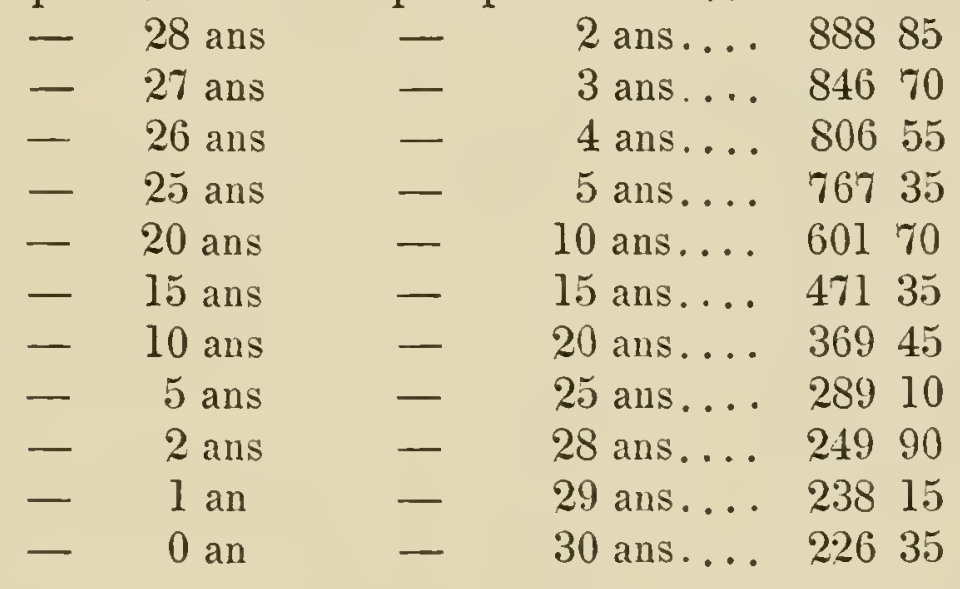

Il est évident que de pareils prix ne représentent ni la valeur absolue due aux marchandises qu'on pourrait en retirer, ni celle que peut créer la végétation dans sa marche ascendante, ni enfin, celle qui entre dans la constitution de l'exploitation erga dominum; mais il est non moins évident que celui qui voudrait acheter le droit à une récolte de $980 \mathrm{fr}$. dans 30 ans, avec la condition d'avoir son déboursé placé à $50 / 0$, pourrait donner $226 \mathrm{fr} .35$ de la récolte naissante, car cette somme capitalisée par le tarif $I$ fournirait au bout de 30 ans $980 \mathrm{fr}$.

$$
226,35 \times 4,322=980
$$

No 149. - La vente de récoltes sur pied se présente le 
plus souvent dans les expropriations pour cause d'utilité publique. Si la forêt est louée, il faut payer à deux ayantdroits la cession forcée qui leur est imposée. Naturellement on paiera au propriétaire le fonds générateur et au locataire la récolte en espérance. Naturellement aussi, si le propriétaire est en même temps son propre locataire, il doit céder, à la fois, le fonds générateur et la récolte, et aura droit à la double indemnité résultant de la double cession.

$$
\mathrm{C}+\frac{\mathrm{R}}{(1+t)^{n-m}}
$$

C étant le fonds générateur, sol, ensouchement, semis et balivage.

$R$ la valeur de Ja coupe mûre.

$n$ l'âge adopté pour l'exploitation.

$m$ l'âge du moment où se fait l'expropriation.

Le taux à employer ici ne peut être que celui du placement même, c'est-à-dire celui où les fonds engagés fonctionnent dans l'entreprise forestière.

La raison en est $: 1^{\circ}$ que pour l'exploitant le billet à échéance représenté par la coupe est bien une valeur placée à ce taux; $2^{\circ}$ qu’un fermier louant la forêt à $980 \mathrm{fr}$. serait bien acheteur à ce même taux du billet représenté par la coupe $3^{\circ}$ dans l'impossibilité de trouver la mesure des transactions dans les ventes de récoltes faites dans la localité, le taux de placement des fonds engagés dans la forêt est le plus naturel et le moins sujet à discussion.

Voici pour le taillis précédent, rapportant $980 \mathrm{fr}$. à 30 ans avec un sol ensouché de $542 \mathrm{fr}$., formant placement à $31 / 20 / 0$, les valeurs de cession forcée des récoltes à différents âges :

Coupe de 29 ans escomptée pour 1 an.... $946^{\text {f }} 70$

$$
\begin{aligned}
& \text { - } 28 \text { ans - } 2 \text { ans... } 91435 \\
& \text { - } 27 \text { ans }-3 \text { ans... } 88395 \\
& \text { - } 26 \text { ans - } 4 \text { ans... } 85360 \\
& \text { - } 25 \text { ans }-5 \text { ans... } 82515
\end{aligned}
$$


Coupe de 20 ans escomptée pour 10 ans... $694^{\text {r }} 80$

$\begin{array}{cccccc}\text { - } & 15 \text { ans } & - & 15 \text { ans... } & 58505 \\ \text { - } & 10 \text { ans } & - & 20 \text { ans... } & 49295 \\ \text { - } & 5 \text { ans } & - & 25 \text { ans... } & 41450 \\ \text { - } & 2 \text { ans } & - & 28 \text { ans... } & 37435 \\ \text { - } & 1 \text { an } & - & 29 \text { ans... } & 36065 \\ \text { - } & 0 \text { an } & - & 30 \text { ans... } & 34890\end{array}$

L'expropriè qui cède la coupe naissante pour 348 fr. 90 , reçoit ainsi une somme qui, placée á $31 / 20 / 0$ pendant 30 ans, reproduit $980 \mathrm{fr}$.

$$
348,90 \times 2,80 \tau=980
$$

Il ne faut pas confondre ce cas avec celui de la réparation du dommage causé par un incendie analysé $n^{\circ} 146$. Pour évaluer ce dommage, il s'agit de reconstituer l'exploitation telle qu'elle était auparavant; rien n'est plus naturel que de chercher le prix auquel la récolte entre dans la composition de l'exploitation. Ici, au contraire, il y a vente forcée du fonds et vente, non moins forcée, de la récolte en espérance. Comme dans le champ de blé qu'on exproprie au moment où la récolte est en vert, il faut donc payer avec la valeur du fonds générateur (sol garni de son ensouchement et du balivage), l'espérance d'une récolte qui ne se produira plus ( ${ }^{1}$ ).

Ainsi, dans l'exemple ci-dessus, où le capital générateur est estimé $542 \mathrm{fr}$. (sol, $420 \mathrm{fr}$.; ensouchement, $12 \% \mathrm{fr}$.), l'indemnité d'expropriation pour un hectare âgé de 25 ans sera :

$$
542+825,15=1367,15
$$

et pour un hectare âgé de 1 an, elle sera de :

$$
542+360,65=902,65
$$

Vainement, dira-t-on : l'expropriant paie relativement plus cher pour un bois îgé de 1 an que pour celui âgé de 25 ans; la raison en est fort simple : le bois de 1 an est tout en espérance, celui de 25 ans est beaucoup plus près de la récolte. Par le paiement de cette indemnité, l'expro-

(1) Estimations concernant la proprieté forcstière, no ¿̃2. 
priant devient propriétaire du fonds et de la récolte. S'il abandonne les bois à l'exproprié, c'est en déduction de l'indemnité qu'il devra payer.

Or, il est certain que, dans un bois de 25 ans, l'exproprié retirera des marchandises pour un prix à peu près égal et que l'expropriant n'aura, dès lors, que peu de chose à payer en outre du fonds générateur. Pour le bois de 1 an, au contraire, l'exproprié auquel on abandonne la superficie ne retirera rien ou presque rien de la récolte anticipée, il est juste et naturel que l'expropriant l'en indemnise en lui payant une somme relativement plus considérable. Il en est de unéme pour la récolte de blé qu’on exproprie au moment où il est en vert; la somme due au fermier diminue à mesure que le blé approche de sa maturité, quand on lui abandonne la récolte en déduction de l'indemnité qu'il doit recevoir.

Article 3.

\section{Comparaison agricole.}

Nos 150. - Fente du sol. - Sa définition, calcul de certains auteurs.

131. - Fausse application à la recherche de l'àge d'exploitation la plus avantageuse au point de vue du tanx de placement.

1ว2. - Fausse application à la comparaison des exploitations agricoles.

133. - Comparaison agricole. - Le taux de placement est le mojen le plus simple de comparer les exploitations.

154. - Conditions dn problème.

15๊. - Arantages des placements en forêts à capital égal.

156. - Exploitations agricoles de moindre capital.

157. - Conclusion .

No 150. - Rente du sol. - L'examen des valeurs assignées aux récoltes en croissance, quand on les calcule au taux de placement de l'exploitation, c'est-à-dire erga 
dominum, a conduit certains auteurs à des conceptions purement théoriques dont il importe de dire quelques mots.

La première récolte, celle qui est acquise à un an, s'exprime par :

$$
\mathrm{C} t \text { ou } \mathrm{R} \frac{t}{(1+t)^{n}-1}
$$

$\mathrm{C}=$ prix assigné au capital générateur, c'est-à-dire au sol garni de son matériel d'exploitation (souches, semis ou balivage) ;

$\mathrm{R}=$ valeur du revenu à $n$ ans;

$n=$ terme assigné à l'exploitation;

$t=$ taux de placement, résultant de la relation d'intérêts composés existant entre $\mathrm{C}$ et $\mathrm{R}$.

Exemple: Si on se reporte au taillis ordinaire du $\mathrm{n}^{\circ} 122$, au taillis sous futaie composée du $n^{\circ} 122$, et à la sapinière du $\mathrm{n}^{\circ} 144$, on trouve pour valeur de la première récolte :

$$
\begin{aligned}
& \text { Taillis ordinaire...... } 542 \times 3,50 / 0=18,97 \\
& \text { Taillis sous futaie..... } 580 \times 30 / 0=23,40 \\
& \text { Sapinière........ } 500 \times 2,50 / 0=12,50
\end{aligned}
$$

$1^{\circ}$ On a appelé feuitle, la valeur produite chaque année par la végétation.

La feuille de la première année est ainsi l'intérêt du fonds grénérateur au taux de placement de l'exploitation.

C'est également l'annuité (économie) qui, placée tous les ans au taux $t$ produit $R$ en $n$ ans, ou, en d'autres termes, l'annuité génératrice de $\mathrm{R}$ au taux $t$.

La feuille de seconde année est cette annuité grossie de l'annuité précédente et de ses intérêts pendant un an. La troisième feuille est représentée par l'annuité constante à laquelle s’ajoutent les intérêts composés des annuités antérieures, etc.

On conviendra qu'une pareille conception est un simple jeu de calcul, car la végétation ne saurait créer les valeurs par cette loi des annuités, identique, du reste, à celle des 
intérêts composés ( $n^{\circ} 37$ ). Qu'on donne le nom de feuille aux différences r'ésultant de l'accumulation de cette annuité ou des intérêts composés, cela n'a pas grande importance; l'essentisl est de se rappeler toujours que les valeurs ainsi calculées sont retatives au propriétaire et expriment seulement le prix auquel les récoltes entrent dans la constitution de son exploitation ( $\left.n^{\circ} 146\right)$.

$2^{\circ}$ D'autres forestiers, économistes plus physiocrates que modernes, ont dit que cette première récolte était la rente du sol; cette première feuille ainsi calculée a été également appelèe rente foncière.

On sait que la rente d'un sol n'est autre chose que le prouluit de l'exploitation dégagé de tous les frais inhérents à l'entreprise; c'est la rente de l'exploitation $\left(n^{\circ} 114\right)$ dont on a défalqué les intérêts du capital d'exploitation.

La rente forestière ou rente de l'exploitation étant :

$$
\frac{\mathrm{R}}{n}
$$

La rente foncière ou rente du sol serait:

$$
\frac{\mathrm{R} t}{(1+t)^{n}-1} \text {. }
$$

Dans les forêts qui nous servent d'exemple, on aurait:

Taillis ordinaire. Rente de la forêt 32,66 Rente du sol 18,97 $\begin{array}{llllll}\text { Taillis sous futaie } & - & 37 & - & 23,40\end{array}$ Sapinière..... - $\quad 110 \quad-\quad 12,50$

Ces forestiers ont considéré la première feuille $\mathrm{C} t$ comme étant la véritable rente foncière, c'est-à-dire le produit même du terain et ils ont justifié leur dire, en montrant que si on retranche de la rente de la forêt'intérêt du capital-bois immobilisé pour constituer l'exploitation aménagée, on obtient précisément la valeur de la première feuille.

En effet, la rente de la forêt étant $\frac{R}{n}$, il faut en retran- 
cher l'intérêt du matériel-bois $M$ de l'exploitation aménagée; or, $\left(\mathrm{n}^{\circ} 144\right)$

$$
\mathrm{M}=\frac{\mathrm{R}}{t}-n \mathrm{C}=\frac{\mathrm{R}}{t}-\frac{n \mathrm{R}}{(1+t)^{n}-1}
$$

Le matériel par hectare est done :

L'intérèt de ce matériel est :

$$
\frac{\mathrm{R}}{\mathrm{tn}}-\frac{\mathrm{R}}{(1+t)^{n-1}}
$$

$$
\frac{\mathrm{R}}{n}-\frac{\mathrm{R} t}{(1+t)^{n}-1}
$$

Effectuant la soustraction qui donne la rente du sol, on obtient :

$$
\frac{\mathrm{R}}{n}-\frac{\mathrm{R}}{n}+\frac{\mathrm{R} t}{(1+t)^{n}-1}=\mathrm{R} \frac{t}{(1+t)^{n}-1}=\mathrm{C} t
$$

Ainsi dans l'exemple de la sapinière de 140 hectares aménagée à 140 ans, produisant $15,400 \mathrm{fr}$. par an et valant $616,000 \mathrm{fr}$., savoir :

Matériel-bois........... $546000^{\mathrm{f}}$ "

Sol et semis............. 70000 "

Le taux de placement ressol't à $21 / 2$ :

$$
\frac{15400}{616000}=2,5
$$

La rente du sol est.

$$
500 \times 2,5=12,50
$$

car le matériel par hectare étant de :

$$
\frac{546000}{140}=3900
$$

L'intérêt de 3,900 à $21 / 20 / 0$ étant de 97,50 , la rente du sol est bien :

$$
110-97,50=12,50
$$

Dans les conceptions de cette nature, il y a toujours un mélange de vérité et d'erreur : la vérité est que la première feuille ainsi calculée est bien le produit du fonds générateur dégagé des intérêts des récoltes pendantes qui entrent dans le matériel de l'exploitation aménagée. L'erreur consiste à croire que cette première feuille représente la rente du sol.

$1^{\circ}$ On oublie, en effet, que dans les exploitations à coupes de blanc estoc, le fonds C n'est pas le sol seul, mais un élément générateur composé du sol et d'un accessoire 
important formé de l'ensouchement ou du semis initial et que, dans les exploitations à arbres de réserve, le balivage fait, en outre, partie de ce fonds et il faudrait en défalquer l'intérêt;

$2^{\circ}$ On suppose que les récoltes pendantes ont des valeurs croissant suivant la loi de l'intérêt composé, ce qui est une hypothèse fausse ( $n^{\circ}$ 142); de sorte que l'on défalque de la rente de l'exploitation, l'intérêt d'une quantité trop faible $\left(n^{\circ} 143\right)$ ce qui vicie l'expression de la rente du sol d'une manière indéterminée ;

$3^{\circ}$ On omet, enfin, que l'exploitation peut être dotée d'un autre capital que le matériel-bois et qui consiste dans la valeur des routes, maisons, scieries, etc., etc.

En réalité, on n'a déduit de la rente de l'exploitation que l'intérêt des récoltes pendantes et non l'intérêt de tout le capital engagé. On conçoit que si cet ingénieux calcul peut, à la rigueur, être employé pour les forêts exploitées à blanc estoc, il ne saurait dans tous les cas représenter la véritable rente du sol.

$N^{\circ} 151 .-$ Cependant les inventeurs de cette rente foncière ont cru y trouver la solution d'un problème économique important: à quel âge faut-il régler la coupe d'une exploitation forestière pour obteni. la rente du sol la plus élevée ? Problème qui dans leur pensée assure l'exploitation la plus avantageuse au point de vue pécuniaire et qui a recu le nom d'exploitabilité commerciale.

Les capitaux, ont-ils dit, s'emploient dans une localité en fonds ruraux à un taux moyen, habituel et connu, $30 / 0$ par exemple : évaluons d'après les forêts voisines le revenu $\mathrm{R}$ probable de l'exploitation à diffẻrents âges, par exemple :
A 20 ans .......... R $=400^{\text {f }}$ w
A 25 ans ............ 600 ,
A 30 ans............. 925 ,
A 35 ans............ 1200 "
A 40 ans............ 1450 D 
Calculons le fonds générateur $\mathrm{C}$ par la formule du tarif III, et la rente du sol à $30 / 0$ :
A 20 ans...........
A 25 ans.............
$\mathrm{C}=496^{\mathrm{f}}$
$\mathrm{R}=14,88$
A 30 ans.............
548 》 16,44
A 35 ans............
648 》
19,44
A 40 ans...........
$661 \gg$
19,83
$641 \gg$
19,23

La rente du sol étant le plus élevẻe à 35 ans, c'est, dit-on, à cet âge qu'il faut régler la coupe pour obtenir la rente la plus élevée dn terrain traité en forèt.

Pour abréger le calcul et pour simplifier la solution du problème, on a conseillé de calculer les capitaux générateurs $\mathrm{C}$ en multipliant les revenus $\mathrm{R}$ par les facteurs du tarif III correspondant au taux admis $l$; et on a dit que la rente la plus élevée du sol arrivait à l'âge oủ le produit était maximum (661 fr. à 35 ans, dans notre exemple).

Or, nous le demandons à tout homme impartial, est-il permis de soutenir avec la moindre apparence de raison, que la coupe faite ainsi à 35 ans assurera la rente du sol la plus élevée.

Vous n’avez pas estimé le prix de votre terrain, vous ne connaissez pas la valeur de votre capital engagé, et vous avez la prétention de soutenir que vous avez déterminé non seulement la rente de ce sol inconnu, mais encore son maximum!

Vous avez défini la rente foncière « ce qui reste du produit de l'hectare, déduction faite de l'intérêt du capital engagé " et, non seulement vous confondez le sol et son matériel initial, vous négligez les autres capitaux immobilisés dans la forêt, mais en outre, vous supposez que l'intérêt du capital-bois des récoltes pendantes, le seul que vous comptez, est égal au taux moyen des exploitations rurales.

Vous supposez, de plus, que les valeurs des récoltes qui entrent dans la composition du matériel-bois de l'exploitation suivent une marche ascendante égale à celle de la formation des intérêts à ce taux des exploitations 
rurales, comme si la végétation créait les valeurs forestières en suivant cette loi mathématique de l'intérèt composé à un taux arbitrairement déterminé, taux moyen, intermédiaire entre celui des placements en labours, en prairies, en vignes et en forêts.

N'est-ce pas là une conception de pure fantaisie et une de ces nébulosités qu'on a tant reprochées aux forestiers?

Nous n'insisterons pas davantage pour démontrer l'erreur d'une solution que nous avons critiquée ailleurs (').

Nous avons voulu seulement domner ici une autre forme à nos critiques pour les rendre plus tangibles.

Le seul problème pratique est celui qui consiste à trouver l'âge qui correspond au maximum du taux de placement du capital engagé : à cet âge, on aura l'exploitation qui fournit l'emploi des capitaux au taux le plus avantageux possible dans la limite où nos connaissances sur la marche de la végétation permettent d'arriver à la solution de la question ( $\mathrm{n}^{\circ} 131$ ). Nous en avons donné la solution ( $n^{\circ}$ 134), celle-ci ne peut s'obtenir que par des essais successifs de la formule générale du taux de placement ( $\left.n^{0} 124\right)$ ou, si on veut plus de rapidité, par des essais de la formule simplifiée :

$$
(1+x)^{n}=\frac{\mathrm{R}+\mathrm{C}}{\mathrm{C}}
$$

dans laquelle $\mathrm{C}$ et $\mathrm{R}$ se déterminent directement: $\mathrm{R}$ par l'exemple des forêts voisines de même qualité à divers âges $n$, et $\mathrm{C}$ par l'estimation directe du sol, du matérielbois et du capital ordinaire. $C$ et $R$ varient en général à chaque âge; mais dans les exploitations à coupes de blanc estoc, $\mathrm{C}$ peut être considéré comme constant pour une même forêt, tandis que dans les exploitations à arbres de réserves, $\mathrm{C}$ varie, à cause de son balivage, avec l'âge d'exploitation, car la valeur marcliande d'un méme bali-

(1) Estimations concernant la propriété forestière, note p. 249. 
vage est plus grande à 30 qu'à 25 et 20 ans. N'est-on pas en droit de critiquer ceux qui ont crutrouver une formule donnant le maximum de $t$ dans une expression dont tous les éléments $\mathrm{C}$, $\mathrm{R}$ et $n$ sont des variables?

$\mathrm{N}^{\circ}$ 152. - Cette fausse conception de la rente du sol a été employée pour comparer les résultats de l'exploitation forestière à ceux que fournit l'agriculture et pour éclairer l'économiste sur la supériorité ou l'infériorité de la forêt vis-à-vis du chainp ou du pâturage.

Une sapinière de 140 hectares aménagée à 140 ans sur un sol qui vaut avec le semis $500 \mathrm{fr}$. l'hectare, produit un revenu annuel de $15,400 \mathrm{fr}$.

$$
\begin{aligned}
\log .(1+x) & =\frac{\log .15900-\log .500}{140} \\
x & =21 / 20 / 0
\end{aligned}
$$

Rente du sol $=500 \times 2,50 / 0=12,50$

Si cette forèt peut ètre défrichée et transformée en ferme lonée 30 à $3 \breve{f r}$. par hectare, on en conclut que la culture agricole produit des résultats bien supérieurs à ceux de la forêt.

Nous reconnaissons que la rente du sol est un moyen d'exprimer la comparaison; mais c'est à la condition que cette rente soit exactement calculée et que les illusions ne soient point prises pour des réalités.

En ce qui concerne la forêt, nous avons vu que le calcul précédent est erroné : on évalue faussement la rente du sol, car on défalque seulement de la rente de l'exploitation l'intérêt des récoltes pendantes qui sont une partie et non la totalité du matériel d'exploitation, enfin on omet l'intérêt du capital d'amélioration (chemins, maisons de garde, scieries, etc.).

Pour l'exploitation agricole, la rente nette du sol est plus délicate encore à calculer; le capital qui y est consacré est de double nature: une partie est fixe (bîtiments, chemins, canaux, drainage, etc.), l'autre est mobile (bétail, instruments, semences, engrais, etc.). A la rému- 
nération, c'est-à-dire à l'intérêt de tout ce capital, il faut ajouter son amortissement, son entretien, son assurance, les salaires des ouvriers et le légitime traitement de l'exploitant. Il faut tenir compte, en outre, des différents modes de tenure, fermage, métayage et faire-valoir direct, dans lesquels le capital d'exploitation est, soit divisé entre le propriétaire et le fermier, soit mis en société avec le métayer, soit, enfin, fourni en totalité par le propriétairecultivateur.

Or, dans le calcul précédent, on a confondu le fermage avec la rente du sol; on a omis de défalquer l'intérêt des bâtiments, leur entretien, leur usure, leur assurance et les pertes résultant des mauvais fermiers.

Le calcul suivant, dans lequel nous comparons la forêt à une ferme louée à la suite du défrichement, montre la manière dont les choses doivent être raisonnées.

La forêt. Les fonds engagés dans l'exploitation d'une sapinière de 140 hectares, aménagée à 140 ans, sont représentés par :

Terrain.........6 60900

\begin{tabular}{|c|c|c|c|c|}
\hline Semis. & 9100 & ” & - & 65 \\
\hline Matériel-bois. & 546000 & 1 & - & 3900 \\
\hline Routes et clôtures. & 25200 & » & 一 & 180 \\
\hline Tota & 64 & I) & & \\
\hline
\end{tabular}

Le revenu annuel net est 15,400 fr., ainsi détaillé :

Coupe principale, éclaircies, etc. $16800^{\mathrm{r}}$ " soit $\mathrm{p}^{\mathrm{r}}$ hect. $120^{\mathrm{r}}$ " Garderie........ 500

Frais de gestion.... 150 " 1400 " -10 "

Entretien des chemins 750 "

Le toux du placement est

$$
\frac{15400}{641200}=2,410 / 0
$$

La rente de l'exploitation est de $110 \mathrm{fr}$.

La rente du sol est de $10 \mathrm{fr} .10$ :

$$
\begin{gathered}
2,410 / 0 \text { de } 4145=99,90 \\
120-10-99,90=10,10
\end{gathered}
$$


La ferme. Capital engagé :

\begin{tabular}{|c|c|c|c|c|}
\hline Sol & $60900^{f}$ & & soit par hectare & $435^{\mathrm{f}}$ \\
\hline Semis sacrifiés.... & 9100 & ) & - & 65 \\
\hline Défrichement.... & 1400 & 》) & - & 100 \\
\hline Bâtiments........ & 40600 & " & - & 290 \\
\hline Chemins et clòtures. & 25200 & " & - & 180 \\
\hline Total & $149800^{\mathrm{f}}$ & » & 一 & $635^{\mathrm{f}}$ \\
\hline
\end{tabular}

Le revenu net est 4,200 fr., savoir :

Fermage ...... $4900^{\mathrm{f}}$ 》 soit par hectare $35^{\mathrm{f}}$ ” Réparations $600^{\mathrm{r}}$ ") 700 ” $\quad$ - 5 Assurance. $100^{\mathrm{r}} \mathrm{m}$

$$
\left.4200^{\mathrm{f}}\right)
$$

$30^{\mathrm{f}}$

Le taux du placement est

$$
\frac{4200}{149800}=2,800 / 0
$$

Rente nette de l'exploitation : $30 \mathrm{fr}$.

Rente du sol : $12 \mathrm{fr}$. 22.

$$
\begin{gathered}
2,800 / 0 \text { de } 635=17,78 \\
35-5-17,78=12,22
\end{gathered}
$$

On voit par cet exemple que la transformation si favorable en apparence à la ferme ne donne, en réalité, qu'un placement de $2,800 / 0$ au lieu de 2,41 et une rente foncière de 12,22 au lieu de 10,10. Les chiffres de l'exemple précédent peuvent varier suivant les situations, ils n'ont ici pour but que d'indiquer les bases du calcul et non de conclure d'une manière systématique en faveur de la forêt.

$\mathrm{N}^{\circ}$ 153. - Comparaison agricole. - Tout en reconnaissant qu'un exact calcul de la rente du sol peut utilement servir à établir la comparaison, nous pensons que cette considération est plus spécieuse qu'utile et qu'on doit la remplacer par le calcul du taux de placement des fonds engag'és dans l'exploitation, qui suffit à mesurer les choses d'une manière simple et pratique. Quand on dit, par exemple, que l'exploitation forestière assure 2,40 0/0 des fonds qui y sont engagés, et que la ferme rapporte 2,80 0/0, la comparaison est aussi nette et aussi complète 
que celle qui consiste à dire que les fonds confiés à la forêt font rendre à la teme $10 \mathrm{fr} .10$ par hectare et que ceux mis dans la ferme lui font produire $12 \mathrm{fr} .22$.

La considération de la rente du sol est inutile puisque il faut pour l'exprimer calculer d'abord le taux de placement et que celui-ci suffit à tout mesurer.

Elle est spécieuse et surannée, car, au temps où les premiers économistes considéraient la terre comme l'élément producteur de toute richesse, on pouvait concevoir qu'il était logique de rapporter tous les produits au sol, en retranchant les frais et notamment les intérêts du capital qui lui était confié. Aujourd'hui, la science économique, devenue plus simple et plus positive, ne considère que le résultat final. La terre dont la propriété a été acquise par l'échange d'une somme d'argent est devenue un capital comme les bâtiments de la ferme et les lécoltes pendantes de la forêt. Nulle production ne s'opère sans capital : à quel titre, dès lors, rapporter les produits à une partie des capitaux engagés, plutôt qu'à l'autre? Pourquoi calculer la rente de la terre, plutôt que celle du capital fixe ou du capital mobile?

On voit donc que la rente du sol est une conception surannée et tout à fait inutile, le calcul du taux de placement suffit à toutes les comparaisons, car il est la mesure simple du revenu par rapport aux capitaux de toute nature qui sont engagés dans l'entreprise.

$\mathrm{N}^{\circ}$ 154. - L'instrument de mesure étant admis, il faut que le problème soit exactement posé, afin qu'on ne s'égare pas en des considérations étrangères à la comparaison entre l'agriculture et la sylviculture.

" Pour un sol de même qualité et valeur, un même " capital est-il susceptible d'une rémunération plus avan" tageuse en culture agricole qu'en forêt? En d'autres " termes, le taux de placement d'une même quantité de " fonds engagés, avec la condition que le sol soit de 
" même valeur de part et d'autre, est-il plus élevé ou " plus faible dans l'industrie agricole que dans l'exploi" tation forestière? ")

Telle est la question qui seule peut être posée: la condition dont elle dépend est indispensable, car on ne saurait jamais comparer que ce qui est de même nature. Les forêts sont généralement implantées sur des sols que l'agriculteur a dédaignés de longue date; les terres à bois sont souvent de médiocre qualité ; presque toujours homogènes, elles ne présentent pas la variété nécessaire aux cultures diverses d'une ferme bien constituée; l'eau y fait souvent défaut, ce qui empêche la création de prairies naturelles. De là sont venus les plus grands mécomptes qui ont suivi les défrichements inconsidérés; de là naît la raison pour laquelle tant de terrains défrichés imprudemment retournent actuellement à la forêt par des reboisements coûteux.

Il faut ajouter qu'on devra comparer seulement les modes d'exploitation analogues, c'est-à-dire, la forêt dont on vend les coupes sur pied à la ferme louée, ou l'exploitation du propriétaire forestier débitant ses bois à celle du propriétaire agricole cultivant lui-même sa terre. Dans le premier cas, les propriétaires restent libres de leur temps, qu'ils peuvent consacrer à un autre travail ; dans le second, l'exploitation directe absorbe tous leurs soins et leur intelligence. Le propriétaire qui loue son domaine fait avec le fermier un partage des produits dans lequel il est loin d'avoir part égale; celui qui le cultive en recueille seul tous les produits. Il en est de même des propriétaires de forêts et il y a des degrés infinis dans l'utilisation des bois : celui qui, dans l'exemple ( $\left.\mathrm{n}^{\circ} 152\right)$, consacrerait un supplément de capital à construire une usine destinée à débiter les sapins en planches, moulures, bois de menuiserie et de charpente, retirerait peut-être un revenu double de sa forêt, mais il serait obligé d'y consacrer tout son temps comme le cultivateur qui fait valoir directement sa ferme. 
Il ne faut donc mettre en comparaison que des situations identiques sinon du moins à peu près analogues.

No 155. - Les conditions du problème étant ainsi déterminées, on peut affirmer sans s'exposer au danger des généralisations trop absolues, qu'à égalité de capital confié à une même terre, l'exploitation forestière fournit un placement très souvent plus avantageux que la ferme louée et résultant du défrichement.

Prenons pour exemple 30 hectares de taillis sous futaie aménagés à 30 ans et valant $37,000 \mathrm{fr}$. ainsi détaillés :

Sol : 30 hectares à $420^{\mathrm{f}} \ldots \ldots \ldots \ldots \ldots \ldots \ldots$. $12600^{\mathrm{f}}$ n

Eusouchement : 30 hectares à 122 f......... 3660 » Superficie $\left\{\begin{array}{l}\text { balivage }: 30 \text { hect. à } 238^{\mathrm{f}} .7140^{\mathrm{f}} " \\ \text { récoltes pendantes...... } 13600 \text { " }\end{array}\right\} 20740$ "

Total ..........

Le revenu net de la forêt étant de... $1110^{\text {f }}$ ”

Le taux de placement est de...... $30 / 0$

La rente de l'exploitation est de... 37 》

Le propriétaire, étant certain de louer la ferme à $45 \mathrm{fr}$. l'hectare, se décide à défricher la forêt, à construire les bâtiments et à constituer un domaine agricole qui lui revient à 37,000 fr., savoir :

Valeur du terrain..................... 12600 "

Perte de l'ensouchement ................ 3660 »

Défrichement : 30 hectares à $100^{\mathrm{f}} \ldots \ldots \ldots \ldots \ldots 3000$ "

Construction des bâtiments, fontaines, etc...... 17740 ”

Total ............. $37000^{\text {f }}$

Mais le fermage étant de............. 1350

Il faudra en déduire chaque année:

Pour l'entretien des bâtiments....... 250

Leur assurance................. 100 " $\left.\begin{array}{l}\text { Provision pour pertes de fermage et } \\ \text { grosses réparations............. }\end{array}\right\}$

Revenu net......... $95 \overline{950^{\mathrm{f}}}$

Le taux de placement sera............ 2,56 0/0

$$
\frac{950}{37000}=2,560 / 0
$$

Et, encore, il faut supposer que les fermiers possédant 
le matériel de la culture ne viendront jamais à manquer à cette ferme, - que le fermage se maintiendra à $45 \mathrm{fr}$. l'hectare - et qu'il n'y aura de mécomptes ni dans la qualité de la terre, ni dans les conditions de la population agricole! Le propriétaire aura toujours à compter avec deux faits économiques trop fréquents pour ne pas être indiqués.

Le premier est que cette ferme qui lui coûte 37,000 fr. n'aura pas toujours cette valeur : les bâtiments, très onéreux à établir, perdent vite une notable partie de leur prix de construction. Les bâtiments s'incorporent si bien à la ferme que, sans eux, la terre n'a pas de valeur et qu'ils n'ont eux-mêmes aucune valeur sans la terre. Si, dans la localité, les domaines de cette nature s'achètent à 3 ou à 4 0/0 de leur revenu net, la ferme, qui revient à $37,000 \mathrm{fr}$., ne vaudra bientôt plus que $31,650 \mathrm{fr}$. ou même $23,750 \mathrm{fr}$.

Le capital affecté à bâtir une ferme s'amoindrit comme celui qui sert à meubler une habitation dès que la maison est construite ou que le mobilier est entré dans l'habita- • tion. On serait effrayé, si on supputait la quantité de travail et d'argent qui, depuis des siècles, a été confiée à la terre, sans que la rémunération en ait toujours été acquise et sans que la terre ait augmenté de valeur.

Le second fait économique est que depuis 50 ans les produits de l'agriculture n'ont pas sensiblement augmenté de prix, tandis que les bois ont progressé notablement; le propriétaire agricole est donc exposé à voir son domaine diminuer de revenu et de valeur: le mieux qui puisse lui arriver est de ne pas le voir s'anoindrir. Le propriétaire de la forêt a toujours la légitime espérance de voir la fortune lui sourire sous la double forme du revenu et du capital $\left({ }^{1}\right)$.

(1) Consulter à cet égard II. Guyot, professeur à l'école forestière : Histoire d'un domaine rural en Lorraine, Nancy 1887. 
Bien insensé serait donc celui qui, se laissant séduire par les illusions du fermage, se déciderait à défricher sa forêt pour placer sa valeur en une ferme bâtie. Mais, il faut le reconnaître, si la forêt appartient à un cultivateur possédant un peu d'argent pour se monter en bétail et en instruments agricoles, celui-ci aura intérêt à la détruire, à la défricher et à construire les bâtiments, parce que, sur cette exploitation agricole, il exercera son métier, y vivra et élèvera sa famille. Il y a, de ce côté, un danger pour la conservation des forêts: celles-ci pourront se transformer en petites exploitations agricoles d'égal capital. Ce danger dû an morcellement de la propriété forestière n'est pas un des moindres de ceux que nous avons déjà indiqués $\left(n^{\circ} 136\right)$ et dont il nous reste à compléter l'énumération.

$\mathrm{N}^{\circ}$ 156. - Il arrive souvent qu'une exploitation agricole exige beaucoup moins de capital que celui qui est affecté à une forêt de même étendue. Il en résulte des chances de destruction qui ne sont pas sans influence sur les ressources que les foréts sont appelées à fournir au travail national.

$1^{\circ}$ La sapinière indiquée au $n^{\circ} 152$ et estimée $641,200 \mathrm{fr}$. peut être défrichée et transformée en ferme coûtant $149,800 \mathrm{f}$., avec un taux de placement de $2,800 / 0$, au lieu de $2,410 / 0$. Le propriétaire réalisera de cette opération des bois pour 491,400 fr. qu'il emploiera en des placements mobiliers ou à des achats de domaines analogues.

$2^{\circ}$ Si la sapinière est située en montagnes et en région pastorale, ou le pâturage se loue facilement $20 \mathrm{fr}$. net par hectare, la transformation sera plus facile encore, car il suffira d'abattre les arbres, d'y introduire le bétail et de construire à peu de frais un mas ou chalet nécessaire à l'abri du bétail. Cette transformation coûtera, par exemple:

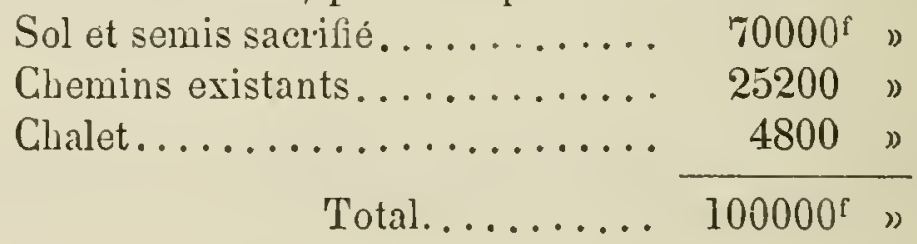


Le taux de placement sera de $2,800 / 0$ et le propriétaire pourra disposer de $541,200 \mathrm{fr}$.

Ces deux exemples, dont les chiffres peuvent varier suivant les circonstances, expliqueut les faits éconorniques suivants :

$1^{\circ}$ Les propriétaires de riches forêts sont souvent conduits par les besoins de la vie à réaliser le matériel de leurs forêts pour l'employer à des placements mobiliers plus rémunérateurs, tout en gardant le sol affecté à des exploitations rurales. C'est une tentation trop grande pour que certaines forêts, les plus belles, les plus riches en arbres n'y succombent pas au grand préjudice des besoins sociaux en bois d'œurre et de travail ;

$2^{\circ}$ Les forêts des montagnes pastorales sont les plus exposées à la destruction, parce que l'exploitation en pâturage s'établit presque sans frais et sans capital fixe. On voit par là combien la forêt a de peine à lutter contre le pâturagre dans les régions où le manque de chemins n'a pas encore créé la valeur au bois. On en déduit, aussi, que le meilleur moyen de défendre la forêt contre la dent du bétail est d'assurer la valeur à ses produits par le développement de la viabilité. Le plateau central, les Alpes et tant d'autres montagnes ont été déboisées par le pâturage, parce que le bois n'y avait point de valeur. L'Auvergne se reboise depuis 50 ans par l'influence de la route et du chemin de fer. Dans les Vosges, si bien percées et si bien outillées d'usines à débiter le bois, il n'entre plus une seule tête de bétail dans la forêt. La conservation des forêts par la valeur de leurs produits est d'ailleurs un fait général : les environs de Paris ont conservé leurs forêts de futaie, parce que la densité et l'industrie de la population ont assuré de tout temps la valeur de leurs produits.

No 157. - En réalité, les exploitations forestières et agricoles ne sont pas de même nature et ne sauraient être 
comparées. Aux différences caractéristiques qui ont été indiquées $\left(\mathrm{n}^{\circ} 86\right)$, il faut ajouter les deux remarques suivantes relatives au capital engagé.

$1^{\circ}$ Dans la forêt, le taux de placement diminue à mesure que l'îge d'exploitation s'accroît; mais le propriétaire augmente à la fois sa fortune et son revenu : il serait bien exigeant s'il ne se contentait pas d'un taux modeste qui le fait à la fois plus riche et mieux renté.

Dans la ferme, l'augmentation du capital n'accroît pas sensiblement le taux du placement; le capital améliore la quantité des produits qui restent, à peu près, dans une même relation avec les fonds engagés; ce capital doit toujours être reconstitué par des prélèvements sur le revenu.

$2^{\circ}$ Pour la forêt, la société est toujours exposée à des pertes d'épargnes par la nature mobilisable du matériel d'exploitation, celui-ci ne se reconstituant que par le temps et à longue échéance.

Pour l'agriculture, la société est protégée par la nature même du capital engagé : celui des bâtiments reste affecté à la terre, celui du bétail et des outils sert à monter d'autres exploitations qui se reconstituent en un temps fort court.

Il en résulte qu'au point de vue de l'action des pouvoirs publics, l'agriculture n'a besoin que d'être renseignée et éclairée. La surveillance de l'autorité publique n'est pas inutile pour conserver l'exploitation forestière: il ne suffi pas de défendre le défrichement, la société gagnerait à assurer la conservation du matériel-bois, car l'intérêt privé est loin d'y suffire. Il est à regretter qu'en l'état de nos mœurs cette conservation ne puisse être exercée que pour les forêts de l'État et des communes: celle-ci s'élève dès lors à la hauteur d'un devoir social. 


\section{A P P E N D I E}

\section{NOTE A}

\section{Emploi des intérêts simples dans les calculs concernant les forêts.}

Cotta, célèbre forestier allemand, s'est donné la peine de calculer des tarits pour la loi des intérêts simples et de les établir sur des formules concues par analogie des intérêts composés. C'est ainsi que le capital futur $\mathrm{M}$ formé au bout de $n$ ans à intérêts simples est :

$$
\mathrm{M}=\mathrm{P}(1+n t) \quad(\text { tarif } I)
$$

La valeur actuelle $\mathrm{P}$ d'un capital $\mathrm{M}$ ramené à l'actualité est :

$$
\mathrm{P}=\frac{\mathrm{M}}{\mathrm{l}+n t}
$$

Le fonds générateur $F$ d'un revenu $R$ se produisant tous les $n$ ans à intérêts simples, est :

$$
\mathrm{F}=\mathrm{R} \frac{\mathrm{l}}{n t}
$$

Outre les motifs que nous arons donnés $n^{\circ} 18$, pour faire

( $\left.{ }^{1}\right)$ Ces tarifs à intérêts simples ont été publiés, en France, par II. de Salomon dans son Traité d'aménagement, 3 vol. in-8 ${ }^{\circ}$, Paris 1837. 
écarter absolument tout calcul fondé sur l'intérêt simple, il est utile de remarquer que son emploi, ainsi généralisé par une simple analogie de formules, conduit à de véritables absurdités.

Voici, par exemple, 40 hectares de taillis aménagés de 1 à 40 ans et produisant tous les ans 1 hectare de coupe valant $1,000 \mathrm{fr}$.

La valeur de cette propriété, pour celui qui voudrait faire emploi de son argent, à un taux $t$ (valeur relative), sera :

$$
1000 \times \frac{1}{t}
$$

Un seul hectare rasé est un fonds gẻnérateur d'un revenu de 1,000 fr. tous les 40 ans et vaudrait à intérèts simples, immédiatement après la coupe :

$$
1000 \times \frac{1}{40 . t}
$$

De sorte que 40 hectares rasés et mus vaudraient :

$$
1000 \times \frac{40}{40 . t}=1000 \times \frac{1}{t}
$$

Ces 40 hectares exploités auraient identiquement la même valeur que 40 hectares aménagés et sur lesquels il $\mathrm{y}$ a un matériel-bois de 1 à 40 ans ! Est-ce assez absurde?

C'est cependant le résultat que fournissent les tarifs de Cotta, publiés en France, en 1837, par M. de Salomon. On voit, en effet, que à 4 0/0 par exemple:

Les 40 hectares de taillis aménagés valent

$$
1000 \times 25=25000 \mathrm{fr} \text {. }
$$

Les 40 hectares de taillis exploités et rasés valent :

$$
40 \times 1000 \times 0,625=25000 \text { fr. }(\text { tarif III })
$$

On a peine à comprendre qu'un homme de mérite comme Cotta se soit laissé entraîner à une conception de cette nature. Il est vrai de dire que dans son Instruction sur la valeur des forêts publiée en 1827, par ordre de l'administration saxonne pour servir de règlement dans les estimations judiciaires du sol forestier, Cotta ajoutait 
que la vérité se trouvait probablement entre l'intérêt simple et l'intérêt composé; il s'était donné la peine de calculer, sous le nom d'intérêts moyens, et pour tous ses tarifs, un facteur intermédiaire obtenu par la moyenne arithmétique entre le facteur des intérêts simples et celui des intérêts composés. Que dire de cette vérité qui est ainsi composée d'erreur? 


\section{NOTE B}

\section{Définition de l'exploitation aménagée.}

La notion économique de l'aménagement paraît avoir été inconnue des anciens forestiers qui la mettaient cependant en pratique, sous le nom de mise en règle, ou en coupes réglées dans les anciennes réformations des forêts. Mais l'aménagement a, dans leurs écrits, les sens les plus divers et les plus singuliers.

Massé. - Aménager signifie débiter un arbre en bois de charpente ou autrement. Il est synonyme de exploiter. (Dictionnaire portatif des eaux et forêts, Paris 1766.)

Chailland. - L'aménagement consiste dans le recépage des bois abroutis et le repeuplement des places vaines et vagues. (Dictionnaire raisonné des eaux et forêts, $\mathrm{Pa}-$ ris, 1769.)

L'aménagement consiste dans le recépage des bois abroutis et dans lo repeuplement des places vaines et vagues et, en général, dans tout ce qui peut ètre l'objet de l'amélioration des bois. (Commentaire sur l'ordonnance des eaux et forêts de 1669, publié par Debure, note 3, Paris 1772.)

Les forestiers modernes ont seuls donné à l'anıénagement son véritable sens économique et en ont marqué le caractère réel par la condition que les produits de l'exploitation deviennent annuels, caractère conforme à l'étymologie et au but à atteindre. Aussi, les auteurs les plus nombreux et parmi lesquels nous ne citerons que les principaux, ne manquent pas de caractériser leurs définitions par cette condition du produit annuel.

Baudrillart. - C'est l'art de diviser une forèt en coupes successives ou de régler l'étendue ou l'âge des coupes annuelles, de manière à assurer une succession constante 
de produits pour le plus grand intérèt de la conservation de la forêt, de la consonmation en général et du propriétaire. (Annuaire forestier de 1S1\% - Dictionn. des eaux el forêts, Paris 1823. Vo Aménagement.)

Dralet. - L'aménagement est l'art de déterminer les parties qui doivent être coupées chaque année de manière à procurer les produits les plus avantageux tant au propriétaire actuel qu'à ses successeurs. (Traité de l'aménagement des forêts. Paris 1812.)

De Salonon. - Aménager une forêt, c'est déterminer la possibilité ou régler son exploitation de manière à assurer anmuellement une succession constante et égale des meilleurs produits possibles. (Traité de l'aménagement des forêts, Paris 1837, p. 5. .)

Norrot-Bonnet. - L'aménagement peut être défini : Un travail dont l'objet est de constituer la production forestière de manière à assurer la renaissance anmuelle d'un revenu constamment égal. (Théorie de l'cuénagement des forêts, Paris 1842, p. 1.)

'TASSY. - L'aménagement est un travail qui consiste à régler l'exploitation d'une forêt de façon qu'elle fournisse un rapport anmuel aussi soutenu et anssi avantageux que possible. (Etudes sur l'aménagement des forêts, $3^{\circ}$ édition, 1887, p. 1.)

M. Tassy ajoute qu'il a cr'u devoir maintenir cette définition quoiqu'elle diffère de celle du Cours de culture, parce que la condition du rapport annuel n'est pas comprise dans la définition de ce Cours : elle est pourtant, dit-il, caractéristique de l'aménagement. (Note de la deuxième édition, 187\%.)

Il est vrai que d'autres auteurs se sont écartés de cette manière de comprendre l'aménagement et en ont tellementélargi le sens, qu'il est impossible de trouver dans leurs définitions un caractère précis du but assigné à l'exploitation aménagée.

T'homas. - L'art des aménagements consiste à multiplier les revenus en assurant leur continuité et à exploiter les hois en temps utile, c'est-à-dire, avant qu'ils entrent en décrépitude. (Traité général de statistique, culture et exploitation des bois, Paris 1840, p. 205.) 
Lorextzet PARADE. - Opération qui consiste à régler, ponr une on plusieur's rérolutions, le mode de culture d'une forêt, ainsi que la marche et la quotité de ses exploitations. (Cours de culture des bois, 6o édit., Paris 1883, p. 391.)

Narquetre. - L'aménagement est une opération qui consiste à régler, pour une ou plusieur's rérolutions, l'exploitation d'une forèt, dans le plus grand intérèt du propriétaire et de la consommation. (Cours d'aménagement des forêts, Nancy 1860, p. 1.)

BRorlirard. - L'aménagement est l'art de régler le traitement et l'exploitation des forèts. (Cours d'aménagement des forets, 1878, p. 21.)

A. Rousset. - L'aménagement est la méthode à suivre pour l'exploitation des bois. (Culture et exploitation des arbres, Valence 1882, p. 412.)

B. DE LA Grye. - Aménager une forèt, c'est arrêter d'une manière définitive le mode de traitement qui doit luj être appliqué, la quotité et l'ordre des exploitations. (Régime forestice, Paris 1883, p. 2i.)

Reuss. - L'aménagement des forêts est l'art de réglementer l'exploitation des forêts en rue des besoins de l'homme. (Cours d'aménagement, autogr'. 1886, p. 1.)

Les auteurs allemands offrent dans leurs définitions la mêrne indécision que les forestiers français : ceux-ci, s’ils donnent à l'aménagement des sens différents, ont au moins le mérite de se servir tous du mème mot, tandis qu'en Allemagne les termes employés sont les plus divers et, de plus, les auteurs se servent de ces termes en leur attribuant des sens différents. Cependant, dans le dernier état de la science forestière allemande, tel qu'il résulte du livre de Carl Heyer dont la troisième édition date de $1883\left({ }^{1}\right)$, l'aménagement (Waldertrags-Regelung, littéralement règlement du revenu des forêts) est une partie de l'organisation et de la mise en ordre convenable de l'exploitation

(1) Carl Hever. - Die Waldertrags-Regelung; 3 e édit. rerue par Gustav llever, 1 rol. in-8 $8^{\circ}$, Leipzis 1883. 
(Forstwirthschafts - Einrichtung; Forstbetriebseinrichtung; Betriebsregulinung).

Cette organisation de la forêt comprend, suivant C. Heyer :

$1^{\circ}$ Le partage en divisions de surveillance et de gestion ;

$2^{\circ}$ La formation du personnel technique et la répartition de ses attributions;

$3^{\circ}$ Le choix du mode de traitement, des essences, des procédés de régénération, de l'âge d'exploitation et le programme des travaux de repeuplement;

$4^{\circ}$ Le comptage et le cubage des bois sur pied;

$5^{\circ}$ La mise en revenu annuel ou, en d'autres termes, la réglementation du revenu dans le présent et dans l'avenir (Waldertrags-Regelung) ce qui est, dit l'auteur, l'aménagement des forestiers français.

On voit que les forestiers allemands comprennent par cet ensemble d'opérations quelque chose d'analogue à ce que nos anciennes maîtrises pratiquaient sous le nom de réformation ou mise en ordre de la forêt. Nos anciennes réformations, après s'être occupées, en effet, du bornage, du règlement des anticipations, de la division en séries et du traitement, prescrivaient ensuite la mise en règle des coupes qui exprime l'idée de notre aménagement pour ainsi dire par le même mot que les auteurs allemands.

C. Heyer ajoute que ce règlement du revenu annuel, cette mise en revenu (Waldertrags-Regehung) a été exprimée et comprise par d'autres forestiers sous les dénominations suivantes:

$1^{\circ}$ Aménagement, par les auteurs français;

$2^{\circ}$ Forstsystemisimung, par les auteurs autrichiens;

$3^{\circ}$ Forstlaxation, par Hennert, 179]. ; G. L. Hartig, 1795 ;

H. Cotta, 1804; Pfeil, 1833 et 1858.

$4^{\circ}$ Forstabschxtsung, par Ilundeshagen, 1824.

$5^{\circ}$ Forsturganisation, par André, 1823. 
$6^{\circ}$ Forstbetriebsregulirung, par Klipstein, 1823; Wedekind, 1834 ; Karl, 1838 ; Grebe, 1867.

7o Forsteinrichtung, ou Forstbetriebseinrichtung, ou Forstwirthschaftseinrichtung, par Beckmann, 1759 ; Ettelt, 1768 ; Cotta, 1820; L. F. Hartig, 1825.

Ce résumé des indications fournies par un professeur justement estimé montre qu'en Allemagne, on comprend l’aménagement comme une opération caractérisée par la condition du revenu annuel et comme une partie de ce que nos prédécesseurs pratiquaient sous le nom de réformation des forêts. C'est la même définition que nous avons adoptée dans notre livre avec MM. Baudrillart, Dralet, de Salomon, Noirot-Bonnet, Tassy, etc. Il en est de même, enfin, en Italie, où l'opération est pratiquée sous le nom d'Assestamento forestale (F. Piccioli, Elementi di tassazione ed assestamento forestale, Florence 1881).

Il n'est pas inutile de remarquer que, dans le langage du Droit. le mot aménagement a un sens beaucoup plus large ; quand l'article 590 du Code civil impose, par exemple, à l'usufruitiel' de suivre l'aménagement de l'ancien propriétaire, ce mot comprend, à la fois, le traitement cultural, le genre d'exploitation, l'âge des coupes et toutes les dispositions adoptées pour la conduite de l'immeuble. La loi assigne, dans ce cas, à l'aménagement de la forêt le même sens qu'à celui d'une ferme ou d'une industrie, et on comprend qu'il ne saurait en être autrement quand il s'agit d'assigner une règle à la jouissance. C'est le propre de chaque science d'avoir son langage spécial adopté au but qu'elle poursuit.

L'économie forestière devait également emprunter sa définition au caractère de l'exploitation et la préciser exactement d'une manière conforme à son objet. 


\title{
TABLE DES MATIÈRES
}

Première Partie

\section{NOTIONS PRÉLIMINAIRES}

\author{
CHAPITRE I ${ }^{\circ}$
}

\section{CAUSERIE FORESTIËRE}

Nos 1. - Avantages généranx des placements en forêts : sécurité.... 11

2. - Facilité de leur gestion .................. 12

3. - Amélioration graduelle du revenu et de la valeur capitale. 13

4. - Taux du placement, motifs de sa morlération.......... 17

э. - Avantages particuliers. - Combinaisons d'exploitation.... 19

6. - La forêt, caisse de dépôt et d'épar.gne............ 21

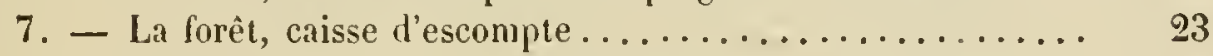

8. - Immeuble agréablc et intéressant............. 24

9. - Objections. - Faut-il labiter la campagne pour être propriétaire de bois ?.................. פכ

10. - Faut-il être riche pour en acheter ?........... 27

1I. - La propriété forestière est-elle assez protégée ?........ 29

12. - Il fiut s'y connaître pour les administrer .......... 31

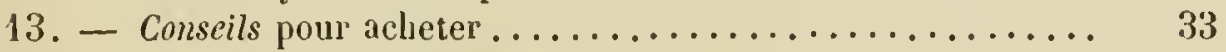

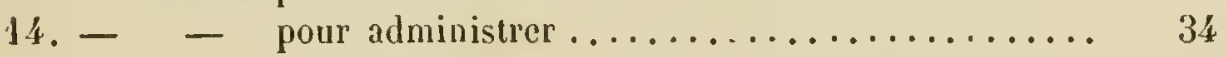

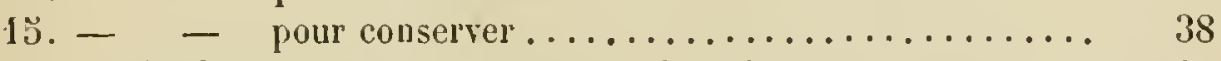

16. - Quelques mots pour les agents forestiers.......... \&I

\section{CIIAPITRE II}

\section{THÉORIE DE L'INTÉRE்T}

Article $1 \mathrm{er}$.

\section{Générnlités.}

17. - Loyer des capitaux ou intérèt............... 43

18. - Trois lois de formation des capitaux ........... 4; 
Nos 19. - Deux questions fondamentales dans chaque loi de formation : 10 relation entre le capital initial et le capital futur ; $2^{\circ}$ relation entre le capital initial et son loyer : quatre problèmes et quatre tarils..............

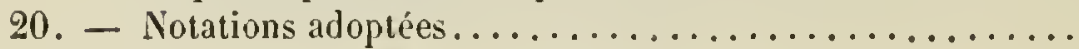

Article 2.

\section{Loi de formation par l'intérèt composé.}

21. - Premier probleme. - Capital futur II en fonction du ca-

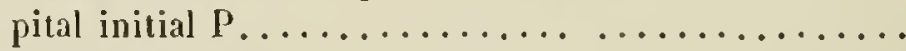

22. - Denxième problème. - Revenu on intérêt $\mathrm{R}$ en fonction

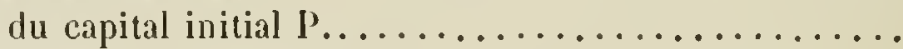

23. - Tarif I, facteur de sommation et facteur d'intérêt......

21. - Troisième problème. - Valeur actuelle $\mathrm{P}$ d'un capital futur $\|$ en fonction de ce capital.................

25. - Tarif II, facteur de réduction à l'actualité. - Escomple .

26. - Quatrième problème. - Valeur du capital générateur $\mathrm{F}$ en lonction du revenu $\mathrm{R}$...................

27. - Tarif III, lacteur ou deniers de génération d'un revenu

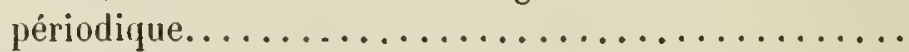

28. - Observations sur l'emploi de cette lormule...........

29. - Problèmes dérivés. - Trois problèmes déduits de la relation entre P (capital initial) el II (capital futur)......

30. - Trois problèmes déduits de la relation entre F (fonds gé-

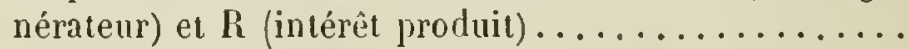

31. - Observations sur la valeur du capital initial et du fonds

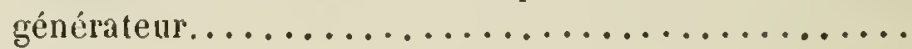

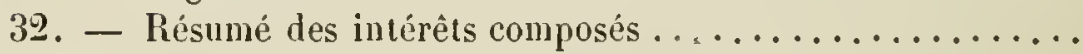

Article 3.

\section{Loi le formation par les annuités.}

33. - Premier problème. - Montant ou valeur future de $n$ an-

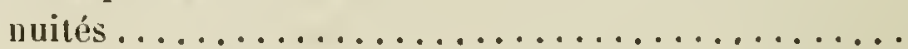

34. - Deuxième problème. - Inutilité de calculer le loyer produit

35. - Troisième problème. - Principal ou valeur actuelle génératrice de $n$ annuités....................

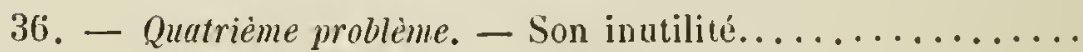

37. - Questions dérivées du problème du montant : économie annuelle, feuille, rente annuelle équivalente, valeur des

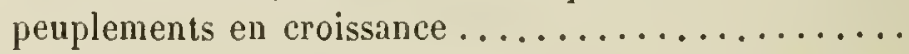

38. - Nombre d'années nécessaires pour former un capital par économies annuelles.....................

39. - Taux auquel les économies ont fonctionné .........

40. - Questions dérivées du problème de la delte: anortissement

41. - Temps nécessaire à l’amortissement d'ıne dette....... 84

42. - Taux auquel la dette a élé consentie ............ 85

43. - Riésumé des formules................. 87 
Article 4.

\section{Loi de formation par les annuités périodiquen.}

Nos 44. - Problème du montant d'un certain nombre $n$ d'annuités périodiques........................ 88

49. - Problème du principal de $n$ annuités............ 89

46. - Capital générateur d’annuités périodiques.......... 89

47. - Résumé des formules.................... 91

Article כै.

\section{Usufruit.}

48. - Calcul de la valeur de l'usulruit : $1^{\circ}$ d'une rente annuelle ; go d'une rente périodique................. 91

49. - Valeur de la nue propriété dans ces deux cas........ 92

50. - Usufruits concédés sous condition suspensive ........ 93

51. — Formule générale des estimations de forêts......... . 94

52. - Procédés équivalents mais plus compliqués assignant des valeurs distinctes au fonds et à la superficie; méthode de l'escompte....................... 97

53. - Résumé des formules..................... 100

ร̌4. - Observation........................ 100

\section{Deuxième Partie}

\section{CONCEPTION THÉORIQUE DE L'EXPLOITATION FORESTIÈRE}

CHAPITRE Ier

\section{CONSTITUTION DE L'EXPLOITATION FORESTIERE}

Article 1 er.

\section{Éléments constitutifs de l'exploitation.}

รั. - Définition de la forêt.................. 10马ّ

50. - Éléments constitutifs de l'exploitation : l'arbre isolé et le peuplement d'un seul îge................. 108

57. - Disposition de ces éléments dans l'exploitation fores. tière : trois types.................... 110

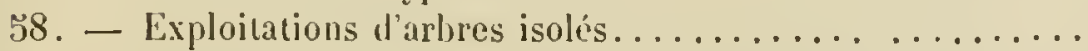

59. - - Exploitations de peuplements d'un senl àge .........

60. - Exploitations mixtes d'arbres isolís et de peuplements d'un seul ìge ....................... 116

61. - Sens du mot peuplement en sylviculture............ 118

62. - Modes de perception de la récolte: trois systimes de r:oupes ........................ 119

63. - Trois modes d'assiette des coupes, leur importance.... 
Article 2.

\section{Capital d'exploitation.}

$\S 1$ er. - Distinctions entre les exploitations.

Nos 64. - Constitution de l'exploitation forestière : travail, agents

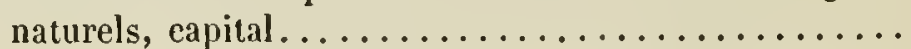

65. - Différentes espèces d'exploitations : périodique, aména-

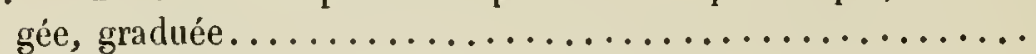

66. - Exploitations simples et composées .............

\section{$\S 2 .-$ Exploitations simples.}

67. - Exploitations périodiques d'arbres, matériel-bois .......

68. - - _ - de peuplements d'un seul âge...

69. - - - mixtes d'arbres et de peuple-

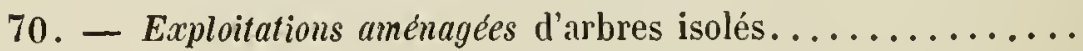

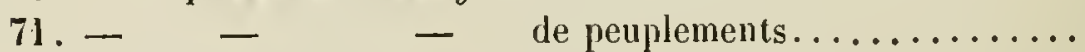

72. - - _ _ - mixtes d'arbres et de peuplements

73. - Exploitations graduées : leur constitution et leur matériel.

74. - Utilité de ces exploitations pour les petites forêts .....

75. - Combinaisons des forêts jardinées pour l'aménagement des

76. - $\quad$ pour les domaines moyens.......... 146

§3. - Exploitations composées.

77. - Exploitations périodiques d'arbres isolés ............

78. - - - $\quad$ - de peuplements d'un seul âge...

79. - - - - mixtes d'arbres et de peuplements

80. - Remarque sur l'effet de l'augmentation du matériel-bois.

81. - Exploitations aménagées : arbres isolés .............

82. - _ _ _ forèts de peuplements d'un seul

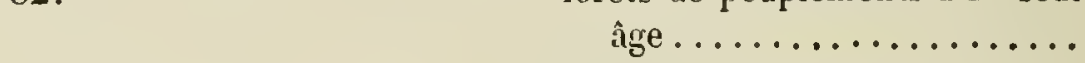
159

83. - $\quad$ - $\quad$ - forèts mixtes d'arbres et de peuplements............. 161

84. - Exploitations graduées : combinaisons diverses........

\section{\$4. - Observations.}

85. - Remarques générales sur le eapital d'exploitation des fo-

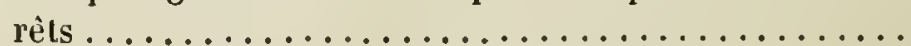

86. - Comparaisons avec les exploitations agricoles........

87. - Utilité de la notion du capital d'exploitation .........

88. - Portée de la conception théorique; forêt normale .....

\section{CHAPITRE II}

\section{AGE D'EXPLOITATION}

89. - But de l'exploitation. - Révolution ............ 
No8 90. - Problèmes sur l'âge d'exploitation. - Exploitabilité....

91. - Remarques sur ces problèmes................. 179

92. - Définition de la futaie ................... 182

93. - Exploitation intensive et extensive............. 183

94. - Comparaison avee les exploitations agricoles......... 183

9ั. - Rparyne nécessaire pour rendre une exploitation intensive ............................... 187

96. - Conversion et transformation................. 189

\section{CIIAPITRE III}

\section{PRODUIT DE L'EXPLOITATION}

Article $1{ }^{\mathrm{er}}$.

\section{Produit en matière.}

$$
\S 1^{\mathrm{er}} \text {. - Production. }
$$

97. - Définition et mesure de la production ............ 191

98. - Calcul de la production annuclle ............. 192

99. - Cáleul de la production moyenne .............. 197

100. - Observations sur la production moyenne.......... 199

\section{§ 2. - Revenu.}

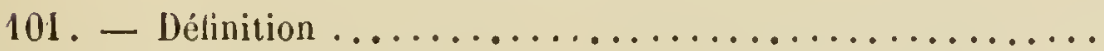

102. - Revenu annuel et production par année moyenne ..... 202

103. - Remarque sur la production comparée au revenu...... 20.

101. - Recherclıe de l'âge d'exploitation donnant la plus grande production en matière ; exploitation de peuplements.. 203

10ð. - Nème question pour l'arbre isolé ............. 208

\section{§ 3. - Possibilité.}

106. - Définition : trois formes de possibilité............

107. - Exemples de possibilités pour les divers modes d'exploitation.......................... 217

108. - Conditions auxquclles la possibilité doit satisfaire ..... 2.

109. - Origine el différents aspects de la possibilité ........ 223

110. - Mesure de la possibilité................. 226

111. - I'roduction possible : production de l'aménagement et

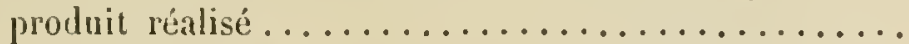

112. - La production résultant de la possibilité fournit-elle toujours la production réelle de la forèt ?.........

113. - Véthodes inventées pour fournir la production réelle

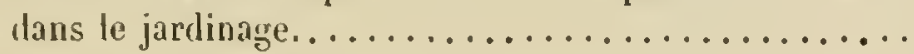




\section{Article 2.}

\section{L'roduit en argent.}

Nos 114. - Rente de la forêt, définition ............... 23 ב

Hכ̆. - Ia rente augmente arec l'àge d'exploitation ........ 237

116. - Conditions nécessaires pour cette augmentation de la rente.......................... 239

117. - Combien rapportent les forèts dans telle localité ?.... 240

118. - Fausse définition de la rente............... 2i1

119. - A quel àge faut-il régler l'exploitation pour obtenir la plus grande rente...$\ldots \ldots \ldots \ldots \ldots \ldots \ldots$

120. - Terme dexploitation convenant à l'État........... 121. - Utilité de la rente pour la gestion d'un domaine forestier .............................

\section{CHAPITRE IV}

\section{TAUX DE L'EXPLOITATION}

Article ler.

\section{Taux de placement.}

122. - Exploitations périodiques. - Taux de leur placement...

123. - Taux net du placement .................. 253

124. - Calcul du taux quand il y a les produits intermédiai-

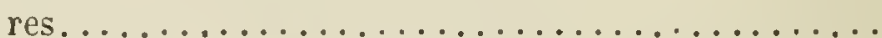

123. - Observations sur l'elfet des éclaircies.............

126. - Exploitations aménagées. - Formule du taux de place-

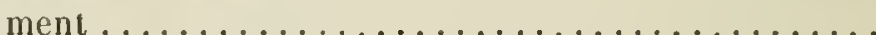

127. - Estimations du revenu et du matériel-bois .........

128. - Éraluation du sol......................

129. - Procédé erroné pour estimer les sols de forèts .......

130. - Effet de l'augmentation de l'outillage sur le taux du pla-

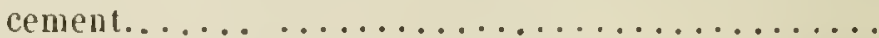

131. - Comparaisons du taux de placement des exploitations périodiques et anénagées faites au mème âge......

132. - Loi du taux des placements en forèts.............

133. - laiblesse du taux des exploitations à long terme.....

134. - Age d'exploitation correspondant à uu taux de placement déterminé ......................

13วั. - Age correspondant au maximum du taux de placement.. 136. - Conséquence économique de la diminution du taux de placement à mesure que l'àge d'exploitation aug-

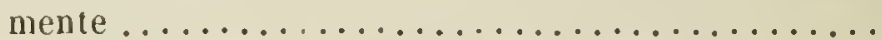

137. - Mesures légales d'encouratrement aux lutaies.........

138. - Rôle des forêts de l'État : faire des arbres .......... 283

139. - Conséquences de l'aliénation des forcts de l'État ..... 286 
ArTicLE 2.

\section{'ar de formation.}

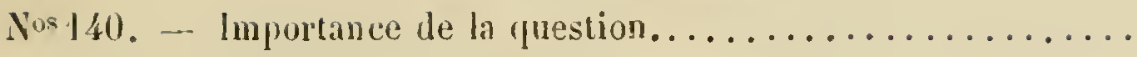

141. - Représentation les raleurs par l'intérèt composé pour l'arbre isolé..................... 240

142. - Idem pour le peuplement d'un seul igre...........

1 13. - Valeur des ricoltes acquises relatirenient au propriétaire

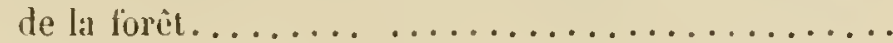

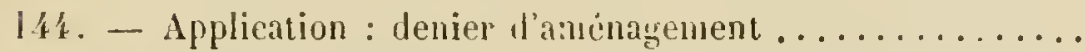

143. - Autre application : estimation l'une forêt exploitéc ì

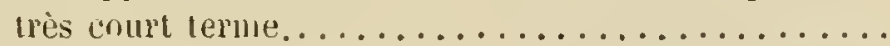

146. - Emploi spécial de l'estimation des récultes erga domimm en cas de dommages eausés à la propriẻté lirestière .

147. - Exemple du calcul pour un taillis sous futaie.......

148. - Ventes de récoltes sur pied; leur estimation ........

149. - Application à l'expropriation pour cause d'utiliti publique; cas d'un taillis ordinaire..............

Article 3.

\section{Comparaison agricole.}

130. - Kente du sol. - Sa définition, calcul de certains auteurs

lìl. - Fansse application ì la recherche de l'âge d'exploitation la plus avantageuse au point te vue du taux de placement.............................

152. - Fausse application a la comparaison des exploitations

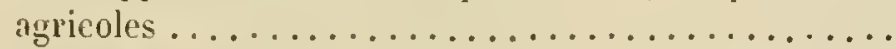

133. - Comparaison agricole. - Le tanx de placement est le moyen le plus simple de comparer les exploitations. .

15\%. - Conditions du problène..................

19\%. - Arantages des placements en lorcts ì capital égal.....

156. - Exploitations agricoles de moindre eapital. . . . . . . . .

13\%. - Conclusion.

306

\section{APPENDICE}

Note A. - Emploi des intérêts simples dans les calculs concernant

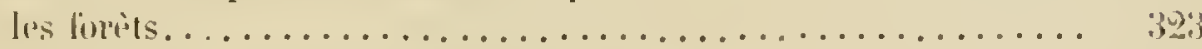

Note B. - Définition de l'exploitation ancenagre........... 3206

Table des matières........................

Nancy. - Impr. Nancéienne, 1, rue de la Pépinière. - Directeur : Plikson. 

Ensouchiment Sol

FIG. 1 .

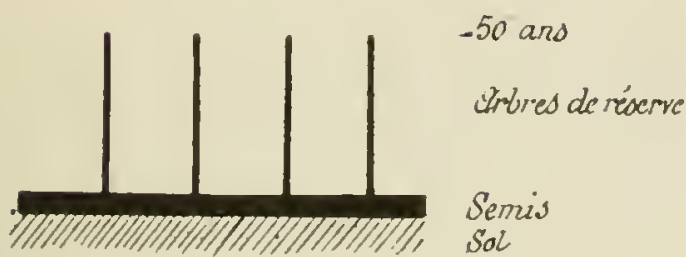

FIG. 3 .
Taillis

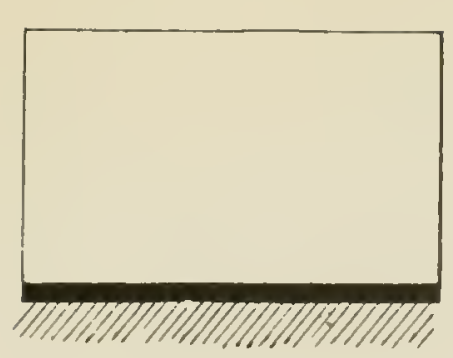

FIG. 2 .

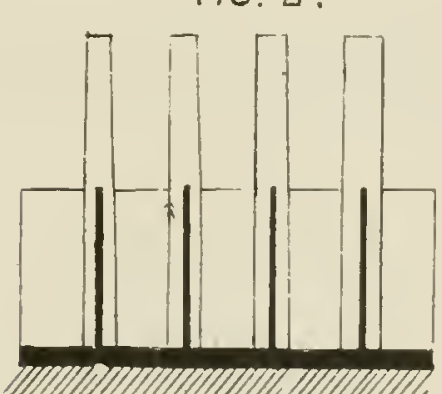

100 ans

arbes

50 ans

peuplement

Serris

Sol

Ensouchement Sol

penpent

FIG. 4.

30 ans

FIG. 5 .
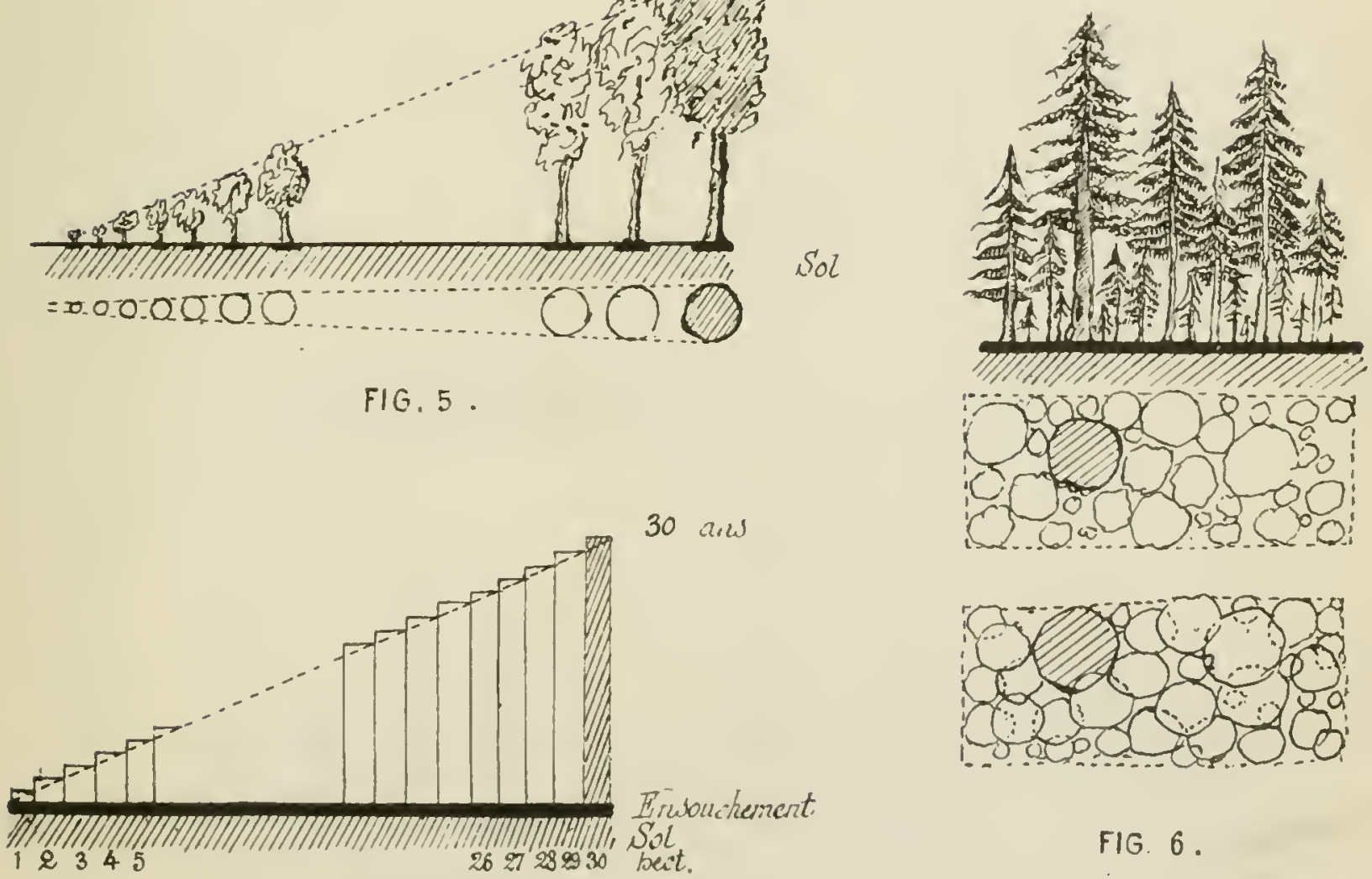

FIG. 6 . 


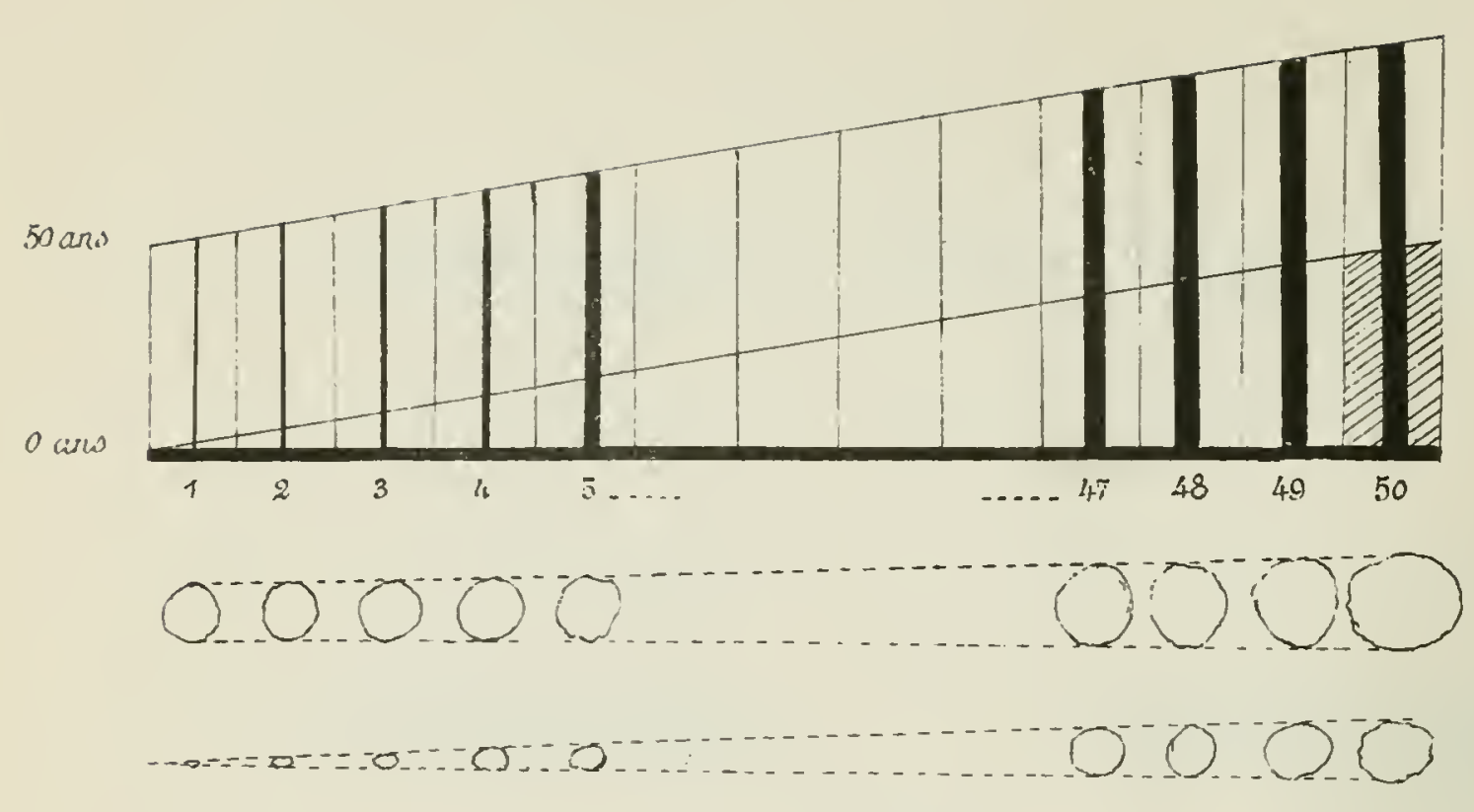

FIG. 8 .

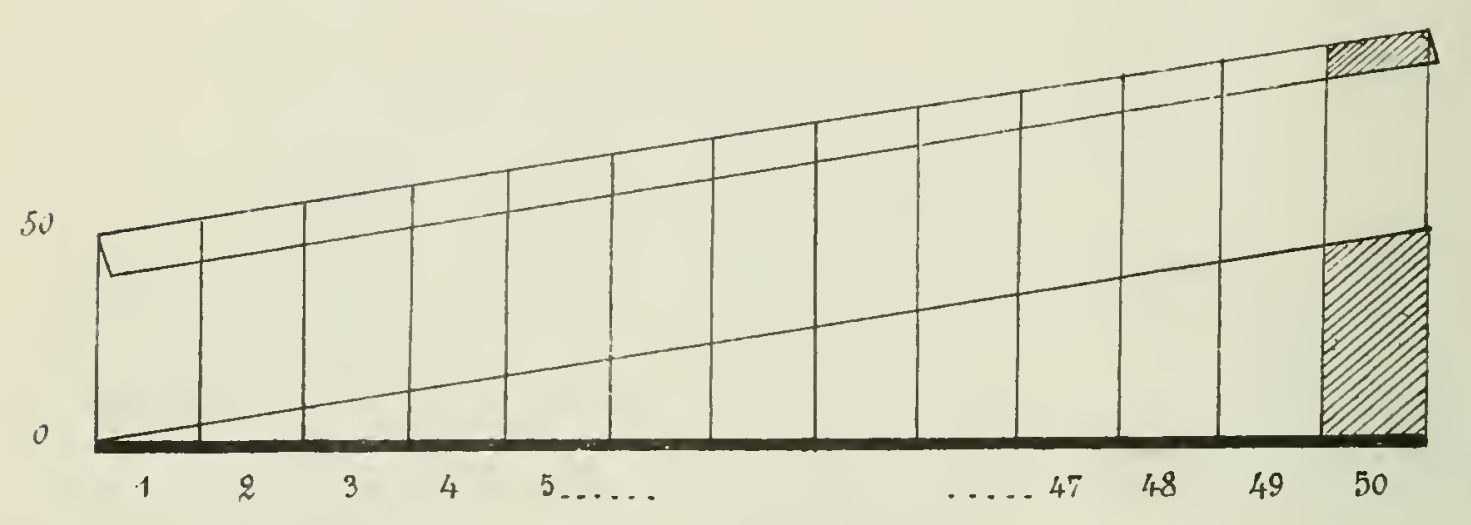

100 ans

FIG. 9 .

ligne des hectares

ligne des hectares

50 ans

100 ars

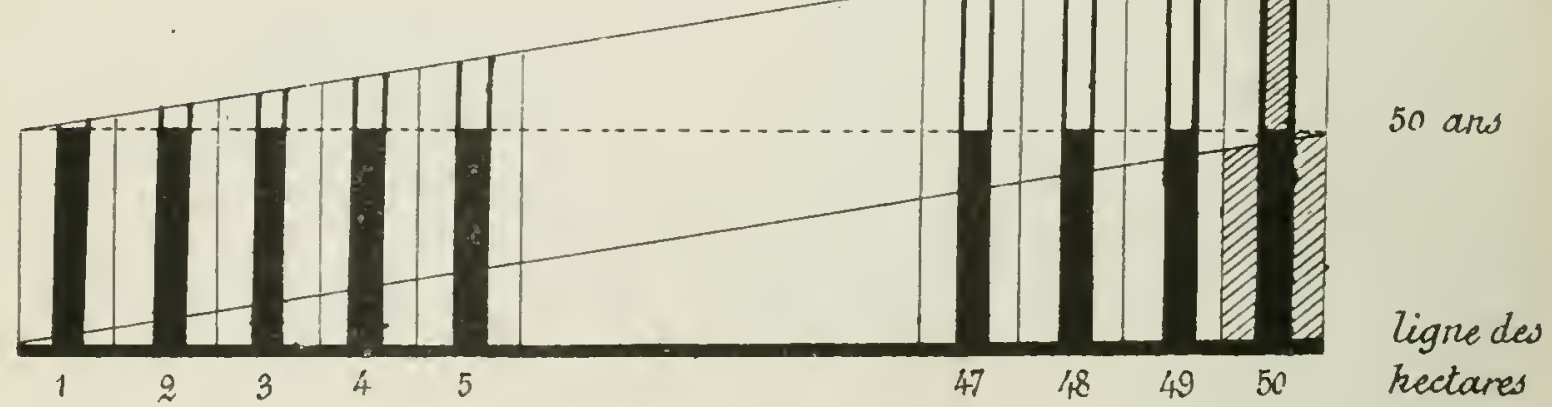

FIG.10. 
30 rins

$12345 \quad 5 \quad 6 \quad 7 \quad 3 \ldots \ldots$ 1
인
3

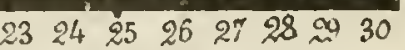

5

6

ligne des surfaces.

FIG.11.

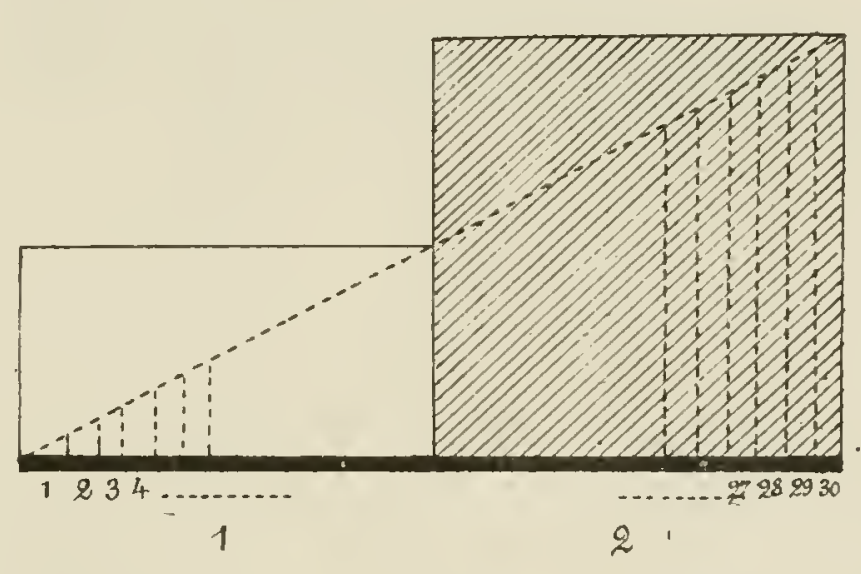

30 and

15 ans

FIG.12.

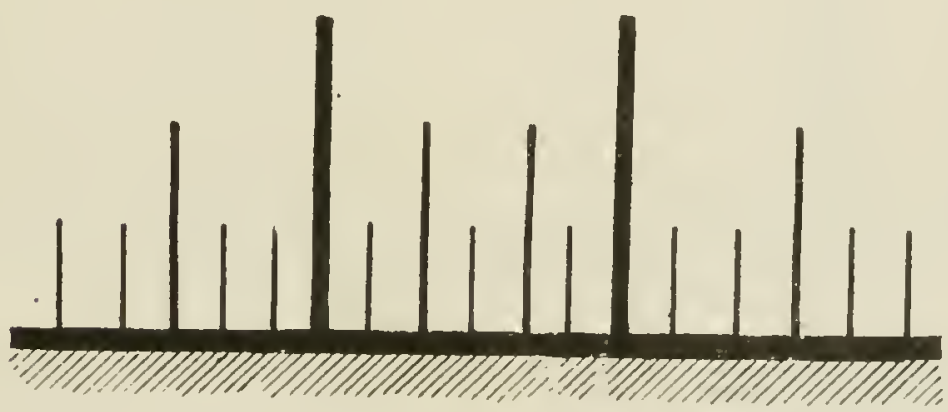

90 ans anciens

60 ans modernes

30 ans baliveaux.

Erwuckernent Sol

FIG. 13.

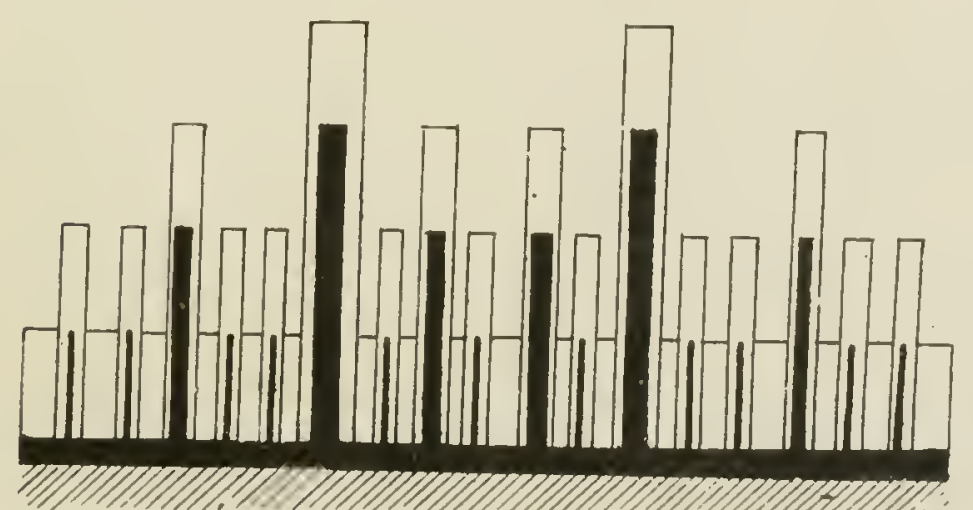

180 ans lis siviens

90 ans ancions

60 arw inoderpies

3.) ans baliverux et titillis

Erwouchement Sol. 


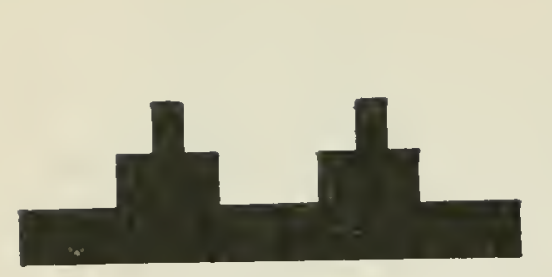

FIG. 15.

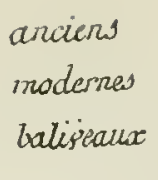

balisianx

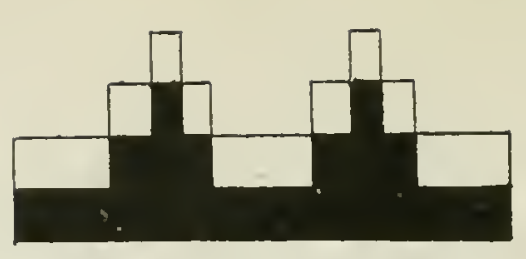

FIG 16 . bis ansiens anciens modernes baliveaus

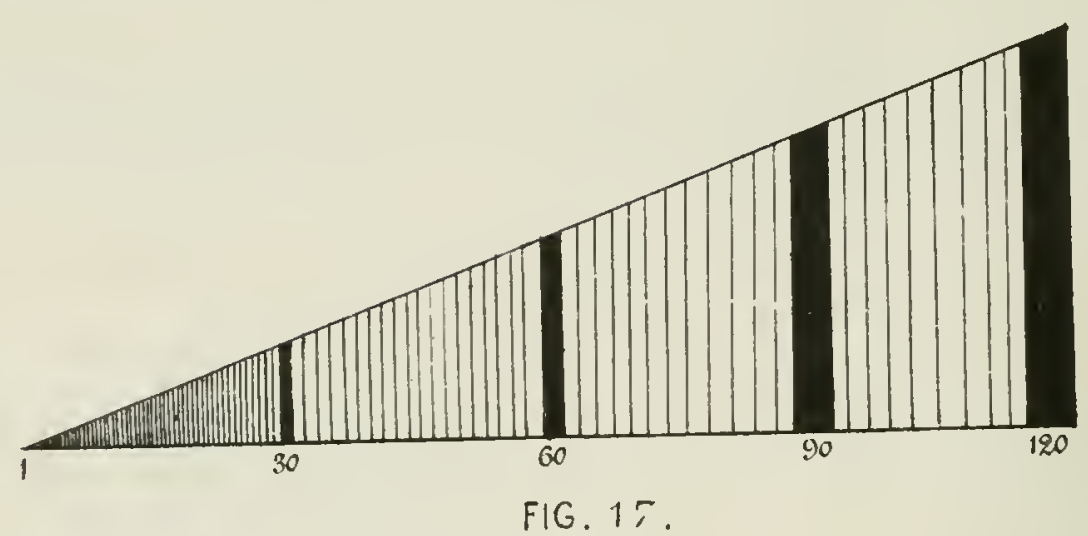

190 ano

120 ans bis anciens

90 ans anciens

60 ans modernes

60

90

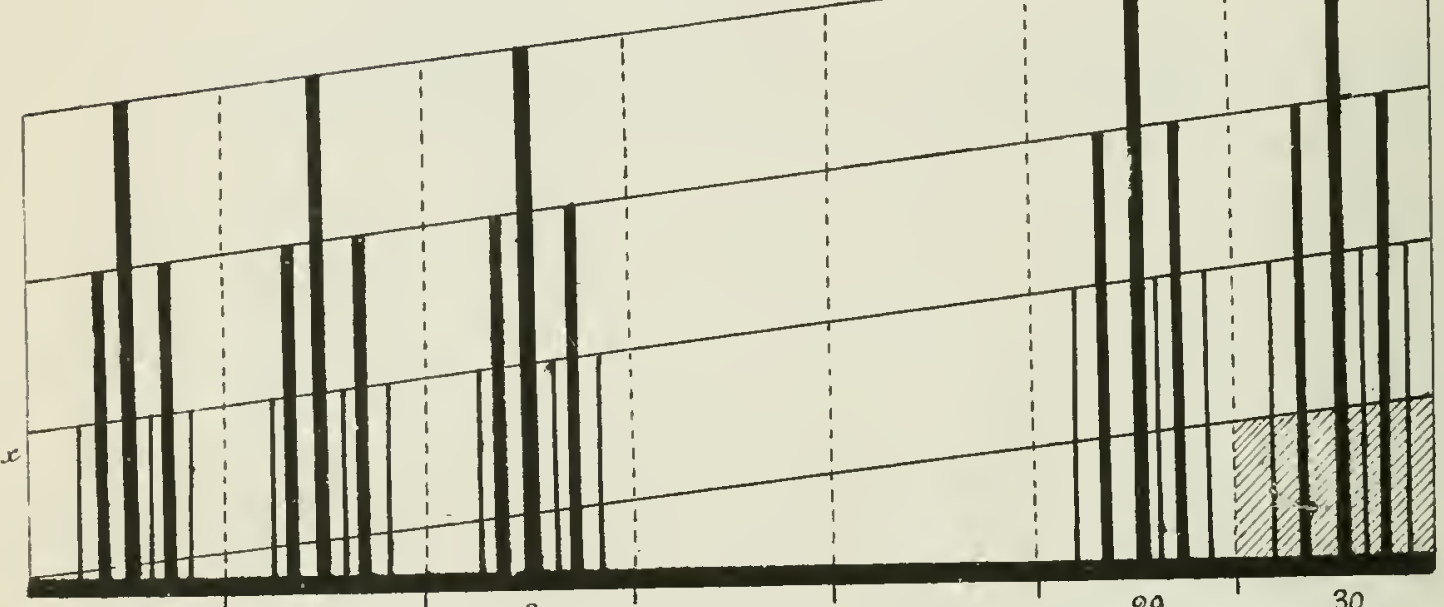

2

$$
3
$$

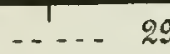

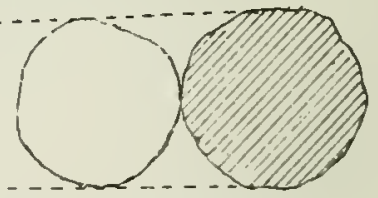

100 ans

91

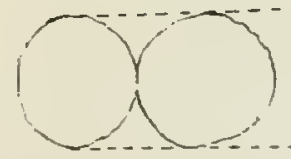

61

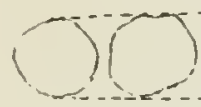

31
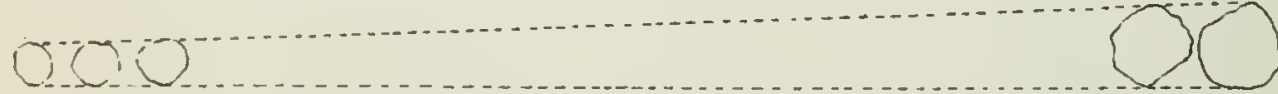

60

FIG. 18 . 


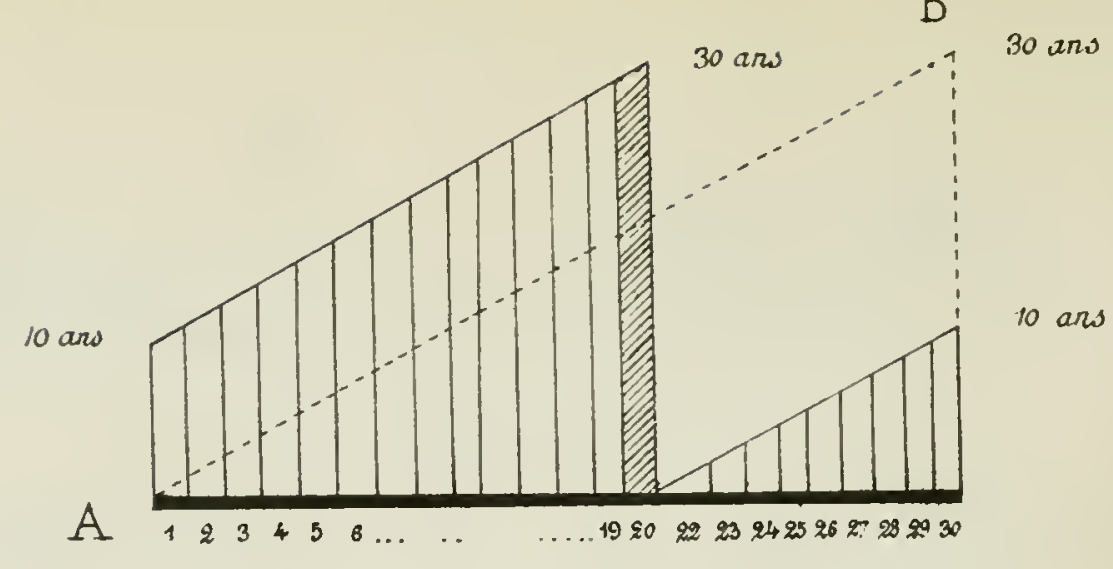

FIG.21.

90 cons

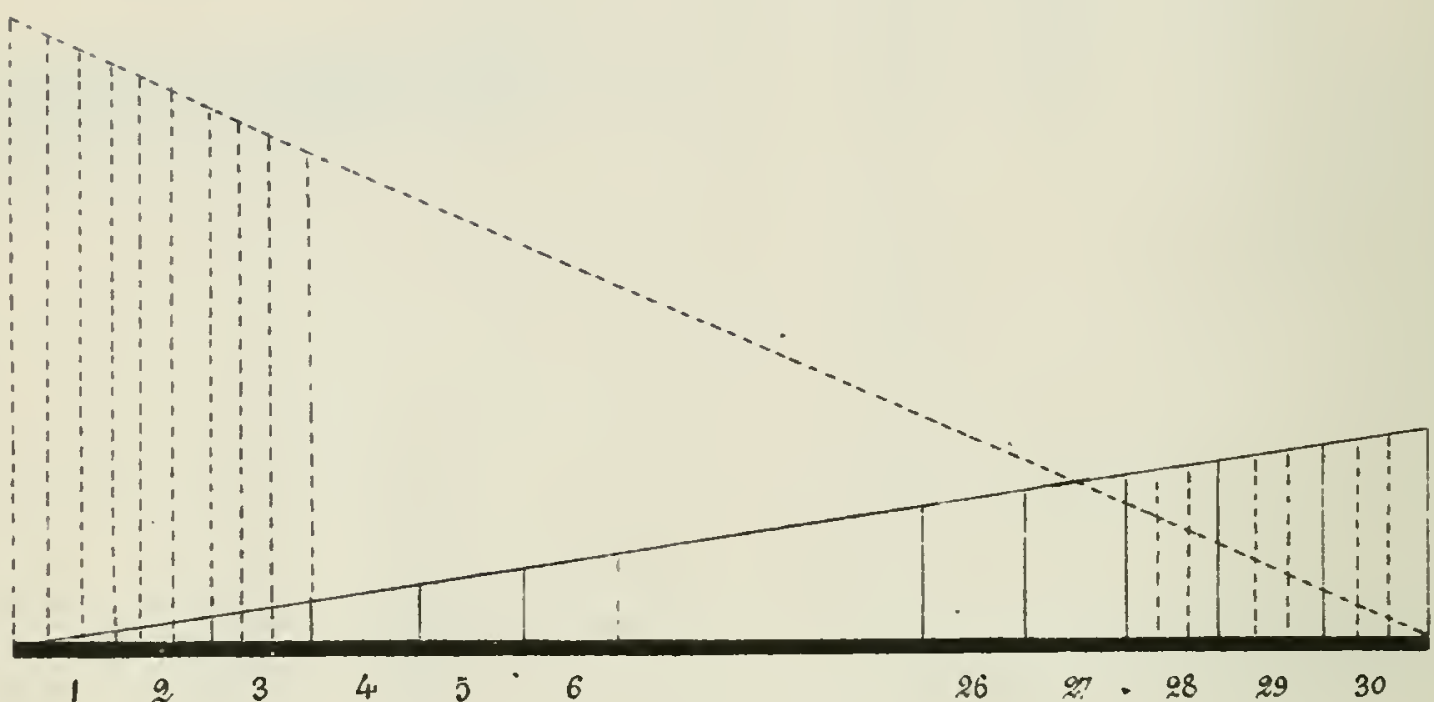

FIG 22.

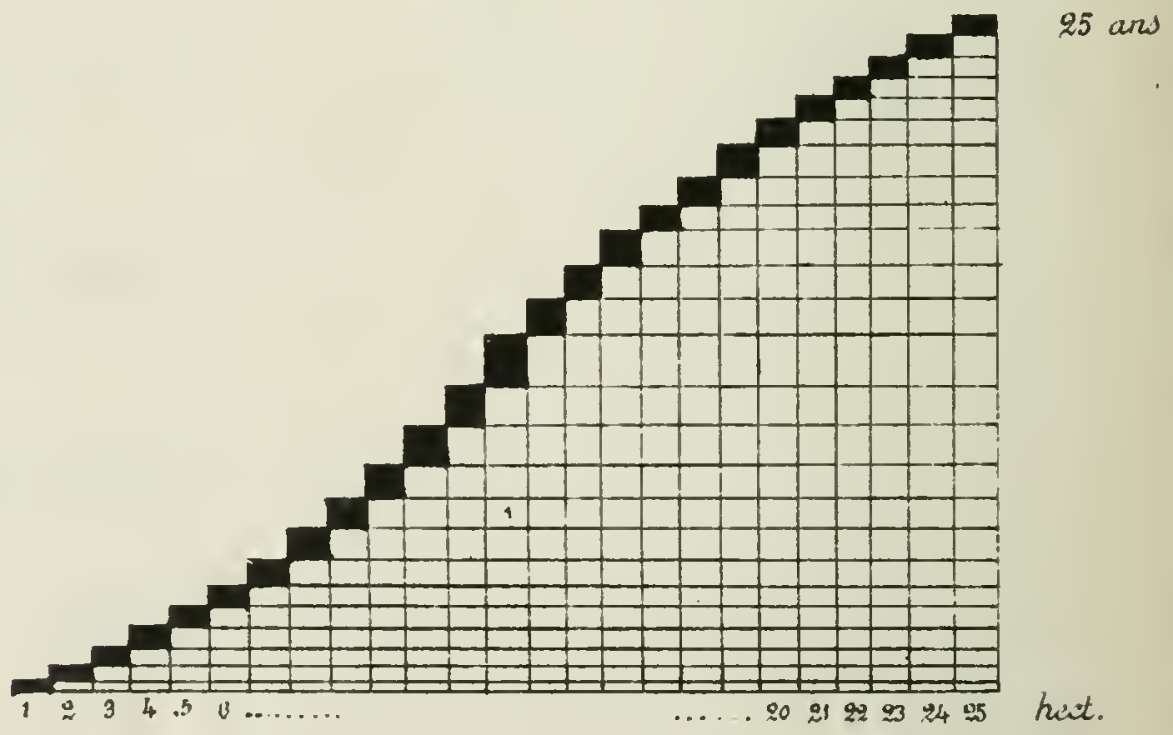

FI․ 23. 

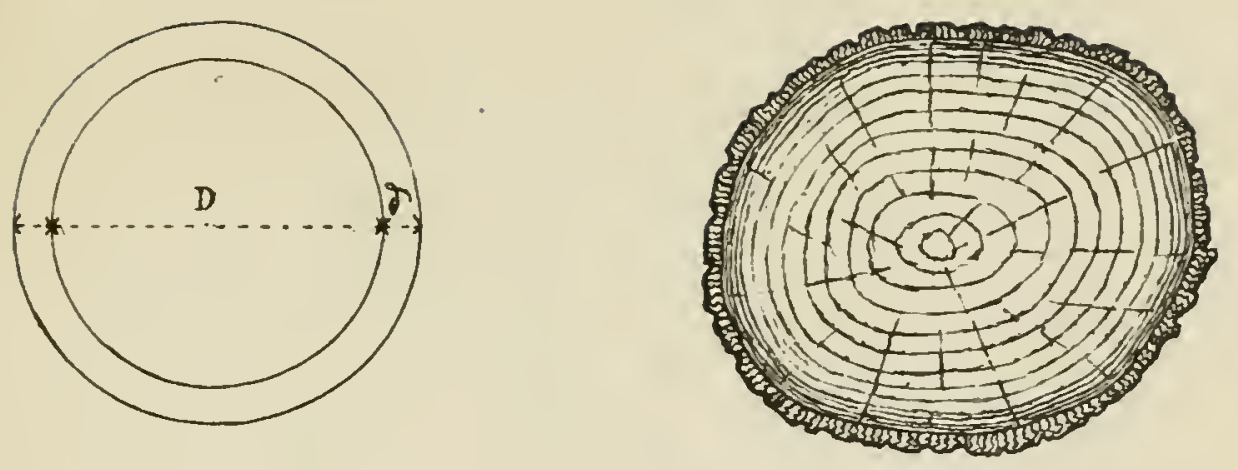

FIG 24

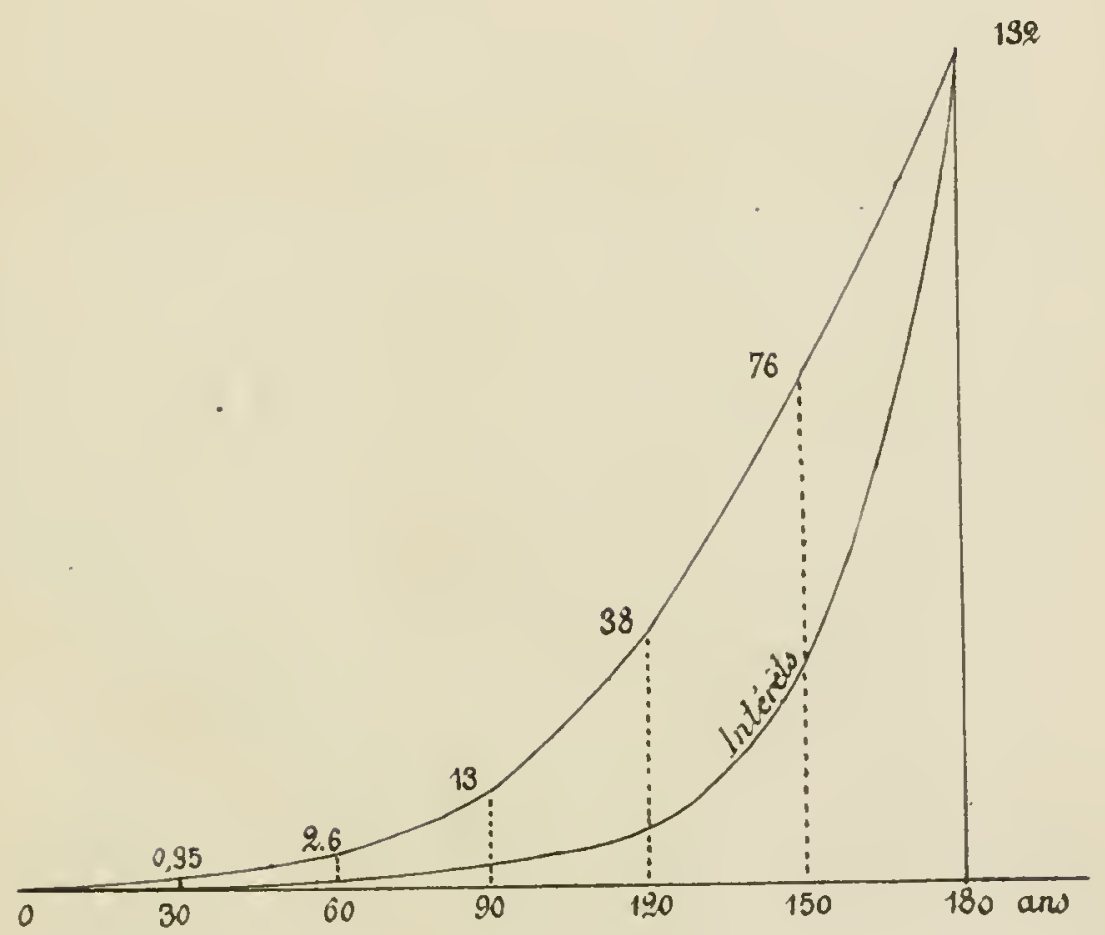

FIG. 25. 





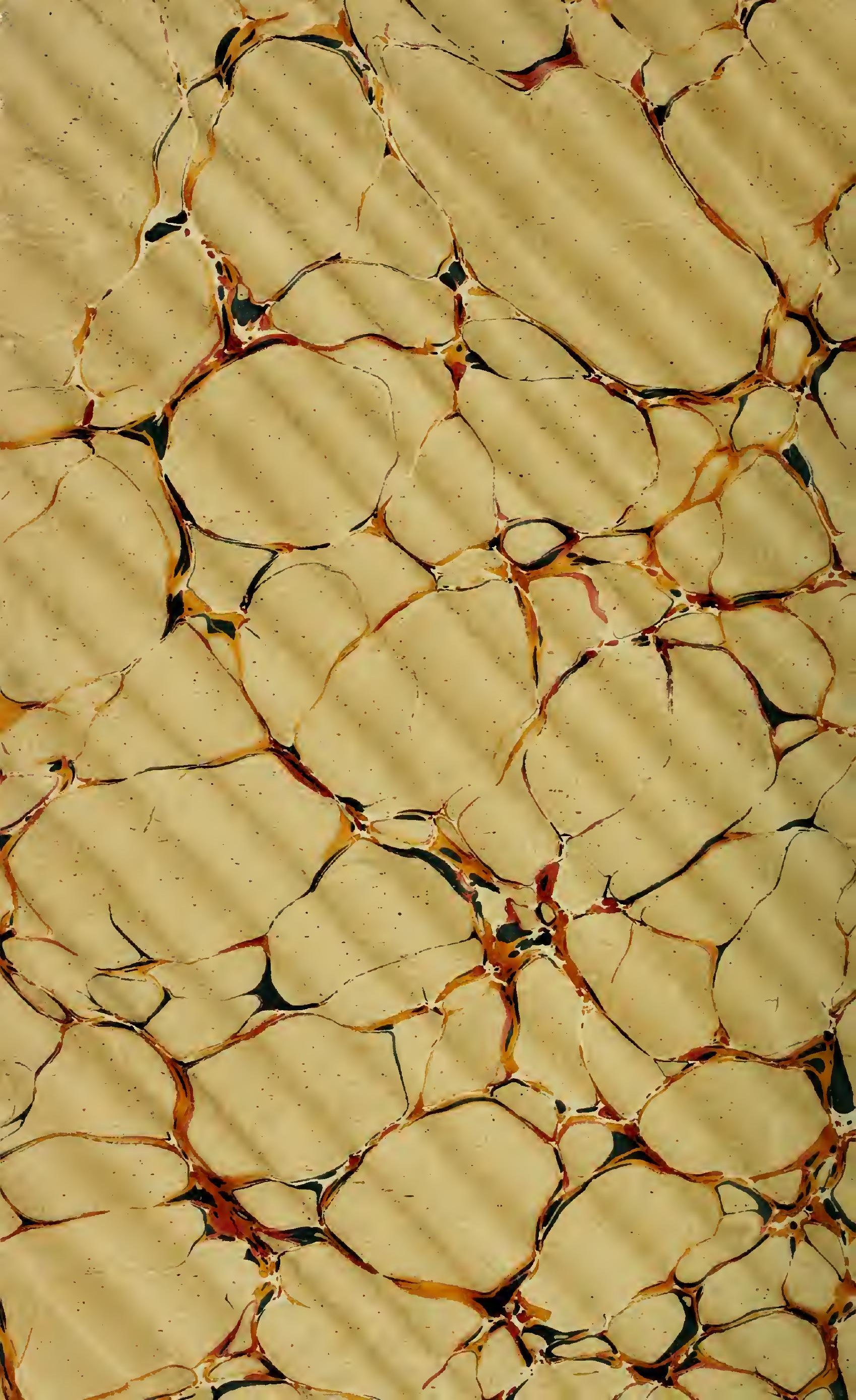




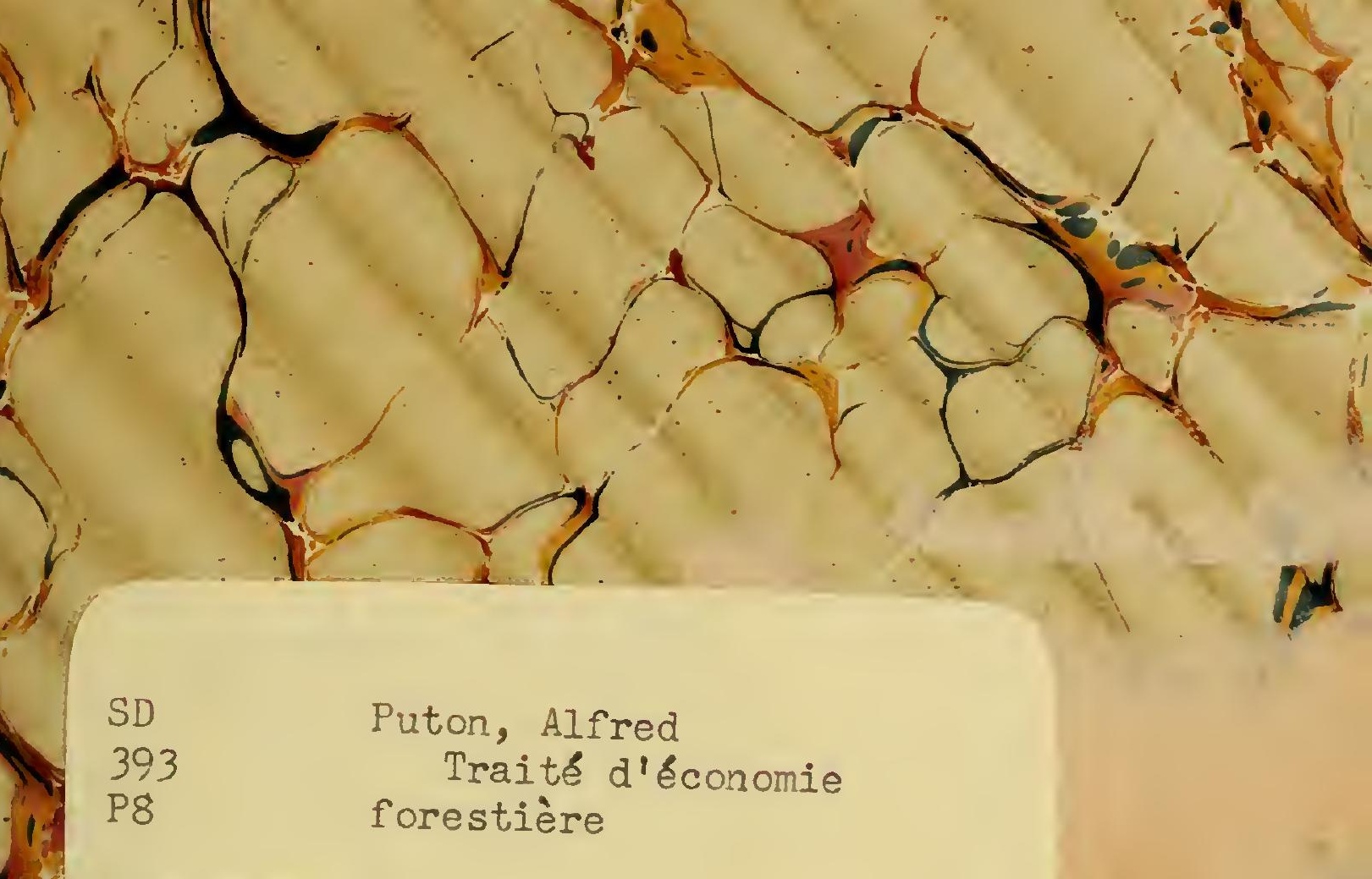

BioMed

UNIVERSITY OF TORONTO LIBRARY.
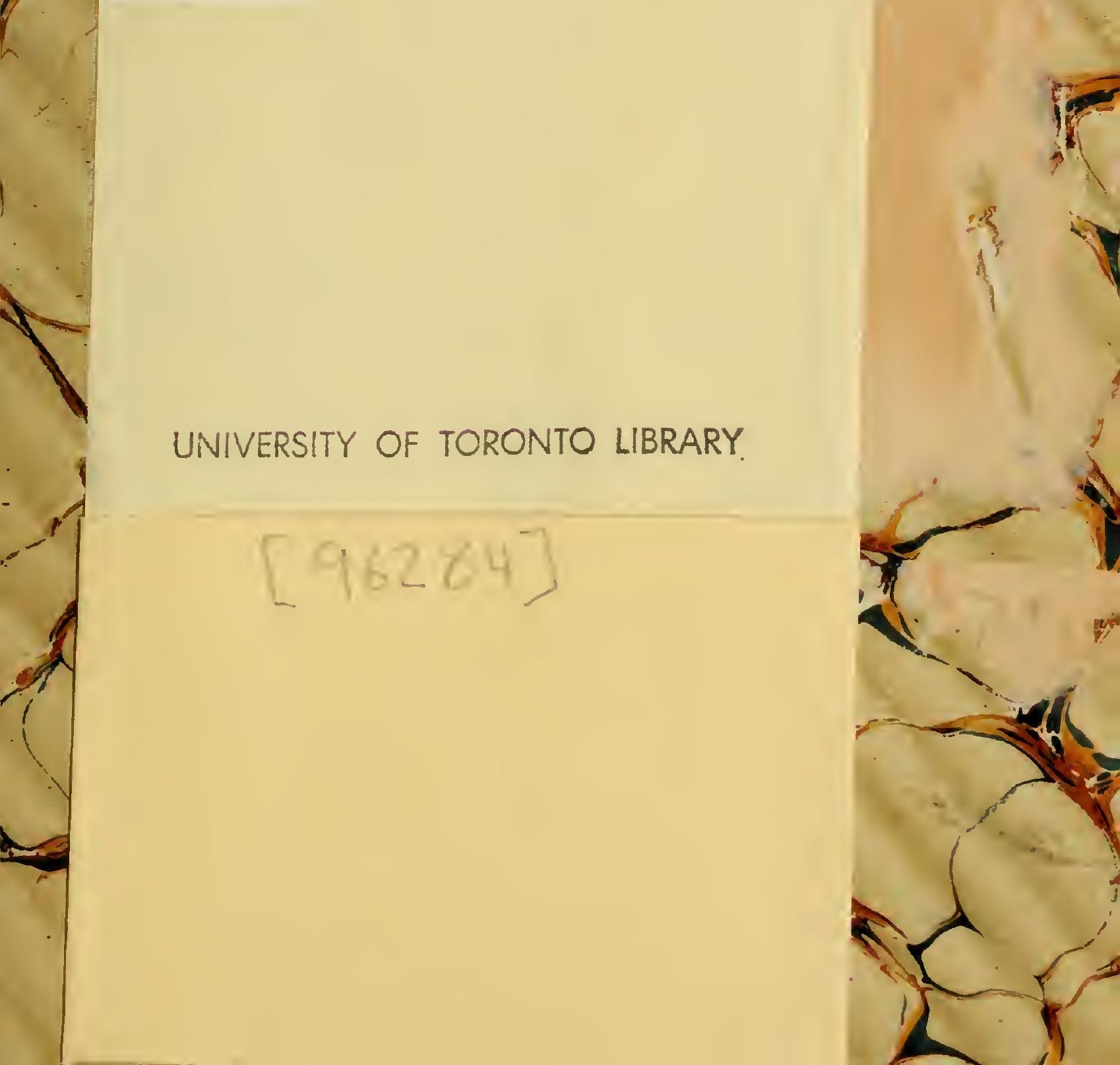
